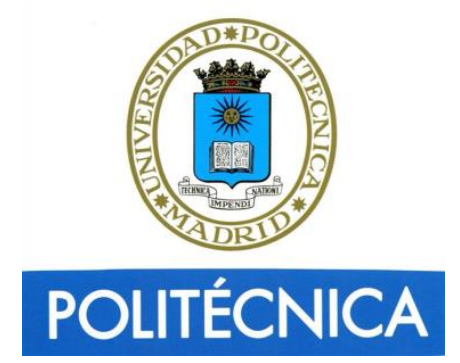

Universidad Politécnica de Madrid

Escuela Técnica Superior De Ingenieros Industriales

\title{
Traffic Conflict Detection and Resolution (CDR) in ERTMS System
}

\section{Taomei Zhu}

Director of the dissertation:

Prof. Dr. José Manuel Mera Sánchez de Pedro

DOCTORAL DISSERTATION

2016 



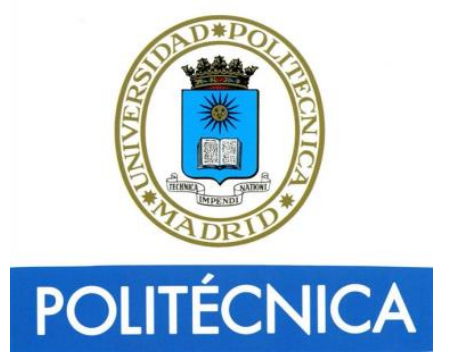

Departamento de Ingeniería Mecánica

\title{
Traffic Conflict Detection and Resolution (CDR) in ERTMS System
}

\author{
Taomei Zhu
}

Director of the dissertation:

Prof. Dr. José Manuel Mera Sánchez de Pedro 


\title{
Detección y Resolución de Conflictos (CDR) de Tráfico en el Sistema ERTMS
}

\author{
Tesis doctoral \\ Universidad Politécnica de Madrid
}

Madrid, 2016

Taomei Zhu

Director: Prof. José Manuel Mera Sánchez de Pedro

Doctor Ingeniero Industrial

Departamento de Ingeniería Mecánica

Escuela Técnica Superior de Ingenieros Industriales

C/ José Gutiérrez Abascal, 2

28006 Madrid 
Tesis Doctoral

\section{Detección y Resolución de Conflictos (CDR) de Tráfico en el Sistema ERTMS}

Tribunal nombrado por Magfco. y Excmo. Sr. Rector de la Universidad

Presidente: Dr. Francisco Javier González Fernández

Secretario: Dr. Santiago Tapia Fernández

Vocal: Dr. José Antonio Jiménez Redondo

Vocal: Dr. Jaime Tamarit Rodríguez

Vocal: Dr. Fernando Montes Ponce de León

Suplente: Dr. Ignacio Jorge Iglesias

Suplente: Dr. Antonio Fernández Cardador 



\section{Preface}

I started my speciality in traffic information and control engineering in 2004, and I have been studying on railway traffic management and related systems for almost eight years, in China and Spain. I am interested in all the advanced technologies and intelligent systems that have driven substantial progresses to railway industry. I have always been dreaming that what an honour and pleasure if I could work out better methods in some aspects and implement them to improve the performance of existing railway systems. Obviously, it is never easy. I really feel lucky that I have not given up efforts and on this way I have gained a lot of help and support from my professors, colleagues and friends. Here, I would like to express my particular gratitude to all these people who appeared in my $\mathrm{PhD}$ study stage.

Thanks to Prof. Angel Alvarez and Dr. Yang Wang, I had the opportunity to know Railway Technology Research Centre (Centro de Investigación en Tecnologías Ferroviarias, CITEF) at Polytechnic University of Madrid (UPM), in 2011. Soon afterwards, I could establish contact with Prof. Jose Manuel Mera who later became my thesis supervisor. In the same year, I joined CITEF and started my study and research work in Spain. I had mainly involved in the research and development of simulation and testing bench in Chinese Traffic Control System Level 3 at State Key Laboratory of Rail Traffic Control and Safety, Beijing Jiaotong University. And at CITEF, I shifted the research focus on to European Railway Traffic Management System (ERTMS) related technologies and applications on which my thesis is based.

Sincerely, I would like to give my very thanks to Prof. Jose Manuel Mera for his patient guidance and discussions during these years, in particular, for his encouragement and trust in my slow research findings during the most difficult time. Thanks to his support, I could access to the resources at CITEF and participate in practical projects. Many times I felt myself an exception in the research centre. Also for his reason, I gradually gained the belongingness at CITEF meanwhile acquiring knowledge and experience.

So many thanks are due to my kind colleagues at CITEF. Miguel provided the interlocking data and application support for my case studies; Eduardo, Jesús and Manuel gave me the guidance in using HAMLET, which were the most important tools I used in these projects and case studies; Javier and María provided the initial research material on ERTMS; Alberto helped me to drive the simulated train; Carlos gave me all kinds of technical support during these years; and the secretaries Mayra, Emi and Carmen helped me with all these daily matters. I must thank to all my colleagues at CITEF for their patience and encouragement in my Spanish study. I have been increasingly enjoying this appealing language abounding with sentiments and expressions. Here I would like to count them down in case that I could not find their names when I get old: Alejandro, Adela, Alberto, David, Emi R., Edu F., Edu C., Felix, 
Ginés, Ignacio, Israel, Ivan, Jesús, José L, Juan D, Luis M., María, Mayra, Mario, Mariano, Manuel, Miguel, Pablo, Pedro, Ramón, etc.

I would also like to give my thanks to the professors of my courses in the first two years at UPM, especially, to Prof. Jesús Juan Ruiz whose classes of statistics inspired me to introduce statistical methodology to railway data analysis; to the professors at INSIA whose classes of vehicle dynamics, biomechanics, accident investigations, transport engineering, etc., extended my knowledge domain and enriched my scientific research methods; and also to Ángel García Beltrán and Santiago Tapia Fernández, the professors of programming, whose classes amplified my horizon in software design and development. I am grateful to all the professors in the department, Antonio, Maria Luisa, Gregorio, Joaquín, Berta, etc., for their concern and help.

A special thank must go to Jesús Misas, who let me urge myself to work hard to make every step forward and feel free to be an "hija china" in Spain.

Also many thanks are for a great many nice friends I met in Madrid, especially, to Yang, Hao, Guanglong, Yu, Wei, Jing, Xu, Lu and Javier who made my first-period life in Madrid much easier, to Yunlai who gave me thoughtful advice in the final stage of my PhD study, to Pan who inspired me to keep doing sports, to Napaporn who gave me so much encouragement to be braver and more optimistic, and to all these international friends I had met in language classes who let me enjoy the multicultural life in Madrid.

Furthermore, heartfelt thanks must go to my family, who unconditionally believed that their little girl would never be wrong, even this time she was leaving for Europe where they had never been. I was quite ashamed, meanwhile, enjoying their limitless respect, confiding and love. To conclude, I still believe that no efforts and faiths will be in vain. ¡Ánimo!

\section{Taomei Zhu}




\section{Abstract}

This thesis addresses the conflict problem in railway traffic. Although the railway traffic management nowadays can partly be carried out by a traffic control system (or a traffic management system), the control system is insufficient to make operational decisions once the occurrence of disturbance gets frequent, particularly at the junctions with dense traffic. Traffic conflicts may arise along with the disturbances. The traffic controllers (and dispatchers) are often required to handle these unexpected conflicts in real-time traffic control. In fact, conflict detection and resolution (CDR) has become one of the most important and difficult tasks in daily railway traffic management. In ERTMS/ETCS, while all advanced technologies are employed to increase the train speed and to reduce the headway interval, increasing challenges of real-time conflict resolution are encountered as well. This thesis focuses on the methodologic realization of conflict detection and resolution and aims to develop a CDR support system for railway traffic control and management.

\section{Modelling}

To describe the environment of the conflict detection and resolution problem, the railway traffic is modelled via its partial models of train, infrastructure and timetable. These partial models are data-based.

- Either an original timetable or an updated (rescheduled) timetable is represented with the purpose of providing common goals for the involved entities in the railway system and organising the related resources in real-time traffic control.

- The train model consists of the relatively static train path and its derivatives and the dynamic train movement information, such as train position, train speed, the occupied track, the operation times and further movement authority, etc.

- The infrastructure model is established both from a line (or a network) range and a region range. The former is described by stations and corridors between stations (that are all named sections). The latter is mainly represented by routes and segments, as well as the structural and restrictive relationships among the local routes and segments. The track description data for dynamic train control is also based on the instantaneous route states and segment states in infrastructure model.

Conflict signs at an earlier stage of potential conflicts are expressed based on the proposed models. 


\section{Conflict detection}

Conflict detection procedure and feasible approaches are studied separately (from conflict resolution approaches). Based on the train model and the infrastructure model, respectively, the approaches of train trajectory tracing and infrastructure state transition are proposed for short period traffic prediction and conflict detection.

- In train trajectory approach, the detailed train movement information is tracked. The correlations of each two dimensions are cooperatively supervised. Particularly, the deviations of distance, track usage, and the speed are detected in the multi-dimensional trajectory profiles.

- The infrastructure state transition approach is based on proper state transition maps and corresponding relation matrices. Historical segment and route states (state vectors) are stored to obtain empirical state transition maps. These maps are applied to the abnormity detection in dynamic traffic environment, and the structural constraints of infrastructure topology and route compatibilities are applied to the prediction of potential conflicting situations.

The conflict detection procedure is concerned with the predictive traffic in a short period of prediction horizon based on the current collected data. The two approaches cooperate to predict the traffic and detect potential conflicts and significant deviations. The detected conflicts or conflict risks are grouped with different identifying code, so that corresponding resolution approaches can be implemented to prevent the conflicts.

\section{Conflict resolution}

According to the categories of the detected conflicts and the early warnings of conflict, two-layer structure of conflict resolution methodology is established.

- One layer is to solve the routing problem in station area continuously when potential route conflicts are predicted. The microscopic times on related segments, train route selection and train order decisions at joint points are considered in this layer.

- On the second layer, the variable scope rescheduling approach is proposed to prevent the propagation of potential conflicts in a wider area. The interstation conflicts, such as headway conflicts and delay propagation, are mainly resolved on this layer. As it is named, the rescheduling scope is decidable according to the specific conflict size.

In this thesis, a set of mixed integer linear programming formulations are expressed for both local routing and variable scope rescheduling. Flexibly, new dispatching strategies in local routing and other excellent algorithms in rescheduling are possible to add to the corresponding layer. 


\section{Agent-based conflict detection and resolution (CDR) support system}

Agent-based modelling approach is applied to the design of conflict detection and resolution support system for railway traffic control and management. The proposed models and approaches for conflict detection and resolution are integrated in the agent model.

The CDR support system (named D-Agent) is modular designed. Six basic modules are included. They are local database, knowledge base, skill base, data processing, reasoning mechanism and communication interfaces. The partial models are functioning in the module of data processing, firstly filtering and reforming the collected data. The approaches of conflict detection and resolution are principally implanted as the kernel skills of the D-Agent. The solutions to potential conflicts are communicated via the DAgent interfaces.

\section{Conflict detection and resolution in ERTMS system}

In the applications of ERTMS/ETCS system, the inevitability of the influence on CDR functions that is caused by different ERTMS application levels and the necessity of adapting the CDR to different ERTMS application levels are discussed. The final conclusion is that a level adapter function can be added to the agent-based CDR support system for the ERTMS system, in order to allow the appropriate CDR approaches to be applied in a specific ERTMS/ETCS level or in the situations where the ERTMS/ETCS level transfers. 


\section{Resume}

Esta tesis se dirige a la problemática de conflictos dentro del tráfico ferroviario. Aunque, en nuestros días, la gestión del tráfico ferroviario puede ser parcialmente llevada a cabo por un sistema de control de tráfico (o un sistema de gestión de tráfico), dicho sistema de control es insuficiente para tomar decisiones operacionales una vez que la ocurrencia de perturbaciones se vuelve frecuente, particularmente en los puntos de unión con tráfico significativo.

El personal de control de tráfico es a menudo requerido para manejar estos conflictos inesperados dentro del control en tiempo real del tráfico. De hecho, la detección y resolución de conflictos (CDR) ha llegado a ser una de las más importantes y difíciles tareas en el día a día de la gestión del tráfico ferroviario. En ERTMS/ETCS, mientras todos los avances tecnológicos son empleados en incrementar la velocidad del tren y reducir el intervalo entre trenes, los desafíos de la resolución de conflictos en tiempo real, que son encontrados, se incrementan también. Esta tesis se centra en la realización de métodos para la detección y resolución de conflictos con el objetivo de desarrollar un sistema CDR de apoyo para el control y gestión del tráfico ferroviario.

\section{Modelaje}

Para describir el entorno del problema de la detección y resolución de conflictos, el tráfico ferroviario es modelado vía sus modelos parciales de la infraestructura, el material rodante (tren) y los horarios programados. Estos modelos parciales están basados en datos.

- Un horario programado original o actualizado (reprogramado) es representado con el propósito de proporcionar unos objetivos comunes para las entidades involucradas en el sistema ferroviario y organizar los recursos relaciones en el control de tráfico en tiempo real.

- El modelo del tren consiste de las rutas relativas de los trenes y sus derivadas junto con la información dinámica del movimiento de los trenes, tales como la posición y la velocidad de los trenes, los circuitos de vías ocupados, los tiempos de operación, las autoridades de movimiento, etc.

- El modelo de la infraestructura es establecido desde un rango de línea (o una red) y un rango regional. El primero es descrito por las estaciones y los corredores entre las estaciones las cuales son nombradas secciones. Y el último es principalmente representado por rutas y segmentos. Los datos de descripción de las vías para el control dinámico del tren es también basado en los estados instantáneos de las rutas y segmentos en el modelo de la infraestructura.

Los signos de conflictos en las fases tempranas de los potenciales conflictos son expresados mediante los modelos propuestos. 


\section{La detección de conflictos (CD)}

El procedimiento de detección de conflictos y las aproximaciones factibles son estudiados separadamente (desde aproximaciones de resoluciones de conflictos). Basados en el modelo del tren y de la infraestructura, respectivamente, las aproximaciones de las trayectorias seguidas por los trenes y la transición de estados de la infraestructura son propuestas para predecir el tráfico en cortos periodos y detectar conflictos.

- En las aproximaciones de las trayectorias de los trenes, la información detallada del movimiento de los mismos es monitorizada. Las correlaciones de cada una de las dos dimensiones son supervisadas cooperativamente. Particularmente, las desviaciones de la distancia, las vías empleadas, y las velocidades son detectadas en los perfiles de trayectorias multidimensionales.

- La aproximación de las transiciones de estado de la infraestructura está basada en los precisos mapas de transición de estado y las correspondientes matrices de relación. Los históricos de segmentos y los estados de las rutas (vectores de estados) son almacenados para obtener mapas de transición empíricos. Estos mapas son aplicados a la detección de anormalidades en el entorno dinámico ferroviario, y a su vez, las restricciones estructurales topológicas de la infraestructura y las incompatibilidades entre rutas son aplicadas a la predicción de potenciales situaciones de conflicto.

El procedimiento de detección de conflictos se preocupa de la predicción del tráfico en cortos periodos del horizonte de predicción basado en los datos actuales recogidos. Las dos aproximaciones cooperan para predecir el tráfico y detectar los potenciales conflictos y desviaciones significativas. Los conflictos detectados o los riesgos de los mismos son agrupados según diferentes códigos identificativos, por eso las correspondientes aproximaciones para su resolución pueden ser implementadas para prevenirlos.

\section{La resolución de conflictos $(\mathrm{CR})$}

Según las categorías de los conflictos detectados y los tempranos avisos de conflicto, una metodología de resolución de los conflictos estructurada en 2 capas es establecida:

- Una capa se encuentra resolviendo continuamente el problema de establecimiento de rutas dentro del área de estación, cuando potenciales conflictos entre rutas son predichos. Los tiempos sobre los segmentos involucrados, la selección de las rutas y las decisiones de órdenes en los puntos de conexión son considerados en esta capa.

- En la segunda capa, una aproximación al nivel de reprogramación de los horarios de los trenes es propuesta para prevenir la propagación de potenciales conflictos dentro de una amplía área. Los conflictos entre estaciones, tales como conflictos de alcance de trenes y propagación de retrasos, son 
principalmente resueltos dentro de esta capa. Como es mencionado, el ámbito de la reprogramación de los horarios es determinado según el tamaño del conflicto especifico.

En esta tesis, un conjunto de fórmulas basadas en la programación "mixed integer linear" son expresadas para el establecimiento de rutas localmente y la reprogramación de horarios de ámbito variable. Flexiblemente, es posible añadir nuevas estrategias para el establecimiento de rutas localmente así como nuevos mejorados algoritmos en la reprogramación de horarios, únicamente modificando la correspondiente capa.

\section{Sistema de apoyo para la detección y resolución de conflictos basado en Agente}

Una aproximación de modelaje basada en agente es aplicada al diseño de un sistema de apoyo en la detección y resolución de conflictos para el control y gestión del tráfico ferroviario. Los modelos y aproximaciones propuestas para la detección y resolución de conflictos están integrados dentro del modelo del agente.

El sistema de apoyo CDR (nombrado D-Agent) es de diseño modular. Está formado por 6 módulos básicos, que son respectivamente una base de datos local, una base de conocimiento, una base de habilidad, un procesamiento de datos, un mecanismo de razonamiento, y unas interfaces de comunicación. Los modelos parciales se encuentran funcionando dentro del módulo de procesamiento de los datos, primeramente filtrando y reformando los datos recogidos. Las aproximaciones de detección y resolución de conflictos están implantadas principalmente como las habilidades fundamentales del DAgent. Las soluciones para las potenciales situaciones de conflicto son comunicadas vía las interfaces del D-Agent.

\section{Detección y resolución de conflictos en el sistema ERTMS}

En las aplicaciones dentro del sistema ERTMS/ETCS, la previsibilidad de la influencia sobre las funciones del CDR debidas a los diferentes niveles operacionales de ERTMS y la necesidad de adaptar el CDR a dichos diferentes niveles son discutidas. La conclusión es que una función de adaptación al nivel operacional del ERTMS puede ser añadida al sistema de apoyo CDR para el sistema ERTMS, que permita aproximaciones del CDR apropiadas para ser aplicadas en un especifico nivel ERTMS/ETCS o en las situaciones donde el nivel ERTMS/ETCS es modificado. 


\section{Contents}

PREFACE

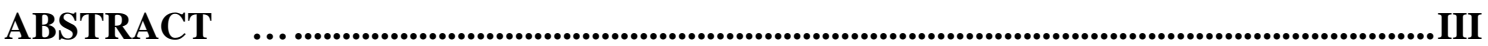

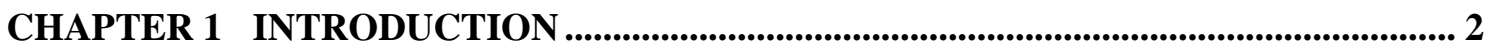

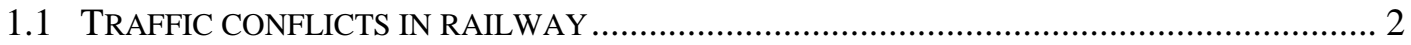

1.1.1 Hierarchical characteristic of railway conflicts ................................................. 2

1.1.2 Conflict detection and resolution in ERTMS system ......................................... 4

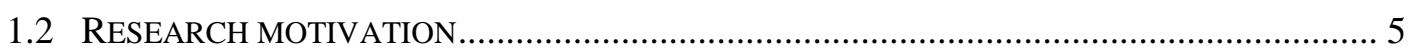

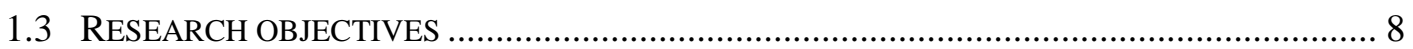

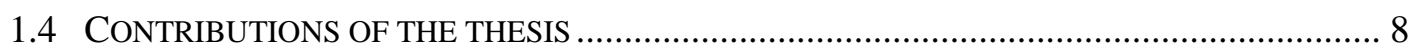

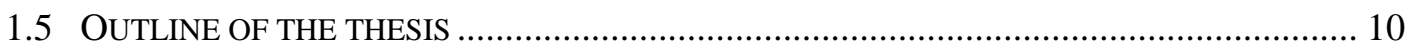

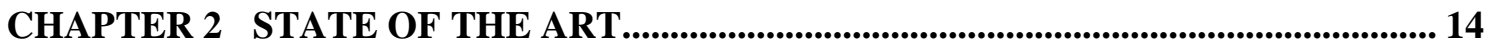

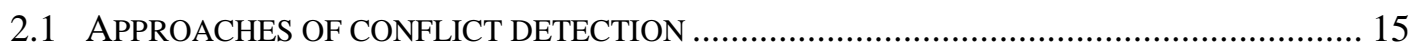

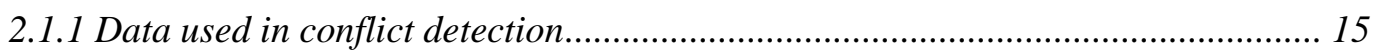

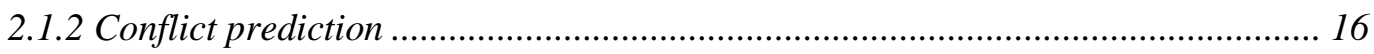

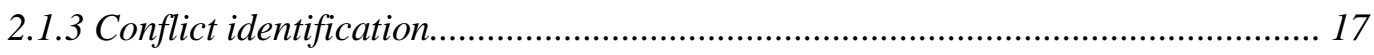

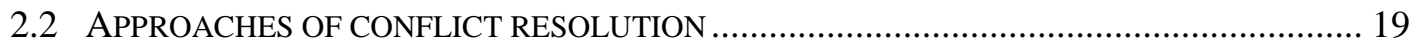

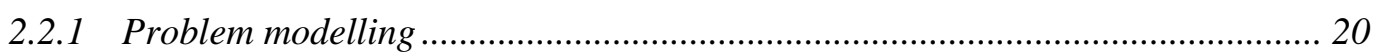

2.2.2 Algorithms on conflict resolution .................................................................. 23

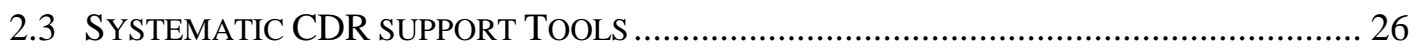

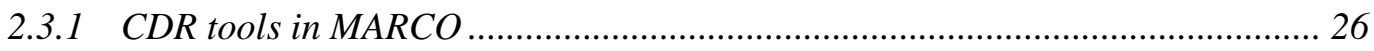

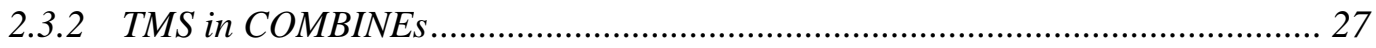

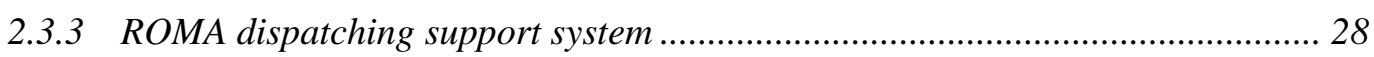

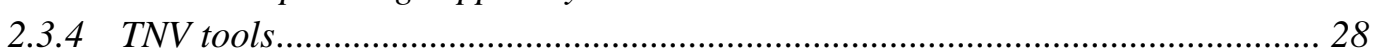

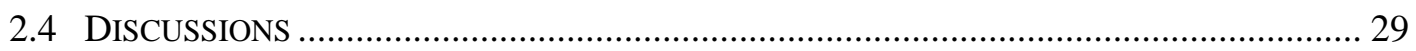

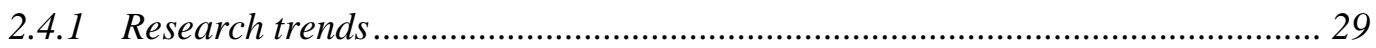

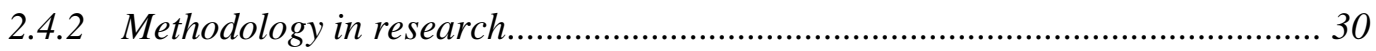

CHAPTER 3 RAILWAY TRAFFIC MODELLING ............................................................ 34

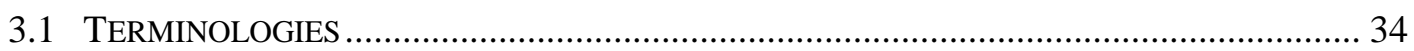

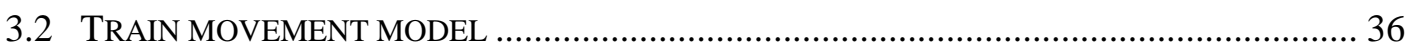

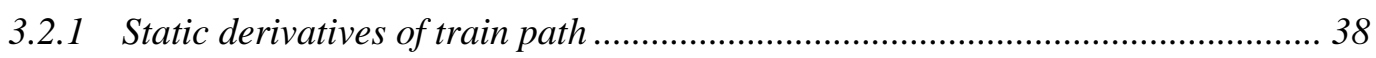

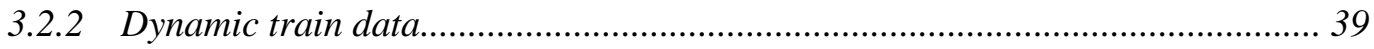

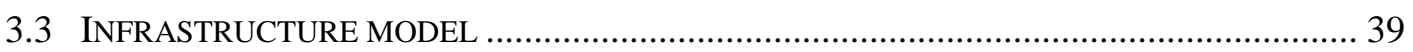

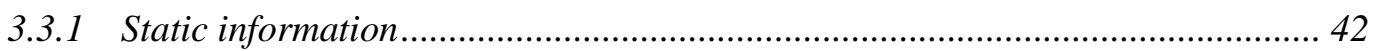

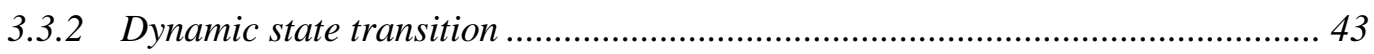

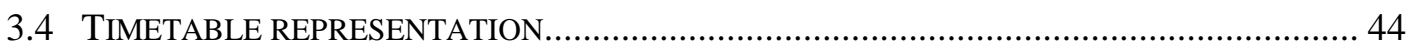

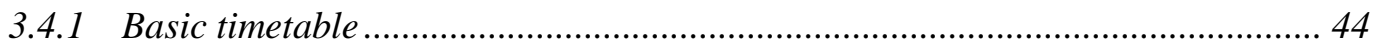

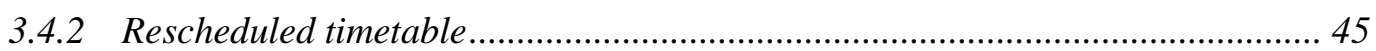

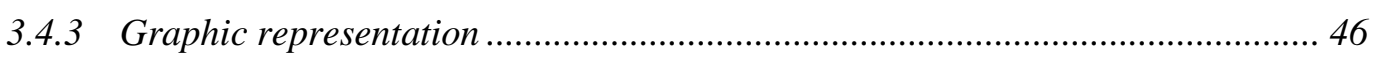

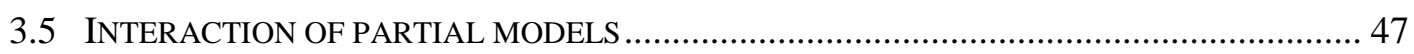

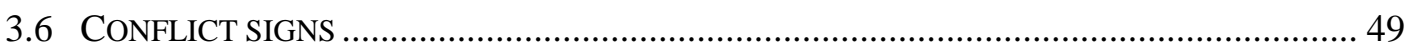

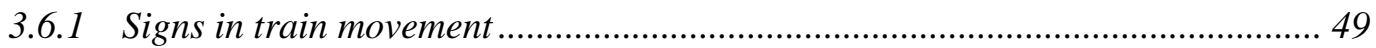




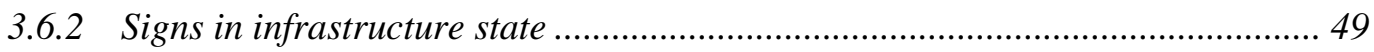

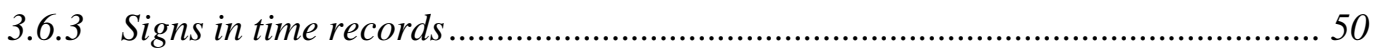

CHAPTER 4 CONFLICT DETECTION ...........................................................................52

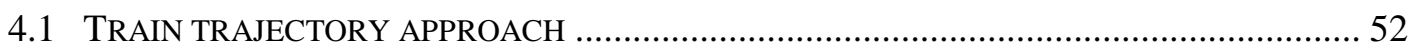

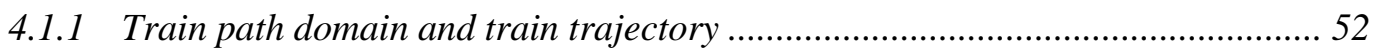

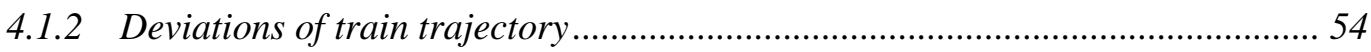

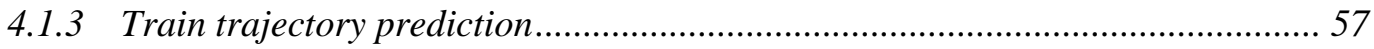

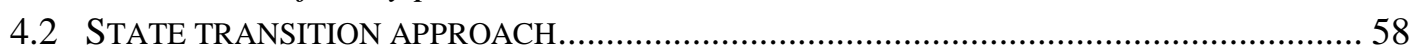

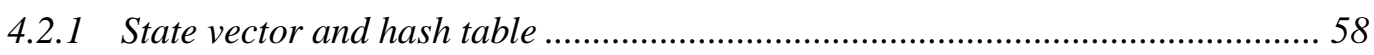

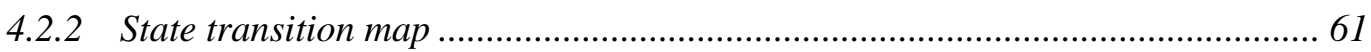

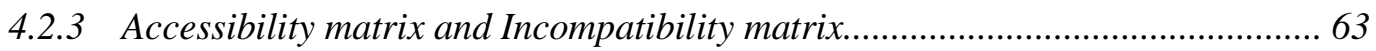

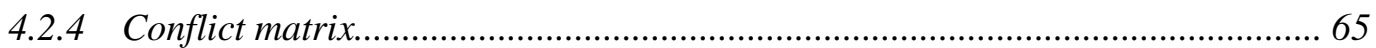

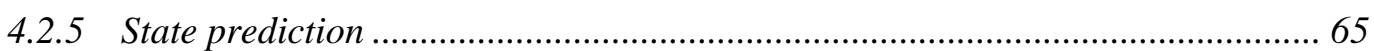

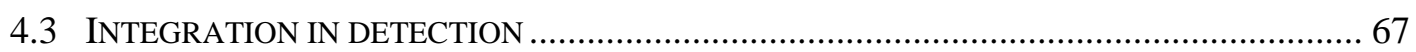

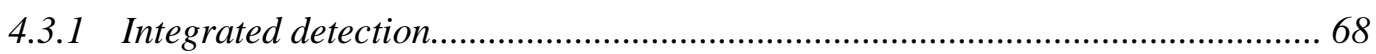

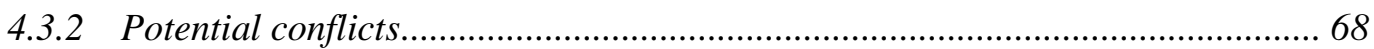

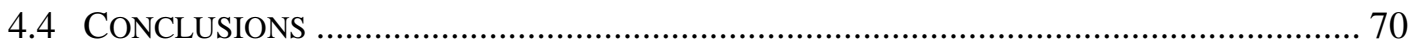

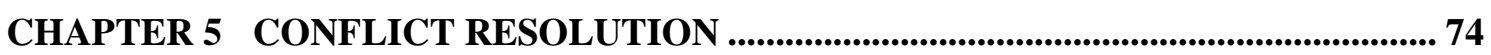

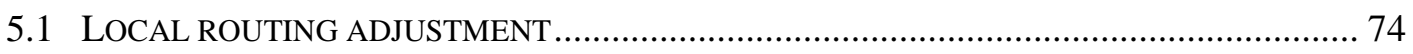

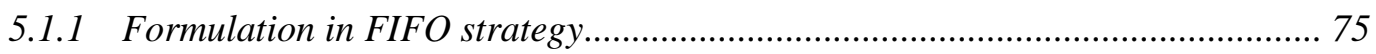

5.1.2 Formulation with changeable train order .......................................................... 77

5.1.3 Formulation with train route changeable ......................................................... 78

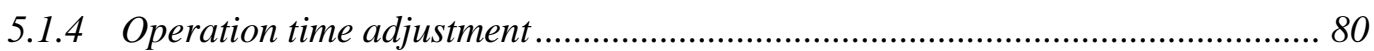

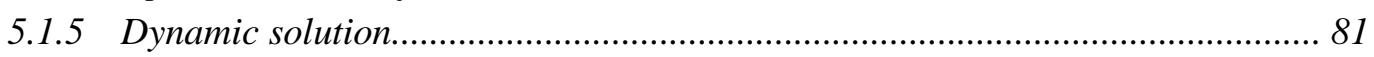

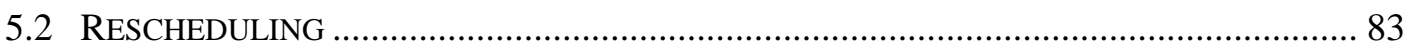

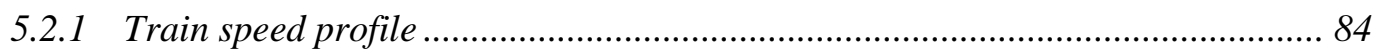

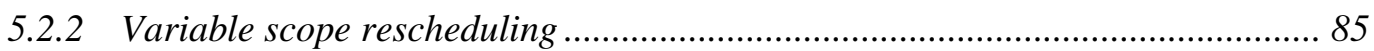

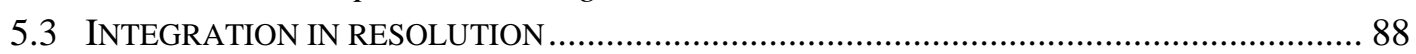

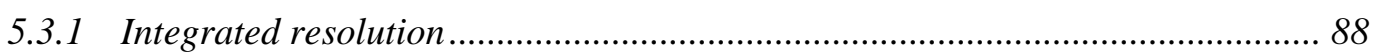

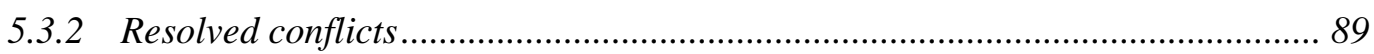

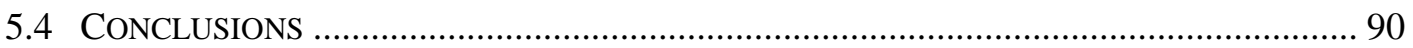

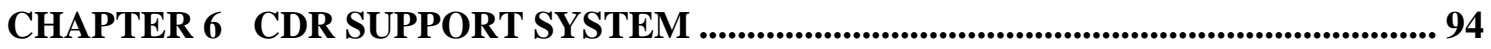

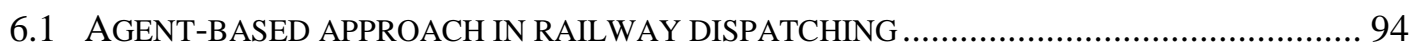

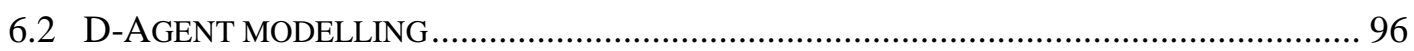

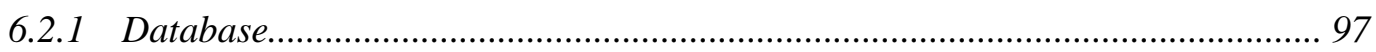

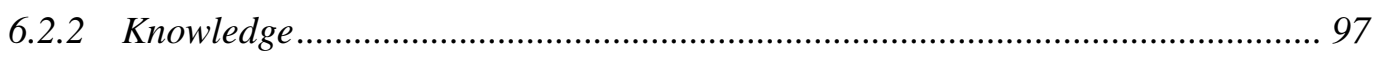

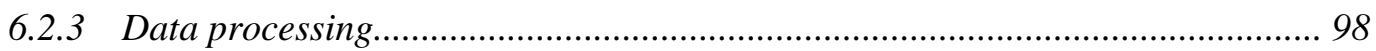

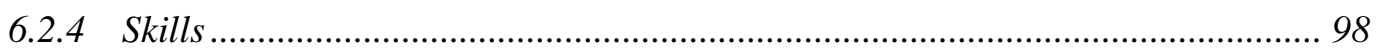

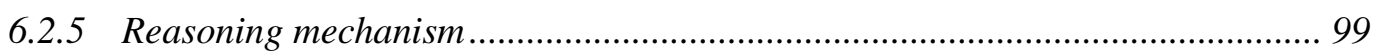

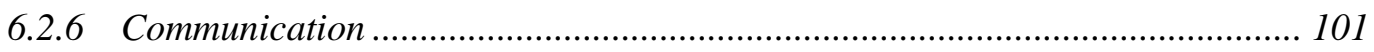

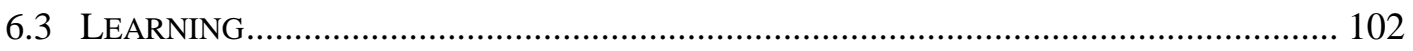

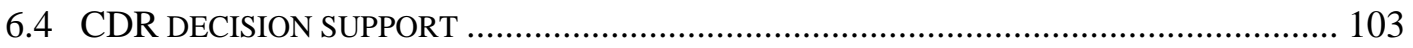

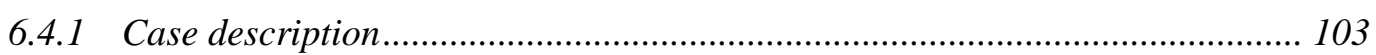

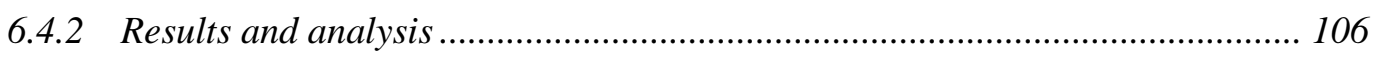




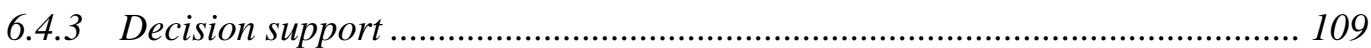

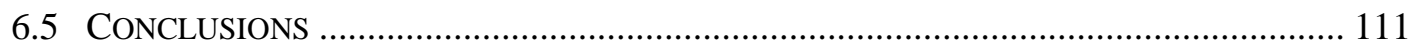

CHAPTER 7 CDR IN DIFFERENT ERTMS/ETCS APPLICATION LEVELS............ 114

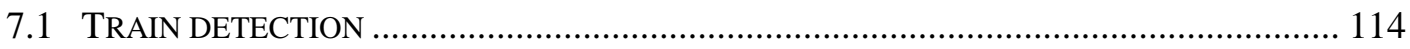

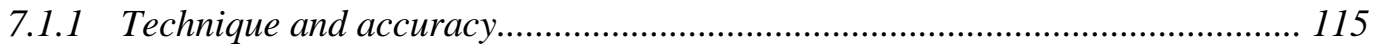

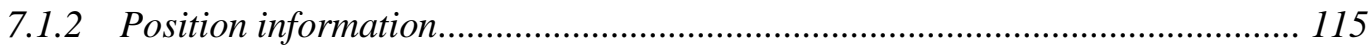

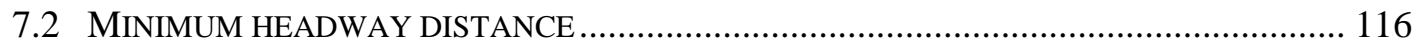

7.2.1 Headway under fixed block principles ............................................................. 116

7.2.2 Headway under moving block principles ....................................................... 117

7.2.3 Factors of minimum headway control.......................................................... 119

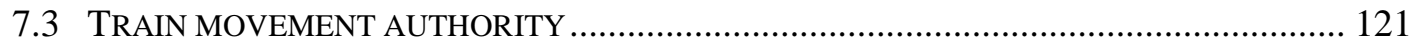

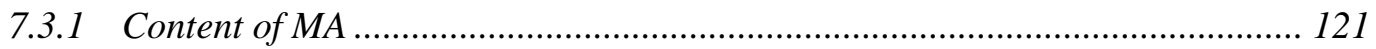

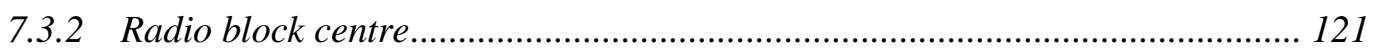

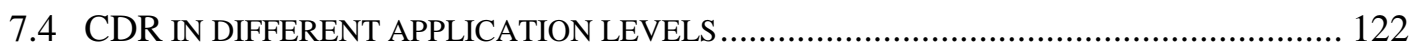

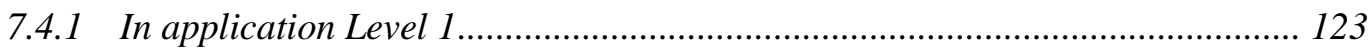

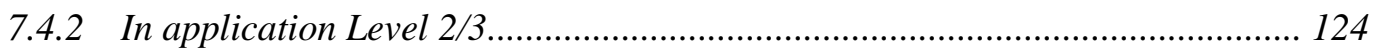

7.5 ERTMS/ETCS LEVEL ADAPTER IN CDR SUPPORT SYSTEM...................................... 126

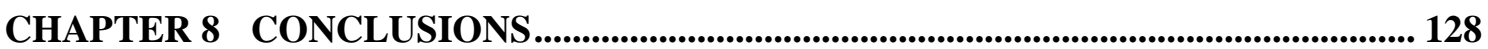

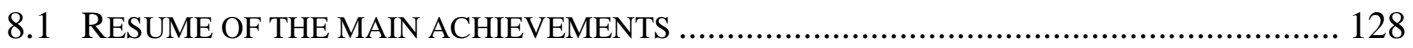

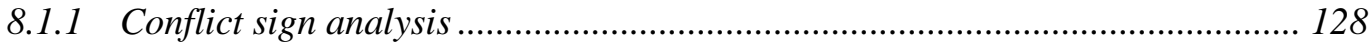

8.1.2 Conflict detection and resolution approaches............................................... 129

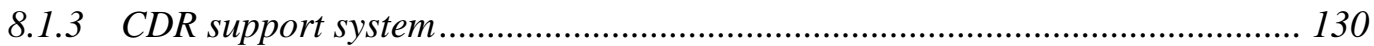

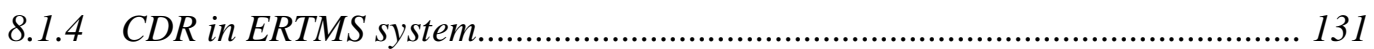

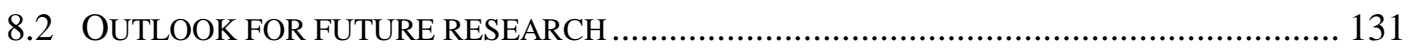

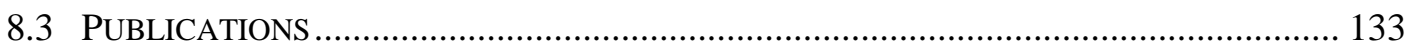

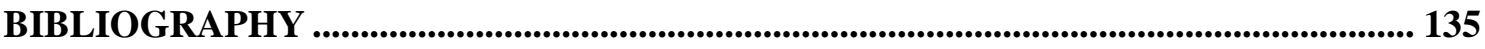

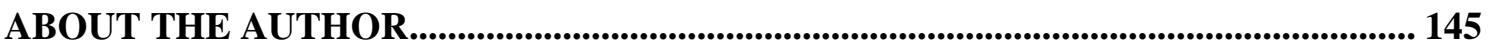





\section{Chapter 1 Introduction}

The scope, the motivation, the objectives and the contributions of the work are introduced. 


\section{Chapter 1 Introduction}

\subsection{Traffic conflicts in railway}

In spite of the technological development in the whole railway industry, traffic conflicts are still unavoidable in daily railway operation and in fact detecting and resolving conflicts takes a very great part of dispatching time, especially in complexly networked railway systems.

\subsubsection{Hierarchical characteristic of railway conflicts}

A classical hierarchical approach of classifying the management activities of railway system as three levels (strategic, tactical and operational) was firstly proposed in economics area by (Anthony, 1965). It started to be well adopted in railway planning process after the citation and application in (Bussieck et al., 1997). Figure 1-1 shows a hierarchical graph of railway traffic management, which has ever been described in some previous work (Assad, 1980; Borndörfer et al., 2011; Caimi, 2009; Lindner, 2000; Lusby et al., 2011a; Törnquist, 2005).

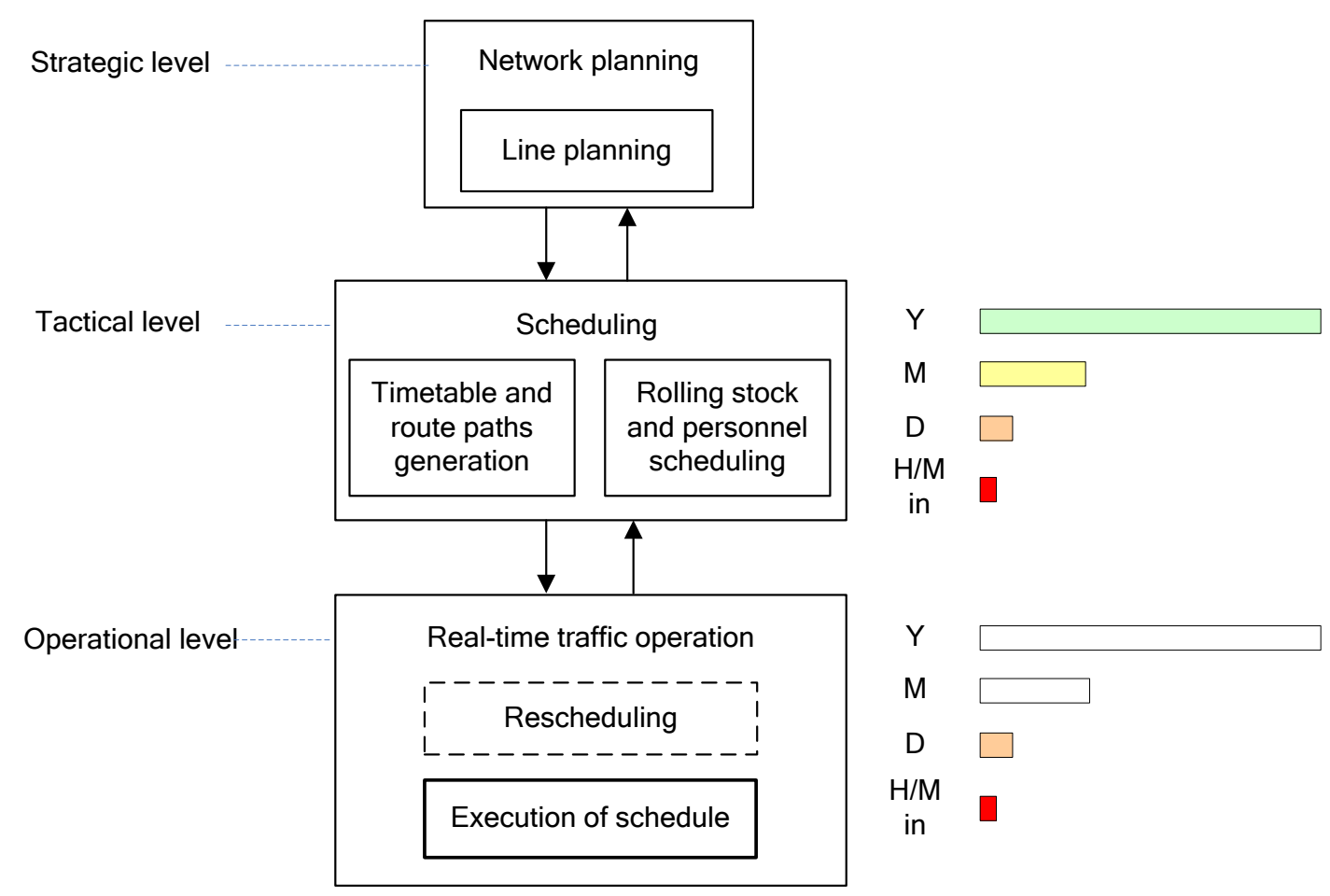

Figure 1-1 The hierarchical railway traffic process

At strategical level, feasible railway lines and the transport network are decided and designed. On this foundation schedules are generated at tactical level comprehensively considering the capacity requirement, technical aspects of vehicles and infrastructure and other resources. One best-known term of schedule is the public timetable of passenger trains, which usually could be valid in the long term yet it has included 
detailed information to minutes. The routine actions in everyday railway traffic control and management are in scope of operational level that may not always be planned actually.

Conflicts may exist at all levels, varying in accordance to the corresponding requirement at each level, e.g. major conflicts at strategic level may be the ones between the public transport policy and possible infrastructure investments (Hooghiemstra, 1996), yet which are not considered in this thesis; the required services to the passengers conflicting with the benefits of railway companies, conflicts in rolling stock and personnel management are not included either, although they are important factors to generate and optimise the basic scheduling; what we focus on are those real-time conflicts occurring in daily traffic operation, e.g. train delay propagation, route deadlock, etc. which are usually called dispatching conflicts; some influential algorithms and methods applied in scheduling stage (at tactical level) are investigated to collect as many approaches as possible that may be suggestive to resolve the dispatching conflicts, where conflict free is one of the primary objectives of off-line timetable and route planning.

Restricted to the operational level, problems of traffic conflicts at this level are subsequently devised into conflict detection (CD) and conflict resolution (CR). As a prerequisite of conflict detection and resolution, railway conflicts should be defined first of all. Although in a lot of previous literature it was either assumed that conflicts had been definite or detected so that only the CR problems were focused on, or considered delays and disturbances were the starting points of their work, definitions of railway conflicts have been made in quite a few references. In general, conflicts are described in sharing the spatial resources (such as block section, route, headway, etc.), e.g. Goverde et al. (2008) defined that a route conflict occurs when the movement authority of a train is restricted by a conflicting preceding train. In practice, this implies that a train meets a restricted signal and must prepare to stop before a stop signal protecting an occupied block section. More directly, Corman et al. indicated a potential conflict occurs when two or more trains contend for the same block section simultaneously, requiring a decision on the train order (Corman et al., 2011; Corman et al., 2012a; Corman et al., 2012b). Train conflicts are also defined in terms of headway, i.e. a train conflict is a situation in which two or more trains claim resources in an infeasible manner, namely, in the way that violates a safety regulation, and in most practical cases, conflicts arise when a set of trains fail to respect the safe headway times or the minimum distance headway due to delays (D'Ariano et al., 2007b; Min et al., 2011; Samà et al., 2016a). In a more common sense, a railway conflict is defined as a situation where the scheduled train operation is unachievable because of external problems (Kim et al., 2011). Besides, (Medeossi et al., 2011) proposed a new definition of traffic conflicts that conflicts could be determined by a function of process-time variability, in terms of probability.

In this thesis, we define that a conflict at operational level is a situation in which a train cannot exactly realise its plan due to a disturbance or interruption in the railway 
network. It can either be a conflict between multiple trains or a conflict between train and infrastructure. Here a disturbance is a relatively small perturbation of the railway system that can be handled by modifying the timetable, but without modifying the duties for rolling stock and crew, while an interruption (disruption) is relatively a large incident, requiring both the timetable and the duties for rolling stock and crew to be modified (Cacchiani et al., 2014).

No matter in which form the conflicts are defined, as the names imply, conflict detection (CD) can be considered as the process of identifying when and where the potential conflicts occur, and conflict resolution (CR) then involves determining what and how the appropriate actions should be taken to prevent the conflicts, such as speed adjustment, train re-ordering, rescheduling, etc.

\subsubsection{Conflict detection and resolution in ERTMS system}

To increase capacity on a line or network, applying (or upgrading the existing system to) ERTMS seems the most feasible, effective and economic solution for rail operators in Europe. However, the time span allowing us to find a solution in certain conflicting situation may become shorter for the stricter restriction in train headway and the limited capacity of the line or network (ERA et al., 2014).

It is normally mentioned that the two main components of the ERTMS system are the European Train Control System (ETCS), a standard for on-board train control, and the GSM mobile communications standard for railway operations (GSM-R). Besides, a third basic element of ERTMS system is the European Traffic Management Layer (ETML), the operation management level to optimise train movements by the intelligent interpretation of timetable and train running data.

As Figure 1-2 shows, a conflict detection and resolution (CDR) support system can be embedded in the ETML (or Traffic Management System, TMS) of ERTMS system, sharing the data of both local and global database. Furthermore, conflict detection and resolution functions can be encapsulated to realise as a part of Centralized Traffic Control system (CTC), Traffic Control Centre (TCC) or Operation Control Centre (OCC), which varies in different countries and companies. CDR supplements the functions of ETML (TMS) without adding complexity to the other components of ERTMS system.

Depending on the application level of ERTMS/ETCS, the trackside and on-board subsystems and the communication system used in field could be differently equipped, e.g. the GSM-R radio communication network is used for the bi-directional exchange of messages between on-board subsystems and RBC or radio infill units (ERTMS/ETCS Level 3 or Level 2), but is not developed in the system of ERTMS/ETCS Level 1. Dynamic data of trains and infrastructure can be collected in different ways. It will be discussed in this thesis. 


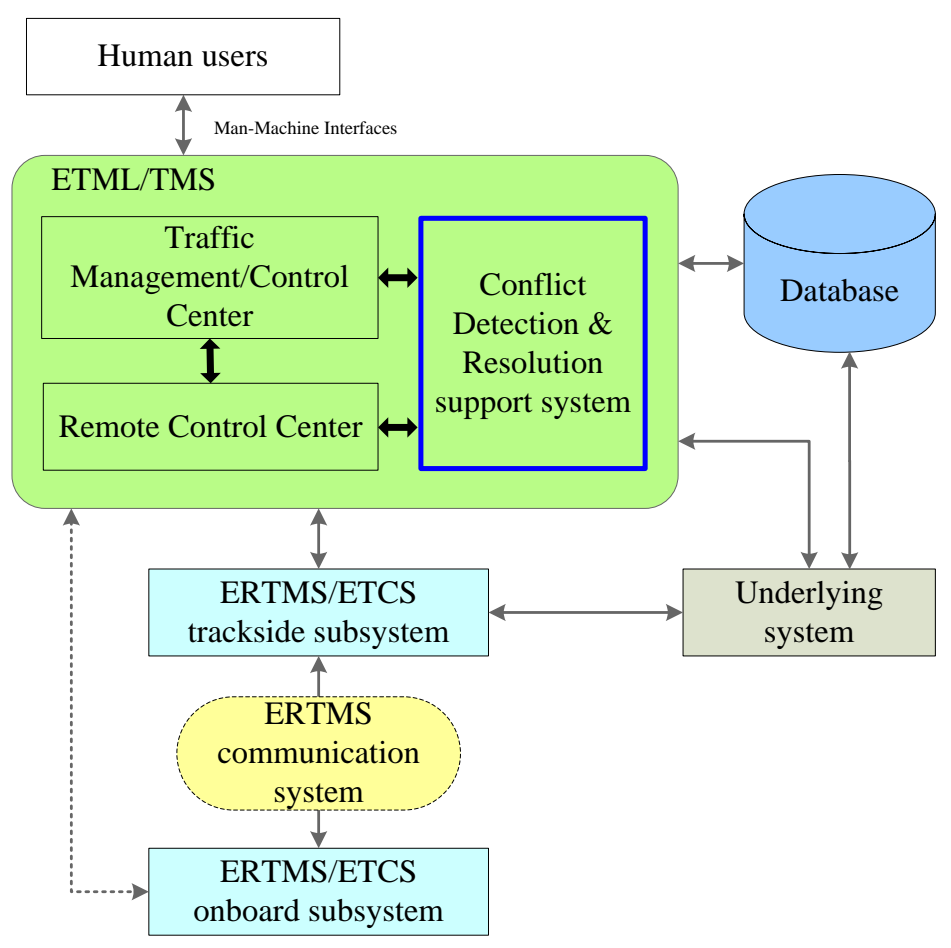

Figure 1-2 Common environment of conflicts detection and resolution in ERTMS system

\subsection{Research motivation}

Although it has been overall developed in railway, the unexpected traffic conflicts usually are still handled as the most complicated tasks by traffic dispatchers. Automatic operation of conflict detection and resolution is in great request. But it is not an easy job indeed and many obstacles are to be overcome. Thus, to investigate the concrete difficulties and probably to handle some of them is the primary motive of this dissertation.

\section{Traffic dispatching difficulties}

Routine work of railway station nowadays can be partly controlled by train dispatching control system or centralized traffic control system, including infrastructure and train states monitoring in the scope of station and its boundaries with neighbour stations, regular train dispatching and route control in station, etc. However, these control systems are not sufficient when the station size and traffic density are increasing, especially when it is accompanied by train delays and other unexpected disturbances. Therefore the dispatcher of station has to deal with all local traffic information and to make quick decisions of rescheduling and rerouting within a very short period of time. To sum up, the main difficulties in railway traffic dispatching include:

- Real-time information collection;

- Communication with multiple entities at the same time;

- Rapid decision-making;

- Highly restricted by safety requirements and operation rules; 
- Meanwhile considering the objectives of economic saving and passenger satisfaction, etc.

In this sense, to develop such support systems with both the rapid calculating advantage of computer and the intelligence of human dispatcher is becoming a quietly urgent target for the investigators, while the other side the train control technology is rapidly developed and train speed record is continually updated.

\section{ERTMS/ETCS applications}

In respect of the ERTMS system, the study of conflict detection and resolution still has a significant sense to substantiate the optimisation of European Traffic Management Layer.

So far, promising progress has been made in ETCS and communication technologies both in the research and in practical applications, but relatively fewer breakthroughs have been done in the ETML or TMS. The cask theory tells that any short board may limit the ERTMS/ETCS system performance. The more advanced in traffic control technology, the more requisite in developing the corresponding traffic management system. Conflict detection and resolution is the most challenging part of daily traffic control and management work. Besides, with the targets of increasing speed and shortening the operation intervals of trains in the ERTMS system, the challenges of traffic management increase correspondingly.

However, quite few CDR systems have systematically described the methodologies of conflict detection and resolution particularly concerning the different application levels of ERTMS/ETCS. It is also interesting to investigate into the factors that may actually influence the realisation of conflict detection and resolution in different ERTMS/ETCS levels.

\section{System control and optimisation}

From a perspective of system control, if (a) and (b) in Figure 1-3 summarised the most common closed-loop system control chart with two-layer feedbacks in railway operations. The inner layer represents that the monitoring system continuously collects data of train movements and infrastructure states and local dispatchers manually adjust the disturbance when it occurs. The second layer undertaking the medium and short term scheduling/rescheduling works when the inner adjustment is ineffective or regional new schedule is requisite. We are still curious about the effects if the CDR modules relevant adjustment are respectively embedded to each layer of the existing system, see (c) in Figure 1-3. Besides, a third layer feedback (that could be off-line) is possible to provide to long term scheduling centre for further timetable optimisation. 


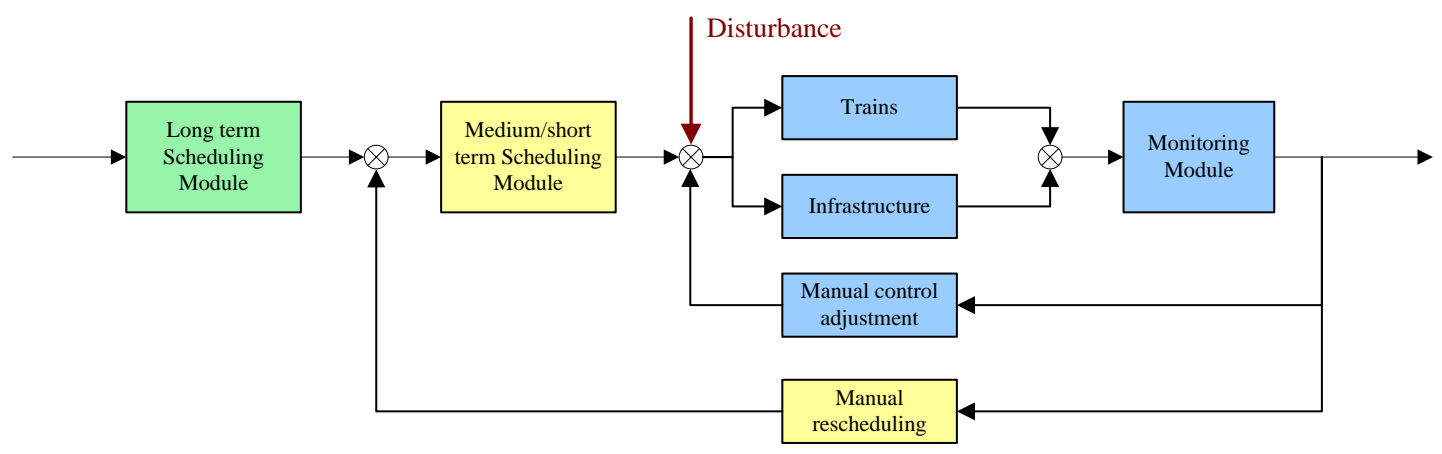

(a) Disturbance handling by manual adjustment and rescheduling

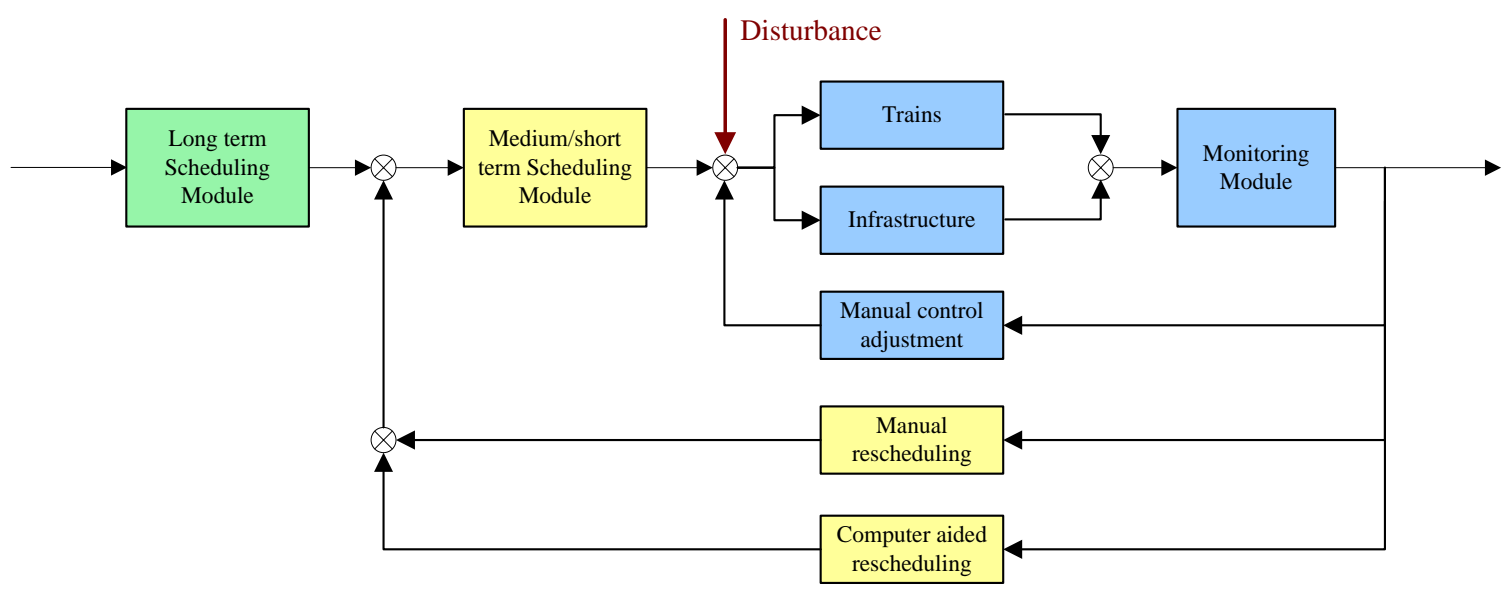

(b) Disturbance handling by manual adjustment and computer-aided rescheduling

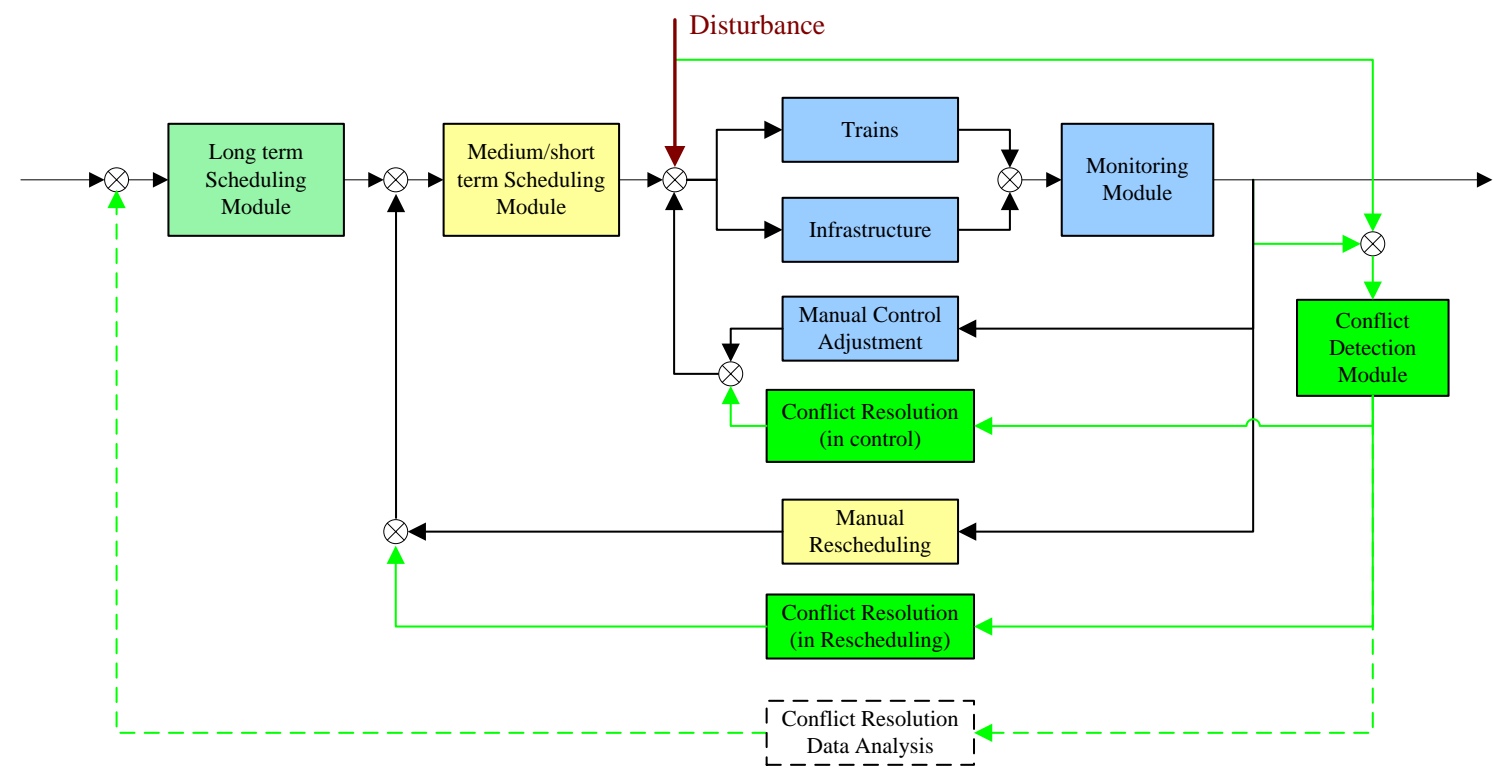

(c) Disturbance handling by embedding conflict detection and resolution modules

Figure 1-3 Railway control and management cycles in different system control patterns 


\subsection{Research objectives}

The ultimate goal of the research on railway traffic conflict is to prevent potential conflicts from happening in practical railway traffic with specific dispatching strategies, e.g. minimum disturbances or total deviation from the published timetable, minimum number of influenced trains, etc.

An applicable objective of the research project is to build an automated conflict detection and resolution system parallel supporting the railway traffic control and management system. As it shows in Figure 1-3 (c), three-layers are in consideration.

- Efficiently detect the disturbance and identify conflicts in a short period of time, and make a direct real-time adjustment to regain a new balanced status of the infrastructure and trains (the inner and fundamental loop);

- Predict according to the current information and properly give short or medium term rescheduling advice to the rescheduling module (the middle loop);

- Provide history data, including conflict records, for long term system performance analysis and timetable optimisation (the outer and global loop).

In order to realise the functions above, approaches in railway traffic prediction, algorithms in rescheduling and dispatching adjustment will be studied and systematic methodology of conflict detection and resolution will be developed.

For the assistance purpose, or liberating the human dispatcher from the traffic dispatching work, the intelligence of CDR support system will be taken into account and appropriate artificial intelligence techniques will be integrated if needed.

From an application perspective, the performance of the proposed conflict detection and resolution system in different ERTMS/ETCS levels has to be discussed as well. Distinctive adaption of CDR to corresponding ERTMS/ETCS levels has to be applied if necessary.

\subsection{Contributions of the thesis}

The main contributions of this dissertation are as follows:

Models of train and infrastructure, under the organization of the timetable, are established to describe the represent the railway traffic. The model of train movement is not based on the kinetics theory of the train, and the model of infrastructure is not designed as interlocking. The models are data-based and they compose the environment of the conflict detection and resolution problem. Conflict signs at an earlier stage of potential conflicts are described based on the proposed models.

In this dissertation, the procedures of conflict detection and conflict resolution are firstly investigated, separately. 
Train trajectory tracing and infrastructure state transition approaches are proposed for short period traffic prediction and conflict detection. These approaches fill the gap of conflict detection methodology that has not been much presented in other related work on conflict detection and resolution problem.

- The detailed train movement information such as train position, train speed, the occupied track and the operation times is tracked in train trajectory approach. Not only the values in each single dimension, but the correlations of each two dimensions are cooperatively supervised. Particularly, the deviations of distance, track usage, and the speed are detected in the multi-dimensional trajectory profiles.

- Then in a novel way, state transition maps are used to describe the railway traffic and its restrictions, taking into account the railway network topology and the route compatibilities. Based on a common state transition map, upcoming traffic states are predicted. Particularly, irregular states are analysed with the relation matrices for both current situation and a short period of future time. Consequently, potential traffic conflicts are identified with detailed conflicting information.

- Potential conflicts and significant deviations (at conflict risks) are coded, with numerical threshold control and formulaic expressions.

Two-layer structure of conflict resolution methodology is established, where a set of mixed integer linear programming formulations are described to find the optimal solution in estimated conflicting situations.

- First layer is to solve the routing problems in station area when potential route conflicts are detected where the microscopic times (at segment level), train route selection and train orders at joint points are considered.

- The second layer is to prevent potential conflict propagation in a wider area via variable scope rescheduling approach, where the rescheduling scope can be decidable according to the conflict size.

- The combination makes the methodology more adaptive to different railway systems. Besides, new dispatching strategies in local routing and wellperforming algorithms in rescheduling are flexible to be added into corresponding layer.

Agent-based modelling approach is applied to design the conflict detection and resolution support system in dynamic dispatching. The proposed approaches of conflict detection and resolution, including the rigid mathematical formulations, are imported to the agent model to assist the basic dispatching tasks and decision making.

In the applications of ERTMS/ETCS system, the inevitability of the influence on CDR functions that caused by different ERTMS application levels and the necessity of adapting the CDR to different ERTMS application levels are discussed. Some key 
factors that may have significant impact on the application of each ERTMS level are taken into account. Furthermore, a level adapter is proposed to add to the agent-based CDR support system for the ERTMS system, to allow the appropriate CDR approaches to be applied in a specific ERTMS/ETCS level or in the situations where the ERTMS/ETCS level transfers.

\subsection{Outline of the thesis}

This section gives an overview of the contents of each chapter. Figure 1-4 also shows an overall organization of the thesis and the relationships among chapters.

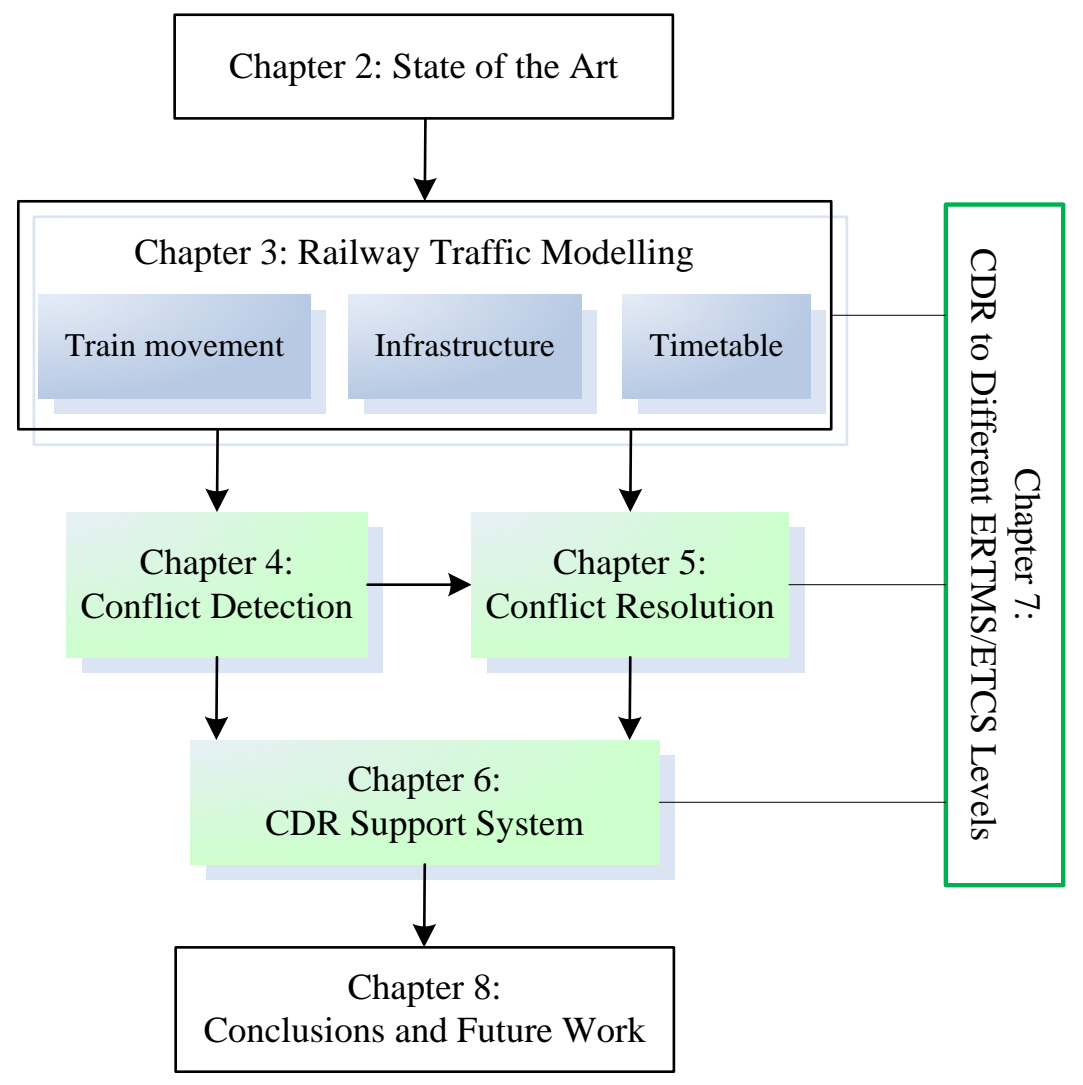

Figure 1-4 Structure of the thesis

Chapter 2 gives an overview of the related work of conflict detection and resolution in railway traffic management system. Existing systematic CDR support systems or equivalent tools are investigated.

Chapter 3 presents the railway traffic model by an integration of three partial models: train movement, infrastructure and timetable.

Chapter 4 and Chapter 5 focus on the algorithms on conflict detection and resolution.

Chapter 6 describes the design and realisation of an agent-based CDR support system for railway traffic management. 
Chapter 7 provides a comparison of CDR realisation in different ERTMS levels and analyses the corresponding effectiveness.

Chapter 8 concludes the main progress made in this thesis and briefly describes some research directions in the future work. 

Chapter 2

State of the art

An overview of conflict detection and resolution related work in railway traffic management is presented. 


\section{Chapter 2 State of the art}

In a wide sense, traffic conflict problems in railway involve infrastructure, signalling system, rolling stock, crew, passengers and all possibilities that may lead partial or the whole railway system into infeasible in a period of time if no effective measures are taken. Meanwhile, traffic conflicts may occur at any stage of the continuate railway operation process. The principal obstacles to study this kind of problem are the widespread domain and stochastic occurrences of the conflicts that increase the uncertainty of the problem in both space and time. A good amount of literature has rather mentioned or dedicated to solve some certain railway traffic conflicts than intensively collected and summarised all the contributions on railway traffic conflicts. Thus, the aim of this chapter is firstly to provide an overview of the researches on railway traffic conflicts and approaches on conflict detection and resolution in different stages of railway operation process.

In a real-time dispatching system, given the current infrastructure status, timetable, rolling stock information, the position and speed of each running train, to find potential conflicting train routes in a pre-established period of traffic prediction is called conflict detection (CD) procedure. Given the actual train delays and predicted conflicts, to propose how to solve them on the basis of the most suitable and robust dispatching options, e.g. train rescheduling, rerouting, removing some train runs, etc., is called conflict resolution (CR) procedure (Corman, 2010; D'Ariano, 2008). The main circular dispatching process includes monitoring and forecasting the operating situation in railway operation, identifying and solving disturbances in the traffic flow, and adjusting the train movement continuously (Cui, 2010; D'Ariano, 2008). A corresponding illustration of general conflict detection and resolution (CDR) support system in a realtime dispatching system is shown in Figure 2-1.

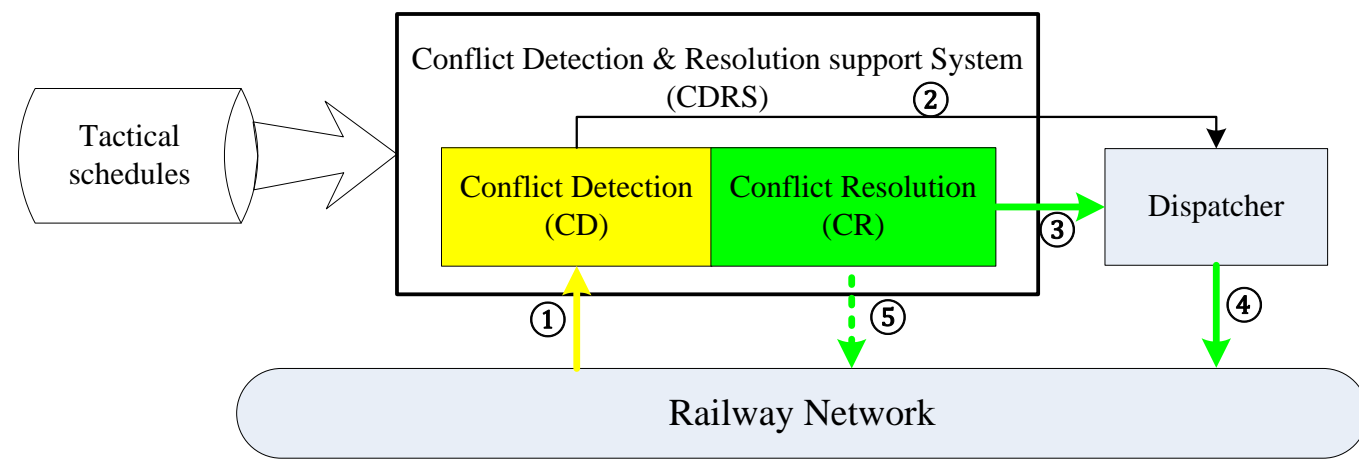

\footnotetext{
(1): monitoring and collecting real-time data from railway network

(2): alerting the dispatcher to potential conflicting situations

(3): information and resolution advice to the dispatcher

(4): adjustment commands of the decision made by the dispatcher

(5): possible automated adjustment made by conflict resolution procedure
}

Figure 2-1 Conflict detection and resolution in a dispatching system 
To design an automated CDR support system, Traffic Management System (TMS), Decision Support System (DSS), etc., are also surveyed in this chapter.

\subsection{Approaches of conflict detection}

What are the differences between conflicts and train delays? In railway traffic, the initial delay (primary delay) is caused by original failures and disturbances, while the consecutive delays (knock-on or secondary delays) are caused by the hinder from other trains or dispatching measures when solving potential train conflicts in the network (Corman et al., 2010; D'Ariano, 2008; D'Ariano et al., 2007a; D'Ariano et al., 2007b). These two types of delay absolutely constitute the total delay, which however is usually detected by the deviation of the train arrival time from the scheduled time at a relevant point in the network. The initial delay is a constant delay that is inevitable, while the consecutive delay is a variable delay that depends on the efficiency of conflict detection and resolution by traffic dispatchers or an automated traffic control system.

It is assumed that the basic timetable, an input of the dispatching system, is conflictfree, and other plans, e.g. rolling schedule, etc., are feasible in the initial condition, the detection procedure may principally involve three aspects: data collection, prediction and conflict identification.

\subsubsection{Data used in conflict detection}

Data in conflict detection contains two categories: restrictive information and facts that can be known in advance and the real-time data obtained in dynamic traffic flow.

Railway constraint preparation is indispensable in professional simulator development. It is aimed to be as analogic as possible. However, apart from in vivid simulation and some knowledge based approaches, it is rather difficult for the researchers to model all the details in mathematical approaches. There has been some excellent work in modelling railway traffic by formulations yet only for some particular aspects (e.g. scheduling and rescheduling) rather than the entire problem, typically such as (Carey and Crawford, 2007; Törnquist and Persson, 2007), representing the constraints in mixed-integer linear programming formulations for the purpose of optimizing delay situations, and (Lusby et al., 2013; Pacciarelli, 2002; Zwaneveld et al., 2001) applying graph theory (e.g. set packing, alternative graph, etc.) mainly to describe the network characteristics where nodes (vertices) and edges are combined to indicate route (in)compatibility. However, among them there are few models specially developed for conflict detection.

Data sources in conflict detection problem determine the calculation time requirement and the work pattern of applied approaches. Usually, historical records can be used to analyse the general rules and other complicate correlations in traffic that might be ignored in real-time traffic control. On the contrary, real-time collected data provides rich instant message but it allows a quite limited time for processing the data and 
making decisions. For example, the software tool TNV-Conflict presented in (Goverde et al., 2008) was able to identify all signalled route conflicts automatically using historical data, however, the on line implementation was not ensured for the short time slice of data collection and processing. Nevertheless, to a great extent the precision of real-time data collection depends on the detection devices, e.g. sensors of the safety, signalling system, etc., which are supposed reliable in this survey, thus the collected data, including infrastructure status, train positions, current speed profiles, etc. Instead, rapid and multi-dimensional data processing (data mining) methods are increasingly concerned.

\subsubsection{Conflict prediction}

Conflict prediction is always accompanied by uncertainty for its future projection feature as well as for the stochastic characteristic of traffic itself. A number of references proposing commendable approaches in delay propagation and train movement prediction may inspire the approaches in conflict detection. According to the instant starting the periodic conflict detection procedure, we categorise methods for conflict prediction into two groups (Case 1 and Case 2), with the only cut-off point of time detecting the initial delay.

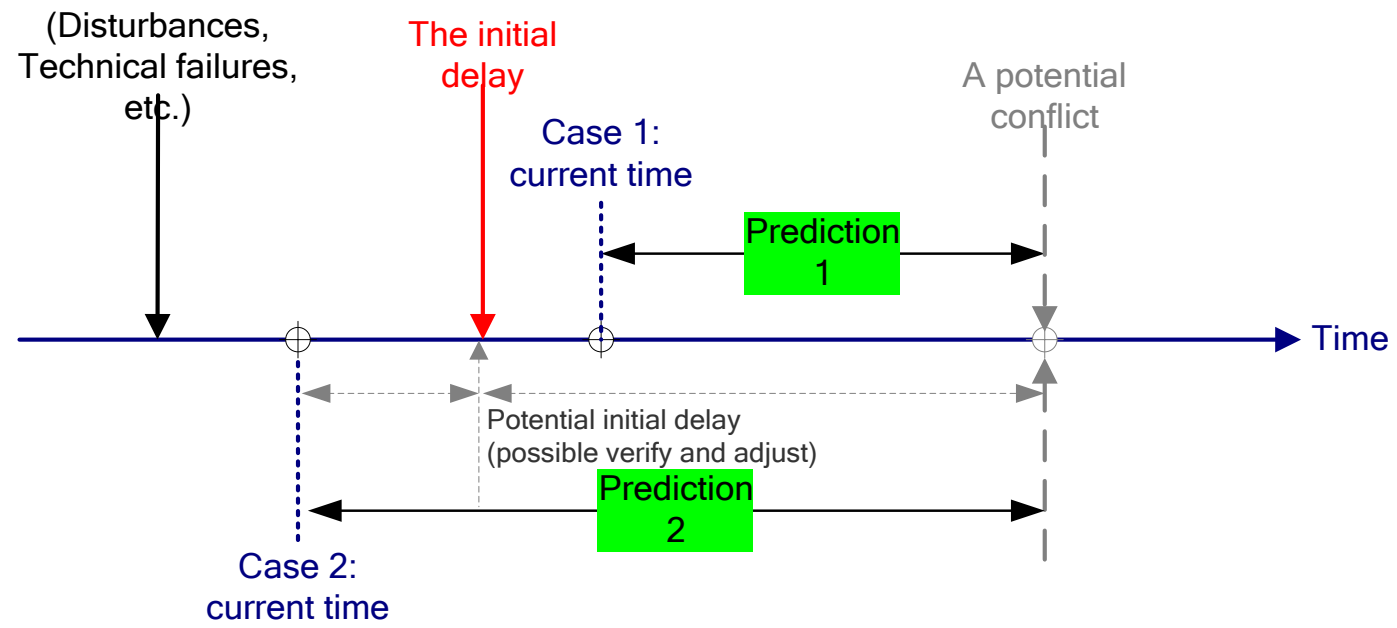

Figure 2-2 Prediction periods according to variant detecting (start) time

The first group represents the methods used to predict any potential conflicts after the initial delay have been detected (Case 1 in Figure 2-2). Predictions of delay propagation over a railway network fall into this category, such as work in (Goverde, 2007) where a delay propagation model in max-plus algebra theory was studied, delay propagation of initial delay scenarios over time and space could be computed by explicit recursive equations taking into account zero-order dynamics, later an updated model using a bucket-based delay propagation algorithm was proposed by the same author (Goverde, 2010) in order to reduce the memory usage and to avoid a huge amount of unnecessary computation, making the model suitable for online use in dispatching systems; in 
(Murali et al., 2010) a regression model was fitted on the collected data to develop the delay models for single and double-track networks; and analytical stochastic models of train delay propagation have been proposed to estimate the knock-on (secondary) delays of trains, with the constraints of signalling system and train protection operations rules taken into account (Huisman and Boucherie, 2001; Meester and Muns, 2007; Yuan and Hansen, 2007). The second group represents the prediction methods used before an initial delay occurs (Case 2 in Figure 2-2) or in an extra case, the initial delay is not detected (however, in fact that has occurred), i.e. without any data of primary delay or information of its cause. Nevertheless, abundant data of other static and dynamic parameters are required in this situation, e.g. the current infrastructure status, the position and speed of each train, limited speeds on current occasion, aimed timetable, train characteristics, etc. Train movement estimation and train blocking time prediction, where blocking time is composed of three time intervals: the time before the train passes the distant signal, the running time between the distant signal and the end of the block section and the release time (Hansen and Pachl, 2008), belong to this category. Besides, (Medeossi et al., 2011) presented a model of traffic conflicts in terms of probability to estimate delay generated by conflict. A main idea embedded in TNV-Prepare (one of Dutch train describer tools) is to use data from standard train describer records, enabling accurate calculation of train movements on track section occupancy level and estimate of arrival and departure times at platform tracks in complex railway stations (Goverde, 2005; Goverde and Hansen, 2000). Then the train speed profiles can be predicted, and the potential headway and the future location of trains can be estimated to predict whether there are potential conflicts forward (D'Ariano et al., 2007b).

The two groups of methods are not absolutely separated, so it is common that they are integrated in a hybrid model. Some methods may be applied in both cases in Figure 2-2, e.g. methods in case 2 can be applied in case 1 as well. The feature of the prediction models varies with particular emphases in different situations. The topologic structure of railway network is typically illustrated in the first group, while in the second group the real-time data of dynamic traffic behaviours are significantly to be exploited. However, it should be noted that most railway traffic prediction models have not been implemented in an online procedure of traffic conflict detection although they focus on dynamic characteristic and may have been studied in many cases based on historical train detection data in real railway systems.

\subsubsection{Conflict identification}

Conflict identification usually can be the direct result of traffic prediction, as well as a further description of the identified conflict, e.g. the type of the conflict, the position and possible causes, etc. When the prediction results are not definitely indicating the detailed information of conflicts, some mandatory and technological parameters in railway traffic can be studied. Thresholds of mandatory parameters are usually referred to the existing safety criterions and operational rules in railway, while that of technological parameters have to be concluded from concrete traffic operational 
implementation. For example, in COMBINE project, many experiments were carried out to analyse how technological parameters affect the performance of the traffic management system (TMS) under different values in dispatching the traffic. And it was arrived that the system could operate efficiently even when the absolute control loop delay was by 1 min (in ERTMS level 3), where the absolute control loop delay was the most importance technological parameter of TMS (Mazzarello and Ottaviani, 2007). So far, there is little literature declaring the absolute thresholds of parameters in train delay propagation models, due to the stochastic characteristic of the process and the variety of trains and corresponding control systems. Yet the distributions of the arrival and departure time (delay) of each train series at a station were estimated (Yuan and Hansen, 2007), based on a group of input distributions and deterministic parameters.

More commonly, conflicts are categorised into particular types and each type can be detected by verifying whether all relative constrains have been considered. In (Kim et al., 2011) line conflict and station conflict detection hierarchically categorised in detection, considering respective job of each layer. Considering high-speed railway in China, (Wen, 2010) classified the operational conflicts into 7 types, i.e. the station interval conflict, conflict of section occupation, utilization conflict of arrival and departure track, conflict between train operation and maintenance work, conflict between train operation and passengers' transfer time, connecting time conflict of electric multiple units (EMUs) and time conflict of cross-line trains entering high-speed lines, and they proposed a method by judging the derivation of train operation status circularly for each type of conflict. In the EU project COMBINE the types of conflicts that the designed system should be able to detect, isolate and attenuate were: deviations from plan, headway conflicts, passing conflicts, meeting conflicts, crossing conflicts, converging conflicts, locked tracks, fouling of gauge, order conflicts, route conflicts and dynamic schedule synchronization conflicts (Pentimelli and Marconi, 2001). In short, conflict detection could be considered as modelling the conflict problem, checking all the time the follow constraints: arrival and departure time constraints, minimum dwell time constraints, running time constraints, following and overtaking constraints, meet constraints, station/siding capacity constraints and possible constraints by traffic control systems.

To reduce the time consumption of conflict detection in a complex railway network, other methods of conflict detection used in optimising tactical timetabling or routing have also been introduced into the operational level, e.g. conflict graph (CG) model (Caimi et al., 2009; Zwaneveld et al., 1996), resource tree conflict graph (RTCG) model (Caimi et al., 2011; Caimi, 2009), etc.

\section{Identification by using the blocking time theory}

Blocking time theory (Hansen and Pachl, 2008) has been widely applied to the conflict identification. The main idea that the references on conflict detection and resolution stated is to identify the conflicts via "detecting the train number that steps 
into a block at a restricted signal (signal at the end of the block is at danger)" (Daamen et al., 2008; Hansen, 2009), or in a visible manner, by displaying the timetable in terms of blocking time plot and then a conflict in block can be observed from the blocking time plot (D'Ariano, 2008).

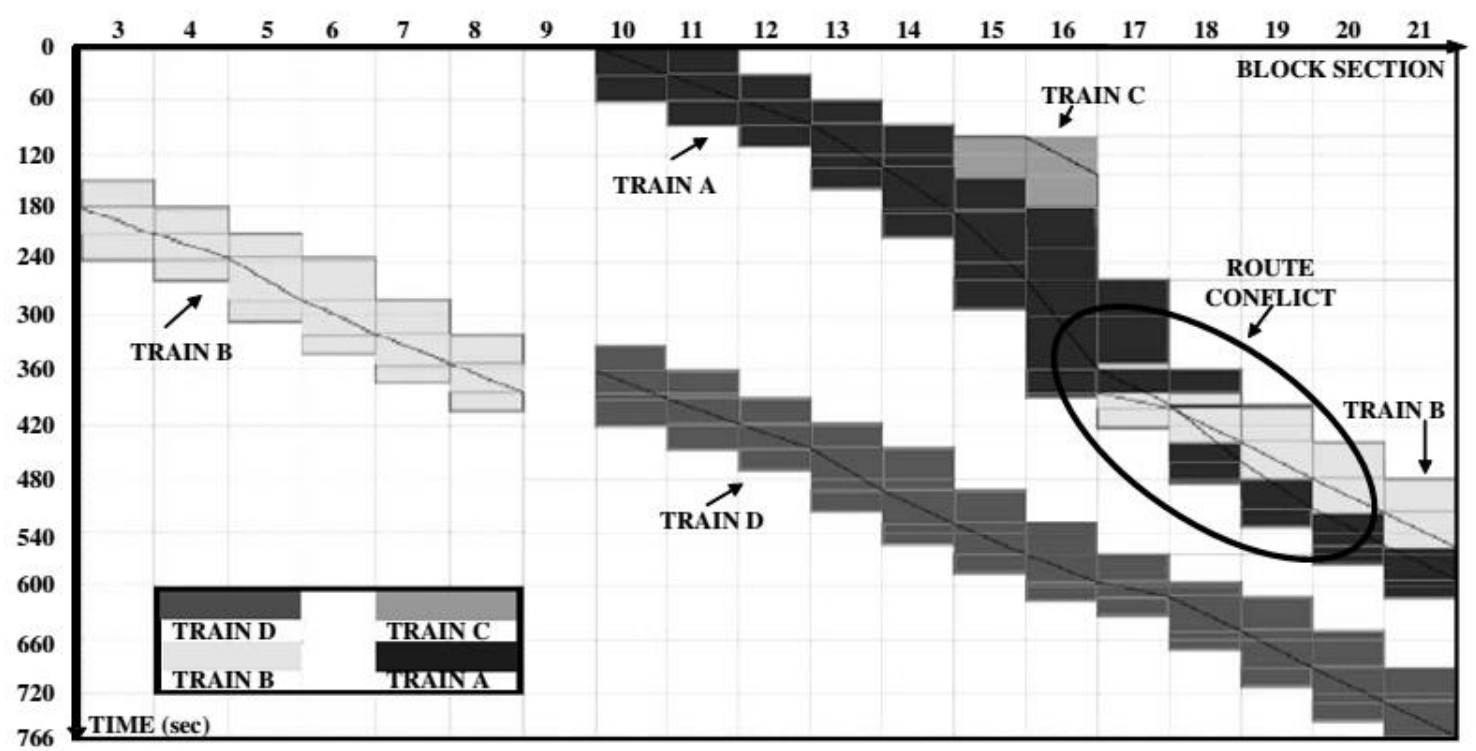

Figure 2-3 Route conflict detection between trains in time-space blocking time plot, figure from (D'Ariano, 2008)

The accuracy of the blocking time estimates depends on the available information. In station areas, full information of route blocks may be available, i.e., the passage time of the approach signal and the release time of switches, crossings, and route sections in yards and stations. On most open tracks however only partial information is available: neither track-clear detection nor signal states are logged and so they must be estimated from train number steps.

According to Daamen et al., 2008, the knock-on delays of individual trains, as well as the corresponding hindering train and the hindrance time due to this train can be automatically identified based on this theory.

\subsection{Approaches of conflict resolution}

Railways are typically operated according to a master timetable. This timetable represents a conflict-free coordination of train paths and includes slack times to manage train delays. So within the timetable design process, a timetable should be designed in such a way that it will be able to cope with small stochastic disturbances while the resolution of (unpredictable) large disruptions is left to real-time traffic management (D'Ariano, 2008). Properly, it is the issue of off-line timetable generation and optimisation. Some experience and principles are obtained in real operation of railway companies, such as the concept of improving the traffic management, especially in congested areas e.g. large stations and junctions, by planning less in the off-line 
timetabling phase and postponing the resolution of possible conflicts among trains to the real-time traffic dispatching (Schaafsma and Bartholomeus, 2007). Based on this consideration, D'Ariano et al. evaluated the benefits of flexible timetable in which less details are fixed in the plan and more control decisions are left to the dispatcher (D'Ariano et al., 2008b). A similar idea was stated in (Caimi, 2009), where the author extended a scheduling model called Periodic Event Scheduling Problem (PESP) into the Flexible Periodic Event Scheduling Problem (FPESP) and Flexbox model, allowing for time slots for each event instead of fixed times, then this flexible timetable can be as an input used in microscopic (operational) level. More overviews of timetable generation and train routing were presented in (Cordeau et al., 1998; Lusby et al., 2011a; Törnquist, 2005). Approaches applied in determining the initial timetable (or in other scheduling problems) are helpful in later rescheduling. However, approaches in tactical scheduling and operational rescheduling are not equivalent. One important reason may be the strict limit of time allowing man or control system to solve the problem when it appears suddenly. That is, if scheduling approaches are introduced into conflict resolution it is highly required that they should be able to respond quickly and exactly. Therefore, those off-line scheduling algorithms should not be ignored, but ways have to find to apply them into real-time procedures.

Considering the approaches on rescheduling and dispatching optimisation or conflict resolution, double trends have been obviously focused on in recent years. One is more detailed models of railway system and practical requirements, and the other is more efficient algorithms to achieve effective and reliable solutions (Corman et al., 2010).

\subsubsection{Problem modelling}

To describe the railway system topologies and control strategies in rescheduling or rerouting problems, mixed integer linear programming (MILP), alternative graph, etc., have been quite widely used in research.

\section{Mixed integer linear programming (MILP)}

Adenso-Díaz et al. (1999) as pioneers adopted a MILP model to maximise the number of passengers transported when considering the problem of real-time timetable perturbations management in a regional dispatching area. As a result, a conflict resolution system using the MILP model and backtracking heuristic techniques was implemented to support dispatchers at a traffic control centre of the Spanish national railway company, Renfe.

Dessouky et al. (2006) developed a detailed MILP formulation for determining the optimal dispatching times for the trains traveling in a complex rail network. According to their work, a railway network that consists of main tracks, sidings, junctions, and platforms, can be generally formulated as a graph, which contains a node set and an arc set. Each node contains a set of segment resources and a set of junction resources and each arc, contains no segment or junction resources. They let the length of node be a 
positive value, and let the length of the arc be zero. For each train its length and path from the origin node to the destination node are describable. Constraints such as the earliest time that a train's head can arrive at a node, the latest time its tail has to leave node, minimal travel time, the minimal safety headway, etc., were used to assure that the behaviours of a train as logical as possible. Zero-one decision variables were defined to represent the sequence of two trains passing the same nodes. The minimised total arrival time to all destination nodes was sought in this MILP formulation.

To investigate more aspects of the rescheduling problem, (Carey and Crawford, 2007; Törnquist and Persson, 2005; Törnquist and Persson, 2007) presented another MILP formulation to represent a more complex railway network which can be mixed composed of a large number of tracks (n-tracked network), unidirectional as well as bidirectional, and with other individual properties. Besides the train set and segment set, a set of events were also defined to relate the train and resource, i.e. an event is a resource request by a certain train for a specific segment. According to the original timetable, event sequence and the constraints of each event were expressed in this MILP model. In addition to the technical restrictions of trains, segments and preferences of operators, Törnquist et al. also considered the interdependences between events, e.g. two trains scheduled to meet and exchange passengers. In train delayed situations, to get a conflict-free schedule, two objectives were considered in the formulation of Törnquist and Persson, 2007. The basic one is to minimise the sum of the final delays when trains arrive at their final destination, i.e. the total final delay. The other is to minimise the total final cost associated with delays where a penalty mechanism was introduced to calculate the additional cost when the delay exceeds a certain degree. Based on the MILP formulation proposed, they dedicated to develop a good solution to the problem for large and real-world scenarios.

\section{Alternative graph formulation}

Alternative graph formulation is another effective method to describe the scheduling and rescheduling problem with complex railway network. An alternative graph formulation was firstly proposed to address the scheduling problem for a set of jobs in a job-shop environment with blocking and/or no-wait constraint, and was found to be a powerful discrete optimisation tool, especially in dealing with scheduling problems (Mascis and Pacciarelli, 2000, 2002). They found that the combinatorial structure of the train scheduling problem was similar to that of blocking job shop scheduling problem, with a block section (track segment) corresponding to a blocking machine and a train corresponding to a job. We can make a comparison of these two kinds of scheduling problems by Table 2-1. It shows that the approaches to model and solve the blocking job shop scheduling can also be applied in railway train scheduling problem. Thus they innovatively implemented the alternative graph formulation to optimise the railway traffic scheduling and extended it to the conflict resolution in dispatching (Mascis et al., 2002; Mascis et al., 2001). 
As defined (Corman, 2010; Corman et al., 2010; D'Ariano et al., 2007a; Mascis and Pacciarelli, 2002; Mascis et al., 2002), an alternative graph is a triple $G=(N, F, A)$, where $N$ is a set of nodes, $F$ is a set of fixed directed arcs, and $A$ is a set of pairs of alternative directed arcs (Mascis et al., 2002). A node of an alternative graph is the passing of a train through a particular block section (an operation). A fixed arc is a fixed precedence relation (given two consecutive nodes $j$ and $i$ in the sequence, $j$ can only start $f_{i j}$ time units later after the completion of $\left.i\right)$, denoted as $\operatorname{arc}(i, j) \in F$, with a weight $f_{i j}$. An alternative arc $(i, j)$ or $(h, k)$ represents an alternative precedence relation (given two consecutive node pairs $(i, j)$ and $(h, k)$ with $j$ and $\mathrm{k}$ are conflicting that can be considered to be disjunctive), denoted as arc $(i, j) \in((i, j),(h, k)) \in A$ and $(h, k) \in((i, j),(h, k)) \in A$, respectively with the weight $f_{i j}$ and $f_{h k}$. A schedule is feasible when all fixed precedence relations and exactly one for each pair of the alternative precedence relations are satisfied.

Table 2-1 A comparison of problem description in job shop and in railway traffic scheduling

\begin{tabular}{|c|c|c|}
\hline No. & In job shop problem & In traffic scheduling and CR problem \\
\hline 1 & A job & A train \\
\hline 2 & A machine & A track segment (block section) \\
\hline 3 & $\begin{array}{l}\text { An operation: the processing of a job } \\
\text { on a machine }\end{array}$ & $\begin{array}{l}\text { An operation: the passing of a train through a } \\
\text { particular track segment }\end{array}$ \\
\hline 4 & $\begin{array}{l}\text { A job: } \\
\text { (1) must be processed on a set of } \\
\text { machines } \\
\text { (2) the sequence of machines for } \\
\text { each job is prescribed }\end{array}$ & $\begin{array}{l}\text { A train: } \\
\text { (1) must pass through a given set of track } \\
\text { segments } \\
\text { (2) the sequence of track segments to be } \\
\text { traversed for each train has offline } \\
\text { developed in timetable (path for each train) }\end{array}$ \\
\hline 5 & $\begin{array}{l}\text { A set of } n \text { operations have to be } \\
\text { performed on } m \text { machines. }\end{array}$ & $\begin{array}{l}\text { A sequence of } n \text { operations to be executed by } \\
\text { the train, passing } m \text { block sections, in order to } \\
\text { reach its destination (route of a train). }\end{array}$ \\
\hline 6 & $\begin{array}{l}\text { Each operation requires a specified } \\
\text { amount of processing time on a } \\
\text { specified machine. }\end{array}$ & $\begin{array}{l}\text { Each operation requires a given running time } \\
\text { to pass a block section. }\end{array}$ \\
\hline 7 & $\begin{array}{l}\text { Each machine can process only one } \\
\text { operation at a time. }\end{array}$ & $\begin{array}{l}\text { Each block section can host at most one train } \\
\text { at a time. }\end{array}$ \\
\hline 8 & $\begin{array}{l}\text { An operation cannot be interrupted } \\
\text { from its starting time to its completion } \\
\text { time. }\end{array}$ & $\begin{array}{l}\text { A train cannot disappear suddenly from a } \\
\text { track segment from its starting to its } \\
\text { completing passing on the segment. }\end{array}$ \\
\hline 9 & $\begin{array}{l}\text { Time lag: a given time lag between } \\
\text { consecutive operations can be } \\
\text { positive, null or negative. }\end{array}$ & $\begin{array}{l}\text { Setup time: an additional safety margin, } \\
\text { between the exit of a train from a block } \\
\text { section and the entrance of the subsequent } \\
\text { train in the same block section. }\end{array}$ \\
\hline 10 & $\begin{array}{l}\text { A precedence relation: a constraint } \\
\text { between the starting times of the } \\
\text { sequential operations. }\end{array}$ & $\begin{array}{l}\text { A constraint for operations transferred from } \\
\text { the requirement of (minimum) separation } \\
\text { among trains. }\end{array}$ \\
\hline 11 & $\begin{array}{l}\text { A schedule: an assignment of starting } \\
\text { times of operations. }\end{array}$ & $\begin{array}{l}\text { A schedule: a timetable with all the arrival and } \\
\text { departure times of each train at each stations }\end{array}$ \\
\hline
\end{tabular}


In the graph, the weight can be considered as the length of a fixed arc or an alternative arc. A graph selection $S$ is a set of alternative $\operatorname{arcs}$ chosen from $A$, by choosing at most one arc from each pair, denoted $G(S)=(N, F \cup S)$. The selection $S$ is complete if exactly one arc from each pair is chosen, while it is consistent if the graph $G(S)$ has no positive length cycles. Mascis et al arrived at the conclusion that each schedule could be associated with a complete consistent selection on the corresponding alternative graph.

The alternative graph allows formulation to set various objectives in different problems. For example, D'Ariano et al. (2007a) proved that the longest path $l^{S}(0, n)$ could represent the maximum secondary delay of the associated schedule where the value of a longest path from $i$ to $j$ in $G(S)$ was denoted as $\mathrm{l}^{\mathrm{S}}(i, j)$; and the minimization of secondary (consecutive) delays for all trains could be one of the most common objective functions when an initial delay occurs in the network (D'Ariano, 2008). With a similar structure, off-line conflicts can be detected by means of a topological visit of the alternative graph, and real-time conflicts within a given time window can also be solved by adding the alternative pairs to the graph representing the conflicts and searching a feasible arcs. Moreover, the formulation still can easily include other constraints relevant to the railway practice without making it more complex. A following constraint between two trains at a station, where one train must wait for the arrival of another before departing, can be realised by simply adding one fixed arc with suitable weight from the node associated to the arrival of the former train to the node associated to the departure of the latter one. A meeting constraint specified a minimum dwell time for two given trains to exchange passengers or goods can be modelled by adding two fixed arcs with suitable weights. One arc is from the node associated with the arrival of the former train to the node associated with the departure of the latter train and another arc is from the node associated with the arrival of the latter train to the node associated to the departure of the former train.

Other models have also been proposed, such as set packing models (Lusby et al., 2011b; Zwaneveld et al., 1996; Zwaneveld et al., 2001), discrete-event models (Dorfman and Medanic, 2004), etc. According to the eventual characteristic of conflicts, discrete event simulation methods with the discrete state changing on each time events are extended, e.g. Petri net, Markov chain and queuing theory, etc.

\subsubsection{Algorithms on conflict resolution}

Algorithms may differ from the types of conflict (and even the definitions of a conflict).

\section{Strategies and heuristics}

Integrated with the models formulated above, further strategies and heuristics are flexibly applied to solve the problems and improve the performance of each single model. 
In Törnquist and Persson (2007), strategies such as Allow Swaps of Tracks but Maintain Order, Allow Swaps of Tracks and Implicit Change of Order, Allow a Certain Number of Order Swaps for Specific Segments and Allow All Changes etc., were applied to calculate the complete MILP formulation, with considering the characteristic of railway rescheduling problem that the main part of the sequence of trains on the segments specified by the initial timetable remains the same, only a few modifications may be necessary to achieve a significant improvement. The strategies were formulated by additional constraints. Before that, another two heuristics, Tabu Search (TS) and Simulated Annealing (SA), had been used in the same MILP model (Törnquist and Persson, 2005). The experimental results showed that the heuristic approaches used for rescheduling in a geographically large and fine-grained railway network with highly interacting traffic had a good computational capability.

Based on the longest path in the alternative graph model, heuristics were also further applied in the problem, e.g. Avoid Maximum Current Cmax, Select Most Critical Pair, Select Most Balanced Pair, etc., were proposed in (Mascis and Pacciarelli, 2002). With the alternative graph formulation, branch and bound algorithm for scheduling and rescheduling (D’Ariano et al., 2007a; Mascis and Pacciarelli, 2002) and local searching algorithm for routing and rerouting (D'Ariano et al., 2008a) were developed. Furthermore, dynamically train speed adjustment approaches in traffic control loop (D'Ariano and Albrecht, 2006; D'Ariano et al., 2007b) and an additional green wave strategy (Corman et al., 2009) were applied in real-time optimisation. Interestingly, the very searching algorithm tabu search (TS) has also been applied in alternative graph model to resolve the conflicts (Corman et al., 2010; D'Ariano, 2008; Pacciarelli and Pranzo, 2001). Meanwhile almost all the experiment results, including that in (Törnquist and Persson, 2005), showed that TS could significantly reduce train delays, generally performing better than or equal to other local searching heuristics.

We can also find that Branch and bound (B\&B) has been adopted in both MILP and alternative graph models, to solve both scheduling optimisation and rerouting problems. Some earliest work to solve the problem of scheduling trains with B\&B algorithms was carried out in (Carlier and Pinson, 1989, 1994; Szpigel, 1973). The algorithm was addressed for rescheduling trains in conflict problems by (Higgins et al., 1996). Since it has the advantage of searching the entire space of candidate solutions by using previous estimates on the quantity being optimised to bound out large parts of the search space, as a result, reducing the computation time efficiently, $\mathrm{B} \& \mathrm{~B}$ algorithms have been developed based on various models (D'Ariano et al., 2007a; Dessouky et al., 2006; Mascis and Pacciarelli, 2002; Törnquist and Persson, 2005; Törnquist and Persson, 2007). Looking into the reasons, firstly, B\&B has its clear steps to work on graph and tree search problems which can be desirably used to express the topologies of railway network; secondly, B\&B can be a base of search strategies obtained from practical dispatching rules in railway. To be the key factors, upper and lower bounds are always discussed in developing good or fast enough B\&B algorithms. 


\section{Meta-heuristics and intelligent algorithms}

In addition, meta-heuristic and intelligent algorithms are introduced to railway conflict resolution, while adopted approaches are further researched to increase the solution quality and reduce the computation resource.

A procedure based on the application of genetic algorithm (GA) technique was introduced to solve the railway junction conflict resolution problem in (Ho and Yeung, 2000; Yeung and Ho, 2000). In junction conflict problem, each possible route sequence was modelled as a chromosome, and a proper set of chromosomes taken from the possible pool formed the population of the first generation. Then two basic steps to evolve through the generations, selection and replacement were: deciding the chromosome in the population deemed to be fit to produce off-springs and allowing the chromosome with the worst fitness to vanish in order to make room for the better offsprings to compete. As GA still could not guarantee a global solution, stricter neighbourhood definitions were proposed to optimise local search procedures to ensure few worse chromosomes in a new generation than those in the previous generations. There was always a trade-off between the satisfaction of solution and the computation time, especially related with the string length, population size and termination condition.

Knowledge-based systems are also used to deal with the problems in conflict situations. Cheng (1996) applied a method of using a knowledge-based system combined with the critical path method for getting a nearly optimum reschedule under time and resource constraints, with three dispatching strategies considered in resolving conflicts: the prior arrival, local optimal strategy and critical path methods (CPM). Chiang et al. (1998) used a knowledge-based system to develop an automatic scheduling machine. By using the conflict-resolution knowledge extracted from the human experts, the system selects solutions for all conflicts in order to generate a conflict-free schedule. Expert systems have been popularly developed when the natural language "IF-THEN" spreads. (Araya and Fukumori, 1984) may be one of the earliest publications applying an expert system method to the decision support system design in railway traffic system, where a rule-based decision support system named ESTRAC-II was introduced for train traffic control in disturbed situations. This system could automatically generate a rescheduled plan after traffic disturbances, which applies many kinds of schedule adjustments, as human experts work manually. Another traffic control system based on conflict dispatching system was illustrated in (Schaefer and Pferdmenges, 1994), where an expert system provided the intelligent support for automatic conflict detection and resolution.

\section{Hybrid approaches}

Comparing the algorithms and models proposed, we may conclude that usually only one algorithm is not able to satisfy all objectives. Thus, hybrid algorithms and models have been proposed to integrate the merits of each single algorithm. A greedy rulebased, distributed intelligent search method was reported after a comparative analysis of 
several algorithm in project MARCO (Mazzarello and Copello, 2000), in which the search for a feasible solution of a given traffic problem was accomplished by the integration between local solving strategies and global optimisation criteria. Based on a similar discussing of the existing methods: first-come-first-served (FCFS), genetic algorithm, simulated annealing, tabu search and ant colony for a standard junction conflict problem, Fan et al. (2011) addressed a hybrid algorithm combining decision tree-based ant colony with a simple local search for optimal junction traffic control. They reached a result that the hybrid algorithm could get closer to the optimal solution with a favourable computation time compared to other methods. Another example was the combination of fuzzy Petri Net approach and expert system in (Cheng and Yang, 2009; Fay, 2000), where the former was adopted to formulate the dispatcher's expertise knowledge rules.

Since train traffic control includes large-scale dynamic and combinatorial problems that is hard to be analysed by conventional approaches, system approaches are usually applied as well, i.e. when a problem is large-scale and complex, a system approach views it as a part of an overall system rather than an isolated entity. In recent years, flexible simulation and distributed intelligent decision support systems have been considered useful in solving these problems (Lu et al., 2004; Murali et al., 2010).

\subsection{Systematic CDR support Tools}

Some tools were not originally designed for conflict resolution, but they played a great reference role in CDR support tool development, e.g. DONS (Designer Of Network Schedules) is the Dutch timetable design system which generates a timetable on a macroscopic level and checks the feasibility of timetable on a microscopic level (Hooghiemstra et al., 1999). And Caimi (2009) extended and improved the models and algorithms used in DONS, with the ultimate goal of ensuring that the microscopic schedule is conflict-free on the blocking times level for all parts of the railway network.

\subsubsection{CDR tools in MARCO}

The exact research programme on conflict detection and resolution MARCO (Multilevel Advanced Railways Conflict resolution and Operation) was one of the projects of the Rail TAP-Transport Area during 1996 to 1999 (D'Addio et al., 1998; Pentimelli and Marconi, 2001). The MARCO project was pioneering to develop tools, algorithms and technology for Conflict Detection and Resolution under unpredictable disturbances to existing train schedules, in a wide range of real time applications within railway and metro networks (including complex junctions, stations, main and local lines). Its main results concluded a set of tools for Conflict Detection and Resolution and specific Man-Machine Interfaces (MMI).

Significantly, in this project a number of algorithms (branch and bound, branch and cut, random search, GENET, intelligent search, distributed intelligent search, simulated annealing, genetic algorithms) had been tested on realistic test cases. As a result, it 
turned out that for junction demonstrators used, two different variants of greedy rulebased methods (intelligent search and distributed intelligent search) were proved to be the best, with a number of indicators (resolution and computing performances, conflict management capability, etc.) taking into account. It reported that the application of advanced tools for conflict detection and resolution would improve the use of congested railway networks, allowing the same network to have a higher throughput, offering better customer services by better prediction of expected train delays, reducing unexpected conflict situations and allowing faster and more efficient recovery of perturbed timetables, etc. The same tools could be used to perform simulations and forecasts, to analyse the impact of predictable types of disturbances on train schedules and to assess corrective measures.

\subsubsection{TMS in COMBINEs}

Based on the various results of MARCO, a followed Traffic Management System (TMS) was developed under the subsequent EU projects, named COMBINE (Giannettoni and Savio, 2002; Giuliari et al., 2000) and COMBINE 2 (Giannettoni and Savio, 2004).

The first COMBINE project focused on real-time optimisation of rail traffic in areas equipped with moving block safety system compliant with ERTMS/ETCS Level 3. The TMS Demonstrator was developed based on a multi-layer and modular system architecture, in order to make it potentially suitable for any ERTMS/ETCS compliant system and for railway networks of any size. Hierarchically, the highest level Conflict Resolution System level 2 (CRS2) controls the whole area helping a Dispatcher to monitor the system and make decisions; the medial level Conflict Resolution System level 1 (CRS1) detects and solves conflicts locally; and the foundational level of Speed Regulator (SR) controls the train movements in a moving block environment, by means of either advisory speeds or targets. Through the researches in the project and the development of TMS, the knowledge on railway operation in the ERTMS/ETCS level 3 environment was increased, and a list of the functional and performance requirements on a railway traffic conflict detection and resolution system were generated (Mascis et al., 2001). These requirements were based on an identification of the different types of users of the system as well as a functional analysis of their needs and of system constraints.

Consequently, COMBINE 2 project expanded the TMS architecture and capabilities in order to optimise the rail traffic in large railway networks equipped with mixed signalling systems. To fit any size of the railway network, the complete TMS architecture was designed to compose of a number of local TMSs. Each area is controlled by a local TMS, and several local TMSs are coordinated at a higher hierarchical level. New algorithms were devised for information exchange and collaboration in taking decisions between adjacent TMSs when managing interaction among TMSs controlling adjacent railway areas. A further detailed description of each 
TMS was stated in (Mazzarello and Ottaviani, 2007), where two core modules of the TMS, Conflict Detection and Resolution (CDR) of responsibility for automatic realtime train scheduling and routing and Speed Profile Generator (SPG) of responsibility for plan execution, were concretely introduced. Besides, as reported, the cooperation between CDR and SPG was implemented in the real pilot "The Green Wave", carried out on the Dutch railway routes Roosendaal-Dordrecht and Breda-Dordrecht.

\subsubsection{ROMA dispatching support system}

ROMA (Railway traffic Optimisation by Means of Alternative graphs) is an innovative laboratory tool for railway traffic management, developed by the "Aut.Or.I.", Research Group of Roma Tre University. D'Ariano et al. (2007b) indicated that this dispatching support system could be considered as part of a Decision Support System (DSS) for aiding dispatchers to control the train traffic. Two doctoral dissertations (D'Ariano, 2008) and (Corman, 2010) have been successively dedicated to the work of ROMA, as well as detailed presentation of this support system.

As named, ROMA is a tool developed on alternative graph modelling methods. To address complex multi-objective problems, algorithms such as B\&B, TS and greedy heuristics have also been applied in ROMA. It can deal with multi-level sub-problems in real-time dispatching. In the case of a timetable that is not conflict-free, the computation of a flexible conflict-free timetable is available; when train operations are perturbed, a new timetable of feasible arrival and departure times can be recomputed; the real-time traffic optimisation procedure can further take actions, e.g. train reordering and local rerouting, to solve the conflict detection and resolution problem; simultaneously, a train speed coordination procedure takes into account the train dynamics to satisfy minimum distance headways. To conclude, ROMA can recover from disturbances automatically, reduce the propagation of consecutive delays and improve the dynamic use of railway infrastructure.

Computational experiments on ROMA have been carried out on some dispatching areas of the Dutch railway network, e.g., the Schiphol railway bottleneck and the dispatching area between Utrecht and Den Bosch, etc.

\subsubsection{TNV tools}

Another set of tools are TNV series including TNV-Prepare, TNV-Conflict and TNVStatistics, etc., where TNV stands for the Dutch abbreviation of 'Train Number Following'. In the Netherlands, the train describer is implemented as the so-called TNV-system, with corresponding log files named TNV-logfiles.

TNV-Prepare derives detailed information of event times associated to train services from TNV-logfiles and generates the detailed data in the form of TNV-tables which are suitable for further data analysis and visible export (Goverde, 2005; Goverde and Hansen, 2000). TNV-Conflict automatically realises the data mining by using signalling 
logic and blocking time theories and detects route conflicts including the detailed information of critical track sections, involved train numbers, and the amount of separately initial and consecutive delay at a precision of less than 5 seconds (Goverde et al., 2008). Subsequently, Goverde and Meng (2011) presented TNV-Statistics, an addon tool to TNV-Conflict. It reuses the data generated by TNV-Conflict to obtain advanced information in secondary delays, route conflicts and associated operations. Besides, it still supports to relieve the analyst from routine data processing tasks, which are time-consuming and error-prone. A simplified relationship illustration of TNV tools is shown in Figure 2-4.

Since 2009, TNV has been gradually replaced by the new system TROTS (Train Observation and Tracking System). Accordingly, TNV tools have also been redeveloped based on TROTS log files. Real data from Dutch train describers has been used for analysis and test in the development of these tools. So far all TNV tools are in off-line versions, while an on-line version has been presented to be in consideration, which is intended to be used as a decision support system by dispatchers (Daamen et al., 2009).

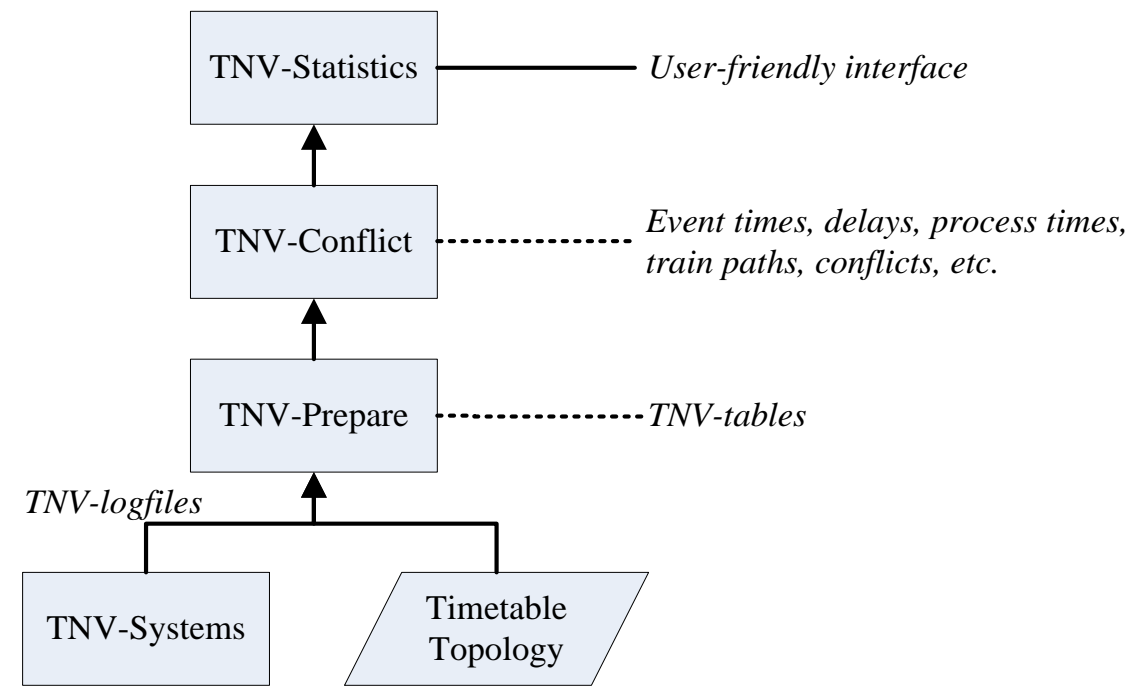

Figure 2-4 The relationship of TNV tools

\subsection{Discussions}

We can observe that no matter the theoretical research on conflict detection and resolution or the development of CDR tools, all the approaches adopted are evolving in the way closely connected with the technologies of search, artificial intelligence and simulation. Moreover, some trends are gradually emerging with the increasingly considering of the complete CDR problem.

\subsubsection{Research trends}

Take the research and development in several research groups as an example. As reviewed in section 2.2, the alternative graph formulation and a good many of 
algorithms based on it have been developed to solve scheduling and CDR problems. If we review their partial academic publications in chronological order, we can find the work around these problems have been taken out by the research group in Roma Technology University and Delft Technology University, besides the work during the last decade can at least be divided into three stages:

(1) Mascis and Pacciarelli, et al. firstly introduced the model of alternative graph from job shop scheduling to railway scheduling problems. They proposed some influential algorithms in railway traffic problems, started to expound and prove a set of heuristics according to railway dispatching rules. As participants in the successive projects COMBINE and COMBINE2, they took part in the design and development of the incipient TMS and DSS (see subsection 2.3.2), which was significant experience and reference to present and develop the follow-up support system.

(2) D'Ariano et al. made out a more detailed analysis about the railway traffic management and dispatching system, acceded and developed the alternative graph model and algorithms based on the work done in the former stage, with more concrete arguments, examples and experimental results. Targeted to develop a decision support system for real-time traffic management, multi-layer sub-problems including flexible scheduling, local routing and train speed profile adjustment, etc. were closely investigated. They fundamentally constructed the functional structure of ROMA (see subsection 2.3.3).

(3) As the inheritance and also integration of all advanced models and methods developed by the group, Corman et al. emphasised on CDR and dispatching problems in network and complicated station/junction areas, thus issues e.g. centralized and distributed dispatching methods, multi-class and inter-area rescheduling, bi-objective CDR, were particularly discussed. Meanwhile ROMA was extended and updated via integrating new algorithms and methods (Samà et al., 2016b).

It indicates that besides of well-fit models and advanced algorithms, dynamic characteristic of CDR problems, network resource distribution and cooperation management, multi-objective optimisation in CDR procedures and systematic simulation, etc. all have been the recent focuses or further research directions. In addition, effective evaluation of the resolutions and decisions in CDR problems can also be an issue of interest and challenge.

\subsubsection{Methodology in research}

As stated in 1.3, building an automated CDR module to support the railway traffic control and management system is one definite objective of this research project. To realise its fundamental functions as well as to get its innovative advantages, an applicative model has firstly to be established, which is the base and clue of all algorithms studied and developed in the later research. Then applied algorithms and 
methods have to be integrated and assessed before the module is developed. In order to achieve a certain level of automation, system design and testing of CDR module also need to be taken out, including its communications in the whole railway system. Figure 2-5 shows a schematic of introducing system approaches in our work on CDR research and development.

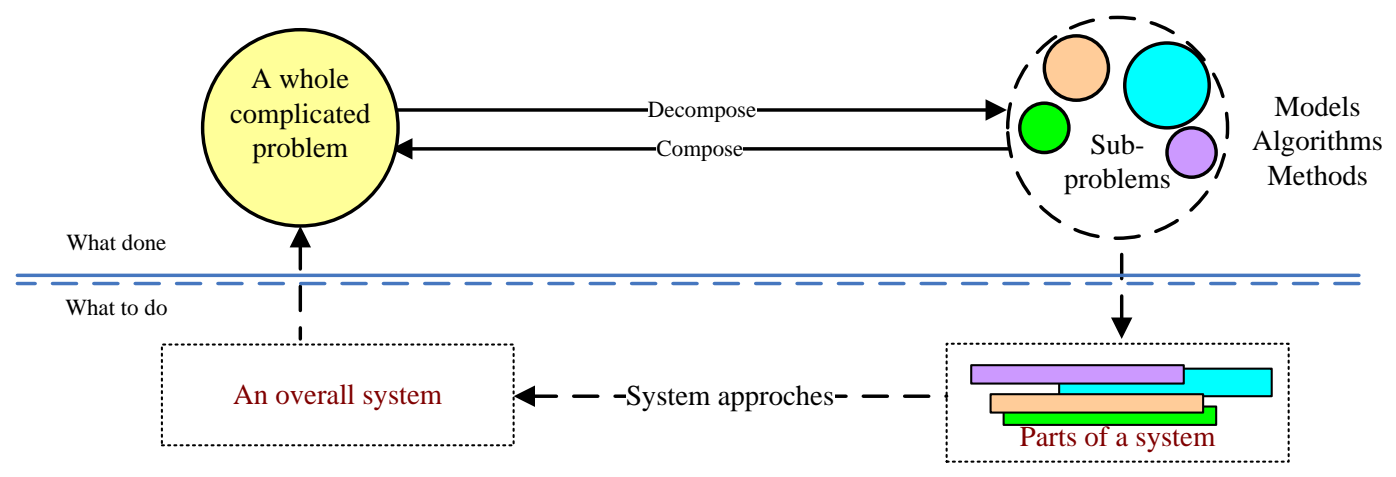

Figure 2-5 Previous work reviewed and future work schemed

Partial models such as data-based train movement model, infrastructure state transition model and timetable (rescheduled timetable) representation, are firstly established. Based on the models, conflict detection approaches and conflict resolution approaches are studied, respectively. An agent-based CDR support system integrates the data, knowledge and methods of CD and CR procedures. Finally, the realisation of CDR functions in different ERTMS application levels is further discussed and evaluated. 

Chapter 3

\section{Railway traffic modelling}

Three data-based models corresponding to train, infrastructure and timetable are introduced to represent the railway traffic. 


\section{Chapter 3 Railway traffic modelling}

Main models of railway traffic related subsystems are presented in this chapter. These data-based models mainly compose the environment of the conflict detection and resolution problem, not exactly being components of the conflict detection and resolution support system.

Figure 1-2 shows the relations that the traffic management system (or ETML in ERTMS system) has with the train control system ERTMS/ETCS and the underlying system. Correspondingly, the representative models of train and infrastructure, especially their dynamic characteristics, are concerned in this chapter, as well the representation of timetable that plays a foundational role in organizing the trains and underlying infrastructure.

\subsection{Terminologies}

In addition to some concept definition in previous chapters, e.g. initial delay, secondary delays, total delay, etc., some related terminologies and symbols are defined in this section. Meanwhile, some literatures are partially referenced (Corman et al., 2010; Min et al., 2011; Törnquist and Persson, 2007).

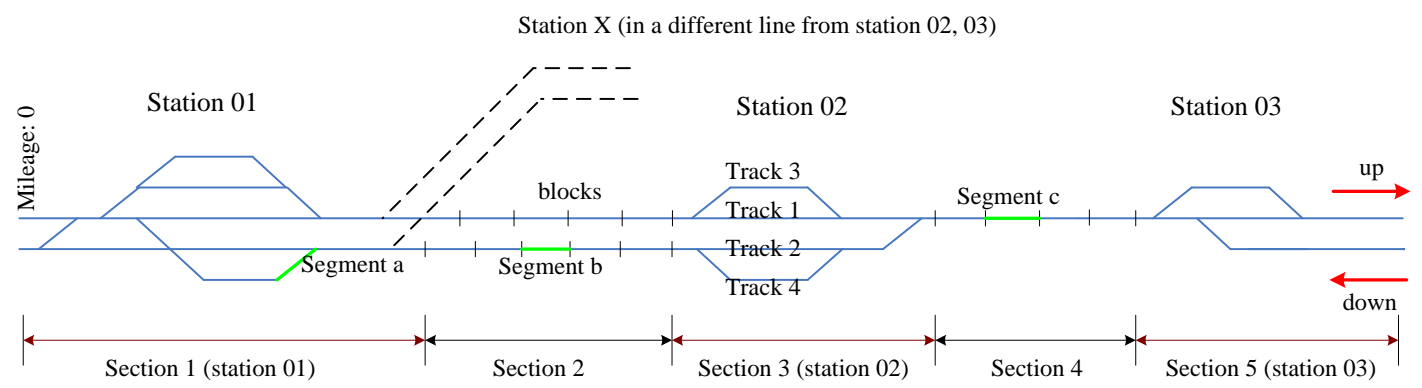

Figure 3-1 An illustration of a railway line (from station 01 to station 03) and network

Line: a line is generally named to describe a continuous section of railway infrastructure that connects two or more destinations. Two or more intersecting railway lines compose a railway network.

Track: the track is the general structure for train to move that composes the railway line or railway network. In order to indicate lateral relations inside the infrastructure topology, tracks could be named in indexed numbers and prescribed by endpoints.

In station areas, there are commonly two or more than two tracks for trains to stop and realise other operations. Besides, sections or segments between stations are usually described as single-tracked, double-tracked or multi-tracked according to the number of tracks. Main tracks (e.g. track 1 and track 2 in Figure 3-1) and siding tracks (e.g. track 3 and track 4) are defined (when the line and stations are designed). Tracks also can be 
configured with direction property, which is distinct from train direction, e.g. let the direction from station 01 to station 03 be up-direction, and conversely be downdirection, then a unidirectional track can be used in either up-direction or downdirection while a bidirectional track can be used in both directions.

Segment (arc): let a segment be a specified part of track between two adjacent precise points in the direction of train movement. Precise Point here is the point with precise pre-known location information usually can be a signal, a switch extremity, an ending, etc., corresponding to the interlocking definition.

This definition is different from that in literature (Törnquist and Persson, 2007) where 'each segment (include station) has a set of parallel tracks' or that in literature (Min et al., 2011) where 'a segment is the set of tracks connecting two adjacent stations'. A segment is the smallest cell of a track in this dissertation, i.e. a track consists of a complete set of segments fully bonding with it (both points), while a switch segment (e.g. segment a, in Figure 3-1) also connects double tracks.

Section: a section is a general name of all facilities in a predefined area. In Figure 3-1, a section can be either a station or the corridor between two stations, e.g. section 1, 3 and 5 represent stations, in due order composed of 5, 4, 3 tracks, while section 2 and 4 are the sections respectively connecting station 01 and 02 , station 02 and 03 , whose track numbers respectively are 2 and 1 . To be more detailed, section 2 is double-tracked and can be either unidirectional or bidirectional while section 4 is sing-tracked and in most cases it should be bidirectional. If in another railway line station 01 and station $\mathrm{X}$ are connected, then a section between them is also to be defined, distinguishing from section 2 .

As shown in Figure 3-1, a line consists of all stations and sections, tracks and signals, or all connected segments, etc., along the specific line geometry.

Blocking is the technique of safety control of the separation between trains by dividing the line into blocks. And there shall not be more than one train in each block at the same time. A block can either be a fixed block or a moving block.

Fixed block is a block in which the extremities of the block are fixed points with specific locations. The length of a fixed block is definite. The signalling allows a train to move from one block to the next, normally only when the block ahead is clear. Moving block is a block whose start point and length are defined by the characteristics of the train occupying the section of track. There are several types of moving block. Under the strictest definition, the minimum block length is from the rear most part of the occupying train to a point on the track where, if the train braked from its current speed, the front part of the train would be when the train came to a stand.

To be clear and definite, we note that only when fixed blocking technique is applied a segment and a block can be used to denote the domain of a same object, otherwise 
blocks changes with the blocking technique used. More specifically a segment generally has a fixed spatial character while a block has more sense in restraining a safe distance for sequential trains.

Route: a route can be represented as an ordered set of segments in order to complete a certain operation. According to the functions, a route can be receiving route (that may also be called arrival route, when a train arrives at station), departure route (when a train departs from the station), passing route (when a train directly passes through a station without stop) and shunting route (for a train to carry out shunting movement), etc.

Path: indicating train path, describing the sequence of all sections that an expected train has to transit from an original position (usually a start station) to its final destination (terminal station). In most cases, a train path may also contain the default routes at all stations and their scheduled times (e.g. arrival times, pass or departure times, etc.).

Timetable: it shows all train paths scheduled for a certain line or network, in the form of graph or table, where not only train set and their paths, but also the information of station set (in mileage order) and route sequences in stations, etc. are completely or partly available.

Generally, a basic timetable in railway is public and instructive in a long-term way, a daily timetable is informed one day or a few days in advance, and a real-time timetable in fact is partly the current train tracking paths or historic records, according to which the other part of short-term timetable is calculated in a short period time span. Rescheduling problem is mainly about the real-time timetable.

Conflict: a conflict at operational level is the situation that a train cannot exactly realise its plan due to any disturbance or interruption in railway network, such as wayside device failure, train fault, route unavailability or train delays, etc. Conflicts may exist at all stages, e.g. stage of strategic planning, tactical scheduling or real-time operation. What we focus on are those real-time conflicts occurring in daily traffic operation.

The models applied in this thesis tend to represent the important entities of the railway traffic based on their static information and dynamic collected data, rather than the functional design of each entity.

\subsection{Train movement model}

The model of train movement in this work has to be able to indicate a train independently, without accessing the kernel functions of train.

The collectable data of a train includes: 
- Static information: the name of train (or train number), the type, the length, empty loading weight and operational loading weight, static speed profiles, maximum speed, maximum acceleration and deceleration rates and other train control parameters;

- And the data has to be continually collected: head location, tail location, instantaneous speed, and the times of head entering a particular position or tail exiting from the position, etc.

Not all information is required in this model. To be distinct from each other, the train number is considered to be the key (both the identity and name) of a train, indicated as a superscript $i$ in $T^{i}, T^{i} \in T$. Here $T$ is the set of trains. Other properties of the train are implied as $T^{i}$ is mentioned. Those notations contained in the model are defined in Table 3-1.

Table 3-1 Symbol definitions of train movement model

\begin{tabular}{|c|c|c|}
\hline No. & Notation & Representation \\
\hline 1 & $T^{i}$ & Train $i, T^{i} \in T$ \\
\hline 2 & $T^{i}$ _len & Train length $[\mathrm{m}]$ \\
\hline 3 & $P^{i}$ & Train path of $T^{i}$ \\
\hline 4 & $P_{-}^{i} B(t)$ & Empirical segment blocking distribution of $P^{i}$ \\
\hline 5 & $P^{i}{ }_{-} V(x)$ & Empirical speed profile extended according to $P^{i}[\mathrm{~km} / \mathrm{h}]$ \\
\hline 6 & $g^{i} \_x h(t)$ & Head position of $T^{i}[\mathrm{~km}]$ \\
\hline 7 & $g^{i} x t(t)$ & Tail position of $T^{i}[\mathrm{~km}]$ \\
\hline 8 & $g^{i}{ }_{-} v(t)$ & Speed of train $[\mathrm{km} / \mathrm{h}]$ \\
\hline 9 & $g^{i}{ }_{-} X e o a(t)$ & Safety distance to the end of movement authority (MA) \\
\hline 10 & $g^{i} \operatorname{Vloa}(t)$ & The permitted speed at the end of authority $[\mathrm{km} / \mathrm{h}]$ \\
\hline 11 & $H\left(t, i_{1}, i_{2}\right)$ & Defined headway between succeeding trains $i_{1}, i_{2}$ \\
\hline
\end{tabular}

We assume the train length $T^{i}$ len is smaller than $900 \mathrm{~m}$ if it is a passenger train, or is not larger than $1500 \mathrm{~m}$ of a freight train. The defined headway between succeeding trains is not less than the minimum technical headway. In this model the minimum headway distance or minimum headway time is pre-known, or at least is acquirable from the other given data.

Each train holds a target path when it is scheduled, i.e. the sequence of all sections that the expected train has to transit from a start station to its final destination during a certain time period. Thus, the general path $P^{i}$ can be obtained from the graphic timespace schedule. Since the model considered in this work is data-based, $P^{i} B(t)$ and $P^{i}{ }_{-} V(x)$ are referred to describe the segment transfer and speed profile of train $i$, respectively. $P^{i} B(t)$ and $P_{-}^{i} V(x)$ are derivatives of train path $P^{i}$, and they can be produced off-line.

Actually factors obtained via dynamic collection are the head and tail positions, as well as the speed of train. When the integrity of train is not the issue to take account in 
the model, the tail position of train can be derived from the head position and the length of train. They will be mainly supervised and analysed. The end of authority $g^{i} \_$Xeoa $(t)$ and the target speed $g^{i}$ _Vloa $(t)$ at this end, indicating the permitted braking distance and speed, are defined to represent the key information of a movement authority.

\subsubsection{Static derivatives of train path}

Blocking segment estimation $P^{i} B(t)$ tracks the occupied segments and transfer time while a train is routinely operated to realise its target path $P^{i}$ and the trackside systems are all working regularly. Figure 3-2 illustrates the derivative $P^{i}{ }_{-} B(t)$, where the colour of $P^{i}{ }_{-} B(t)$ still reflects the speed changing at different segments. It should be noted that the segment time estimation is directly dependent on the physical definition of segments, rather than the blocking techniques.

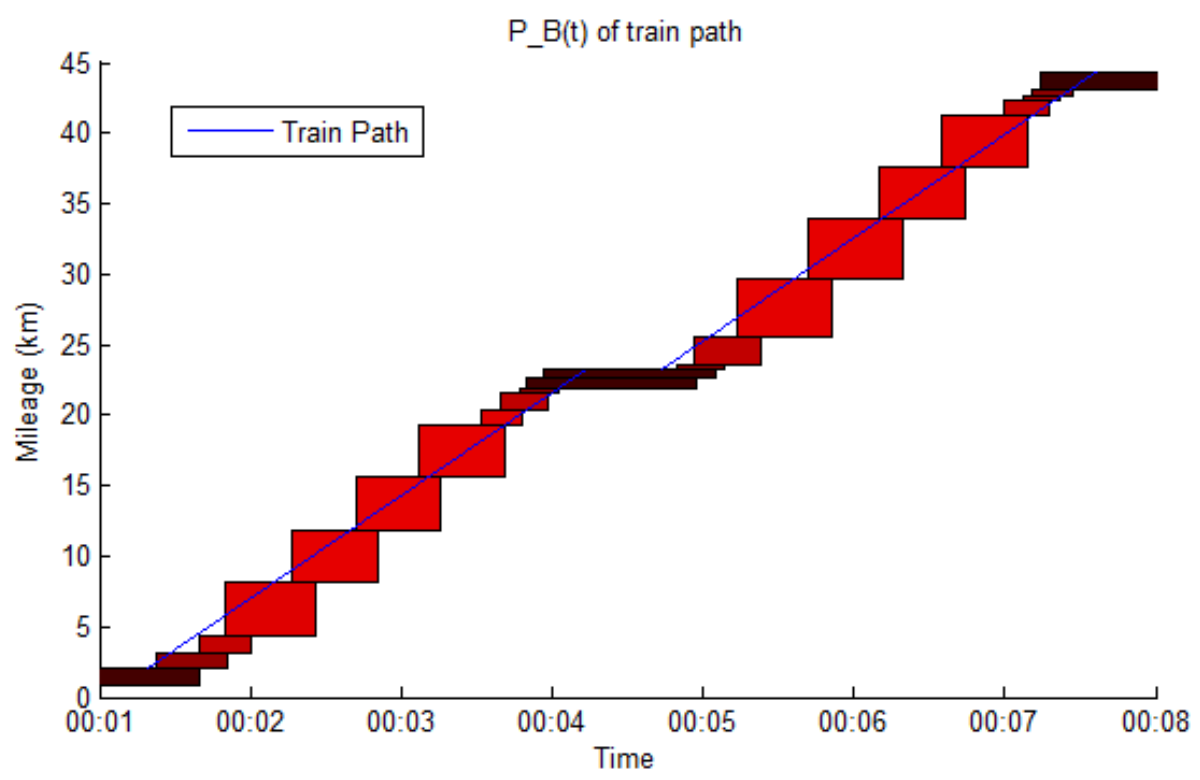

Figure 3-2 An illustration of blocking segment $P^{i}{ }_{-} B(t)$ according to $P^{i}$

Static speed restrictions (SSR) are mainly generated by considering the factors of line geometry, the train characteristics, the signalling, the application level of system, and the mode of the on-board equipment, etc. There are a number of categories of static speed restrictions, and these categories shall be independent on each other. The speed and distance monitoring assures that the train remains within the given speed and distance limits. So we can obtain the empirical speed profile $P^{i}{ }_{-} V(x)$ according to the historical data collection of train $i$, as it is shown in Figure 3-3, where the most restrictive speed profile (MSRP) represents the comprehensive (most restrictive) result of all categories of static speed restrictions that the train shall obey on a given piece of track (ERA et al., 2014).

The most restrictive speed profile and the empirical speed profile vary from train type to train type. And as the most restrictive speed profile shall be recalculated when any of 
the restrictive elements is changed, the empirical speed profile has to be re-collected as well.

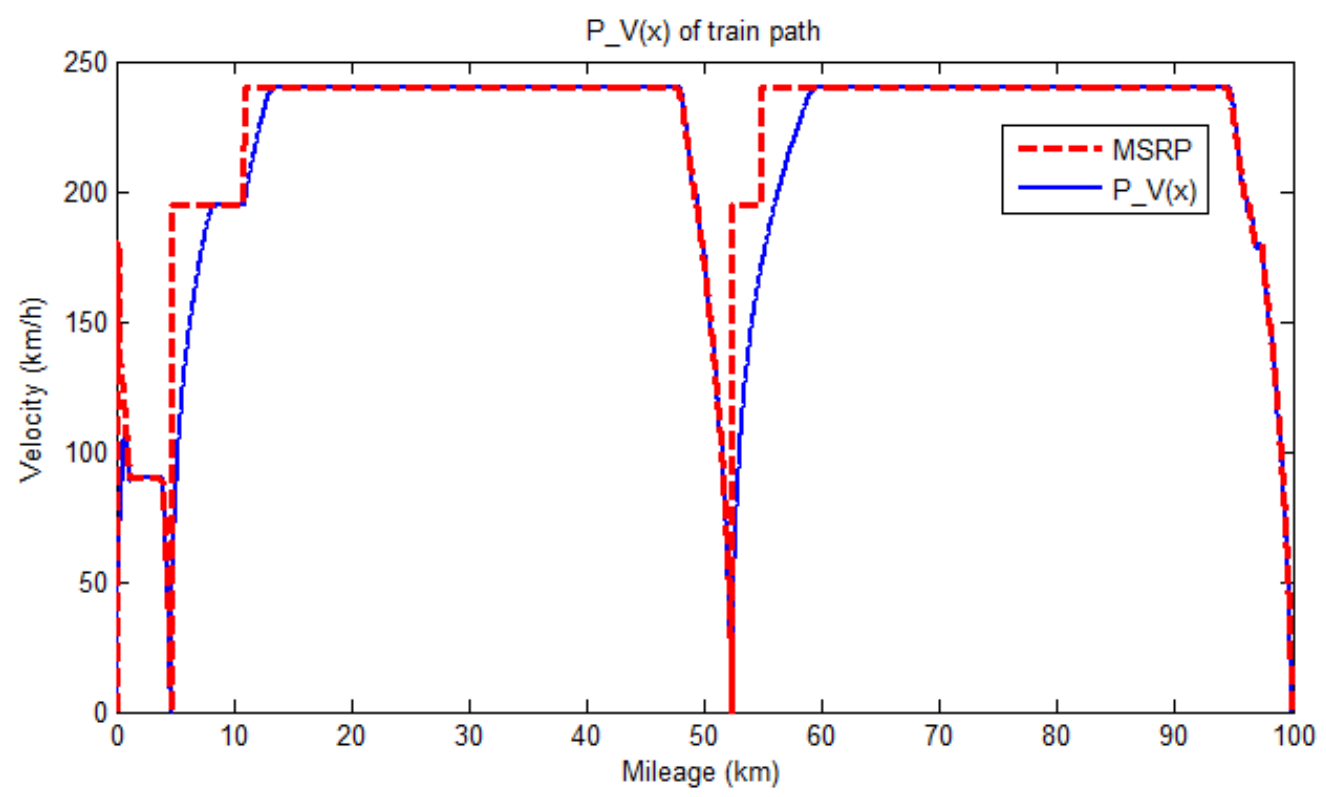

Figure 3-3 An illustration of empirical speed profile $P^{i}{ }_{-} V(x)$ according to $P^{i}$ and the most restrictive speed profile

\subsubsection{Dynamic train data}

The behaviour of dynamic train movement can be expressed by the following characteristics:

(a) The collected data in dynamic procedure, including the positions $g^{i} \_h(t)$, $g^{i}{ }_{-} x t(t)$, the speed of train $g^{i}{ }_{-} v(t)$, the safety distance to the end of authority $g^{i}{ }_{-} X e o a(t)$ and the maximum speed permitted $g^{i}$ Vloa $(t)$, as well the received track description.

(b) The predictive positons of train in a short period of time based on the most restrictive value of the permitted speed and the collected data.

\subsection{Infrastructure model}

The data-based model of infrastructure does not consider the control logic as an interlocking, thus it mainly contains the data of track characteristics, which is the base of track description.

Track description is the information which describes the characteristics of the track in order to supervise a train movement: as a minimum, the distance of the movement authority, static speed profile and gradient profile; and optionally, it can contain axle load profile, track conditions, route suitability data, areas where shunting is permitted (ERA et al., 2014).

Parameters and variables of the infrastructure model are defined in Table 3-2. 
Table 3-2 Symbol definitions of infrastructure model

\begin{tabular}{|c|c|c|}
\hline No. & Notation & Representation \\
\hline 1 & $S_{j}$ & Section $j, S_{j} \in S . S$ is the set of sections (stations are included). \\
\hline 2 & $S_{j-}$ type & $S_{j-}$ type $\in\{0,1\}$, it values 1 when $S_{j}$ is a station, otherwise 0 . \\
\hline 3 & $L_{j, s}$ & $\begin{array}{l}\text { Segment } s \text { in section } j, L_{j, s} \in L_{j} . L_{j} \text { is the segment set composing } \\
\text { section } j, L_{j} \in L . L \text { is the complete segment set of a line or a } \\
\text { network. } \\
L_{j, s} \text { has a code to express its most important properties, see in Figure } \\
3-4 \text {. }\end{array}$ \\
\hline 4 & $o_{j, s}(t)$ & $\begin{array}{l}\text { The state of segment, } o_{j, s}(t) \in\{0,1\} \text {. It values } 1 \text { when the segment } s \\
\text { is occupied, otherwise } 0 \text {. }\end{array}$ \\
\hline 5 & $E_{q}$ & $\begin{array}{l}\text { Station } q, E_{q} \in E . E \text { is the set of stations. } \\
\text { Each station has the information of all its entries, platforms and } \\
\text { routes. Neighbour sections physically connect by the entries they } \\
\text { share. }\end{array}$ \\
\hline 6 & $m_{q, e}(e, t)$ & $\begin{array}{l}\text { The message from entry } e, e \in E_{q \_} \text {entries. This message provides } \\
\text { the detected time of train entering a station or train exiting from a } \\
\text { station. }\end{array}$ \\
\hline 7 & $R_{q, r}$ & $\begin{array}{l}\text { Route } r \text { in } E_{q}, R_{q, r} \in E_{q-} \text { routes. Each route has a sequence of } \\
\text { segments and other corresponding parameters, see in Figure } 3-5 \text {. }\end{array}$ \\
\hline 8 & $r_{q, r}(t)$ & $\begin{array}{l}\text { The state of route, } r_{q, r}(t) \in\{0,1\} \text {. It values } 1 \text { when the route } r \text { is } \\
\text { reserved or occupied, otherwise } 0 \text {. }\end{array}$ \\
\hline 9 & $k_{q, k}(t)$ & $\begin{array}{l}\text { The state of (platform) track } k \text { in station } q, k_{q, k}(t) \in\{0,1\} \text {. It values } \\
1 \text { when track } k \text { is in use, otherwise it values } 0 \text {. }\end{array}$ \\
\hline
\end{tabular}

In this model, the indexes of all objects are defined in subscripts.

Concretely, at a line level (or a network level) sections is indexed by $j$. When a section is station, it is also denoted by a station entity, indexed by $q$, with defined entries, by which the neighbour sections physically connect. At a regional level, the segment in a section is indexed by $s$, and the route is indexed by $r$. A segment in this model is the very basic piece of the track whose information includes spatial location (defined by the precise positions of its extremities), linking relationships and its speed restrictions, etc., see in Figure 3-4. The information implied in a route is illustrated in Figure 3-5.

If we define routes for all sections, i.e. the open section between stations, whose $S_{j}$ type values 0 , also considers its route characteristics, the route notation can be extended as $R_{j, r}$ and the real-time state of route as $r_{j, r}(t)$. This extension is used in the state transition method of conflict detection. 


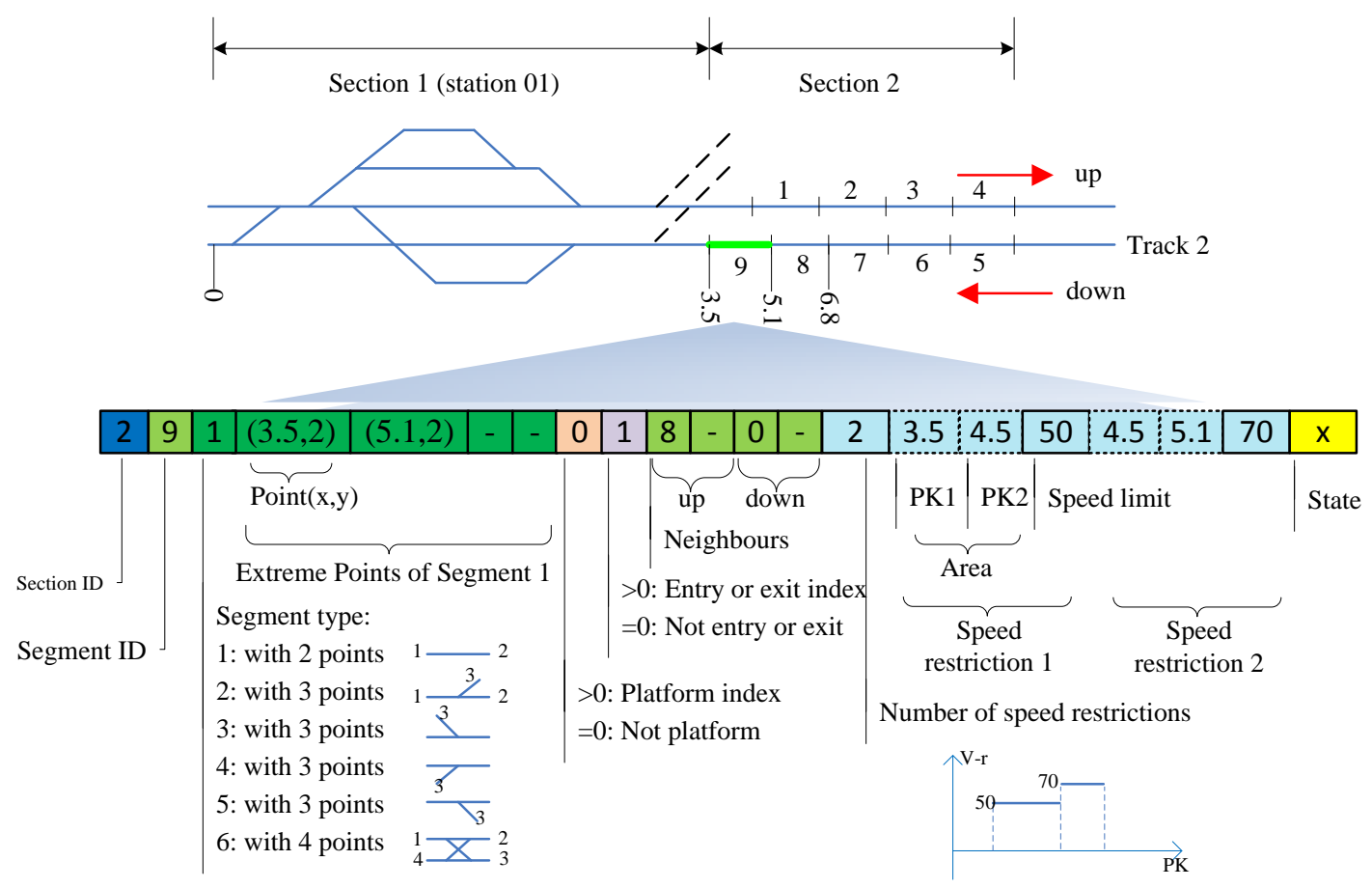

Figure 3-4 Segment description with static properties and real-time state $\left(L_{j, s}+o_{j, s}\right)$

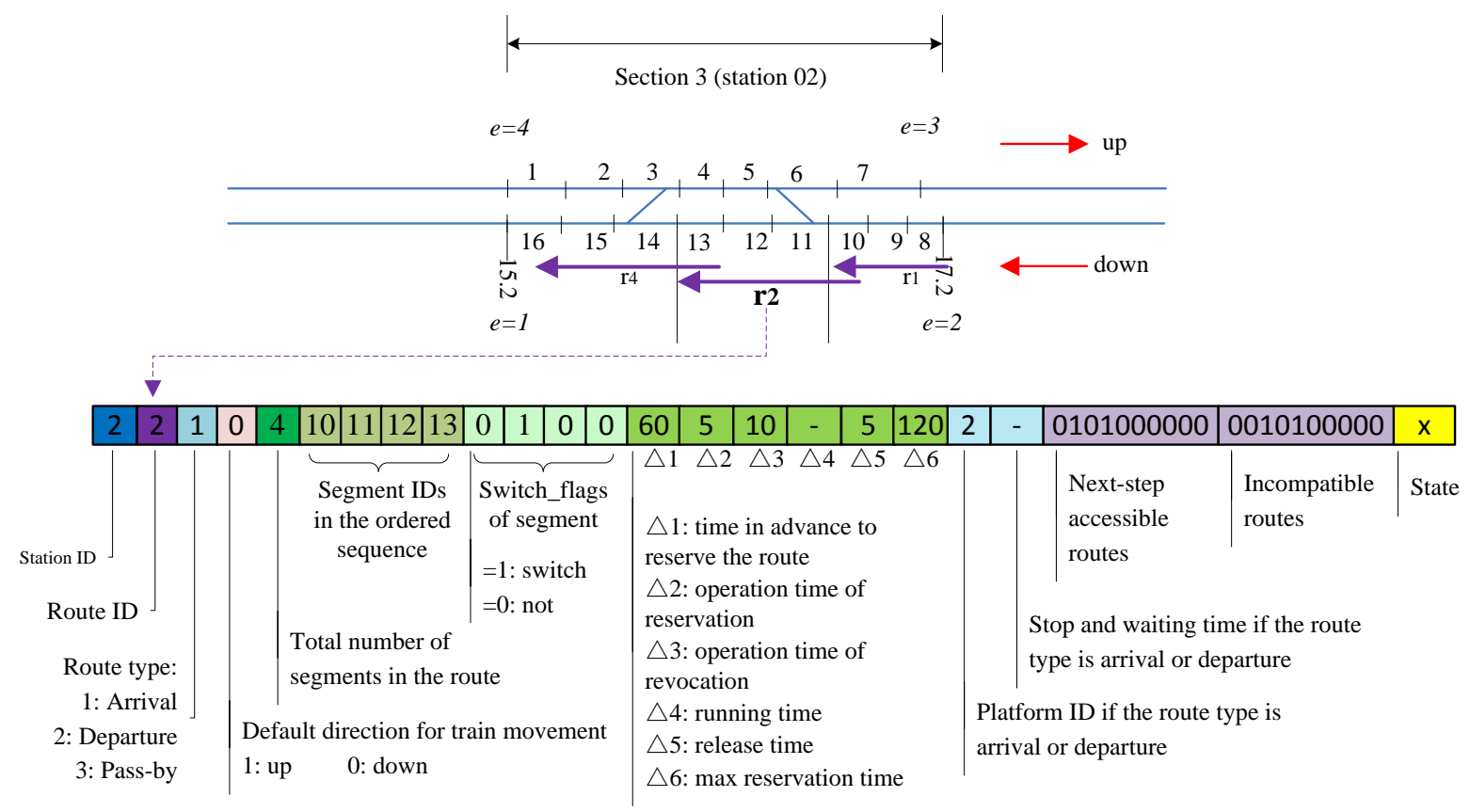

Figure 3-5 Route description with static properties and real-time state $\left(R_{q, r}+r_{q, r}\right)$ 

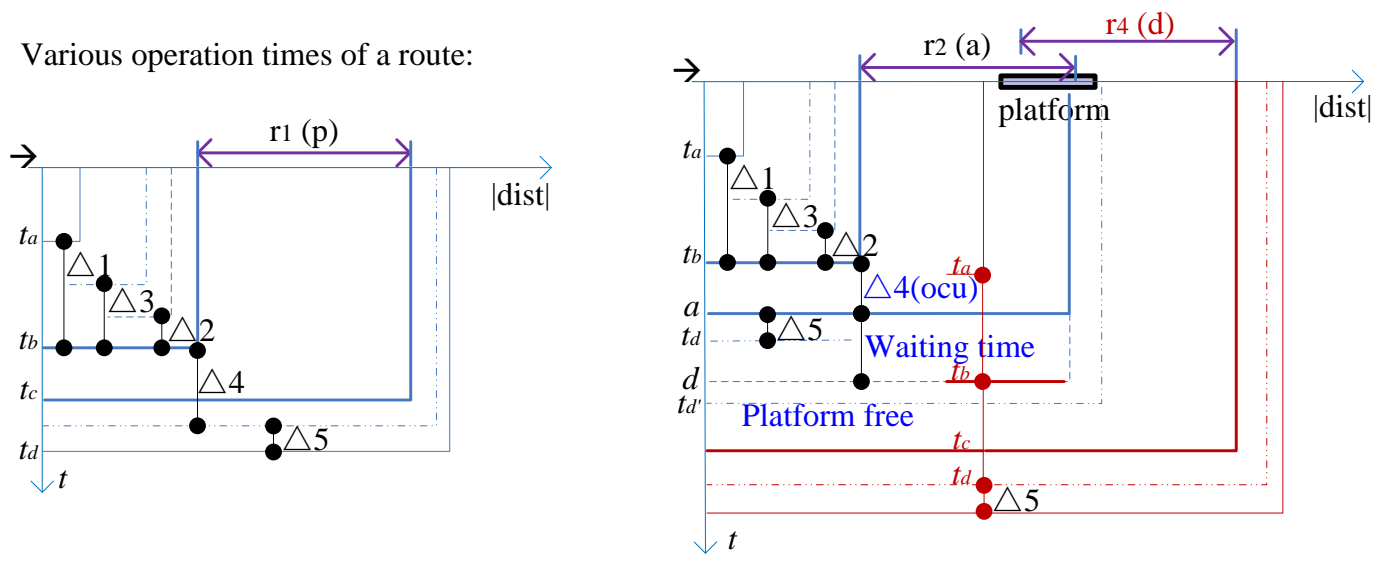

Figure 3-6 An illustration of defined times in route description

\subsubsection{Static information}

The majority of the information shown in Figure 3-4 and Figure 3-5 is static. It contains the basic properties of a segment and a route. Meanwhile, it implies the structural and restrictive relationships in the embedded infrastructure environment.

The ID, type, extremity points, entry or platform check, speed restrictions and neighbours are defined in the segment, where the type of segment determines the number of attached extremity points. An extremity point actually is the position information described in two dimensions $(x, y): x$ is the mileage in $\mathrm{km}$ and $y$ is the track code. When the segment type values $2,3,4$, or 5 , the track code $y$ of the third point is not integer. The speed limitation function of the segment is piecewise described, which is in accordance with the static speed profile mentioned in Section 3.2.1. The neighbour segments of segment $s$ respectively in up-direction and down-direction are referred by their IDs. Supposing that there are $N_{s}$ segments in the section, we can obtain a $\left(N_{s} \times N_{S}\right)$ adjacency matrix of segment, where all the neighbours are ones and others are zeros.

Similarly, the ID, type and the segment constitution of the route are first defined. Besides, the default direction of the route is given. The segment sequence appears according to the default direction. And if there are switches on the route, they are marked in the same order of segment sequence. Operation times, including time on reservation/revocation/release of the route and theoretical running time, etc. are important parameters in investigating the route capacity and route control, see Figure 3-5 and Figure 3-6. The time in advance to reserve/revoke a route indicates that only in a proper time range, the operation of reservation/revocation is allowed. But a maximum reservation time is also employed to prevent the route being long time reserved but not actually used. The route can be cancelled automatically after a maximum reservation time. If it is an arrival route or a departure route, the information of platform and waiting time is also described. Last, the next-step accessible routes and the incompatible routes are referred. Supposing that the total number of routes in the section is $N_{r}$, correspondingly, two $\left(N_{r} \times N_{r}\right)$ matrices (one-step accessibility matrix and 
incompatibility matrix) of route are set up. These matrices model the connecting relationships and interlocking constraints of the railway network.

\subsubsection{Dynamic state transition}

According to the definitions in Table 3-2, the following variables are time dependent: event message $m_{q, e}(e, t)$, segment state $o_{j, s}(t)$, route state $r_{q, r}(t)$ and platform usage state $k_{q, k}(t)$. Especially, as Figure 3-7 shows, the segment state $o_{j, s}(t)$ combining with its related properties basically leads the changes of other state variables.

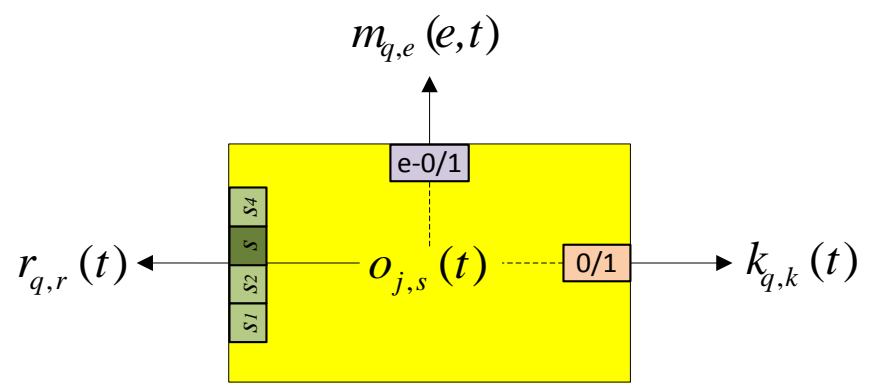

Figure 3-7 Fundamental role of segment state

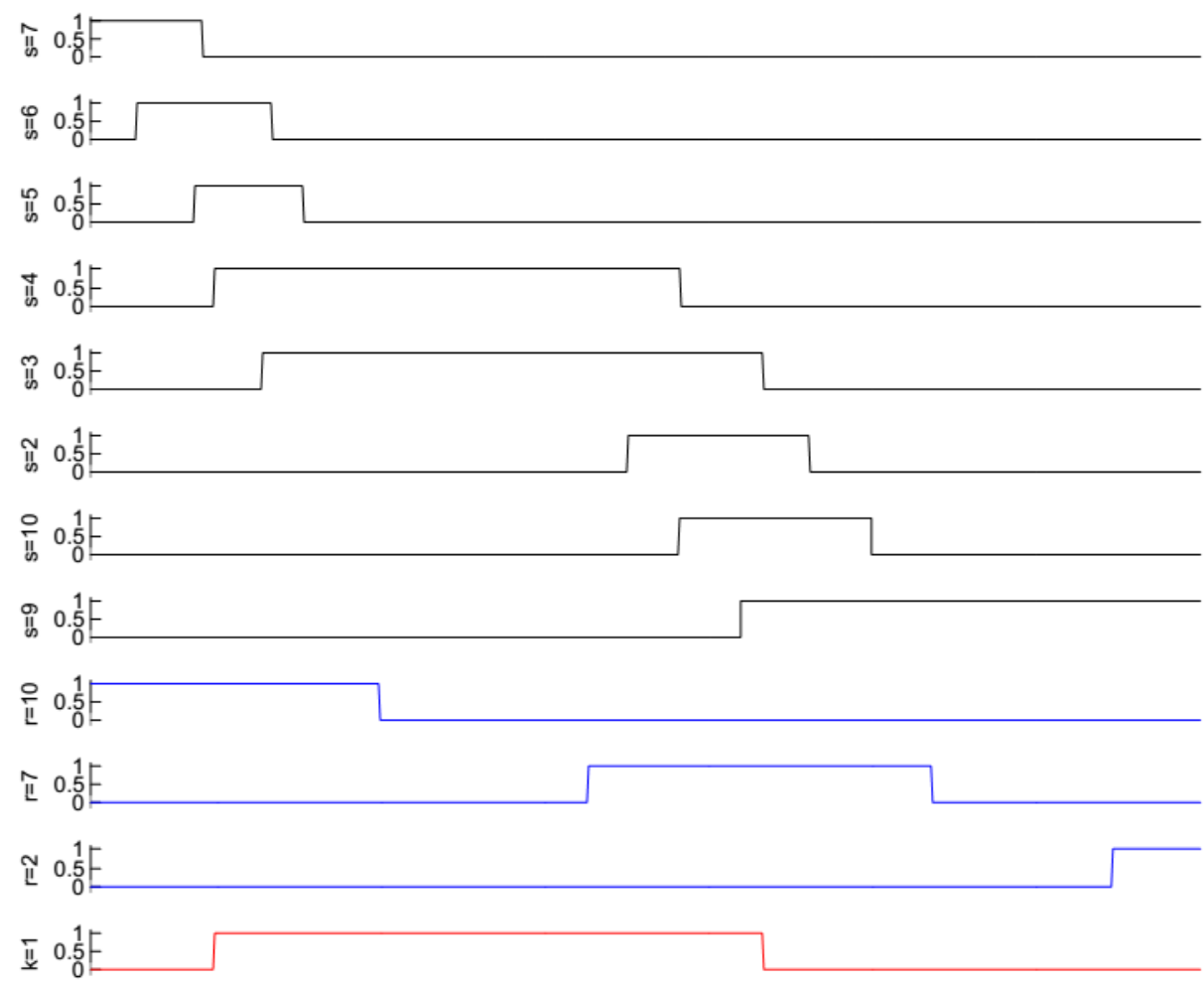

Figure 3-8 State diagram of state variables $\left(o_{j, s}(t), r_{q, r}(t), k_{q, k}(t)\right)$

The time-varying value of each binary state variable in this model can be recorded via its corresponding state diagram, as the illustration shown in Figure 3-8. Furthermore, the state diagram implies a series of time information, such as train entering (exiting) time, arrival time, average state transition time, operation time to complete certain 
operation at a station, etc. The state variables of the same type further compose the dynamic state vector of this variable type, for example, segment state vector of all $o_{j, s}(t)$ and route vector of all $r_{q, r}(t)$, which will be explained in detail in Chapter 4 .

\subsection{Timetable representation}

The timetable usually plays an important role in organising the resources of the railway system. It can be a basic timetable or an updated timetable in real-time traffic control.

\subsubsection{Basic timetable}

A basic timetable is the primary reference and target for trains and stations to carry out their tasks. A basic timetable is well predesigned off-line, and it is conflict-free. Assume that there are $N_{t r}$ trains and $N_{s t}$ stations scheduled in the timetable. The contents defined in a basic timetable and the related records in traffic management are shown in Table 3-3.

Table 3-3 Symbol definitions about basic timetable and related records

\begin{tabular}{|c|c|c|}
\hline No. & Notation & Representation \\
\hline 1 & $A_{q}^{i} \in A$ & $\begin{array}{l}\text { Arrival time for } T^{i} \text { at station } E_{q} . A \text { is the set of arrival times at } \\
\text { scheduled stations. } \\
\text { If } A_{q}^{i} \in A^{i}, A^{i} \in A \text { is further defined, } A^{i} \text { is the arrival time set for } \\
T^{i} \text { to make a single travel. }\end{array}$ \\
\hline 2 & $D_{q}^{i} \in D$ & $\begin{array}{l}\text { Departure time for } T^{i} \text { from station } E_{q} . D \text { is the set of departure } \\
\text { times at scheduled stations. }\end{array}$ \\
\hline 3 & $\tau_{j}^{i} \in \tau$ & $\begin{array}{l}\tau \text { is an extended set of all entering times indexed by sections (not } \\
\text { only stations). We define } \tau_{j}^{i}>0 \text {. }\end{array}$ \\
\hline 4 & $W_{j}^{i} \in W$ & $\begin{array}{l}W \text { is the set of minimum wait times. } W_{j}^{i} \text { is positive at a scheduled } \\
\text { stop station and is } 0 \text { when } S_{j} \text { is not scheduled to stop for } T^{i} \text {. }\end{array}$ \\
\hline 5 & $K_{q}^{i} \in K^{i}$ & Optional, $K_{q}^{i}$ is the set of track (platform) at station $q$ for $T^{i}$. \\
\hline 6 & $a_{q}^{i}$ & Recorded arrival time of $T^{i}$ at station $q$. \\
\hline 7 & $d_{q}^{i}$ & Recorded departure time of $T^{i}$ at station $q$. \\
\hline 8 & $t_{j}^{i}$ & Recorded head-entering time of $T^{i}$ at section $j$. \\
\hline 9 & $k_{q}^{i}$ & Actual platform allocated to $T^{i}$ at station $q$. \\
\hline 10 & $z_{q}^{i}$ & Recorded delay of $T^{i}$ at station $q$. \\
\hline 11 & $\vec{z}_{j}^{i}$ & $\begin{array}{l}\text { Recorded time difference when } T^{i} \text { is entering section } j \text {, referring } \\
\text { to } \tau_{j}^{i} \text {. }\end{array}$ \\
\hline
\end{tabular}

Minimum stop time at each station is also a parameter that has been used in timetabling. In the timetable, the difference between departure time and arrival time is actually larger than the value of corresponding minimum stop time, i.e. $D_{q}^{i}-A_{q}^{i}>W_{j}^{i}$ where the station $E_{q}$ is indexed $j$ in sections. In this model, we consider $D_{q}^{i}=A_{q}^{i}$ when 
the train is scheduled to directly pass through the station $q$. The train path $P^{i}$ may contain the information of $A^{i}, D^{i}$ and $K^{i}$.

The set of actual records $(6,7,8$ and 9) of train arrival time, departure time, entering time and the used platform are obtained in traffic supervision, and compared to the planned ones. The times defined in this section are illustrated in Figure 3-9.
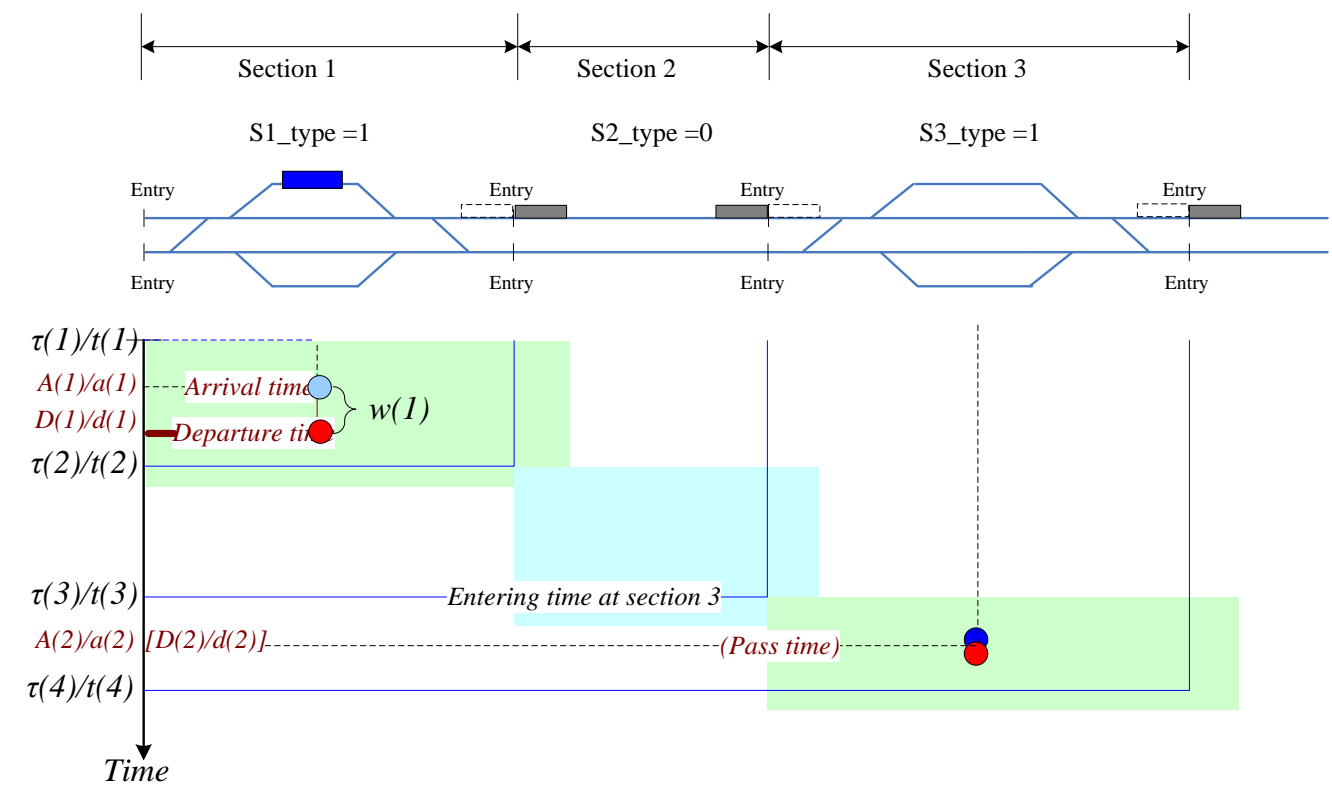

Figure 3-9 An illustration of arrival time, departure time and entering time

Recorded train delay $z_{q}^{i}$ at station is obtained by

$$
z_{q}^{i}=\left\{\begin{array}{c}
a_{q}^{i}-A_{q}^{i}, \text { when } a_{q}^{i}>A_{q}^{i} \\
0, \text { otherwise }
\end{array}\right.
$$

Train delay is always detected and as an evaluation indicator in traffic control. To minimise the total delay or other delay related parameters is also a main objective of traffic management and timetable optimisation.

Recorded entering time difference $\vec{z}_{j}^{i}$ is estimated by (3-2), which can also be a negative value.

$$
\vec{z}_{j}^{i}=t_{j}^{i}-\tau_{j}^{i}
$$

\subsubsection{Rescheduled timetable}

As implied, once the train delay (major delay) is recorded, the traffic controllers may have to update the timetable according to the actual situation. In this case, the timetable can be locally or globally rescheduled if necessary. The contents taken account in timetable rescheduling are defined in Table 3-4. 
Table 3-4 Symbol definitions about rescheduled timetable

\begin{tabular}{lll}
\hline No. & Notation & Representation \\
\hline 1 & $\widetilde{A_{q}^{l}} \in \tilde{A}$ & $\begin{array}{l}\text { Rescheduled arrival time for } T^{i} \text { at station } E_{q} . \tilde{A} \text { is the set of arrival } \\
\text { times after rescheduling. }\end{array}$ \\
2 & $\widetilde{D_{q}^{l}} \in \widetilde{D}$ & $\begin{array}{l}\text { Rescheduled departure time for } T^{i} \text { from station } E_{q} . \widetilde{D} \text { is the set of } \\
\text { departure times after rescheduling. }\end{array}$ \\
3 & $\widetilde{K_{q}^{l}} \in \widetilde{K^{l}}$ & $\begin{array}{l}\text { Optional, the re-allocated track (platform) to stop at each station } \\
\text { for } T^{i} .\end{array}$ \\
4 & $Q_{q_{1}, q_{2}}^{i} \in Q$ & $\begin{array}{l}\text { Minimum running time for a train (a type of trains) to depart from } \\
\text { station } q_{1} \text { and arrive at station } q_{2} .\end{array}$ \\
\hline
\end{tabular}

All arrival or departure times are not necessary to be rescheduled.

Apart from the minimum stop times mentioned in Table 3-3, minimum running times for a train type are considered in rescheduling. The rescheduled times are subject to these minimum time restrictions.

$$
\begin{aligned}
& \widetilde{D_{q}^{l}}-\widetilde{A_{q}^{l}} \geq W_{q}^{i}, \quad W_{q}^{i} \in W \\
& \widetilde{A_{q_{2}}^{\tau}}-\widetilde{D_{q_{1}}^{l}} \geq Q_{q_{1}, q_{2}}^{i}, \quad q_{2} \text { is the next station for train } i \text { after station } q_{1}
\end{aligned}
$$

In rescheduling, the exact train length may not be considered directly in calculation of the running time. Instead, an estimated maximum clearing time will be used.

\subsubsection{Graphic representation}

On the base of the existing 2-dimensional time-space schedule graph, we add a third dimension of track.

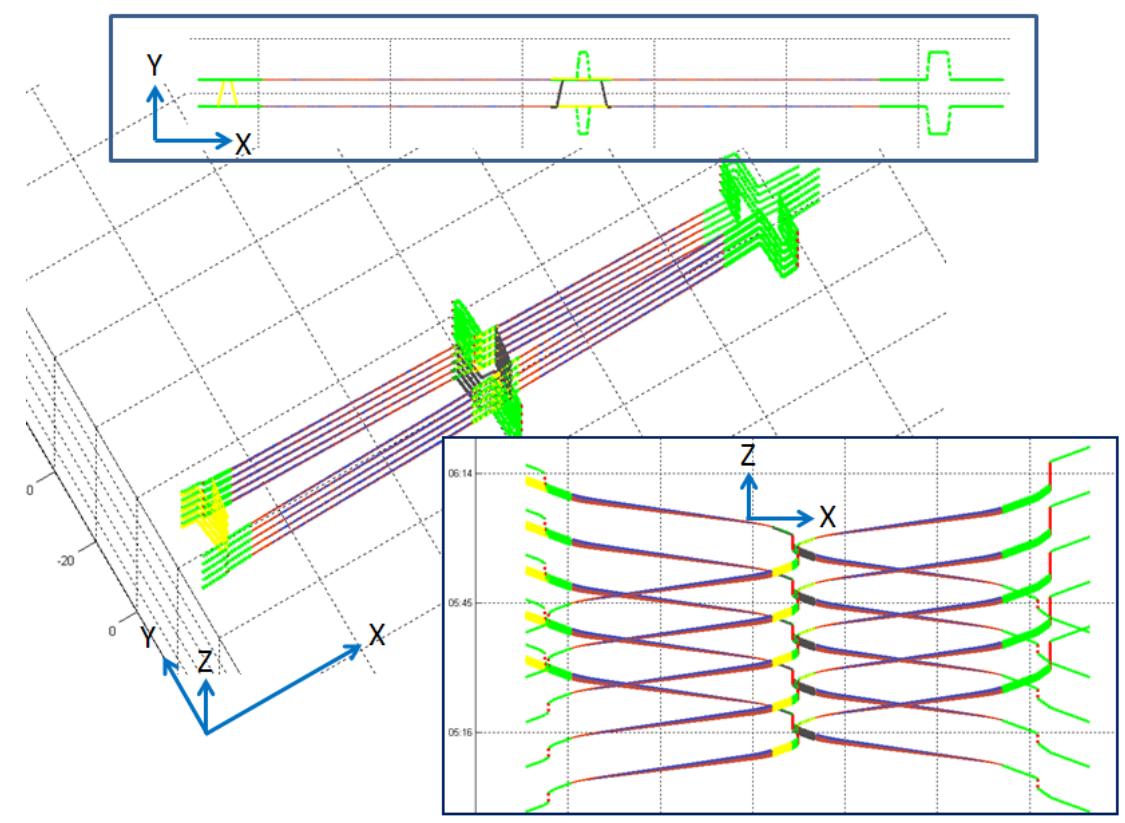

Figure 3-10 A time-space schedule in 3 dimensions 
In the illustration (see in Figure 3-10), $\mathrm{X}$ dimension is the mileage of the line and $\mathrm{Y}$ dimension is a reflection of the relative (paralleled) position of track, and $\mathrm{Z}$ dimension is time. To be meaningful in practical problem, the two spatial dimensions $\mathrm{X}$ and $\mathrm{Y}$ are always restricted in joint definition. That is, if we have known all valid field of the actual railway (tracks) topology, denoted by $N(X, Y)$, then any location $(x, y)$ on the network belongs to this particular domain, i.e. $(x, y) \in N(X, Y)$.

In a 3-dimensional representation, we also can get the traditional 2-dimensional network plane $(\mathrm{X}-\mathrm{Y})$ view and distance-time $(\mathrm{X}-\mathrm{Z})$ view.

\subsection{Interaction of partial models}

The models presented in the previous sections are not independent. Figure 3-11 shows the great bonds of variable references that connect the partial models of timetable, train and railway network.

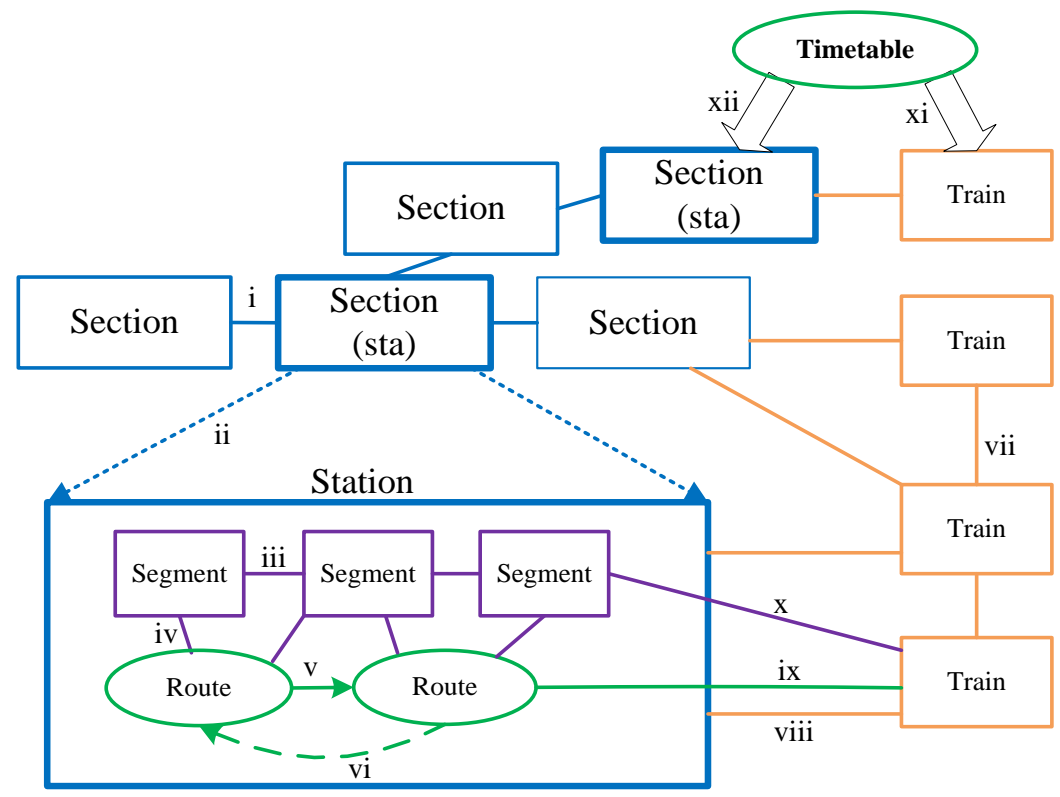

Figure 3-11 Variable references among the models

Concrete connections in Figure 3-11 are explained as follows.

i) Connection between two sections

ii) Binary judgement of whether a section is a station

iii) Adjacency segments

iv) A segment is (or is not) part of a certain route

v) Two routes can be assigned in succession

vi) (In-)Compatible routes

vii) Order and headway relationship between trains

viii) Train uses the resource of a section, with the target arrival and departure times at a station

ix) A proper route is (or is not) assigned to a train 
$\mathrm{x}$ ) A particular segment is (or is not) occupied by a train

xi) All trains aim to follow the train paths extracted from the timetable

xii) Each section has a scheduled train sequence as well according to the timetable

Owning to the internationally standardised characteristics of ERTMS/ETCS, the integrated model of traffic may also benefit from the advantages of ERTMS/ETCS. In ERTMS/ETCS system a train as well as the trackside equipment are respectively specified to act and react in a uniform way. Yet the specifications may be distinct when they are defined for each particular level of ERTMS/ETCS. It enables the modelling of traffic in a relatively unified form. On the other hand, the implemented functions for ERTMS/ETCS trackside subsystem are defined according to the characteristics of the specific lines and the related operational needs. It provides the access for the nonstandardised conflict detection and resolution (CD and CR) to employ functions into ERTMS/ETCS via trackside subsystem and external systems.

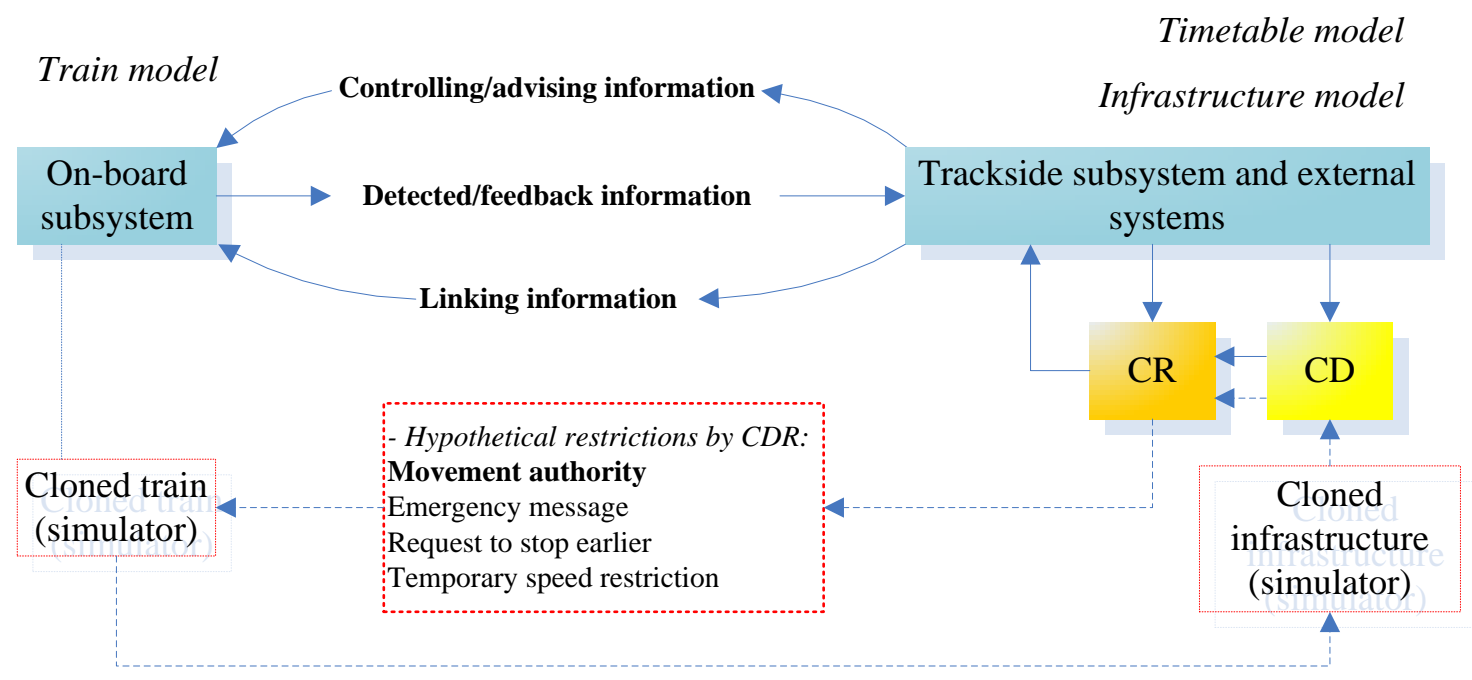

Figure 3-12 Conflict detection and resolution frame based on a typical ERTMS/ETCS system

At the developing stage, a framework of conflict detection and resolution integrating with the ERTMS/ETCS system is shown in Figure 3-12. Firstly, the CD module starts with the real-time collected data from each partial model. Meanwhile, we assume that for each train there is a virtual clone (parallel simulator) with equivalent data and functions. A train is real-time controlled, whilst its virtual clone is allowed to simulate in a future period of time based on the real-time situation from its entity train. That is, hypotheses and equivocal tests that have not been accepted in an entity train may exist in the virtual train. Similarly, we assume that for each part of infrastructure (e.g. for each section) there is a virtual clone. The simulation of cloned trains at the same time results in the state changes of the cloned infrastructure, and the latter provides the testing data for further conflict detection and resolution. Predictive information and feasible solutions tested by the cloned models are sent back to the trackside subsystem. 
The trackside subsystem may decide to accept/reject the proposal or ignore the information.

\subsection{Conflict signs}

There are usually some signs presaging corresponding potential conflicts. These signs can be what we consider the disturbances or interruptions.

\subsubsection{Signs in train movement}

Actual information of train movement (next safety distance, current position and speed) is sent to the traffic control centre at appropriate frequency, thus the positions $g^{i}{ }_{-} x h(t), g^{i}{ }_{-} x t(t)$ (with a confidence interval) and the speed of train $g^{i} \_v(t)$ are dynamically updated. The collected data of each train can be plotted in a distance-speed diagram and real-time updated. We name all these distance-speed diagrams $G \_V(x)$ profiles. At the same time, the empirical speed profile $P^{i}{ }_{-} V(x)$ is available, providing a reference of $G^{i}{ }_{-} V(x)$ profile. For each individual train $i$, it is considered a sign of conflict if any of the following occurs.

(1) The actual $G_{-}^{i} V(x)$ profile is significantly different from the empirical speed profile $P^{i}{ }_{-}(x)$;

$$
\left|G^{i}{ }_{-} V(x)-P_{-}^{i} V(x)\right| \geq \Delta v
$$

Where $\Delta v$ is a predefined threshold value to judge a difference is significant or not, $\Delta v>0$.

(2) The absolute distance from the rear end of a foregoing train $i_{1}$ to the front end of the succeeding train $i$ is not satisfying the defined headway restriction.

$$
\left|g^{i_{1} \_} x t(t)-g^{i} \_x h(t)\right|<H\left(t, i_{1}, i\right)
$$

\subsubsection{Signs in infrastructure state}

The real-time state of each object in infrastructure is supervised as well. The control centre has the knowledge on safety states of infrastructure. That is, when a train occupies (is going to occupy) some portion of the track, the control centre (controller or local dispatcher) can judge the validity of the state.

In the infrastructure model, as shown in Figure 3-8, segment states, route states and platform usage states are tracked. For a concrete section, if all states of the same type are evaluated as an integral group (represented as a state vector), each state vector can partly or completely represent the infrastructure state. Thus, abnormal states of any type of state vector will be firstly detected, and the possibility of conflict will be further estimated. The states are considered to be abnormal for any of the following reasons. 
(1) A new segment state (vector) that is not stored in the segment state domain table which lists all safety segment states;

(2) A new route state (vector) that is not stored in the route state domain table;

(3) A route state (vector) that indicates the coexistence of incompatible routes;

(4) A new platform usage state (vector) that is not in accordance with the predefined distribution (if any);

(5) Any type of state (vector) that maintains the same state for an irregular period of time (much shorter or much longer than its regular operational time).

\subsubsection{Signs in time records}

The recorded times and the scheduled times in timetable are always compared at stations. Delay detection is a common means to obtain the final effect of traffic control. In other words, it is considered that the traffic is disturbed if obvious delay is detected.

So for train $i$, another typical sign for conflict checking is

$$
z_{q}^{i} \geq \Delta z
$$

$\Delta z$ is a predefined threshold value to judge whether a delay is to be ignored, which can be defined according to the concrete line characteristics. If the timetable is rescheduled and conflict-free, then the alternative sign is referred to

$$
\tilde{z}_{q}^{i} \geq \Delta z
$$

where $\tilde{z}_{q}^{i}=\max \left\{a_{q}^{i}-\widetilde{A_{q}^{\imath}}, 0\right\}$.

Besides, if the actual platform allocated to train $i$ differs from any of the scheduled platforms (the default and the alternative):

$$
k_{q}^{i} \notin K_{q}^{i}
$$

Taking the time records of more trains into account, the headway time (interval) between the succeeding trains, which is defined as the time between two trains passing the same location on a railway line at a defined speed profile, is observable. If the recorded headway time starts to grow and is obviously larger than the planned interval, denoted as $I\left(x, i_{1}, i_{2}\right)$, the situation has to be checked. For example,

$$
t_{j}^{i_{2}}-t_{j}^{i_{1}}>I\left(x, i_{1}, i_{2}\right)+\Delta I
$$

$\Delta I$ is a predefined threshold value to judge whether the actual interval is obviously different from the planned one. This sign of conflict checking is often applied when the timetable is periodic scheduled. 


\section{Chapter 4}

\section{Conflict detection}

Train trajectory approach and infrastructure state transition approach are presented for traffic prediction and conflict detection. 


\section{Chapter 4 Conflict detection}

Signs of conflicts are not sufficient conditions for conflicts, but the triggers for calling conflict detection procedure and preventing any potential conflicts. In this chapter, it is introduced how a sign is judged to be the prophase of a potential conflict or is acquitted of a real conflict. Figure 4-1 presents a general structure of the detecting methodology. In this structure two parallel approaches are contained: train trajectory tracing and infrastructure state transition.

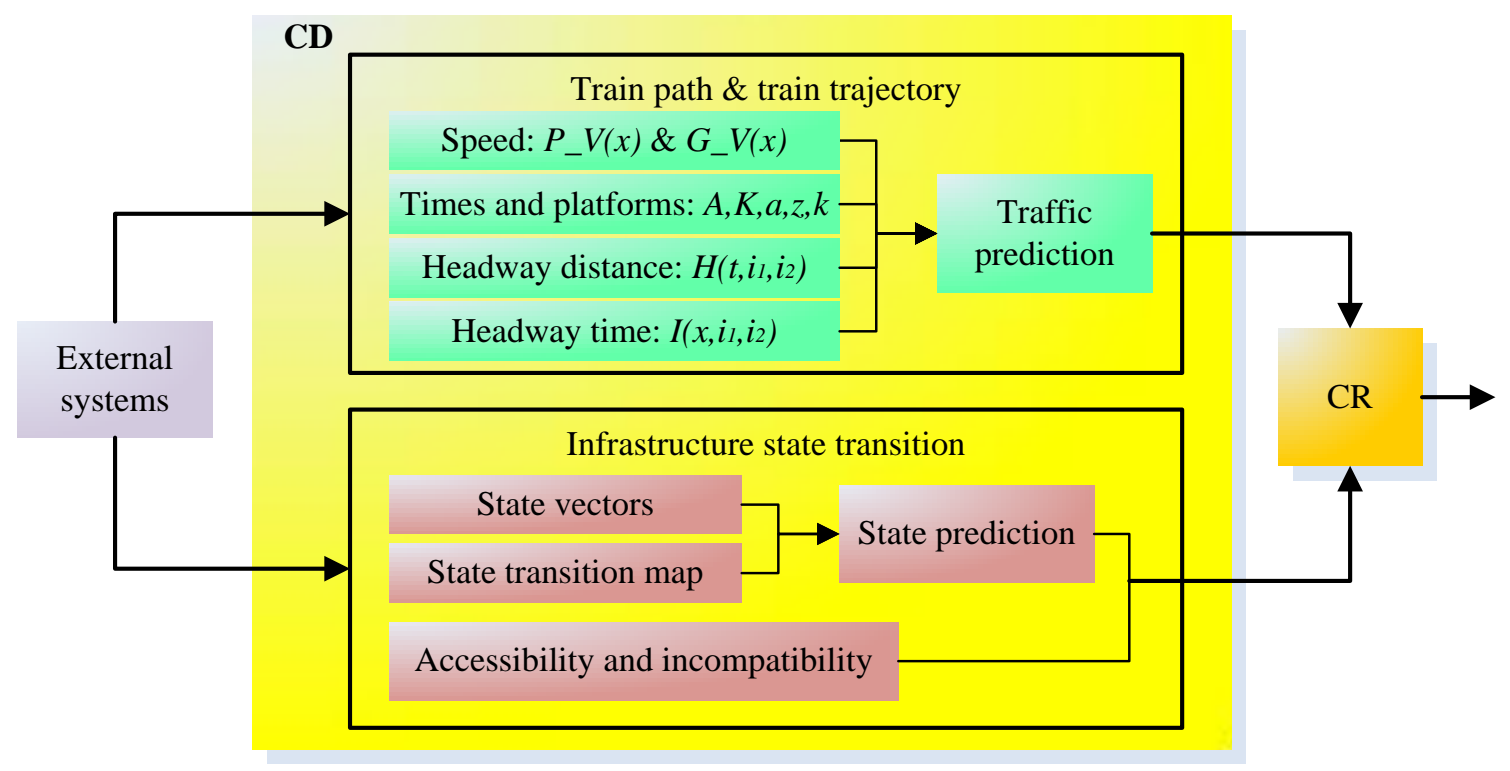

Figure 4-1 Methodological structure of conflict detection

Both approaches include a short time traffic prediction function. We assume $\omega$ is the prediction time horizon, which is taken into account by both approaches.

\subsection{Train trajectory approach}

\subsubsection{Train path domain and train trajectory}

According to the traffic model, the train position (usually in mileage) and the occupied track, train speed and the operation times are all tracked. They compose the core information of train trajectory.

In different ERTMS/ETCS application level, the train positioning function on-board may estimate the real-time position via different equipment and techniques. It is the precondition to apply the train trajectory approach to conflict detection that the train position information is available in the concerned railway system.

Considering $g^{i}(x, y, t, v)$ is a point on the actual trajectory of train. As defined in 3.3.1, $x$ is the mileage of the line and $y$ is a reflection of the relative (paralleled) position of track, $t$ is time and $v$ is the instantaneous speed. The two spatial dimensions 
are always restricted in joint definition, $(x, y) \in N(X, Y), N(X, Y)$ is the valid field of the actual railway (tracks) topology. More relations are also implied, such as distancetime diagram, time-speed profile and distance-speed profile, etc.

To the comparison end, train path $P^{i}$ can be correspondingly extended as $P^{i}(x, y, t, v)$ that satisfies the geographical restraint of railway network, the corresponding time requirements: arrival time set $A^{i}$, departure time set $D^{i}$ and track ID at all stations $K^{i}$, and the static derivative $P^{i}{ }_{-} V(x)$.

If we consider the case of train path in a section between two stations, where the occupied track $(y)$ will remain constant, a flexible domain of train path $P^{i}(x, y, t, v)$ is possible to be further extended with additional relaxation conditions. For each train the possible path $P^{i}(x, y, t, v)$ is not only a single profile but a series of derivate profiles depending on varying speed profiles and other train control parameters (acceleration and braking characteristics). Each derivate curve satisfies (4-1).

$$
P^{i}{ }_{-} x(t)=\int P^{i}{ }_{-} V(t) d t
$$

The relaxation also includes a reasonable time margin for delayed setting off from the origin position.

The most important factor in loosening the train path domain for a section is the variability of train speed profiles. The speed is dependent on the technical performance of the train, most restrictive value of speed limit, etc. An example is shown in Figure 4-2 where ample time margin and three regions in distance-time view are introduced. For a particular scheduled train,

- $P 1$ is the train-path estimated by highest efficient combination of parameters, which allows a train arrive at the next destination earlier than scheduled time when it sets off without delay.

- $\quad P 2$ is the train-path estimated by exact perfect combination of parameters which let the train arrive on time when it sets off without delay.

- $\quad P 3$ is the train-path estimated by conditional combination of parameters which let the train arrive on time when it sets off with a margin of delay.

In ideal condition, the delay margin of $P 3$ is the departure ample time margin for a train path, and the arrival time difference between $P 1$ and $P 2$ is the arrival ample time margin. In most of practical situations, the ample time margin changes dynamically, not always keeping the same value as that of departure or arrival. Then the possibly flexible domain of train path is represented as 'B12' (between $P 1$ and $P 2$, where a train is on time when it enters the section) and 'B23' (between $P 2$ and $P 3$ where a train is delayed when it enters the section). Figure 4-2 still shows a technique-hard region ' $A$ ' (above of $P 1)$ that is beyond the maximum speed and the acceleration limitation, and a delaytrended region ' $\mathrm{C}$ ' (below P3) where the train tends to fail to arrive on time at the next destination of the path. 


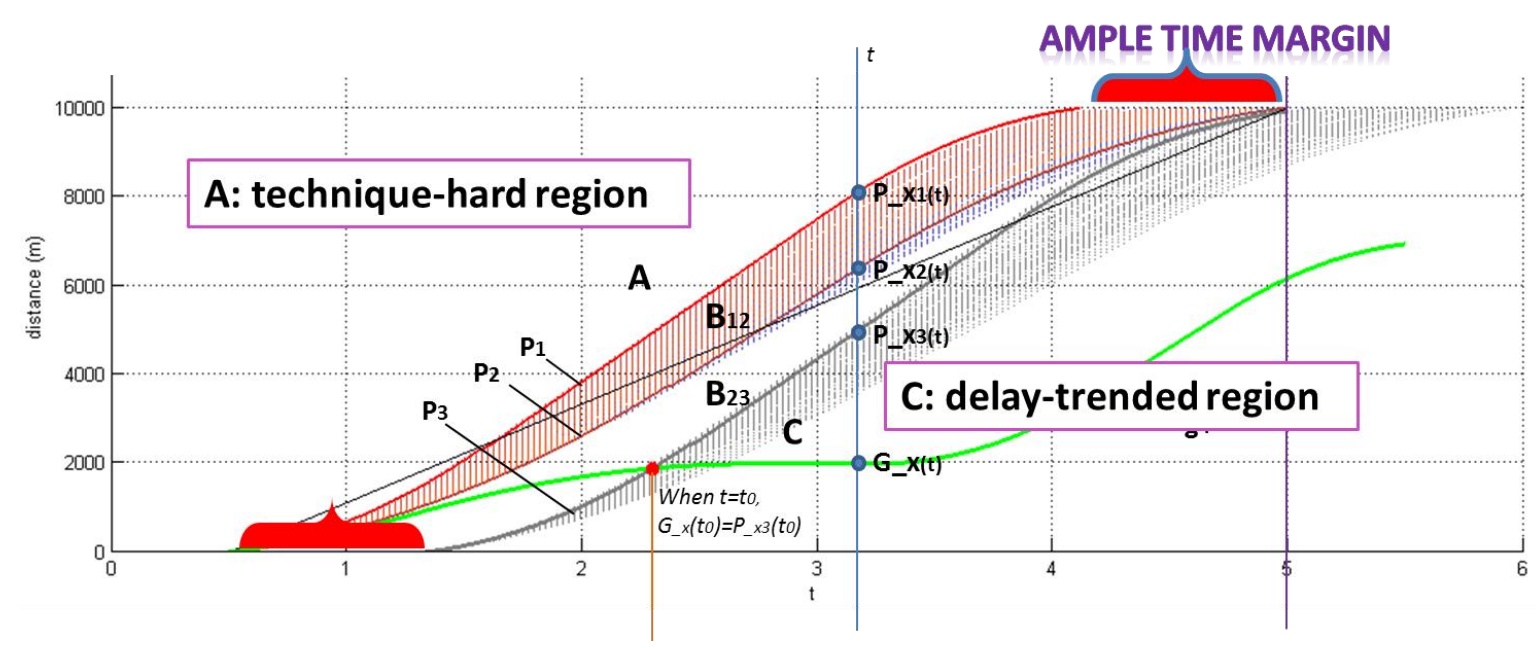

Figure 4-2 An illustration of technique-hard region (A), flexible domain (B12 and B23) and delay-trended region $(\mathrm{C})$

Generally, the actual trajectory $g^{i}(x, y, t, v)$ of a train is difficult to extend to region 'A' except of system update or technical breakthrough, which are long-term aspects and not under consideration in the approach. Normally, the actual trajectory is controlled to remain in the flexible domain $(\mathrm{B} 12+\mathrm{B} 23)$ so that it can probably realise the scheduled time-space target. However, not all $g^{i}(x, y, t, v)$ in the flexible domain can guarantee the on-time performance, e.g. the red point exampled in Figure 4-2.

\subsubsection{Deviations of train trajectory}

As the three-dimensional graphic representation of timetable (in 3.4.3), the train trajectory can also be represented in the same coordinate system. It allows the differences between actual train trajectory and planned train path in $(x, y, t)$ dimensions to be visualised. These differences contain time deviation, distance gap and unexpected track occupation, shown in Figure 4-3. Due to the dependent relationship between space and time, in most of cases a distance gap remains the same character as time deviation (e.g. qualitatively, when a train has a wide distance gap from its scheduled distance-time line, as well it gets an obvious delay in time dimension), except at a certain number of key points (e.g. entrances, stop signals, etc.) where sometimes a train cannot exceed. As mentioned above, the situation of unplanned track occupation can be first time detected and taken into consideration. 


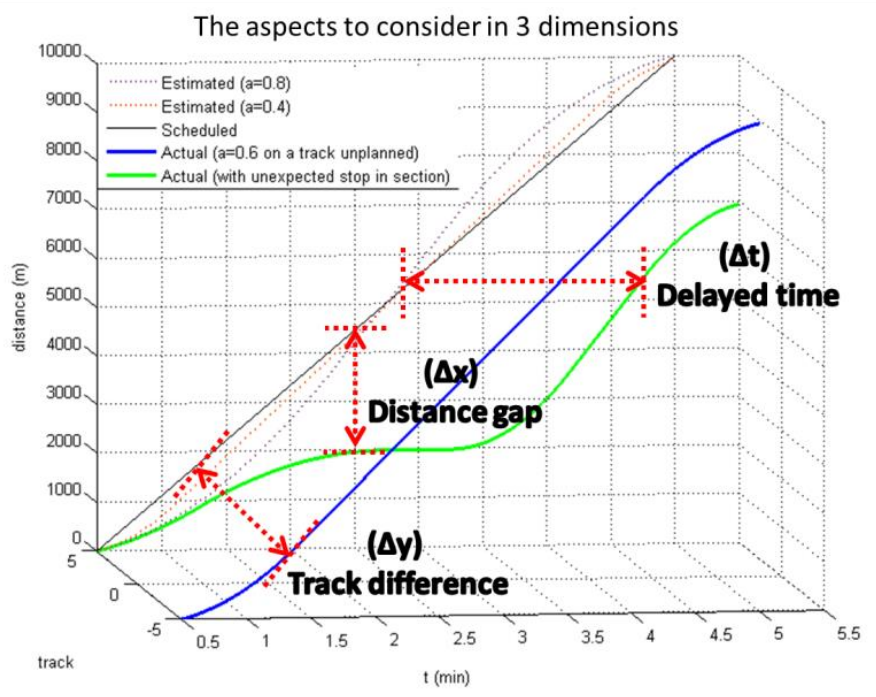

Figure 4-3 The comparison of scheduled and actual time-space profiles in xyt dimensions

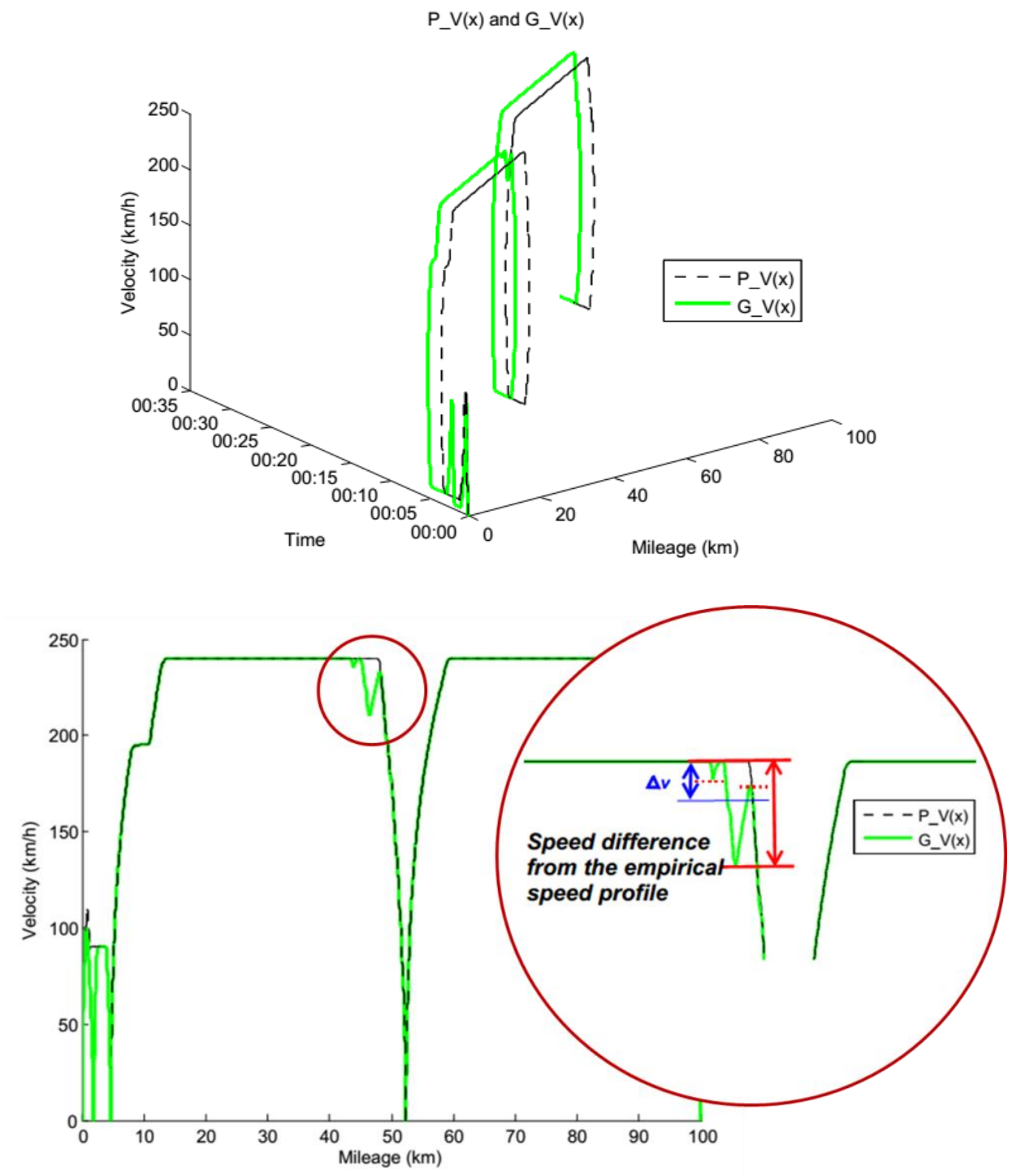

Figure 4-4 Train speed deviation from the reference speed profile in xtv and xv dimensions 
Furthermore, the velocity data of each train referring to its speed supervision system can also be displayed with the combination of distance and time, i.e. in $(x, t, v)$ dimensions. Figure 4-4 shows an example of (a single) train speed deviation from its empirical speed profile. Similar to the time deviation in distance-time view, the time deviation in time-speed view implies an early or a delayed train trajectory. By contrast, the speed deviation in distance-speed $(x v)$ view is not manifest of train delay or space gap. If the early or delayed value remains the same all the way, it is hardly observable the speed deviation in distance-speed $(x v)$ view. More exactly, the speed profile in distance-speed $(x v)$ view reflects the driving modes of the train in a given environment. Then the speed deviation in this view implies the adjustment of driving modes that in reverse causes immediate variations in other dimensions of the train trajectory.

Besides the speed deviation of each single train, the speed difference between each pair of successive trains is observable. For those successive trains whose empirical speed profiles in the coordinate system are parallel, as illustrated in Figure 4-5, the speed difference variation implies the subtle change in the headway relationship (headway time and headway distance). Note that being parallel is not a necessary condition but a common instance of multi-train plan. However, when the empirical speed profiles are initially different, the relative difference should also approximate a static function of position.

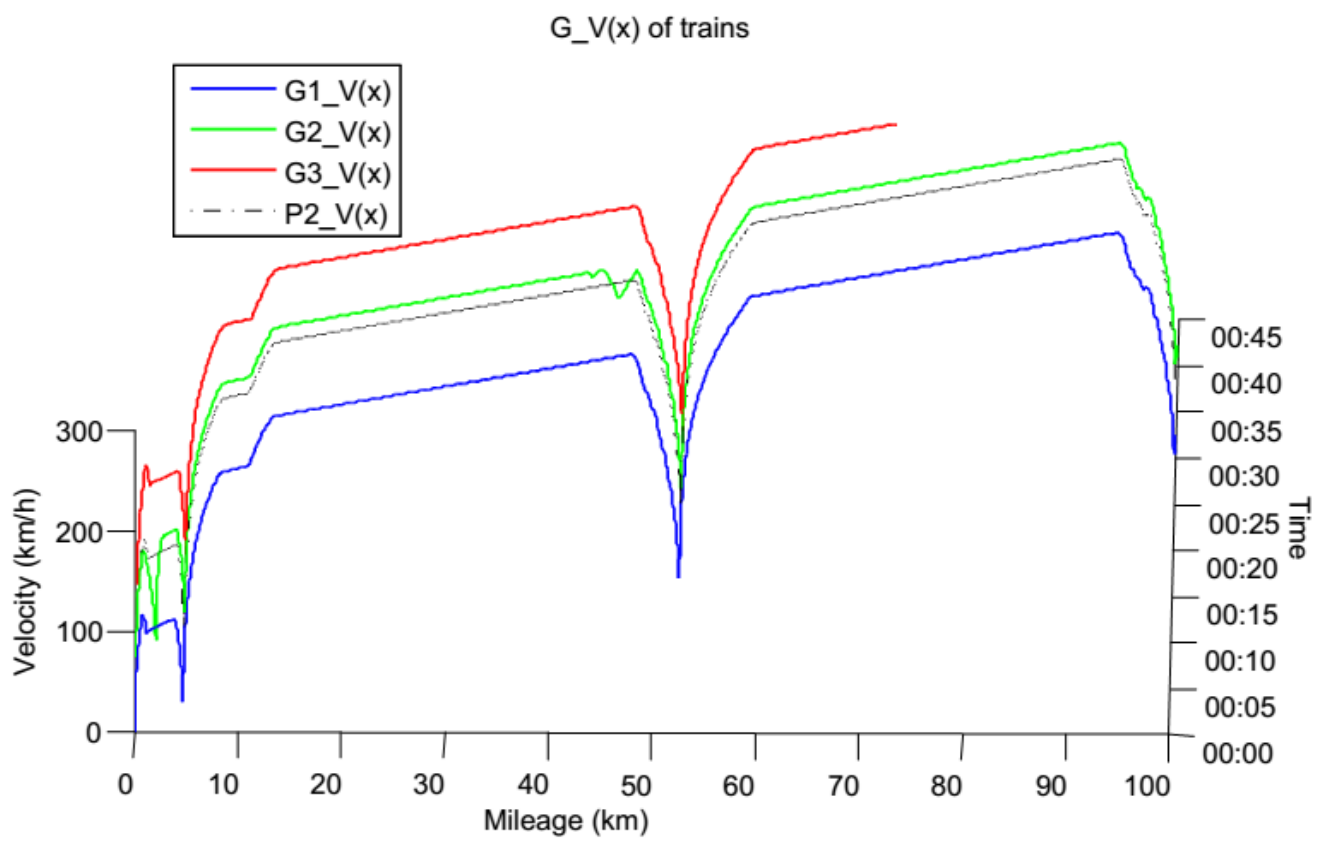

Figure 4-5 Multi-train trajectories in $x t v$ dimensions

Three categories of actions may be taken after the deviations of train trajectory (trajectories) are detected.

First, immediate warning of potential conflict if any of the following occurs:

$$
|\Delta y|>0
$$




$$
\left|g^{i_{1} \_} x t(t)-g^{i_{2}} \_x h(t)\right|<h\left(t, v_{1}, v_{2}\right)
$$

Here $\Delta y$ is the track difference between $g^{i}(x, y, ;)$ and $P^{i}(x, y, ;)$ referring to the same value of mileage. $h\left(t, v_{1}, v_{2}\right)$ is the minimum headway distance function of the succeeding train $i_{2}$ and the foregoing train $i_{1}$. It may have distinct definition according to different application levels of ERTMS/ETCS (under different signalling systems).

The second category is sending early warning meanwhile calling further traffic prediction that is introduced in 4.1.3. Actions to most conflict signs fall into this category.

- When the speed deviation coincides with the inequality (3-5), i.e. the deviation is larger than the given threshold $\Delta v$, the early warning activate the trajectory prediction procedure.

- When the absolute distance from the rear end of a foregoing train to the front end of the succeeding train is deviating from the defined headway $H\left(t, i_{1}, i_{2}\right)$, but not overstepping the minimum value of the headway restriction $h\left(t, v_{1}, v_{2}\right)$, further observation is also the response. In this case, a margin exist between $h\left(t, v_{1}, v_{2}\right)$ and $H\left(t, i_{1}, i_{2}\right)$.

- Other deviations corresponding to the inequalities (3-7, 3-8, 3-10).

The third category is ignoring the tiny deviations when they are not in the above cases.

\subsubsection{Train trajectory prediction}

In the prediction procedure, all involved trains are simulated separately so that possible conflicts are evaded. A cellular automaton method is applied to simulate the predictive speed and position of each individual train. In this method, the train movement is updated according to the following steps:

Step 1: Simulate one of the following driving mode.

- Acceleration: increase $g^{i} \_v(t)$, by the acceleration rate in service if $g^{i} \_v(t)<$ $\operatorname{MRSP}(x)$ and $g^{i}{ }_{-} v(t) \leq P^{i}{ }_{-} V(x)$, where $x=g^{i}{ }_{-} x h(t)$;

$g^{i}{ }_{-} v(t+1)=g^{i} \_v(t)+a^{i}$

where $a^{i}$ is the given value of acceleration or an acceleration function.

- Deceleration: decrease $g^{i} \_v(t)$ by the brake rate (service brake or emergency brake) in the distance to the end of movement authority if $g^{i} \_v(t)>$ $g^{i}{ }_{-} V l o a(t)$ or $g^{i} \_v(t)>P^{i}{ }_{-} V(x)$, where $x=g^{i}{ }_{-} x h(t)$;

$$
\begin{aligned}
& g^{i}{ }_{-} v(t+1)=g^{i}{ }_{-} v(t)-s b^{i}(v), \text { where } v=g^{i}{ }_{-} v(t) \\
& g^{i}{ }_{-} v(t+1)=g^{i}{ }_{-} v(t)-e b^{i}(v), \text { where } v=g^{i}{ }_{-} v(t)
\end{aligned}
$$


Where $s b^{i}(v)$ and $e b^{i}(v)$ are the speed dependent deceleration functions for service brake and emergency brake, respectively.

- Cruising: keep the same speed $g^{i}{ }_{-} v(t)$ if $g^{i}{ }_{-} v(t)=g^{i}{ }_{-}$Vloa $(t)$ or $g^{i}{ }_{-} v(t)=$ $\operatorname{MRSP}(x)$, where $x=g^{i} \_x h(t)$;

$$
g_{-}^{i} v(t+1)=g_{-}^{i} v(t)
$$

Step 2: Randomise the speed by increasing or decreasing the value of $g^{i} \_v(t+1)$ with randomization probability, where $g^{i}{ }_{-} v(t+1)>1$ and $-1 \leq p \leq 1$

$$
g^{i}{ }_{-} v(t+1)=g^{i} \_v(t+1)+p * a^{i} / 16
$$

Step 3: Realise the movement by

$$
g^{i}{ }_{-} x h(t+1)=g^{i}{ }_{-} x h(t)+\left(g^{i} \_v(t+1)+g^{i}{ }_{-} v(t)\right) / 2
$$

Update $g^{i}{ }_{-} x h(t), g^{i}{ }_{-} x t(t)$ and the predictive part of the trajectory.

Once the prediction function is called, the prediction procedure is uninterrupted in the prediction time horizon $\omega$. Then the predictive trajectories are analysed as described in 4.1.2.

\subsection{State transition approach}

The restrictions taken into account in the trajectory approach are more about the train speeds and safety separations on the interstation tracks. In other words, the route conflicts and operational time effects in station area might be grouped into the third category by using the trajectory approach. Thus, the infrastructure state transition approach can be specifically designed to apply to the stations where the topology is more complex and more (dispatching) operations are contained, yet the approach is also suitable to the non-station sections.

This approach is based on the infrastructure model. Before dynamic data collection and conflict estimation, well preparing of restrictive conditions is the prerequisite of effective conflict detection.

\subsubsection{State vector and hash table}

In a station section, we can collect the instantaneous state values of $o_{j, s}(t)$ and $r_{j, r}(t)$, and update corresponding state vectors. Take Figure 4-6 as a small example, 6 segments and 4 routes are in consideration. 


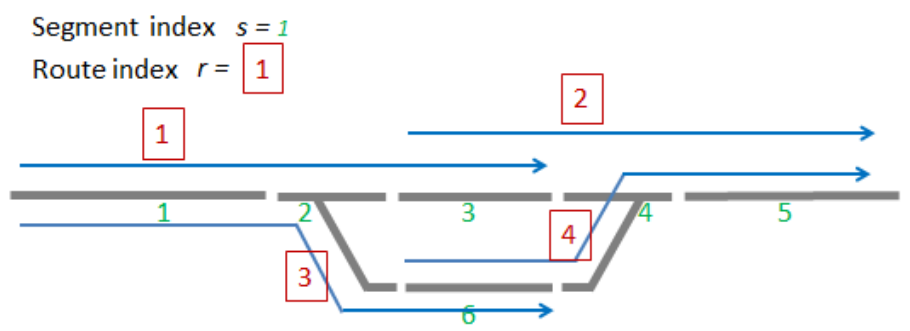

Figure 4-6 An example of 6 segments and 4 routes

As the binary characteristics of the state variables, a state vector is a binary sequence with a length equalling the total number of certain state type. In this case the segment state vector $s_{j_{-}}$vector $(t)$ is a sequence with the total length of 6 segment states. Since each state has 2 possible values, theoretically, there are $2^{6}$ possibilities of combination of the sequence even when the segment index order is determined. Although practically in a railway system only a certain percentage of them are logical and still less occur frequently in daily railway traffic, the length of each state sequence increases and the total state possibility number grows at an exponential rate with the increase of total segment number. To store all the states and fast access to the stored information of each existing state, a hash table is used to reflect the full feasible state vectors and frequent state vectors. We use the following hash function to calculate the key index of a segment state vector in its hash table.

$$
s_{\text {index }(t)}=\sum_{s} o_{j, s}(t) \cdot 2^{s-1}, \sum_{s} o_{j, s}(t) \neq 0
$$

Numerically, the hash index is the de decimal value of the binary representation of state vector. Table 4-1 shows how the hash function realises the bijection relationship between segment state vectors and hash indexes, except a zero vector. We consider the state vector of zeros is the entrance and the exit of a particular state vector set and not actually included in the hash table.

Table 4-1 Mapping relationship between segment state vectors and hash indexes

\begin{tabular}{ccccccc|c}
\hline \multicolumn{7}{c|}{ Segment index order (low-right) } & Hash index \\
\cline { 1 - 4 } Segment index & 6 & 5 & 4 & 3 & 2 & 1 & \multirow{2}{*}{$\begin{array}{c}o_{1,6} \cdot 2^{5}+o_{1,5} \cdot 2^{4}+o_{1,4} \cdot 2^{3} \\
+o_{1,3} \cdot 2^{2}+o_{1,2} \cdot 2^{1}+o_{1,1} \cdot 2^{0}\end{array}$} \\
\hline State & $o_{1,6}$ & $o_{1,5}$ & $o_{1,4}$ & $o_{1,3}$ & $o_{1,2}$ & $o_{1,1}$ & 24 \\
\hline State vector & 0 & 1 & 1 & 0 & 0 & 0 & \\
\hline
\end{tabular}

Similarly, the state vector $r_{j-}$ vector $(t)$ is a sequence as well with a length equal to the number of route states. A similar hash function shows below, where the route index order in the binary sequence is right-started.

$$
r_{\text {index }(t)}=\sum_{r} r_{q, r}(t) \cdot 2^{r-1}, \sum_{r} r_{q, r}(t) \neq 0
$$

A zero vector of route state is not included in the hash table either, but it always logically exists to indicate all the routes are clear. As defined in Figure 4-6 there are 4 
routes based on above 6 segments, $r_{\text {index }(t)}$ in this example depends on a 4-bit binary sequence. In accordance with the segment state transition for one train, Figure 4-9 shows the route transition map and corresponding hash table.

If we assume all segments in this example are longer than train length, then the complete set of segment state vectors for one train (the number of trains also affects the state vector, which will be discussed in next section) and corresponding unit state hash tables can be illustrated in Figure 4-7 (a) and (b). A state unit here is a possible state for a train to use the infrastructure at a moment.

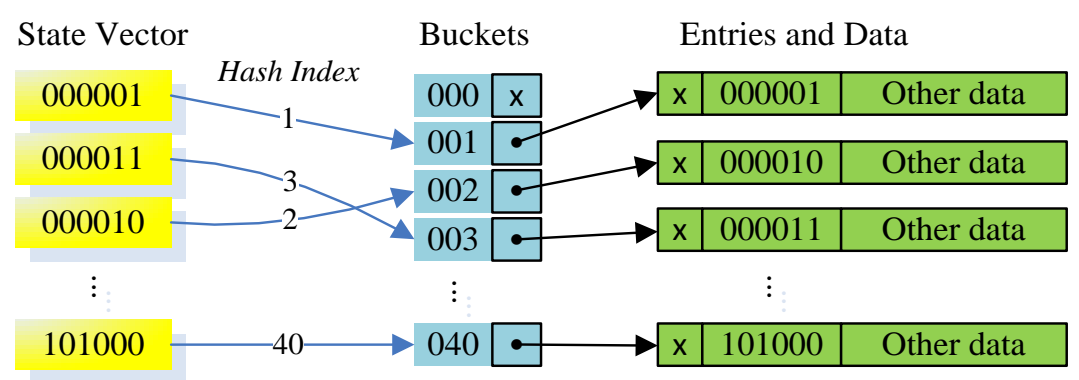

(a) Segment hash table

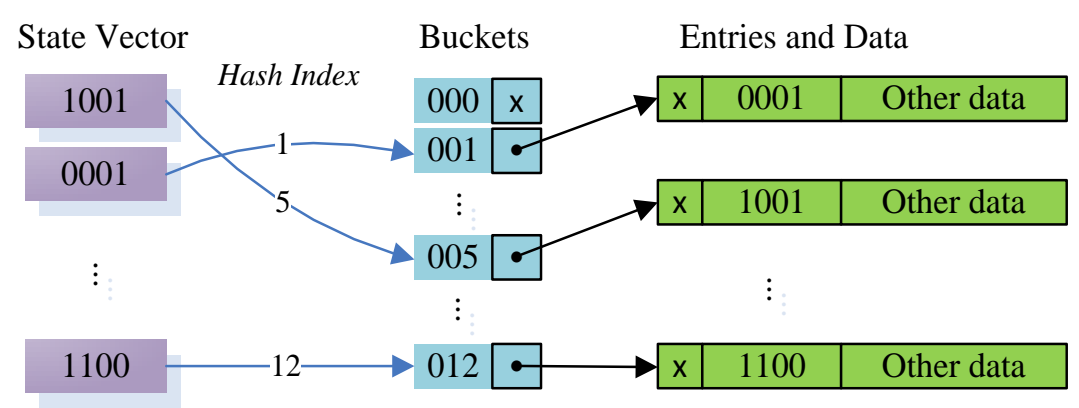

(b) Route hash table

Figure 4-7 Hash tables of segment vectors and route vectors (for one train)

Other data in Figure 4-7 means the information related with the state vectors, including average time to maintain to switch to another state vector, whether to check and record the arrival time and departure time, minimum waiting time, etc. For example, Table 4-2 shows the additional information supplemented to the segment vectors, where hash indexes are used to identify corresponding state vectors. E.g., if the current state is 000100 (with hash index 4), we can get that there is a stop of 10 or more than 10 seconds at this state, and with about 12 seconds more to land on this state and to switch from this state to the next one, state 001100 (with hash index 12). In this state actual arrival time $a_{q}^{i}$, departure time $d_{q}^{i}$ can be recorded to compare with the planned times. 
Table 4-2 State transition table with extra statistical properties (to Figure 4-6)

\begin{tabular}{l|llll|ll}
\hline $\begin{array}{l}\text { Current } \\
\text { state }\end{array}$ & $\begin{array}{l}\text { Check } \\
a / d\end{array}$ & $\begin{array}{l}\text { Wait } \\
\text { time }\end{array}$ & $\begin{array}{l}\text { Next } \\
\text { state_1 }\end{array}$ & $\begin{array}{l}\text { Time to next } \\
\text { state_1 }\end{array}$ & $\begin{array}{l}\text { Next } \\
\text { state_2 }\end{array}$ & $\begin{array}{l}\text { Time to next } \\
\text { state_2 }\end{array}$ \\
\hline 0 & $\mathbf{0}$ & $\mathbf{0}$ & 1 & 4.5 & 0 & 0 \\
1 & $\mathbf{0}$ & $\mathbf{0}$ & 3 & 8 & 0 & 0 \\
2 & $\mathbf{0}$ & $\mathbf{0}$ & 6 & 3.5 & 34 & 6 \\
3 & $\mathbf{0}$ & $\mathbf{0}$ & 2 & 5.5 & 0 & 0 \\
4 & 1 & 10 & 12 & 20 & 0 & 0 \\
6 & $\mathbf{0}$ & $\mathbf{0}$ & 4 & 5.5 & 0 & 0 \\
8 & $\mathbf{0}$ & $\mathbf{0}$ & 24 & 6 & 0 & 0 \\
12 & $\mathbf{0}$ & $\mathbf{0}$ & 8 & 5 & 0 & 0 \\
16 & $\mathbf{0}$ & $\mathbf{0}$ & 0 & 7.5 & 0 & 0 \\
24 & $\mathbf{0}$ & $\mathbf{0}$ & 16 & 4.5 & 0 & 0 \\
32 & 1 & 15 & 40 & 26 & 0 & 0 \\
34 & $\mathbf{0}$ & $\mathbf{0}$ & 32 & 4 & 0 & 0 \\
40 & $\mathbf{0}$ & $\mathbf{0}$ & 8 & 5 & 0 & 0 \\
\hline
\end{tabular}

To include the non-station section $\left(S_{j-}\right.$ type $\left.=0\right)$ into the scope to apply the approach, we can assume a track at the non-station section as several adjoining routes (disregarding of the exact number of segments). An example of unidirectional doubletracked section is shown in Figure 4-8.

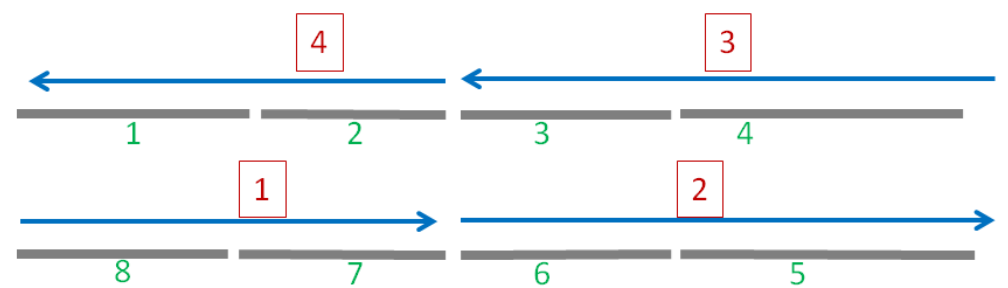

Figure 4-8 Illustration of segments and routes at a non-station section

\subsubsection{State transition map}

We have mentioned above that the transition relations are also implied in the hash table (in Figure 4-7 and Figure 4-9). The indexes of states are the key factors in a state transition relation, but they are not the only type of factors.

Figure 4-7 shows that the hash table can make the query of a state vector more efficient. In addition, hash indexes can also be contained in the cited information of related segment vectors, such as the columns of 'Next state_1' and 'Next state_2' shown in Table 4-2. This way of cross-reference among state vectors somehow reflects the transition relationships of segment states and route states. 
The cross-relationships of segment states embedded in Table 4-2 can be drawn in a graphic way, in Figure 4-9 (a). Edges connecting the states, so called transition relations, are not only directing from current states to the next ones, but also indicating the transition times (the $5^{\text {th }}$ and $7^{\text {th }}$ columns in Table 4-2). Similarly, Figure 4-9 (b) illustrates the route transition map according to the route hash table.

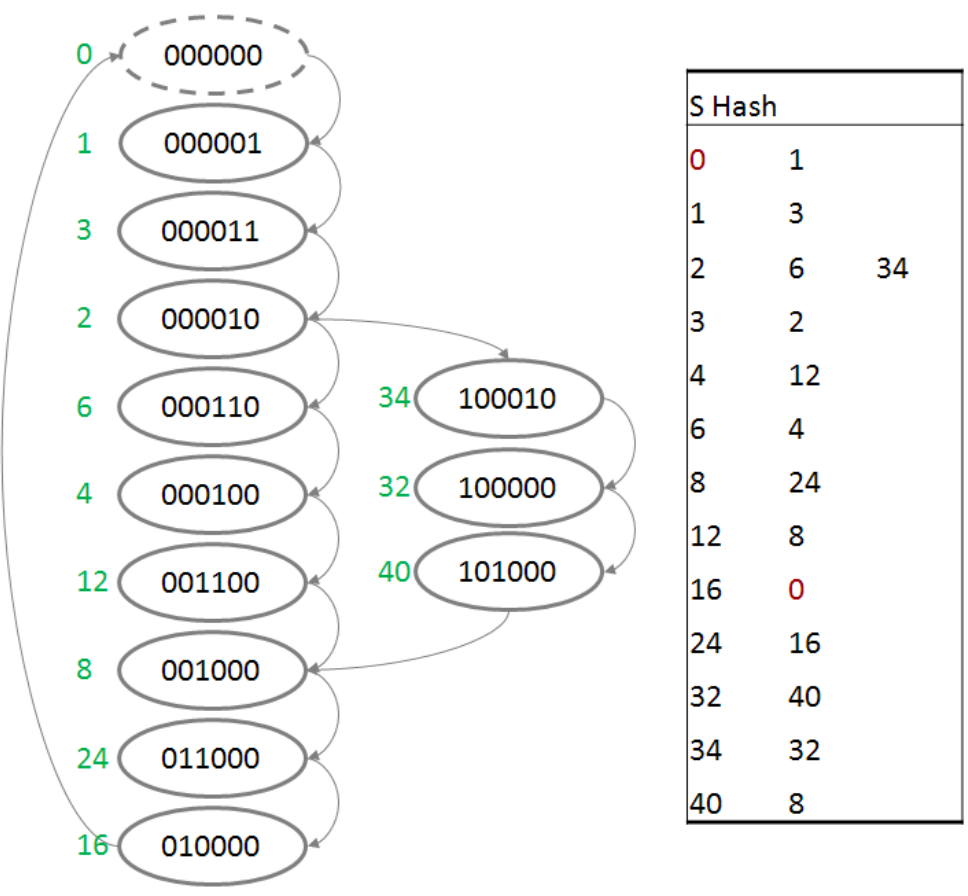

(a) Transition map of segment states

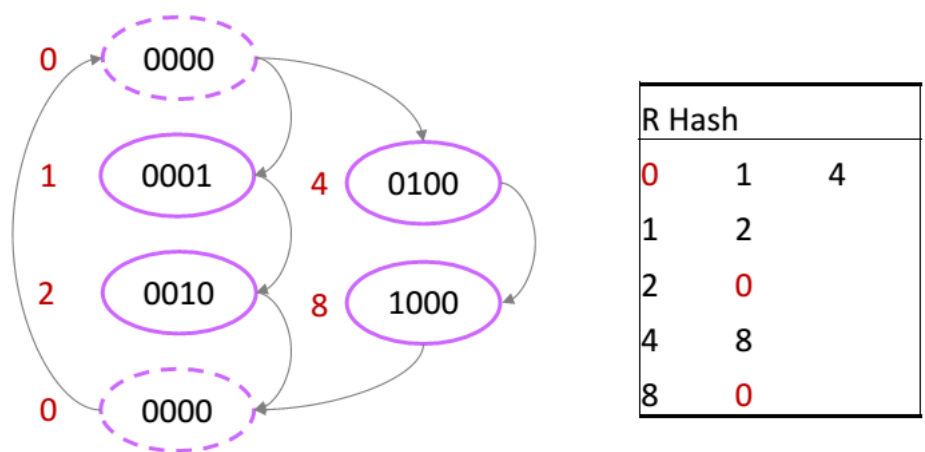

(b) Transition map of route states

Figure 4-9 State transition maps to the example in Figure 4-6 (for one train)

A route state transition map can be obtained directly from the collected data. Meanwhile it still can be drawn from the segment state transition map if the route state $r_{j, r}(t)$ is not directly available in some systems. Statistical time of one-step route state transition is the non-overlapping accumulation of the corresponding transition times of segment states that match to the same route state vector. Usually the time to next state of route is not as sensitive as that of segment. 
To represent the state of local traffic, composite state vector is introduced. The instant composite state can be either a partial state vector when there is only a single train in the concerned railway network (as illustrated above) or a linear combination of the partial state vectors of corresponding trains when there are several trains in the concerned area. Correspondingly, the state transition map based on the composite state vectors can vary according to the number of trains involved. Figure 4-10 illustrates three types of transition maps where the state vectors are quantised with the hash indexes:

(1) the one-train transition maps (to partial state vectors);

(2) the multi-train transition maps (to composite state vectors) which are obtained from historical data of normal traffic flow;

(3) and the composed multi-train transition maps which are generated from the timetable and one-train transition maps.

From Figure 4-10 we can find that types (2) and (3) are highly congruent. It means that the daily traffic state in a certain railway network has an obviously regular transition trend, mainly due to the well predefined schedule. Meanwhile, it also implies that both the empirical (historical data-based) state transition map and the timetable based state transition map can be employed to estimate the states in a short period of time.
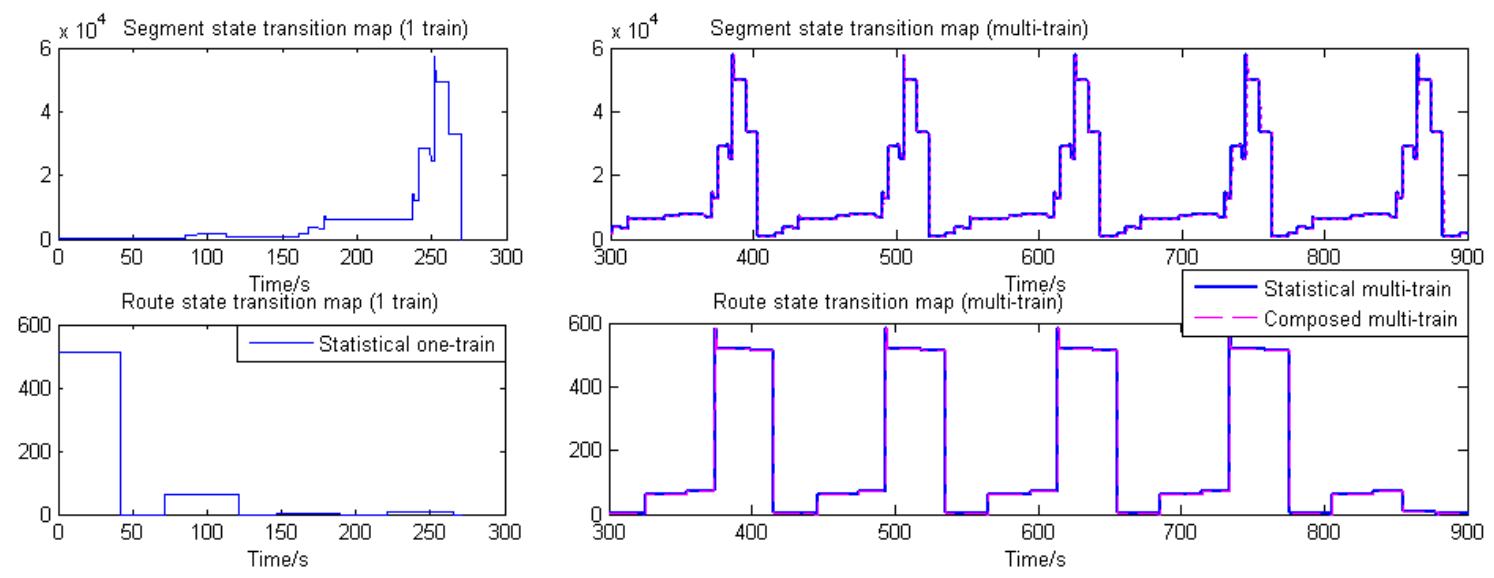

Figure 4-10 State transition maps represented by the hash index value of state

\subsubsection{Accessibility matrix and Incompatibility matrix}

The number of valid state vectors grows when the total number of segments or routes increases. And the transition map expands particularly while the number of involved trains increases as well, until the limitation of capacity of the railway network. Nevertheless, the relationship between one train and the infrastructure is the most essential. We use a partial state vector to indicate the instantaneous relationship between a particular train and its environment (usually a section) and a composite state vector to represent the situation where more than one train are simultaneously in the same section.

The one-step accessibility matrix and incompatibility matrix of route are exacted from the interlocking logic of a railway network. One-step accessibility matrix is a particular form to explicate the route compatibility (for the same train) at the next step 
and oriented to a partial route state vector, while the incompatibility matrix is more often to explicate the route compatibility (for different train routes) at the same time and the object is composite route state vector (or matrix).

A common form of one-step accessibility matrix $A 1, a_{r_{1} r_{2}} \in A 1$, can be defined as follows.

$$
A 1=\left[\begin{array}{ccc}
\varepsilon & \cdots & a_{1 m} \\
\vdots & \ddots & \vdots \\
a_{m 1} & \cdots & \varepsilon
\end{array}\right], 1 \leq r_{1}, r_{2} \leq m, r_{1} \neq r_{2}
$$

where $m$ is the number of routes, $a_{r_{1} r_{2}}$ is the accessibility of route $r_{2}$ when the current route $r_{1}$ is in process, e.g. $a_{r_{1} r_{2}}$ values 1 when it is possible for route $r_{2}$ to be operated after route $r_{1}$, and $a_{r_{1} r_{2}}$ is 0 if route $r_{2}$ is not accessible after route $r_{1}$. We still define a positive constant $\varepsilon$ to represent that a route remains in its current state, i.e. $a_{r_{1} r_{2}}=$ $\varepsilon\left(r_{1}=r_{2}\right)$.

In a similar form, matrix $I$ expresses the incompatibilities of routes for different trains at the same time.

$$
I=\left[\begin{array}{ccc}
\varepsilon & \cdots & i_{1 m} \\
\vdots & \ddots & \vdots \\
i_{m 1} & \cdots & \varepsilon
\end{array}\right], 1 \leq r_{1}, r_{2} \leq m, r_{1} \neq r_{2}
$$

In contrast, $i_{r_{1} r_{2}}$ equals 0 if it is compatible for route $r_{2}$ of a second train $\bar{l}$ to be set when route $r_{1}$ is occupied or reserved by train $i$, otherwise $i_{r_{1} r_{2}}$ equals 1 .

A one-step accessible route vector $a_{-} T_{i}(t+1)$ and a current incompatible route vector $i c_{-} T_{\bar{l} i}(t)$ can be obtained by (4-14) and (4-15), respectively.

$$
\begin{aligned}
& a_{-} T_{i}(t+1)^{\prime}=A 1^{\prime} \cdot r_{j-} \operatorname{vector}(i, t)^{\prime} \\
& i c_{-} T_{\bar{I} i}(t)^{\prime}=I^{\prime} \cdot r_{j_{-}} \operatorname{vector}(t)^{\prime}
\end{aligned}
$$

Here $r_{j_{-}}$vector $(i, t)$, the partial state vector of train $i$, is distinguished from the composite state vector $r_{j_{-}}$vector $(t)$. Note that in this approach the symbol $(t+1)$ is the next time stage of the current, not exactly the time with the absolute sum value of $t$ and 1 .

If in Figure 4-6 the route map has the accessibility and incompatibility matrices as follows.

$$
A 1=\left[\begin{array}{cccc}
\varepsilon & 0 & 0 & 0 \\
1 & \varepsilon & 0 & 0 \\
0 & 0 & \varepsilon & 0 \\
0 & 0 & 1 & \varepsilon
\end{array}\right], I=\left[\begin{array}{cccc}
\varepsilon & 0 & 1 & 0 \\
0 & \varepsilon & 0 & 1 \\
1 & 0 & \varepsilon & 0 \\
0 & 1 & 0 & \varepsilon
\end{array}\right]
$$


If the current route state vector of train $i$ is 0001 , we can get the two vectors in (4-14a) and (4-15a).

$$
\begin{aligned}
& a_{-} T_{i}(t+1)^{\prime}=\left[\begin{array}{llll}
\varepsilon & 1 & 0 & 0 \\
0 & \varepsilon & 0 & 0 \\
0 & 0 & \varepsilon & 1 \\
0 & 0 & 0 & \varepsilon
\end{array}\right] \cdot\left[\begin{array}{l}
0 \\
0 \\
0 \\
1
\end{array}\right]=\left[\begin{array}{llll}
0 & 0 & 1 & \varepsilon
\end{array}\right]^{\prime} \\
& i c_{-} T_{\overline{l i}}(t)^{\prime}=\left[\begin{array}{llll}
\varepsilon & 0 & 1 & 0 \\
0 & \varepsilon & 0 & 1 \\
1 & 0 & \varepsilon & 0 \\
0 & 1 & 0 & \varepsilon
\end{array}\right] \cdot\left[\begin{array}{l}
0 \\
0 \\
0 \\
1
\end{array}\right]=\left[\begin{array}{llll}
0 & 1 & 0 & \varepsilon
\end{array}\right]^{\prime}
\end{aligned}
$$

Generally, the route states corresponding to the elements other than 0 or ' $\varepsilon$ ' in $a_{-} T_{i}(t+1)$ and $i c_{-} T_{\overline{l i}}(t)$ have to be checked. For example the result in (4-14a) indicates that if route 1 is reserved or occupied by train $i$ then only route 2 can be the next route of train $i$. So route 2 has to be prepared for train $i$ at next stage. Otherwise, a potential conflict between train $i$ and route 2 is to be identified. At the same time, the result in (4-15a) indicates that route 3 is not available for other trains at this moment (before route 1 is released). If any other train $\bar{l}$ is entering route 3 , the pair of train $i$ and $\bar{l}$ are conflicting.

A route state transition map of each train agrees with the one-step accessibility of route. And a composite state transition map is subject to the compatibility restrictions.

\subsubsection{Conflict matrix}

A conflict matrix $C(t)$ of each time step is defined,

$$
C(t)=\left[\begin{array}{ccc}
c_{1 m} & \cdots & c_{11} \\
\vdots & c_{i r} & \vdots \\
c_{\rho m} & \cdots & c_{\rho 1}
\end{array}\right], 1 \leq i \leq \rho, 1 \leq r \leq m
$$

where $\rho$ is the total number of trains, and $c_{i r} \in C(t)$ equals 1 if the state of a route $r$ is not satisfied the restriction of accessible $a_{-} T_{i}(t+1)$. If $1 \leq \bar{\imath} \leq \rho$ and the state vector $r_{j-}$ vector $(t)$ is not satisfied the compatibility requirement $i c_{-} T_{\bar{l} i}(t), c_{\bar{l} r} \in C(t)$ equals 2 . Otherwise $c_{i r}$ and $c_{\bar{\imath} r}$ equals 0 . The detailed conflict information $c^{i}(j, r, t, v, \vec{\imath})$ can be obtained if $c_{i r} \neq 0$. And $\bar{l}$ is the train conflicting with $i$ if exists $c_{\bar{\imath} r}=2$ in column $r$ of $C(t)$, or $\bar{l}$ can be empty if there is not any $\bar{l} \neq i$ in column $r$ of $C(t)$ satisfying $c_{\bar{l} r}=2$.

\subsubsection{State prediction}

Railway traffic usually is well planned. Despite of any disturbances or delays, it has to be controlled to recover as minimum deviation from the plan as possible. So the daily traffic flow has a generally regular trend. As mentioned in 4.2.2, the ideal state transition maps employed to represent the normal traffic state are regular as well. It is the basis to use the transition maps as references in state prediction and abnormal state detection. 
Figure 4-11 shows how state presentations, state transition maps and relation matrices are synthesised to predict the traffic state.

First, the following static parameters and constraints are prepared in advance:

- Configurations about the defined topology of infrastructure, initial definitions for segments and routes and the hash functions used in transition maps.

- The relation matrices $A 1$ and $I$ based on the configurations and definitions, which mainly represent the route restrictions.

- Hash tables and state transition maps that are generated offline.

- The time step of data collection and the prediction horizon $\omega$.

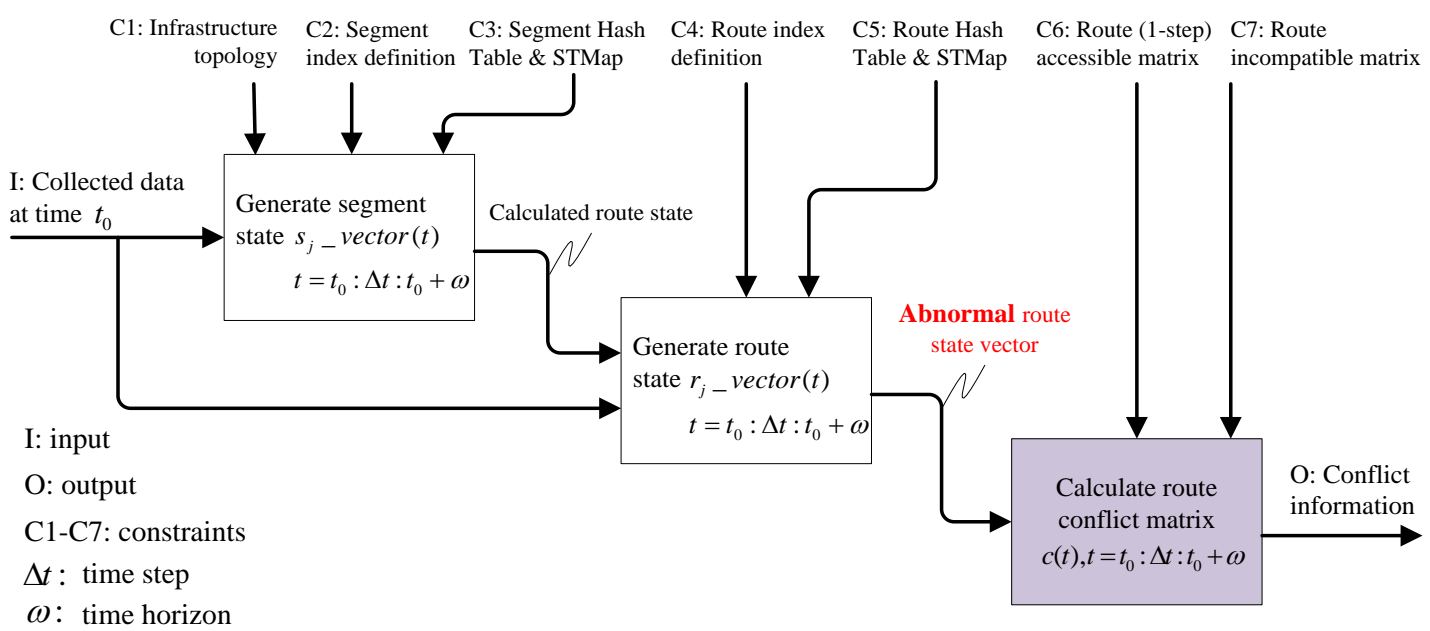

Figure 4-11 State prediction with determined structural constraints

Then instantaneous states of segment and route are extracted dynamically from the collected data (as mentioned in 4.2.2, route state vector can also be calculated by updated segment vector). Based on them, the state prediction procedure takes the following steps:

Step 1: Generate the current state vectors $s_{j_{-}}$vector $\left(t_{0}\right)$ and $r_{j}$ vector $\left(t_{0}\right)$ and calculate hash indexes according to corresponding hash functions, at the collection time $t_{0}$.

Step 2: Compare with the hash index values in the transition maps, referring to the additional information in corresponding hash tables if necessary.

- If the current route state vector is abnormal, go to Step 3.

- If the current route state vector matches to that in the route state transition map, go to Step 7. 
Here two types of abnormality are firstly detected: new state which is not recorded in the route hash table and the state taking a very different transition time compared with the one in the route state transition map.

Step 3: Calculate the current incompatible route vector $i c_{-} T_{\bar{i} i}\left(t_{0}\right)$ by (4-15) and check the route compatibility.

- If the route $r$ for train $i$, corresponding to the element that is not either 0 or $\varepsilon$ in $i c_{-} T_{\bar{l} i}\left(t_{0}\right)$ is not available, $c_{i r} \in C\left(t_{0}\right)$ equals 2 . Immediately warning of the route conflict (IC) and go to Step 7.

- Else if all elements in $i c_{-} T_{\bar{l} i}\left(t_{0}\right)$ are 0 or $\varepsilon$, let the prediction time $t=t_{0}$ and go to Step 4.

Step 4: Estimate the next state vector $r_{j_{-}}$vector $(t+1)$ and remaining time to the next state $\Delta t_{-} 2_{-}$next, according to the state transition map.

- If $t+\Delta t_{-} 2_{-}$next $<t_{0}+\omega$, go to Step 5.

- Else if $t+\Delta t \_2 \_n e x t \geq t_{0}+\omega$, go to Step 7 .

Step 5: For each train, calculate one-step accessible route vector $a_{-} T_{i}(t+1)$ by (4-14) and check the route availability at time $t+\Delta t_{-} 2_{-}$next.

- If $r_{j-}$ vector $(t+1)$ does not satisfy the $a_{-} T_{i}(t+1), c_{i r} \in C(t+1)$ equals 1 . Warning of the predictive route conflict (A1) and go to Step 6.

- Else if $r_{j}$ vector $(t+1)$ satisfies the $a_{-} T_{i}(t+1)$, directly go to Step 6.

Step 6: Calculate the incompatible route vector $i c_{-} T_{\overline{l i}}(t+1)$ by $(4-15)$ and check the route compatibility at time $t+\Delta t_{-} 2_{-} n e x t$.

- If the route $r$ for train $i$, corresponding to the element that is not either 0 or $\varepsilon$ in $i c_{-} T_{\bar{l} i}(t+1)$ is not available, $c_{i r} \in C(t+1)$ equals 2 . Warning of the predictive route conflict (IC) and go to Step 7.

Else if all elements in $i c_{-} T_{\bar{l} i}(t+1)$ are 0 or $\varepsilon$, let the prediction time $t=t+$ $\Delta t \_2 \_n e x t$ and go to Step 4.

Step 7: Wait until the next cycle of data collection and update time $t_{0}$.

\subsection{Integration in detection}

Train trajectory approach and state transition approach can be used separately, as well be combined in traffic prediction and conflict detection. 


\subsubsection{Integrated detection}

In Step 4 of state prediction procedure, the next state vector $r_{j_{-}}$vector $(t+1)$ with the remaining time $\Delta t_{-} 2_{-} n e x t$ is estimated via the empirical state transition map. The key to integrate the two approaches is estimating the next state vector $r_{j_{-}}$vector $(t+1)$ based on the prediction of train movements in the same prediction horizon $\omega$.

The integrated way of conflict detection is shown in the pseudocode in Figure 4-12. Different from that in state transition approach, $\Delta t_{-} 2_{-}$next is not estimated in the integrated approach. Instead, the prediction time step of train movement is used. When at two successive time steps $t$ and $(t+1)$, the predicted train trajectories produce $r_{j-}$ vector $(t+1) \neq r_{j_{-}}$vector $(t)$, state stage changes. The state transition map is only used as the reference to detect abnormal states. Early warnings of deviation in predicted trajectories and potential conflict information obtained in further assessment (headway restriction, route accessibility and compatibility) will be reported in detail.

\subsubsection{Potential conflicts}

The integrated approach in Figure 4-12 copes with the prediction and detection results in two manners: early warnings of significant deviation and the identified conflicts. They are respectively coded in E\#\#\# and C\#\#\#. Particularly, the information about interrelated trains is coded in Ex\#\# or Cx\#\#. Table 4-3 enumerates the codes and messages probably reported in the detection results.

Table 4-3 Coding of the results reported in conflict detection procedure

\begin{tabular}{lll}
\hline Message code & Judgement rule & Remarks \\
\hline E001 & Significant time deviation $\Delta t$ & Refer to (3-7), (3-8). \\
E002 & Track difference $\Delta y$ & Refer to (4-2), (3-9) \\
E003 & Significant speed deviation & Refer to (3-5) \\
Ex04 & Headway distance less than planned & Refer to (3-6) \\
Ex05 & Headway time less than planned & Refer to (3-10) \\
E\#06 - E\#19 & & Reserved \\
Cx20 & Headway distance less than minimum & Refer to (4-3) \\
C021 & Route unavailability & $\begin{array}{l}\text { Refer to the elements in } \\
C(t) \text { that value 1 }\end{array}$ \\
Cx22 & Route incompatibility & $\begin{array}{l}\text { Refer to the elements in } \\
C(t) \text { that value 2 }\end{array}$ \\
C\#23 & & Reserved \\
\hline
\end{tabular}

* E\#06 - E\#19 and codes from C\#23 are reserved for any other reported results.

On average, the detection sensibility of each conflict sign or conflict is ordered (from most to least) as: E002, E003, E001, Ex05 or Ex04, Cx20, C021, Cx22. On the contrary, the order of urgency is almost in a converse way, except E002 that the track difference is most urgent to cope with among all the early warning messages.

All reported messages are available dynamically to the traffic controller or similar control system. Each message basically consists of the detection time $t_{0}$, the estimated 
conflicting time $t$ and location (route $r$, segment $s$ or absolute position $(x, y)$ ), and the trains involved in the conflict.

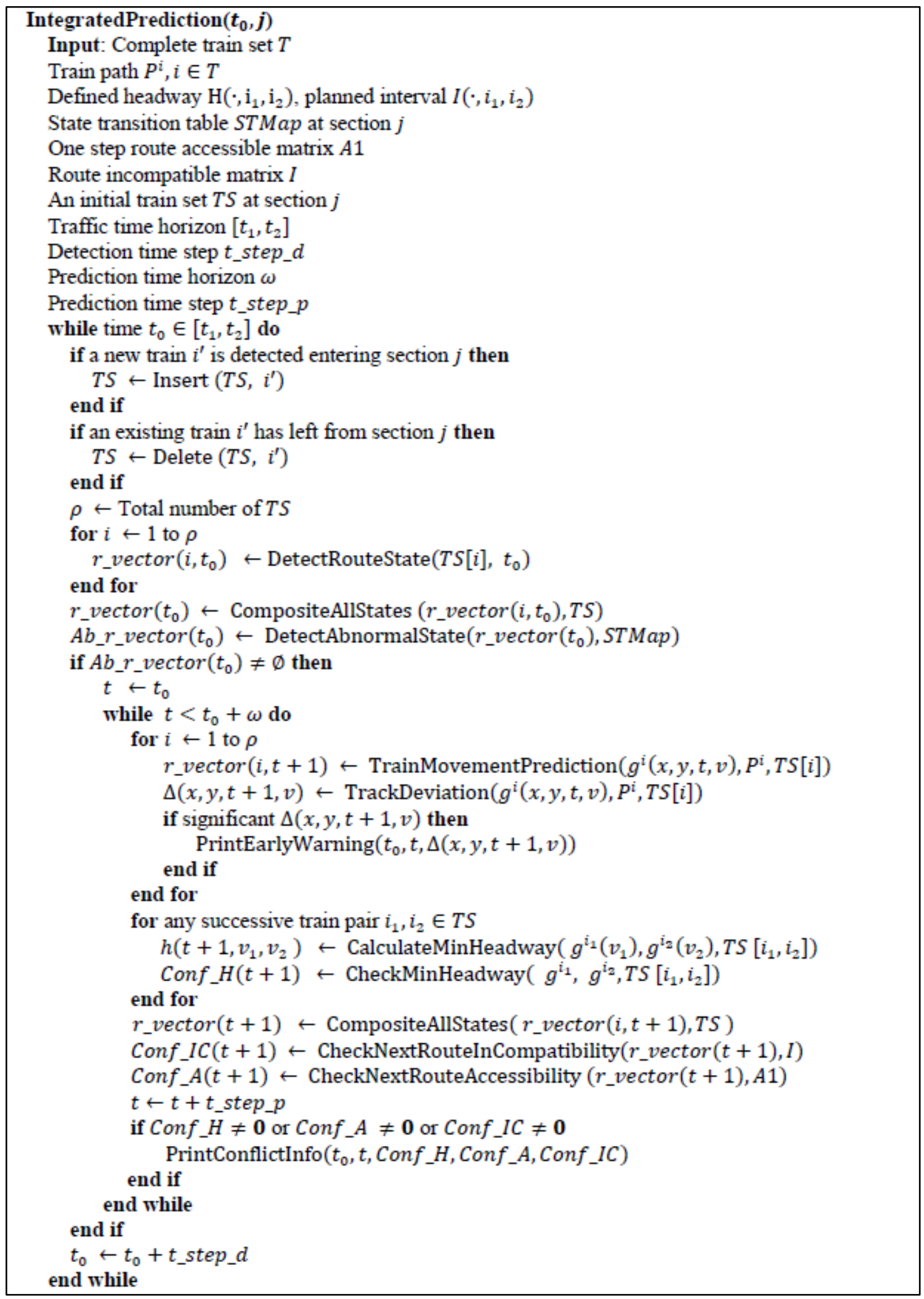

Figure 4-12 Pseudocode of the integrated approach for traffic prediction and conflict detection 


\subsection{Conclusions}

Train trajectory approach and state transition approach are presented to detect potential conflicts in this chapter. Both approaches are based on the knowledge of the target (timetable and its derivations) and current collected data.

The precondition to use the train trajectory approach is the availability of the train path and continuously detected train positioning information. Similarly, the state transition maps, restrictive matrices and the real-time track availability information are required. On these conditions, the specific approach selection may vary from one railway system to another. However, where the train trajectory approach is feasible, generally, the state transition approach is fit the system as well.

Conflicts are classified with different codes and detailed judgement rules. These rules are taken into account in the proposed approaches. Conflict signs or early warnings of deviation can be confirmed in the potential conflict information or excluded if they are prevented to develop into any route conflict. Extended, the headway conflict (coded in Cx20) essentially ends with route conflict. Thus, one strategy to prevent conflicts is to prevent the conversion from early warnings to route conflicts.

Other related topics that have not been discussed in detail in this chapter but might be interesting still include:

1) The structure definition of the infrastructure in state transition approach

Structure definition is allowed to be changeable. Reasonable definition favours the observation and even the division of state transition maps.

2) Hash tables and hash functions

Hashing method is used in the state transition approach for the following reasons:

First, hash function realises the converting between individual states and the whole state (vector). In traditional monitoring system, the instantaneous states or state logs are usually oriented to each individual (segment or route) and they seem to be disorderly. By converting all individual states (binary sequence) to a whole state (decimal value) in the same time dimension, we can observe that the states are periodically regular at a general level. Conversely, by converting a hash state value to a binary sequence, the structural restrictions (matrices) can be applied and the individual indexes can be located.

Second, in dynamic detection the transition maps and hash tables are accessed frequently, proper hash functions may save the time consumption. 
Finally, hash functions are decidable according to the system complexity and other conditions.

3) The relationship between the blocking time theory and the state transition (time) approach proposed in this chapter. 



\section{Chapter 5}

\section{Conflict resolution}

Local routing and variable scope rescheduling approaches are presented for conflict resolution. 


\section{Chapter 5 Conflict resolution}

Corresponding to the detected conflicts, Figure 5-1 presents a methodological structure of conflict resolution and prevention.

In this structure, two method layers are considered. First layer is to solve the routing problem in station area when potential route conflicts are detected. And the second layer is to prevent potential conflict propagation in a wider area via rescheduling technique.

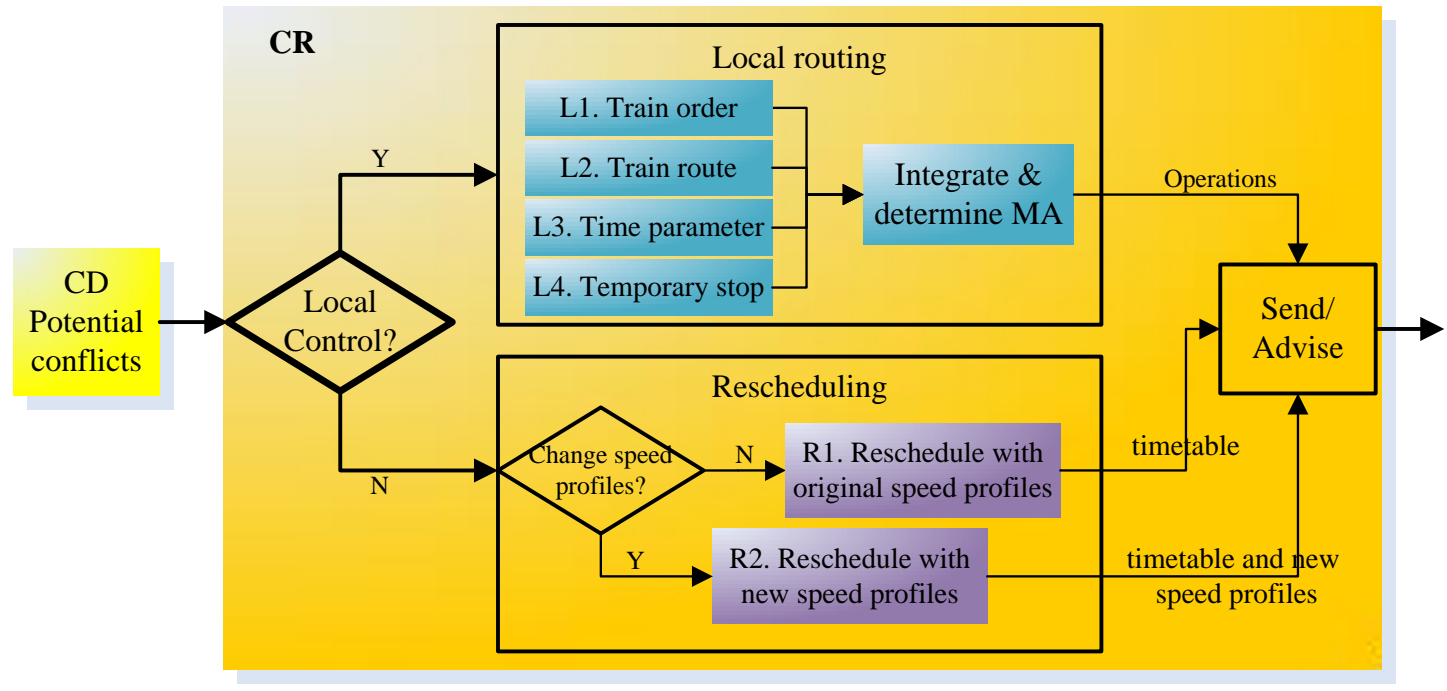

Figure 5-1 Methodological structure of conflict resolution

It is also worth making use the efficient algorithms and methods that have been applied to routing and rescheduling in conflict resolution.

\subsection{Local routing adjustment}

The local routing problem with different preconditions is firstly formulated as a series of mixed integer linear programs.

With regard to the times of train $i$ at station $q$, only the scheduled arrival and departure times $A_{q}^{i}$ and $D_{q}^{i}$, or passing times (here we set $A_{q}^{i}=D_{q}^{i}$ to indicate a passing case) are specified by the timetable. The more microscopic times to enter all segments are not determined. They are unpractical to be defined in a general timetable but considered in real time based on the actual traffic situation, in order to make reasonable decisions in local routing. Thus a type of auxiliary variable $t_{s}^{i}, s \in R_{r}^{i}$ is set to denote the exact time to enter a specific segment $s$ of train $i$. Correspondingly, a type of statistical parameter $B_{S_{1} s_{2}}^{i}$ is imposed to represent the minimum running time for the type of train $i$ from its head enters segment $s_{1}$ till it enters segment $s_{2}$. Figure 5-2 shows 
this group of definition, where we can see an extra $t_{o}^{i}$ that represents the entering time to the next section. When the segment $s$ is the last segment for train $i$ to exit from station $q$, the minimum running time is represented as $B_{S o}^{i}$. We assume the time of train $i$ entering the segment of platform $t_{s_{k}}^{i}$ adding a converted time of the train length $C^{i}$ as the estimated value of actual arrival time $a_{q}^{i}$ (e.g. with the segment $s_{3}$ being the platform $k$ of train $i$ in Figure 5-2, we accept $\left(t_{3}^{i}+C^{i}\right)$ as the estimated value of $\left.a_{q}^{i}\right)$, and the time entering the next segment of platform as the estimated value of departure time $d_{q}^{i}$ (e.g. $t_{4}^{i}$ in Figure 5-2).

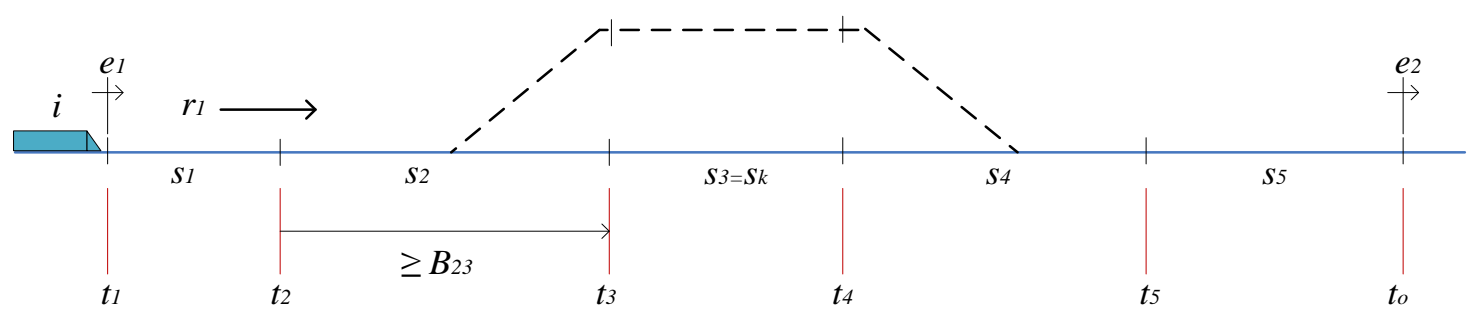

Figure 5-2 Train times at segment level

Other symbol definitions are in accordance with those in Chapter 3 and Chapter 4 .

In the scope of a local station $q$, the problem under different preconditions may follow one or more of the following objective functions.

$$
\begin{aligned}
& \min \sum_{i}^{T S_{q}} z_{q}^{i}, T S_{q} \subset T \\
& \min \sum_{i}^{T S_{q}} t_{o}^{i}-\tau_{o}^{i}, T S_{q} \subset T
\end{aligned}
$$

$T S_{q}$ is the involved train set at station $q$. The function (5-1) minimises the total arrival delay of trains at station $q$ and (5-2) minimises the total exit delay value when the trains exit from the station and enter to the next section of station $q$. The reference time $\tau_{o}^{i}$ marks the planned head-in time of train to the next section (or head-out time from the current station).

However, $\tau_{o}^{i}$ is not always given. In fact, $\tau_{o}^{i} \geq D_{q}^{i}+\Delta D_{q}^{i}$, where $\Delta D_{q}^{i}$ is a constant period of time allowing train to depart from the platform (to the exit). Thus in a view of station, function (5-1) is based on the arrival routes, while function (5-2) concerns all arrival and departure routes.

\subsubsection{Formulation in FIFO strategy}

The following formulation is based on this specific condition: the route (including the platform) for each train is fixed and the train orders are locally determined in the strategy of First-In First-Out (FIFO). That is, the train appearing earlier in the scope will be earlier routed. Under this precondition, no decision variables are actually to be determined. 
The function (5-1) or (5-2) are used to estimate the minimum arrival delay or minimum exit delay. And function (5-1) is subject to (5-3), (5-5), (5-7), (5-11) and (5$12)$, while function (5-2) is subject to (5-3) to (5-12).

$$
\begin{aligned}
& t_{s_{2}}^{i} \geq t_{s_{1}}^{i}+B_{s_{1} s_{2}}^{i}, \quad s_{1}, s_{2} \in R_{r}^{i}, i \in T S_{q} \\
& t_{o}^{i} \geq t_{s}^{i}+B_{s o}^{i}, \text { s is the last segment before } i \text { exits from station } q, i \in T S_{q} \\
& z_{q}^{i} \geq a_{q}^{i}-A_{q}^{i}, \quad i \in T S_{q} \\
& d_{q}^{i} \geq D_{q}^{i}, \quad i \in T S_{q} \\
& a_{q}^{i} \geq t_{s_{k}}^{i}+C^{i}, \quad s_{k} \in R_{r}^{i}, k \in K^{i}, i \in T S_{q} \\
& t_{s_{k}+1}^{i} \geq d_{q}^{i}, \quad S_{k}+1 \in R_{r}^{i}, k \in K^{i}, i \in T S_{q} \\
& d_{q}^{i} \geq a_{q}^{i}+W_{q}^{i}, \quad i \in T S_{q} \\
& t_{s_{k}+1}^{i} \geq t_{s_{k}}^{i}+B_{s_{k}\left(s_{k}+1\right)}^{i}+C^{i}+W_{q}^{i}, \quad s_{k}, s_{k}+1 \in R_{r}^{i}, k \in K^{i}, i \in T S_{q} \\
& t_{s}^{i_{2}} \geq t_{s}^{i_{1}}+C^{i_{1}}+\Delta f^{i_{1} i_{2}}, s \in\left(R_{r_{1}}^{i_{1}} \cap R_{r_{2}}^{i_{2}}\right), i_{1}, i_{2} \in T S_{q} \text { and } i_{2} \text { is after } i_{1} \\
& a_{q}^{i}, z_{q}^{i}, t_{s}^{i} \geq 0
\end{aligned}
$$

Constraints (5-3) and (5-4) generally specify that it takes at least the minimum running time for a train to pass each segment where train running time means the period between head-in and head-out of the train. Only when the train enters the platform segment an additional running time of train length is considered, in constraints (5-7) and (5-10). Not only the factor of train length, but also the arrival time, departure time, and wait time are restricted in constraints (5-6) to (5-10) when a segment is platform. In particular, the platform $s_{k}, k \in K^{i}$ is definite for each train. Segment $\left(s_{k}+1\right)$ here indicates the next one after platform segment $l_{k}$ in train travel direction, rather than the absolute index with one unit bigger than $s_{k} . W_{q}^{i}$ is the minimum wait time for train $i$ at station $q$. Constraints (5-5) and (5-12) imply the possibilities of recorded train delay, i.e. $z_{q}^{i}$ will be positive and take effect only when $a_{q}^{i}>A_{q}^{i}$, otherwise the train arrives early or on time and there will be no delay to record ( $z_{q}^{i}$ equals zero).

Constraint (5-11) specifies the headway requirement when a couple of trains are successive and travelling in the same direction, which is composite by the additional train-length time of the preceding train and a minimum following interval $\Delta f^{i_{1} i_{2}}$ (depending on the types of train). That is, the subsequent train can only enters the same segment as the preceding train does when the actual headway is not less than the minimum one. However, it does not mean it is impossible for the successive trains to be on the same segment, e.g. if the segment is long enough. The actual order of the successive trains may differ from that in schedule. 
With this formulation, the only manner to prevent a potential route conflict is letting the later coming train reduce the speed or wait before the conflicting route until the route is available for it. However, the cost of this strategy is that it may directly increase the total delay of trains and spread subsequent conflict to posterior trains.

\subsubsection{Formulation with changeable train order}

The formulation above is generated with fixed route distribution for each train and stochastic train orders (only depending on the first appearing orders) at joint point. It reduces the complexity of calculation, as well as the flexibility to reduce total delay or to solve a routing conflict. In this formulation we take the strategy that the train orders at a joint point are allowed to change, although the train routes are fixed as planned.

Firstly, we consider the joint point in a pair of specific routes, as Figure 5-3 shows, trains $i_{1}$ and $i_{2}$ come from different tracks; and they have to take routes $r_{1}$ and $r_{2}$ respectively to pass the same segment $s$. In the case (a) the two trains are separate again to distinct tracks after the joint point, while in the case (b) the double trains have to use the same track successively from the joint point. In general, if we let the following segment of joint point $s$ in route $r_{1}$ be $s_{1}$ and that of route $r_{2}$ be $s_{2}$ then the joint point in (a) and (b) can be described as $\left\{s, s_{1}\right\} \in R_{r_{1}}^{i_{1}},\left\{s, s_{2}\right\} \in R_{r_{2}}^{i_{2}}, s_{1} \neq s_{2}$ and $\left\{s, s_{1}\right\} \in$ $R_{r_{1}}^{i_{1}},\left\{s, s_{2}\right\} \in R_{r_{2}}^{i_{2}}, s_{1}=s_{2}$, respectively.

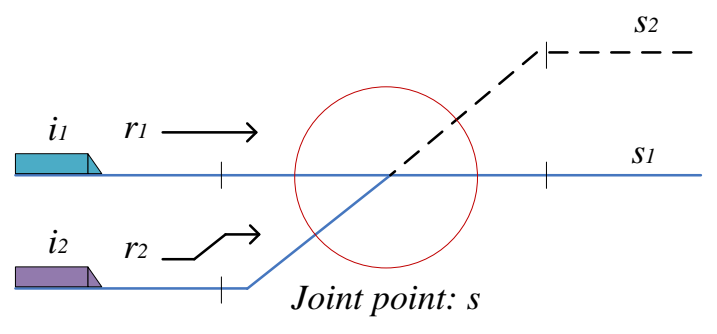

(a)

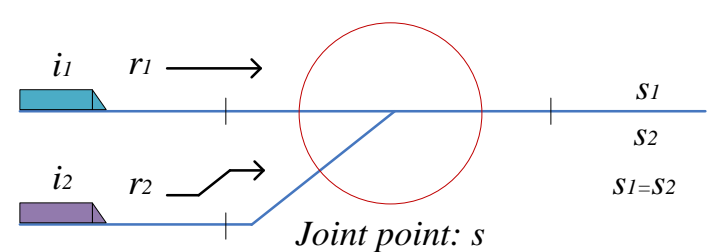

(b)

Figure 5-3 Joint point for trains

A type of binary variable $\alpha_{S}^{i_{1} i_{2}}$ is defined to represent the order of two trains $i_{1}$ and $i_{2}$ to pass the same segment (joint point) $s, s \in R_{r_{1}}^{i_{1}}$ and $s \in R_{r_{2}}^{i_{2}}$.

$$
\alpha_{s}^{i_{1} i_{2}}= \begin{cases}1, & \text { if train } i_{1} \text { uses segment } s \text { before train } i_{2} \\ 0, & \text { otherwise }\end{cases}
$$

A large constant $M$ is used to formulate the constraints of binary variables. For situation (a): constraint (5-13a) specifies that train $i_{2}$ is not allowed to enter the joint segment until the tail of train $i_{1}$ has left from it if train $i_{1}$ is going to pass the joint point before train $i_{2}\left(\alpha_{s}^{i_{1} i_{2}}=1\right)$; otherwise, constraint (5-14a) substitutes to ensure the entering time of train $i_{1}$ not earlier than the tail clearing time of train $i_{2}$. 


$$
\begin{aligned}
& t_{s}^{i_{2}} \geq t_{s_{1}}^{i_{1}}+C^{i_{1}}-M\left(1-\alpha_{s}^{i_{1} i_{2}}\right), \quad s_{1} \neq s_{2} \\
& t_{s}^{i_{1}} \geq t_{s_{2}}^{i_{2}}+C^{i_{2}}-M \alpha_{s}^{i_{1} i_{2}}, \quad s_{1} \neq s_{2}
\end{aligned}
$$

Similar constraints (5-13b) and (5-14b) are formulated for the situation (b).

$$
\begin{aligned}
& t_{s}^{i_{2}} \geq t_{s_{1}}^{i_{1}}+C^{i_{1}}+\Delta f^{i_{1} i_{2}}-B_{s s_{2}}^{i_{2}}-M\left(1-\alpha_{s}^{i_{1} i_{2}}\right), \quad s_{1}=s_{2} \\
& t_{s}^{i_{1}} \geq t_{s_{2}}^{i_{2}}+C^{i_{2}}+\Delta f^{i_{2} i_{1}}-B_{s s_{1}}^{i_{1}}-M \alpha_{s}^{i_{1} i_{2}}, \quad s_{1}=s_{2}
\end{aligned}
$$

For above inequalities, $s, s_{1} \in R_{r_{1}}^{i_{1}}, s, s_{2} \in R_{r_{2}}^{i_{2}}, i_{1}, i_{2} \in T S_{q}$. The minimum following interval is represented as $\Delta f^{i_{1} i_{2}}$ when the train sequence is $\left\{i_{1}, i_{2}\right\}$ and $\Delta f^{i_{2} i_{1}}$ when the sequence is $\left\{i_{2}, i_{1}\right\}$. That means not only the factor of train length is in restriction, but also the safety margin between the consecutive trains after they join to the same track is in consideration in constraints (5-13b) and (5-14b). In fact once the train sequence at the joint point is decided (either $\left\{i_{1}, i_{2}\right\}$ or $\left\{i_{2}, i_{1}\right\}$ ) in situation (b), the involved trains must satisfy the minimum headway constraint as that shows in (5-11).

$$
\alpha_{s}^{i_{1} i_{2}} \in\{0,1\}, \quad s \in R_{r_{1}}^{i_{1}}, s \in R_{r_{2}}^{i_{2}}, i_{1}, i_{2} \in T S_{q}
$$

With this formulation, train order is possible to be swapped at a joint point. In any of the following situations, it may be suitable to apply the formulation:

- If a potential route conflict contains any joint point;

- If a potential route conflict is farther than the joint point from the current position of conflicting trains (i.e. the trains will pass earlier on the joint point).

If the train order obtained via this formulation is not the same as the original arriving order, the early arriving train also has to reduce the speed and wait until another train passes by. In this case, a control signal or a dynamic speed restriction message is necessary before the joint point.

In real problems there may be more than one pair of trains involved at the same time whose orders are to be decided at several joint points. Then for each pair of trains at a single joint point a specific decision variable can be defined.

\subsubsection{Formulation with train route changeable}

To be more flexible, we further consider the scenario that a local train route (including the platform) can be different from that in a plan. That is, the strategy adopted is the train route on spot is allowed to change or to select from its possible route set when it has more than one possible route. And in this case long route of train is considered.

We define a long route of train is the feasible short path for a train from the specific entry of the station to the correspondingly specific exit, usually consisting of the arrival 
part and departure part (arrival route and departure route or pass route). Platform constraints are taken into account as well if any platform is included in a long route. When there are at least two long routes feasible for a train to realise all its planned operations, one and only one of them will be selected as the actual train route.

For example in Figure 5-4, train $i_{1}$ is planned to enter the station by entry $e_{1}$ and leave by entry (exit) $e_{2}$ while train $i_{2}$ is the opposite. When train $i_{1}$ is at the entry $e_{1}$ there are three long routes in its travel direction and two of them $\left\{R_{r_{1}}, R_{r_{2}}\right\}$ are feasible for train $i_{1}$, i.e. $R^{i_{1}}=\left\{R_{r_{1}}, R_{r_{2}}\right\}$. Similarly, among the three long routes in the direction of train $i_{2}$, exists the feasible route set $R^{i_{2}}=\left\{R_{r_{4}}, R_{r_{6}}\right\}$. In this way the actual route of train is flexible. But only one route for each train can be distributed at a time and all routes for different trains should not be conflicting.

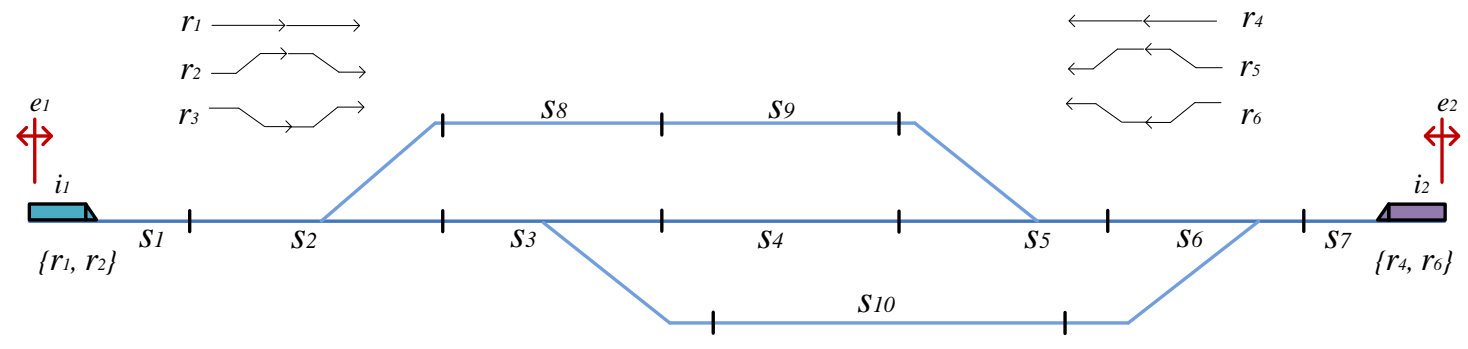

Figure 5-4 Long routes for trains

So another type of binary variable $\beta_{r}^{i}$ is defined to mark whether a train $i$ is to use long route $r, R_{r} \in R^{i}, R^{i}$ is the set of feasible routes for train $i$.

$$
\beta_{r}^{i}= \begin{cases}1, & \text { if train } i \text { is distributed to use route } r \\ 0, & \text { otherwise }\end{cases}
$$

It is noted that the feasible route set $R^{i}$ is dynamic, changing with the position of train and the availability of infrastructure. Based on this route set, constraint (5-16) ensures that there is only one long route being selected for each train. Let $I_{r}$ be the set of incompatible long routes in opposite direction of route $r, u \in I_{r}$. Then constraint (5-17) specifies that incompatible routes for a pair of trains $i_{1}, i_{2}$ travelling in opposite directions are forbidden to set at the same time. For example in Figure 5-4, route $r_{1}$ and route $r_{4}$ should not be distributed to $i_{1}$ and $i_{2}$ meanwhile.

$$
\begin{aligned}
& \sum_{R_{r} \in R^{i}} \beta_{r}^{i}=1, i \in T S_{q} \\
& \sum_{u \in I_{r}} \beta_{u}^{i_{2}}+\beta_{r}^{i_{1}} \leq 1, r \in R^{i_{1}}, u \in R^{i_{2}}, u \in I_{r}, i_{1}, i_{2} \in T S_{q}
\end{aligned}
$$

The train route decision further influences the covered segments including platform, the entering times and even the train orders at joint points. Constraints (5-3), (5-10) and (5-11) can be overwritten by (5-18), (5-19) and (5-20), respectively, with the route variable $\beta_{r}^{i}$ taken into account. 
$t_{s_{2}}^{i} \geq t_{s_{1}}^{i}+B_{s_{1} s_{2}}^{i}-M\left(1-\beta_{r}^{i}\right), \quad s_{1}, s_{2} \in R_{r}^{i}, i \in T S_{q}$

$t_{s_{k}+1}^{i} \geq t_{s_{k}}^{i}+B_{s_{k}\left(s_{k}+1\right)}^{i}+C^{i}+W_{q}^{i}-M\left(1-\beta_{r}^{i}\right), \quad s_{k}, s_{k+1} \in R_{r}^{i}, k \in K^{i}, i \in T S_{q}$

$t_{s}^{i_{2}} \geq t_{s}^{i_{1}}+C^{i_{1}}+\Delta f^{i_{1} i_{2}}-M\left(1-\beta_{r_{1}}^{i_{1}}\right)-M\left(1-\beta_{r_{2}}^{i_{2}}\right), s \in\left(R_{r_{1}}^{i_{1}} \cap R_{r_{2}}^{i_{2}}\right), i_{1}, i_{2} \in$ $T S_{q}, i_{2}$ after $i_{1}$

Moreover, the joint point as well depends on the routes that each involved train has taken. If the train orders are allowed to change at a joint point, constraints $(5-13 a, b)$ and $(5-14 \mathrm{a}, \mathrm{b})$ may as well have the extended versions $(5-21 \mathrm{a}, \mathrm{b})$ and $(5-22 \mathrm{a}, \mathrm{b})$, where $s, s_{1} \in R_{r_{1}}^{i_{1}}, s, s_{2} \in R_{r_{2}}^{i_{2}}, i_{1}, i_{2} \in T S_{q}$.

$t_{s}^{i_{2}} \geq t_{s}^{i_{1}}+C^{i_{1}}-M\left(1-\alpha_{s}^{i_{1} i_{2}}\right)-M\left(1-\beta_{r_{1}}^{i_{1}}\right)-M\left(1-\beta_{r_{2}}^{i_{2}}\right), \quad s_{1} \neq s_{2}$

$t_{s}^{i_{1}} \geq t_{s_{2}}^{i_{2}}+C^{i_{2}}-M \alpha_{s}^{i_{1} i_{2}}-M\left(1-\beta_{r_{1}}^{i_{1}}\right)-M\left(1-\beta_{r_{2}}^{i_{2}}\right), \quad s_{1} \neq s_{2}$

$t_{s}^{i_{2}} \geq t_{s_{1}}^{i_{1}}+C^{i_{1}}+\Delta f^{i_{1} i_{2}}-M\left(1-\alpha_{s}^{i_{1} i_{2}}\right)-M\left(1-\beta_{r_{1}}^{i_{1}}\right)-M\left(1-\beta_{r_{2}}^{i_{2}}\right), s_{1}=s_{2}$

$t_{s}^{i_{1}} \geq t_{s_{2}}^{i_{2}}+C^{i_{2}}+\Delta f^{i_{2} i_{1}}-M \alpha_{s}^{i_{1} i_{2}}-M\left(1-\beta_{r_{1}}^{i_{1}}\right)-M\left(1-\beta_{r_{2}}^{i_{2}}\right), s_{1}=s_{2}$

$\beta_{r}^{i} \in\{0,1\}, R_{r} \in R^{i}, i \in T S_{q}$

Not all constraints are always required. With the executing of train routes, variables are gradually determined and the margin of local adjustment is on the decrease until new trains enter the station scope.

\subsubsection{Operation time adjustment}

Operation time adjustment also contributes to facilitate the local routing realisation. However, since the limitation of speed in station area, we can find (from Figure 3-6) that the waiting time at platform is the only flexible time parameter among all the operation times inside the station. There can be a margin between planned waiting time and the minimum waiting time. Figure 5-5 shows an illustration of the time margin.

This margin can usually be exploited in the local routing optimisation. For example, inequalities (5-9), (5-10) and (5-19) have taken this margin into account. 


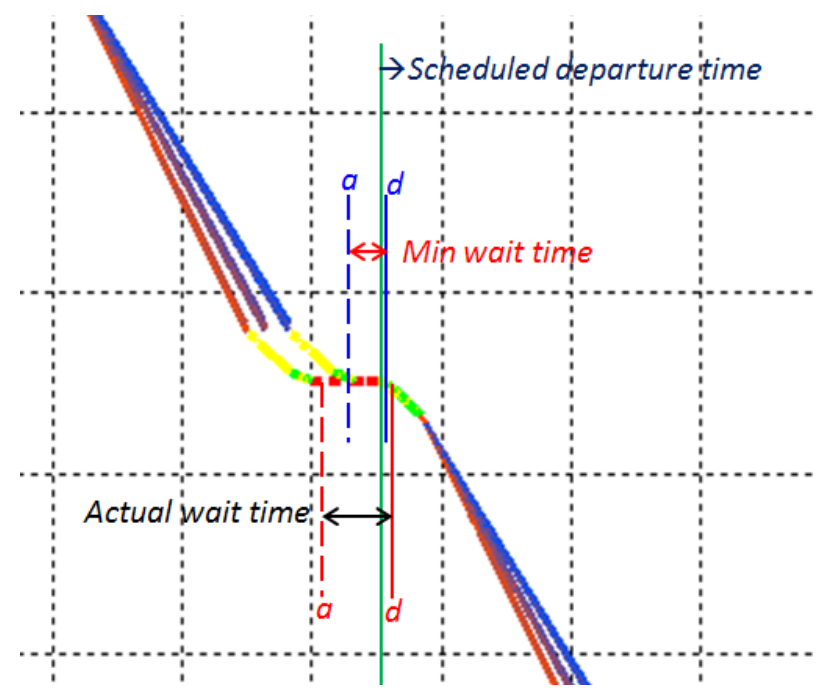

Figure 5-5 Illustration of the margin between planned wait time and the required value of minimum wait time

\subsubsection{Dynamic solution}

Generally, the conflict is a temporary phenomenon that integrates train route distribution, train order scheme, actual train kinetic characteristic and control mode, and infrastructure states at that given time, etc. As a consequence, solutions of conflict based on the state variables should not keep unique or constant. In other words, what a dispatcher or a dispatching support system has to find out is not only a static solution but a series of dynamic solutions for local routing and rerouting.

First, in Table 5-1 we appoint a classification of solution according to the concrete strategy adopted in particular cases.

Table 5-1 Solution levels and corresponding formulations

\begin{tabular}{cllcl}
\hline Level & Strategy & Variables & $\begin{array}{c}\text { Objective } \\
\text { functions }\end{array}$ & Constraints \\
\hline $\mathbf{1}$ & $\begin{array}{l}\text { Train routes fixed, } \\
\text { train orders at joint } \\
\text { points fixed }\end{array}$ & $t_{s}^{i}, t_{o}^{i}, a_{q}^{i}, d_{q}^{i}, z_{q}^{i}$ & $(5-1) /(5-2)$ & $(5-3)$ to $(5-12)$ \\
$\mathbf{2}$ & $\begin{array}{l}\text { Train routes fixed, } \\
\text { train orders flexible }\end{array}$ & $t_{s}^{i}, t_{o}^{i}, a_{q}^{i}, d_{q}^{i}, z_{q}^{i}, \alpha_{s}^{i_{1} i_{2}}$ & $(5-1) /(5-2)$ & $\begin{array}{l}(5-3) \text { to }(5-12), \\
(5-13 a) \text { to }(5-15)\end{array}$ \\
$\mathbf{3}$ & $\begin{array}{l}\text { Train routes } \\
\text { flexible, train } \\
\text { orders fixed }\end{array}$ & $t_{s}^{i}, t_{o}^{i}, a_{q}^{i}, d_{q}^{i}, z_{q}^{i}, \beta_{r}^{i}$ & $(5-1) /(5-2)$ & $\begin{array}{l}(5-4) \text { to (5-9), (5-12), } \\
(5-16) \text { to }(5-20),(5-23)\end{array}$ \\
$\mathbf{4}$ & $\begin{array}{l}\text { Train routes } \\
\text { flexible, train } \\
\text { orders flexible }\end{array}$ & $t_{s}^{i}, t_{o}^{i}, a_{q}^{i}, d_{q}^{i}, z_{q}^{i}, \alpha_{s}^{i_{1} i_{2}, \beta_{r}^{i}} \quad(5-1) /(5-2)$ & $\begin{array}{l}(5-4) \text { to }(5-9),(5-12), \\
(5-16) \text { to }(5-23)\end{array}$ \\
\hline
\end{tabular}

Different combinations of objective function, variables and constraints expressed above can be conditionally applied to work at different scenarios. In many cases, 
strategies may appear in other forms. Other rules can be taken into consideration as well, such as

- delayed trains have lower priority than those punctual;

- or high speed trains have to be set prior to the ones with lower speed, etc.

Actually, they indicate that the involved trains have the determined train orders if they are in the queue of any joint point. But it should be note that control signals or speed restriction messages are controllable for related joint points. Otherwise, the train order at joint points depends on the route reservation order.

In practice, if the operation rules are allowed we can further consider the following objectives: i) to minimise the total number of delayed trains, ii) or to minimise the total number of trains that have to be adjusted. But ultimately, the first objective is to solve the potential route conflicts in advance.

Then the dynamic procedure can be executed by periodically updating the input data, handling of current problem with updated information and updating the output. Input data includes trains and their performances, scheduled times and routes, actual train positions and speeds, arriving times of train at key points, segment and route states, operation strategies, latest solution and cumulative delay or other conflict information if there is. In some cases, there is not always new effective information in the input data, the handling behaviour can be event-driven. Particularly, when some train enters the scope its pending variables and constraints will be add to the formulation. Operation objective and local strategies finally determine the solution level and adopted formulation. The outcome of calculation during each period is a feasible solution of unknown variables in current situation, including route distribution for each train, train orders at some key points and corresponding entering times, as well as an estimated minimised total delay if the solution is accept. Periodically, some results also can be part of the input data at next step.

Figure 5-6 shows the pseudocode of the algorithm of dynamic solution of local routing problem. It is assumed that computation time at each step should be less than the updating period of data collection. 


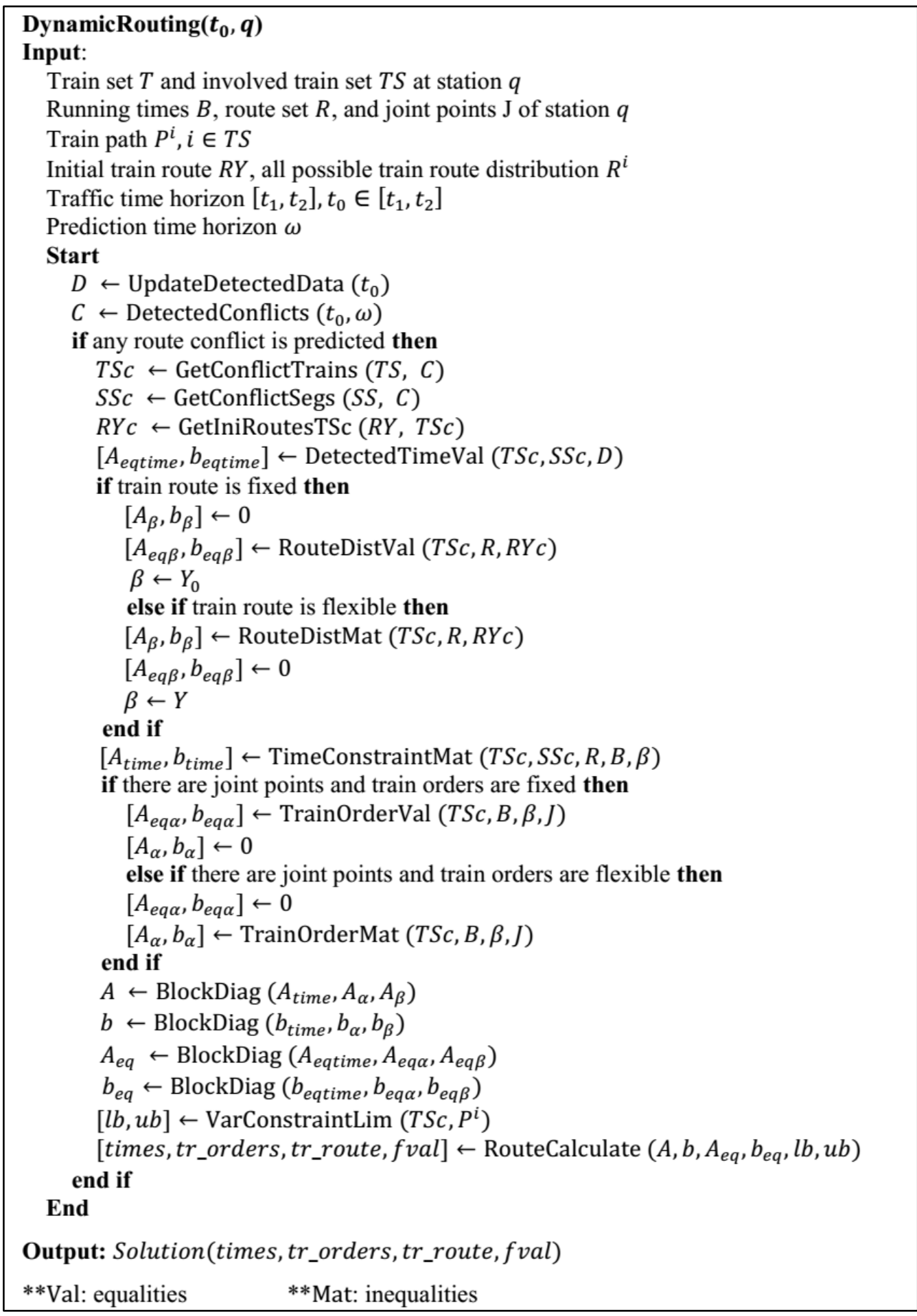

Figure 5-6 Pseudocode of dynamic solution of local routing and rerouting

\subsection{Rescheduling}

In the situation that all the local routing efforts to solve any route conflict are not efficient in controlling the other deviations (e.g. increasing delays and more frequent 
train speed variations), rescheduling measures that are applicable to the solution in a wider scope are considered.

The margin between planned waiting time and the minimum value of required waiting time is usually reserved for any adjustment in the local routing optimisation. It is kept in the rescheduling procedure, so that the local routing approaches remain feasible after rescheduling. Thus, the rescheduling approach mainly lies in the exploitation of the amply time gap existing in the flexible domain 'B12' and 'B23' that is shown in Figure 4-2.

\subsubsection{Train speed profile}

There are always a series of speed profiles that are selectable for the train under different control modes and line characteristics (e.g. curve, slope, static speed restrictions, etc.). As described in 4.1.1, for a particular train with scheduled times, still more than one speed profile can be derived from its planned train path. These speed profiles grouped by section (between two stations) can be numbered (ID) for selection. Each profile with its ID is related with a series of train control operations and statistical train running time on this section. For example, Figure 5-7 illustrates a cluster of possible train trajectories with different speed profiles.

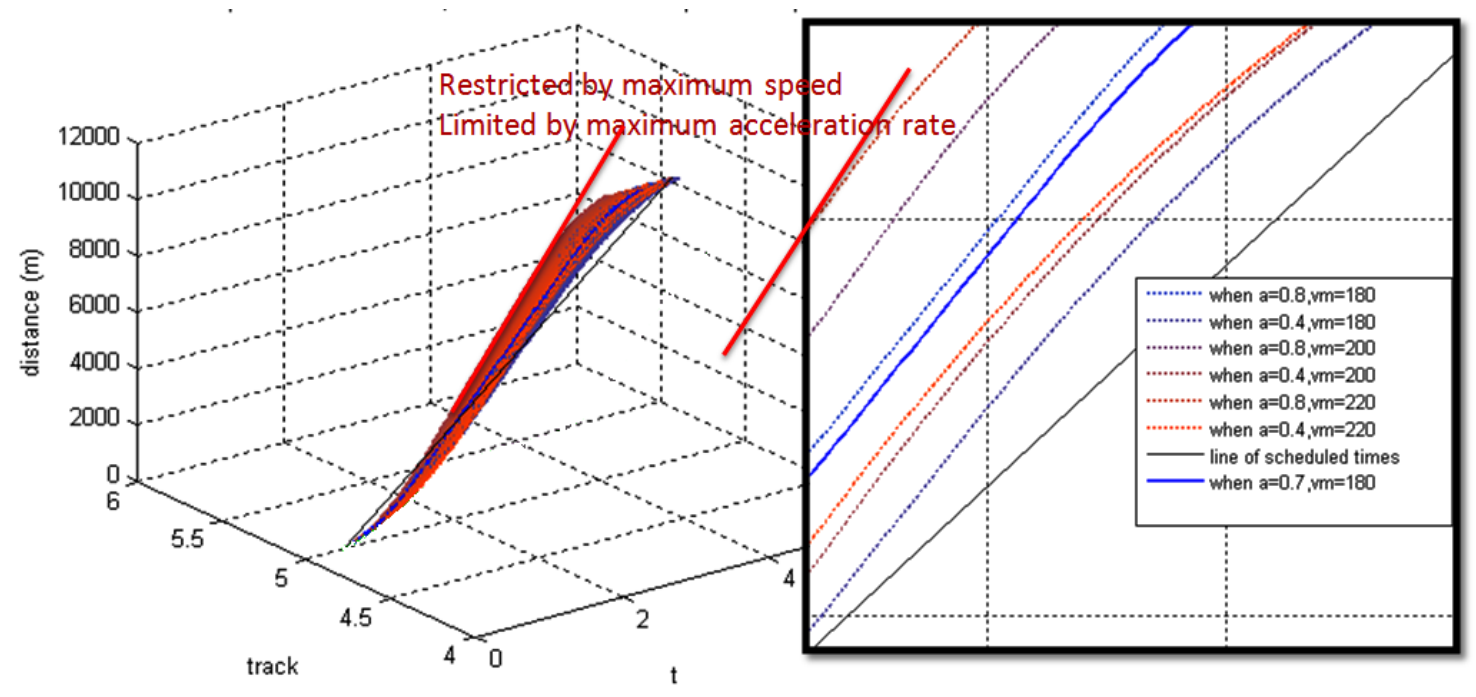

Figure 5-7 Illustration of possible train trajectories with different speed profiles

For a particular section that connects the stations $q_{1}$ and $q_{2}$ and a particular train (type) $i$, we use $\gamma_{q_{1}, q_{2}}^{i}$ to denote the corresponding running time to the selected speed profile. And the selected speed profile will be the updated $P^{i}{ }_{-} V(x)$.

Speed variation may affect the real-time headway restriction which is also related with the infrastructure as well as the signalling system. The headway distance at any moment for the successive trains is still restricted by the minimum headway restriction $h\left(t, v_{1}, v_{2}\right)$. The intervals (headway times) in rescheduling, denoted as $\tilde{I}^{i_{1}, i_{2}}$, may equal or vary from the original ones. But they shall satisfy the most restrictive interval value, 
i.e. the minimum interval value for successive trains to run with (the highest speed) profile and minimum running time $\left(Q_{q_{1}, q_{2}}^{i}\right.$, defined in Table 3-4). Updated (selected) speed profile and headway restrictions will be used in the posterior conflict detection procedure.

In the rescheduling problem, the empirical speed profile is not changeable while a train has set out from the station. It can be selectable from the next non-station section. However, the dynamic train speed may be adjusted immediately in order to avoid a headway conflict (Cx20) without exceeding the most restrictive speed profile (further discussion in 7.2).

\subsubsection{Variable scope rescheduling}

If the conflicts in an area are not well solved via local routing and rerouting, the conflicting area is firstly estimated, indicated by the number of stations and the number of involved trains. Rather than all line and network, the scope to be considered in rescheduling is about $300 \%$ of the conflicting area. For example, we can describe a conflict area as "a station with 4 trains", and by triple, the problem size in rescheduling is " 3 stations with around 12 trains". The extended stations are symmetrically located in upstream and downstream directions of the conflicting area. And the considered trains are the ones that are inside the conflicting area and that are going to enter the area. The multiple can be determined as an input parameter, e.g. here it is $300 \%$, and that might be $500 \%$ in another case. And the exact number of trains can also be decided in specific situations, e.g. the number of trains involved in a 3-station area may be 10 or 14, or other values.

Supposing that $n_{s t}$ stations and $n_{t r}$ trains are involved in a concrete rescheduling problem, where $n_{s t} \leq N_{s t}, n_{t r} \leq N_{t r}$. Let $T S$ be the set of trains and $E S$ be the set of stations. Let the following (5-24) be one of the objective functions in rescheduling.

$$
\min \sum_{q \in S S} \sum_{i \in T S}\left(\widetilde{A_{q}^{i}}-A_{q}^{i}\right), T S \subset T, E S \subset E
$$

For each train $i \in T S$, the rescheduling is subject to the following constraints if corresponding conditions are satisfied.

$$
\begin{aligned}
& \widetilde{D_{q}^{l}}-D_{q}^{i} \geq 0, q \in E S, \text { where } d_{q}^{i} \text { has not happend } \\
& \widetilde{D_{q}^{l}}-\widetilde{A_{q}^{l}} \geq D_{q}^{i}-A_{q}^{i}, q \in E S, \text { where } a_{q}^{i} \text { and } d_{q}^{i} \text { at least one is unknown } \\
& \widetilde{A_{q_{1}}^{l}}-\widetilde{D_{q}^{l}} \geq \gamma_{q, q_{1}}^{i}, q, q_{1} \in E S, q_{1} \text { is the next station for } i \text { after station } q
\end{aligned}
$$

Basically, constraint (5-25) specifies the requirement of not departing earlier than the original departure time that is often published officially. Then, on the base of satisfying the minimum waiting time restriction (3-3), constraint (5-26) also keeps waiting time margin for local routing. Constraint (5-27) reserves sufficient running time for the train 
by using the selected speed profile and $\gamma_{q, q_{1}}^{i}$ is the running time from station $q$ to $q_{1}$ on the selected speed profile. It is similar to the restriction (3-4), or they are the same when the most restrictive speed profile is selected and $\exists \gamma_{q, q_{1}}^{i}=Q_{q_{1}, q_{2}}^{i}$. However, to take effect in conflict resolution, the running time of the selected speed profile is often less than the one in the original schedule.

Constraint (5-28) specifies the headway requirement for a pair of successive trains in the same direction, where $i_{1}, i_{2} \in T S$ and $i_{2}$ succeeds $i_{1}$.

$$
\widetilde{D_{q}^{i_{2}}}-\widetilde{D_{q}^{i_{1}}} \geq \widetilde{I}^{i_{1}, i_{2}}, \quad q \in E S, \text { where } d_{q}^{i_{1}} \text { and } d_{q}^{i_{2}} \text { are unknown }
$$

If there are any trains scheduled for (passenger or cargo) connection purpose in the original schedule, it is also considered in the rescheduling. For example, if trains $i_{1}, i_{2} \in T S$ are planned to connect at station $q$ (on different platforms), constraints (5-29) and (5-30) consider the connection time (transfer time or operation time $\Delta c^{i_{1}, i_{2}}$ and $\Delta c^{i_{2}, i_{1}}$ ) from one train to another. According to the requirement, (5-29) and (5-30) can be used separately or at the same time.

$$
\begin{aligned}
& \widetilde{D_{q}^{i_{1}}}-\widetilde{A_{q}^{i_{2}}} \geq \Delta c^{i_{1}, i_{2}}, \quad q \in E S, \text { if connecting } i_{2} \text { to } i_{1} \\
& \widetilde{D_{q}^{i_{2}}}-\widetilde{A_{q}^{i_{1}}} \geq \Delta c^{i_{2}, i_{1}}, \quad q \in E S, \text { if connecting } i_{1} \text { to } i_{2}
\end{aligned}
$$

If a pair of trains $i_{1}, i_{2} \in T S$ have to stop at the same platform at station $q$, similar to the constraints at joint point, constraint (5-31) describes the time restriction to avoid train $i_{2}$ from using the platform before train $i_{1}$ departs from it $\left(\Delta f^{i_{1}, i_{2}}\right.$ is the minimum following interval), and (5-32) restricts the times when the trains use the platform in reverse order (the minimum interval is $\Delta f^{i_{2}, i_{1}}$ ). Binary variable $\delta_{q}^{i_{1}, i_{2}}$ is the decision indicator of the order trains $i_{1}$ and $i_{2}$ to use the platform.

$$
\delta_{q}^{i_{1}, i_{2}}= \begin{cases}1, & \text { if train } i_{1} \text { uses the platform before train } i_{2} \\ 0, & \text { otherwise }\end{cases}
$$

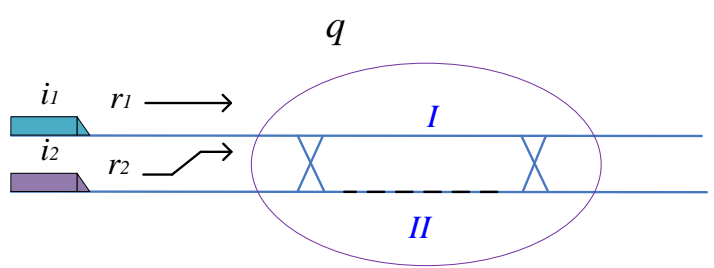

(a)

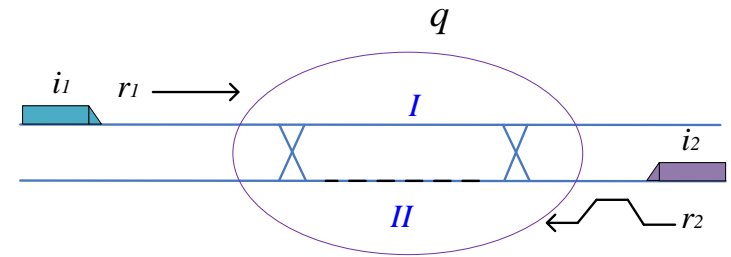

(b)

Figure 5-8 In the case: two trains have to use the same platform (I) inside station

$$
\widetilde{A_{q}^{i_{2}}}-\widetilde{D_{q}^{i_{1}}} \geq \Delta f^{i_{1}, i_{2}}-M\left(1-\delta_{q}^{i_{1}, i_{2}}\right), \quad q \in E S
$$




$$
\begin{aligned}
& \widetilde{A_{q}^{i_{1}}}-\widetilde{D_{q}^{i_{2}}} \geq \Delta f^{i_{2}, i_{1}}-M \delta_{q}^{i_{1}, i_{2}}, \quad q \in E S \\
& \delta_{q}^{i_{1}, i_{2}} \in\{0,1\}, \quad q \in E S
\end{aligned}
$$

Once the binary value of $\delta_{q}^{i_{1}, i_{2}}$ after the rescheduling is different from that of the original schedule, the train order is swapped. In case the train orders are required to remain the same as in the original schedule, the indicator $\delta_{q}^{i_{1}, i_{2}}$ can be configured as a definite value.

If train $i_{1}$ from station $q_{1}$ to the next station $q_{2}$ has to use the same track with trains $i_{2}$ from station $q_{2}$ to $q_{1}$, as Figure 5-9 shows, we can further introduce another type of binary variable $\vartheta_{q_{1}, q_{2}}^{i_{1}, i_{2}}$ to indicate the decision of the order to use the open track. This type of decision variable will be restricted by (5-34) and (5-35).

$$
\vartheta_{q_{1}, q_{2}}^{i_{1}, i_{2}}= \begin{cases}1, & \text { if train } i_{1} \text { from station } q_{1} \text { uses the track first } \\ 0, & \text { otherwise }\end{cases}
$$

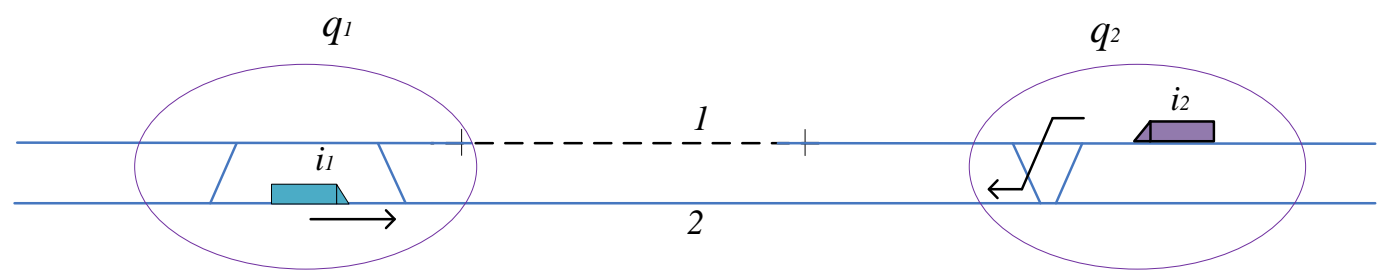

Figure 5-9 In the case: two trains have to use the same track (2) from opposite directions

$$
\begin{aligned}
& \widetilde{D_{q_{2}}^{i_{2}}}-\widetilde{A_{q_{2}}^{i_{1}}} \geq \gamma_{q_{1}, q_{2}}^{i_{1}}-M\left(1-\vartheta_{q_{1}, q_{2}}^{i_{1}, i_{2}}\right), \quad q_{1}, q_{2} \in E S \\
& \widetilde{D_{q_{1}}^{i_{1}}}-\widetilde{A_{q_{1}}^{i_{2}}} \geq \gamma_{q_{2}, q_{1}}^{i_{2}}-M \vartheta_{q_{1}, q_{2}}^{i_{1}, i_{2}}, \quad q_{1}, q_{2} \in E S \\
& \vartheta_{q_{1}, q_{2}}^{i_{1}, i_{2}} \in\{0,1\}, \quad q_{1}, q_{2} \in E S
\end{aligned}
$$

Finally, the facts (as initial conditions) should be taken into account, such as:

$$
\begin{aligned}
& \widetilde{A_{q}^{l}}=a_{q}^{i}, \quad q \in E S \text {, if } a_{q}^{i} \text { is known } \\
& \widetilde{D_{q}^{l}}=d_{q}^{i}, \quad q \in E S \text {, if } d_{q}^{i} \text { is known } \\
& \widetilde{A_{q}^{l}}, \widetilde{D_{q}^{l}} \geq t_{0}, \quad q \in E S \text {, if } a_{q}^{i}, d_{q}^{i} \text { are unknown }
\end{aligned}
$$

(5-39) requires that all rescheduled times are not earlier than the moment $t_{0}$ when the rescheduling is carrying out. 
The rescheduling approach follows above formulation and outputs a new (partial) schedule for the considered trains and stations. Since the scope of the rescheduling is variable, depending on the predicted conflicting area at the moment and the configured parameter (e.g. about 300\% illustrated at the beginning of this section), it is called variable scope rescheduling. Figure 5-10 shows the pseudocode of this rescheduling procedure.

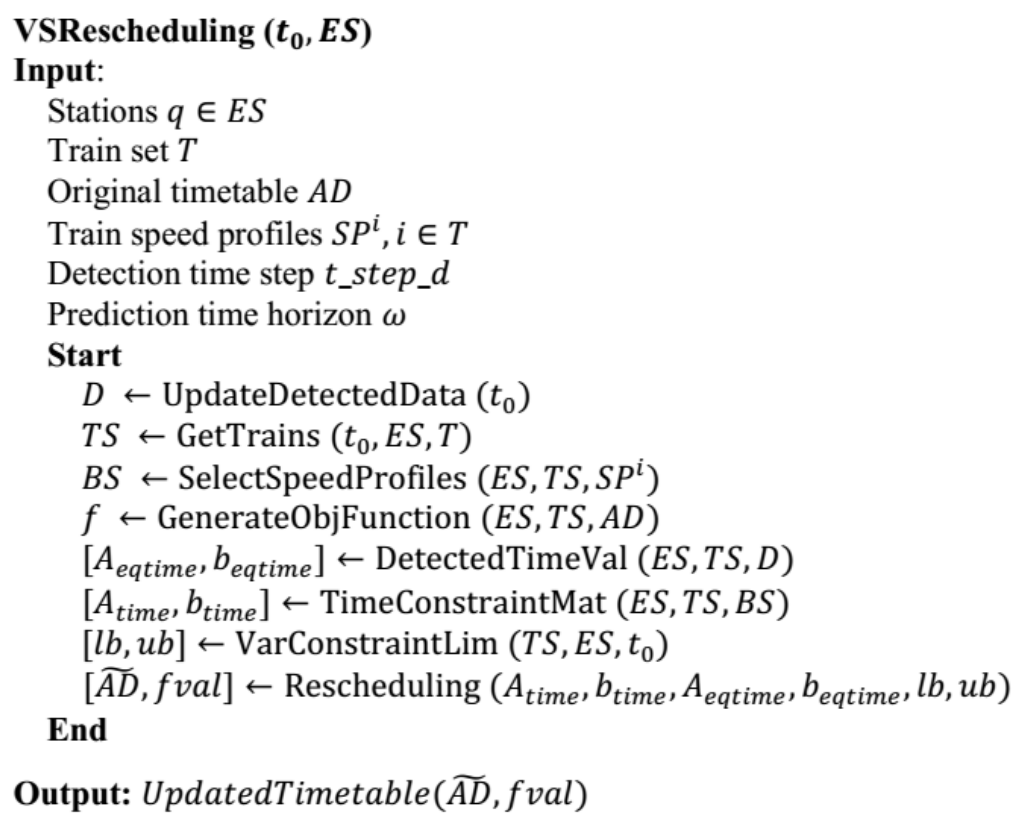

Figure 5-10 Pseudocode of variable scope rescheduling

\subsection{Integration in resolution}

Local routing and rescheduling approaches divide the conflict resolution by different conflict situations; at the same time, these approaches cooperate with each other in composing general conflict solutions.

\subsubsection{Integrated resolution}

The two layers of methods suggested in Figure 5-1 are integrated in the resolution procedure, whose pseudocode is expressed in Figure 5-11.

When a route conflict is detected, local routing approach is employed first and the predictive traffic prediction with suggested routing adjustment will be simulated. In this way the feasibility of routing proposal is self-evaluated before it is implemented. Only when the local routing approach fails to resolve the conflict efficiently or a human command of rescheduling is received, the rescheduling procedure starts. In this case, the rescheduled timetable (partially for the considered scope) will be a mandatory solution that all related entities will update the new timetable as the reference of their subsequent 
operations. Nevertheless, the rescheduling formulation remains the flexibility for further routing and rerouting in the updated timetable.

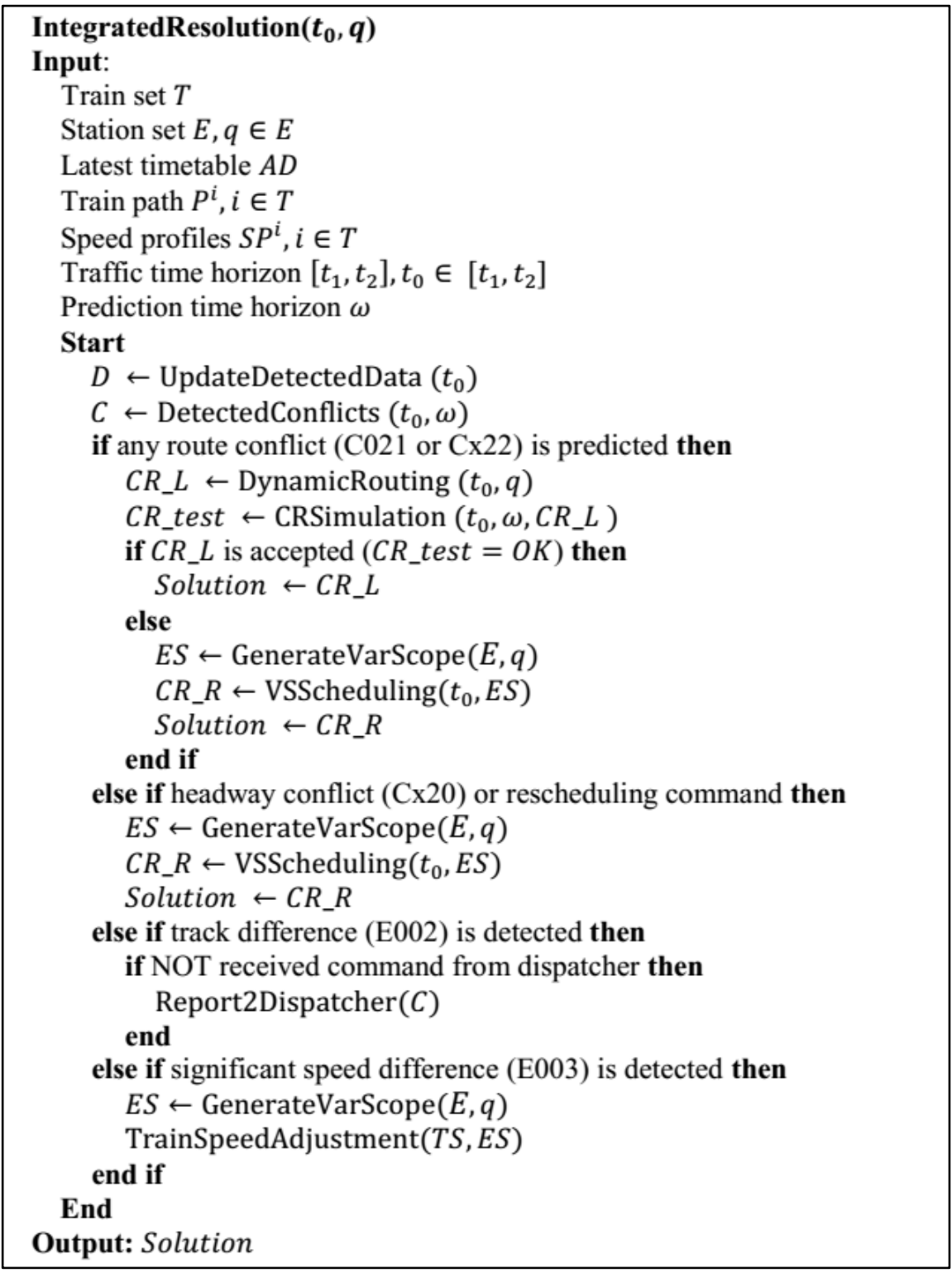

Figure 5-11 Pseudocode of the integrated approach for conflict resolution

\subsubsection{Resolved conflicts}

Corresponding to the conflicts and early warnings received from the conflict detection procedure in 4.3.2, Table 5-2 shows the resolution approaches for each potential conflict or conflict sign. The priority of each possible resolution approach in the list for a same conflict (conflict sign) is from highest to lowest, for example, even when obvious delay is detected, the first option is to keep observation and conflict 
detection, and rescheduling will be considered only when other messages are obtained in conflict detection or other traffic controller gives command to implement the rescheduling method.

Table 5-2 Predicted conflicts and corresponding resolution approaches

\begin{tabular}{l|l|l}
\hline Message code & Description & Resolution \\
\hline E001 & Significant delay & $\begin{array}{l}\text { a. Observation and conflict estimation } \\
\text { b. Rescheduling }\end{array}$ \\
\hline E002 & Track difference & $\begin{array}{l}\text { a. Report to higher controller } \\
\text { b. Rescheduling }\end{array}$ \\
\hline $\mathbf{E 0 0 3}$ & Speed deviation & $\begin{array}{l}\text { a. Dynamic speed adjustment } \\
\text { b. Rescheduling }\end{array}$ \\
\hline $\mathbf{E x 0 4}$ & Headway risk & $\begin{array}{l}\text { a. } \text { Rescheduling } \\
\text { b. Dynamic speed adjustment }\end{array}$ \\
\hline $\mathbf{C x 2 0}$ & Headway conflict & a. Local routing \\
\hline $\mathbf{C 0 2 1}$ & $A 1$ route conflict & b. Rescheduling \\
\hline Cx22 & $I C$ route conflict &
\end{tabular}

Table 5-2 shows that the local routing approach is only applied when route conflicts are detected, while the rescheduling approach seems a feasible way to solve any conflicts or deal with all conflict signs. Theoretically, any approach following the rescheduling formulation is directly against conflicting routes, so the predicted route conflicts, which are not efficiently resolved by local routing, can be avoided naturally by rescheduling. However, in many cases, even being minimised, the very cost of rescheduling is a general delay (to the original schedule) for a wide group of trains. Thus, as mentioned above, the rescheduling approach is not always called immediately except that the situation is against headway restrictions. By selecting proper speed profiles, estimated headway conflicts are removed in the new schedule.

Still two more conditions are mentioned in rescheduling:

i) the authority to access into a higher level of traffic control by considering more sections and involving more trains;

ii) more time in advance to realise the rescheduling and to update the new schedule for any related entities that are obligated or recommended to know the new schedule.

\subsection{Conclusions}

In this chapter two layers of resolution approaches are presented. The routing approach in station area is proposed as a principle solution of route conflicts. And the rescheduling approach is mainly in charge of the prevention of headway hazards or delay propagation in a wider area than a station.

Some advantages of the methodology are summarised as follows. 
- Each layer can function independently or synthetically. However, the integration of both layer allows the predicted conflicts to be resolved in a more systemic and flexible way. The approach on each layer cannot always be suitable in all occasions. For the transport with fixed train ID where the resource and service is pre-distributed to fixed users (e.g. in long distance railway system), local routing and dynamic speed adjustment may be utilised more frequently; for short-journey and periodic transport where the frequency draws more attention than the punctuality and the headway restrictions come to a bigger challenge (e.g. in metro, subway system), rescheduling can be the solution more efficient. But the combination makes the methodology gain adaptability to different systems.

- New dispatching strategies can be added to the local routing formulations.

- The constraints expressed in the rescheduling formulation are still appropriate in other methods. Other than MILP, other rescheduling algorithms can be introduced to the same layer for conflict resolution. For example, we can refer to those approaches stated in literature (Samà et al., 2016b; Wang et al., 2016).

- The size of rescheduling is controllable (in this chapter, $300 \%$ of the conflict size is exampled). It allows the rescheduling problem agile enough to be computed at low time cost. Besides, it is flexible to vary the scope (vary the solution neighbourhood) of the problem while no satisfying solution is found in the specified scope.

- Unless extremely serious interruption (for accident or other reasons), local adjustment and variable scope rescheduling may control the propagation of conflict at an early stage.

There are also some further issues to be considered. For example, it is not discussed how to ensure conflict-free at global level (in the whole network). More time and efforts will be required in macroscopic rescheduling. However, a global rescheduled timetable does not ensure conflict-free in future traffic due to the stochastic characteristics of conflict. It can be a topic to study the favourable size of scope in timetable rescheduling. The advantages and disadvantages of each approach also depend on the complexity of the railway network and the designed flexibility of the original timetable. 

Chapter 6

\section{CDR support system}

An agent-based CDR support system is modular designed for railway traffic management. 


\section{Chapter 6 CDR support system}

Namely, a CDR support system is a functional entity (software or device) designed to aid the traffic management system to predict any potential conflict and to take automatic measures or provide advice for conflict resolution. CDR support system can serve as one of the active safety systems for railway traffic management.

In this chapter an agent-based method is proposed to develop the CDR support system, named D-Agent. Hansen (2009) stated that efficient traffic management support systems must be able to simulate the effects of different dispatching measures and support traffic controllers by frequently updating the actual timetable and ranking the dispatching options according to their expected performance. We introduce the agentbased approach for the CDR support system for the following considerations.

(a) A D-Agent aims at supporting traffic optimisation at station (and section) level, where the local schedule (a part of the schedule of the entire network) can be the reference of all its behaviour.

(b) The agent can be flexibly equipped with a variety of knowledge and methods. It provides the foundation for simulating and testing the possible effects of different measures. Owing to its internal reasoning mechanism and evaluation function, it can furthermore generate advice in decision-making.

(c) Significantly, a well alive agent is dynamic. Based on the accurate data of actual train positions and speeds, conventional events and potential conflicts can be predicted in advance. Thus corresponding conflict resolution and advice can take effect in subsequent operations.

(d) Furthermore, historical data and experience may help the agent to update its knowledge and skill bases. Meanwhile, it is possible for the agent to introduce new knowledge or modify its existing CDR methods by learning.

(e) A D-Agent can work in a wider environment where the interaction and communication with other agents is always in process. It allows the data collection and solution implement or advice output to be dynamic.

\subsection{Agent-based approach in railway dispatching}

Davidsson et al. (2005) surveyed the agent-based approaches in transportation and traffic management. The conclusion they drew was that agent-based approaches seemed suitable for the transport domain from a logistical perspective, yet that more deployed systems still needed to be developed to verify the applicability on practical level. As a fact, four areas had been classified to apply agent-based modelling in (Bonabeau, 2002). They were areas of flows, markets, organizations and diffusion. Traffic was included in the area of flows, where an early agent-based traffic simulation software package (TRANSIMS) was introduced. TRANSIMS could simulate a virtual metropolitan region with individuals in the region, their activities, and the transportation 
infrastructure. In road transportation, dynamic modelling of traffic disturbance and corresponding regulation with agent-based techniques were proposed and tested by using real data of Brussels bus network (Balbo and Pinson, 2005, 2010).

Although only a few surveyed publications on agent-based approaches for transport are exactly concerning the rail systems, there is still some work having well started. For example, in (Törnquist and Davidsson, 2002) the authors described a multi-agent system approach to handle the train delays, with a railway network simulator based on traditional simulation techniques and a negotiation simulator of the decision making actors based on the multi-agent-system (MAS) technique; and Corman and D'Ariano (2008) introduced a novel multi-agent architecture for designing proactive operational traffic management systems where train drivers, dispatchers and network controllers were viewed as autonomous agents in the environment of railway network; the authors in (Lu and Zhang, 2009) adopted a multi-agent approach to solve the subway operation adjustment where train agents were constructed to be automatic operated, and they stated that the proposed approach was feasible and possible to extend dynamically.

In an survey of dynamic scheduling approaches (Ouelhadj and Petrovic, 2009), the MAS technique was highlighted for its promising performance in complex and dynamic environments. Two main multi-agent architectures (autonomous and mediator architectures) for dynamic scheduling were concluded to simulate behaviour of different agent roles. Concerning the real-time agent-based solutions for railway rescheduling and routing, the authors in (Narayanaswami and Rangaraj, 2015; Proença and Oliveira, 2004) proposed the two architectures of multi-agent system coincidentally with two main layers. In (Proença and Oliveira, 2004) a MAS architecture with a control subsystem aiming at providing secure and efficient routing for all trains and preventing conflicting situations and another sub-system containing all learning and supervisor agents was presented. In (Narayanaswami and Rangaraj, 2015), the so-called regulating (or rescheduling) layer is incorporated by supervisor, train, station and a new type of agent: auctioneer agent, where an auctioneer agent identifies the conflicting trains for a particular disruption and remains active till that disruption is resolved; the learning layer has also been extended with learner and facilitator agents, where the facilitator agent provides the selected rule to the system after learning. Globally, the supervisor agent is active all the time on both layers. Narayanaswami and Rangaraj (2015) also evaluated the agent approach by comparing the experimental results with CPLEX solver. It concluded that CPLEX solver can work quite fast when the problem size is small however it requires a very large computational time in some (more difficult) problem instances; whereas all problem instances can be solved in almost uniform time via the multi-agent approach. This significant feature of agent approach promisingly indicates its potential capacity to solve the problem in a larger sized and more complex railway network. 
Continuing with but also distinguishing from the previous work, the D-Agent presented in this chapter focuses on the internal design with the conflict detection and resolution methods proposed in previous chapters.

\subsection{D-Agent modelling}

The D-Agent is modularly designed. Before the description of each module, the environment of a D-Agent and its individual functional structure are introduced.

Rather than replacing an agent of infrastructure or station, a D-Agent aims at assisting in traffic dispatching at station and region level. One D-Agent is designed for a certain station area (or a region containing several sections if necessary). Therefore, the environmental factors of the agent include corresponding infrastructure and signalling facilities, the trains in the scope, and human dispatchers. In addition, the neighbourhood (with the same type of agents) also affects the behaviour of an individual agent. With regard to the manual operation of local dispatcher, we define two modes of D-Agent: in full autonomous mode (mode A), the local dispatcher is liberated from the routine work and the D-Agent handles all the jobs and makes all the decisions; in supporting mode (mode S), relatively being more conservative, the D-Agent deals with all information in advance and merely proposes possible solutions for the local dispatcher when the problems occur and the dispatcher makes the final decisions. Thus the commands from local dispatcher can also be considered as an environmental factor of D-Agent when it works in mode $\mathrm{S}$.

Macal and North (2006) introduced the concepts and the general process of agentbased modelling and simulation. And still inspired by a general rational knowledge based agent modelling in (Freeman, 1990), Figure 6-1 specifies the individual structure of D-Agent. The structure of agent does not depend on its operating mode.

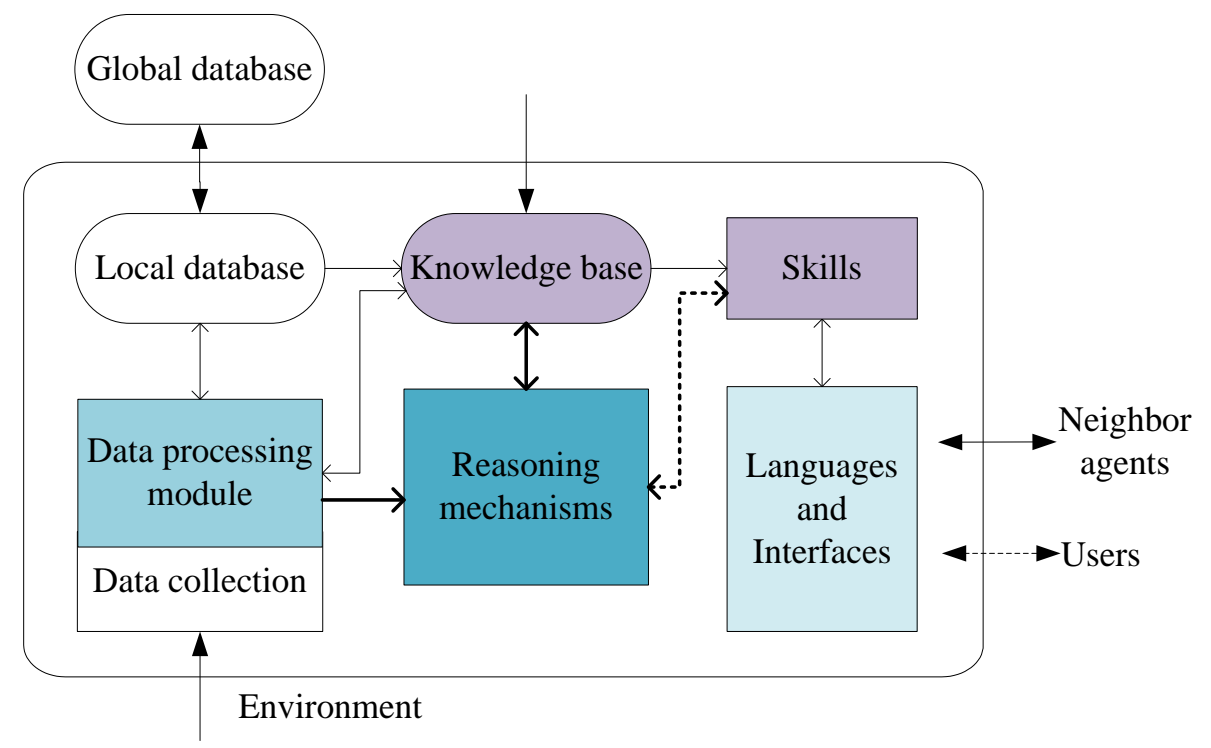

Figure 6-1 Basic modules of a D-Agent 
A typical D-Agent mainly has six basic modules: local database, private knowledge base (facts and rules), skill base, data processing module, reasoning mechanism and language applications (interfaces). The data processing module can also be considered as a separated skill of the D-Agent with two functions: i) predicting and detecting potential conflicts and conflict signs; ii) preparing the data in proper formats and accelerating the general procedure. In skill base, the approaches of conflict resolution are encapsulated as distinct skills that can be called directly if corresponding preconditions are satisfied.

\subsubsection{Database}

The public part of data in local database includes the information of railway network, train performances and the standard (original) timetable, etc. which is provided by the global database in Figure 6-1.

Meanwhile, the detailed infrastructure data, planned and actual train sequence according to the timetable, route table, etc. that are locally stored usually can be private or protected. Local data of the D-Agent is also grouped as static attributes and state variables, respectively. The state variables are continually collected and dynamically updated. Some historical data is also locally stored, such as empirical train speed profiles, route state (hash) table, etc.

D-Agent also stores the detected conflict records together with corresponding solutions that have been applied.

\subsubsection{Knowledge}

The knowledge base of the D-Agent can also be partly inherited from the agent class and partly learned by experience.

The inherited knowledge is like the congenital condition underlying the development of the agent (by itself) as well as the very important starting point in solving the problems encountered. For example, a rule list of 'shall do' and 'must not do', the map and facilities of the station along with the constraints, the pre-defined schedule and route table, the train list, etc. all can belong to this type of knowledge.

Being more flexible but not less crucial, the learned part provides increasingly new knowledge and promotes the agent to learn fresh skills to adapt the changes of the objective and the environment. For example, the agent may be inculcated the integrated message 'at station A, to reserve route 1 for train 02 when it enters segment 1 (is a feasible way)' from the recent operation records or directly controlling inputs by dispatchers, etc. Furthermore, in the long term the agent can gain new statistical knowledge by analysing the historical data from the database. The knowledge of train paths (and their static derivatives) and the empirical infrastructure state transition maps belongs to this category. 
Some common instances of knowledge are shown in Figure 6-2. Since the local data at each particular area is different, the knowledge of a D-Agent may as well vary from another's, i.e. one kind of knowledge of a D-Agent may not suitable for another.

\begin{tabular}{|c|c|}
\hline 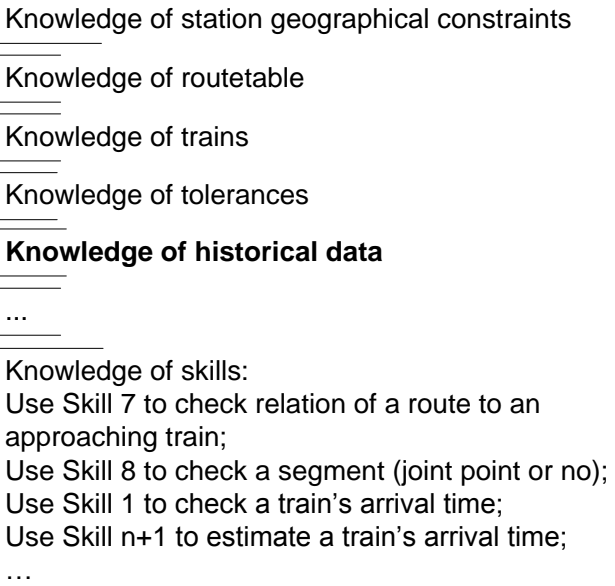 & $\begin{array}{l}\text { Skills: } \\
\text { Skill 1: Get arrival time; } \\
\text { Skill 2: Get departure time; } \\
\ldots \\
\text { Skill 7: Get scheduled route of a train; } \\
\text { Skill 8: Judge a segment a joint point; } \\
\text { Skill 9: Find routes related to a segment; } \\
\text { Skill 10: Estimate influenced trains; } \\
\ldots \\
\text { Skill } n+1 \text { : Estimate arrival time; } \\
\text { Skill } n+2: \text { Optimise train sequence; } \\
\text { Skill } n+3: \text { Optimise long routes; } \\
\text { Skill } n+4: \text { Record train sequence; } \\
\text { Skill } n+5: \text { Record long routes; } \\
\text { Skill } n+6: \text { ReSchedule timetable; } \\
\ldots\end{array}$ \\
\hline
\end{tabular}

Figure 6-2 Knowledge and skill fragments of the D-Agent

\subsubsection{Data processing}

All the data from the environment are firstly collected to the data processing module. There might be much more data than necessary being collected in its natural form (or several particular protocol formats but not concordant). Thus the processing module pre-processes the collected information by selecting the most related pieces of message, grouping and preparing filtered information in the best form for use. It initialises the variables and the constraints by extracting the original timetable, field topology, signalling logic and operation rules, etc. It also adjusts the objectives and updates the state variables of infrastructure, trains and the signalling system.

The models of train, infrastructure and timetable equip the data processing module. Besides, this module takes charge short period traffic prediction and conflict detection. Only the irregular traffic states and predictively conflicting situations will be further handled by the conflict resolution methods in other modules.

\subsubsection{Skills}

Figure 6-2 still shows a conceptual skill list of the D-Agent, where each method no matter simple or complicate is a skill, such as obtaining a train ID and its corresponding information, getting a route ID (usually a default route) according to the schedule and train ID, reserving segments according to the selected route ID, revoking a reserved route, reorder the train sequence, detecting delays, or calculate a new timetable (for limited area), etc. The D-Agent uses specific skills to complete some actions. 
The internal skills of the D-Agent are assorted into two groups: one can be directly manipulated by existing knowledge and the other are learned from the external world or from its experience. The first group is knowledge-based and related to the concrete controlled area, whilst the second is based on exotic algorithms and methods that the agent masters. Figure 6-2 also implies that the knowledge may be the guidance of the skills, while conversely the skills produce more experience and new rules. The procedure that an agent tries to apply a new method (skill), evaluates its performance, and finally gains new rules from the experience is an important way of learning.

It is assumed that the D-Agent has a total number of $n$ knowledge-based skills at the initial stage, and the skills indexed from $n+1$ are extended other than the existing knowledge of the D-Agent. These extended skills include the approaches of local routing, the measures of dynamic train speed adjustment and the approaches of regional rescheduling, and other feasible conflict resolution approaches (if any), etc.

\subsubsection{Reasoning mechanism}

The behaviour of an agent is dictated by its internal architecture, based on the state of itself and the changes that have taken place in the environment (Park and Sugumaran, 2005). So the reasoning mechanism of D-Agent is described in terms of the concrete logic of D-Agent's behaviour.

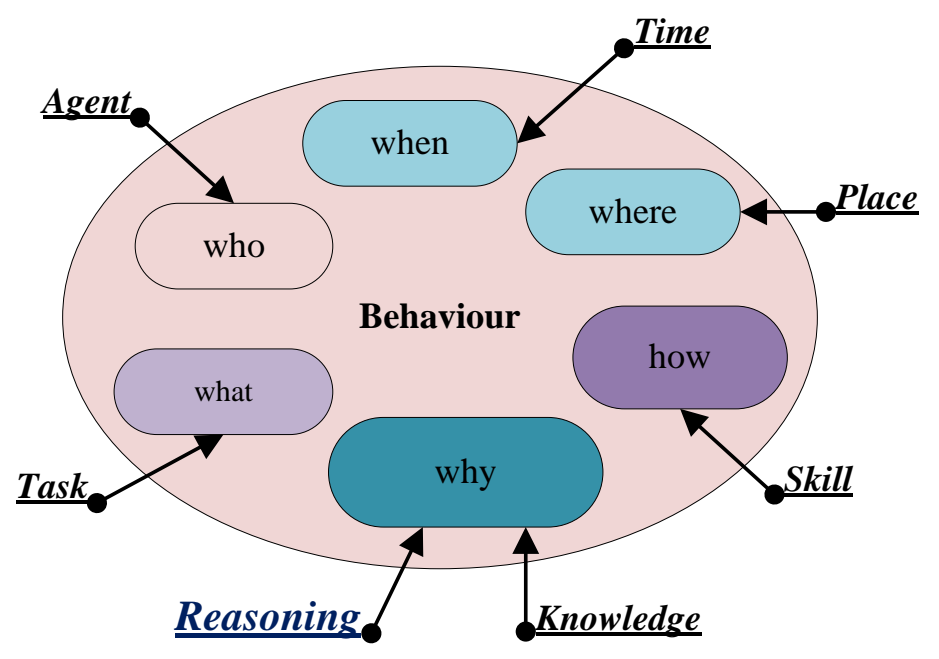

Figure 6-3 Elements of behaviour

Generally, certain behaviour of an agent can answer such questions in Figure 6-3, and conversely the elements - the particular agent, time and space, the task, knowledge or reasoning results and the skills used, are factored to simulate the behaviour of the agent. A task is an action that the D-Agent has to take in a specific situation, e.g. to make a judgement (whether there is a potential conflict or no), to make a decision (which route to assign to a particular train), or to work out a routing solution (in a conflict situation), etc. In Figure 6-3 the skills are applied to fulfil the task, and the knowledge provides the 
foundation for the agent to select a best (or good enough) skill. Exact time and location also stamp various cases and record important experience.

The reasoning process of the D-Agent works in background all the time. It analyses the data (from data processing module) and draws out a list of tasks. According to the analysis results, the reasoning process drives the agent to select knowledge and skills to fulfil the tasks in order. An influence diagram is used to represent the internal reasoning mechanism of the D-Agent in Figure 6-4.

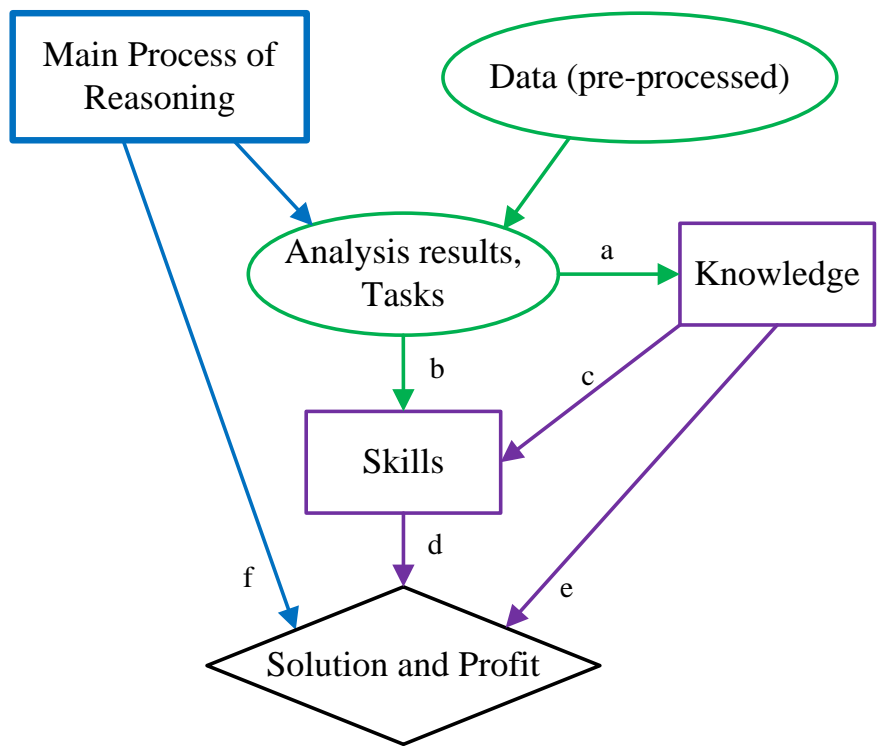

Figure 6-4 Influence diagram of D-Agent's reasoning mechanism

There are three types of nodes in the diagram: decision nodes (rectangles), information and chance nodes (ellipses) and utility node (diamond). The nodes directing to a rectangle are the critical factors that can directly influence the decision-making.

Significant relationships in this diagram include:

- Fundamentally, the reasoning module stimulates the other two decision nodes (the knowledge module and the skill module) by providing classified information, i.e. data analysis results and task information.

- On condition that the analysis results show a well-known case (edge a), the knowledge module decides the knowledge to use, including the suggested skill(s) as 'knowledge of skills' shown in Figure 6-2. In this case the knowledge module directly manipulates the skill module (edge c).

- Otherwise (edge b), when the feasible solution is beyond the D-Agent's knowledge, the skill module decides preferable skill(s) to adapt the unfamiliar situation rather than be guided by the knowledge module.

- The different decision nodes in the reasoning mechanism have the same target: to accomplish each task and get a solution for any problem that may occur. Yet the profit of each node is distinct from others'. For a skill module the contribution weight and preference weight of a particular skill may increase 
(edge d); new knowledge and experience may enrich the rule base (edge e); and the reasoning module may generate further decision (edge f), which finally represents the decision advice of the D-Agent to its neighbour agents and other users.

Reasoning and learning behaviour is basic intelligence of agent. Higher intelligence is the adaptability to the environment (Elofson et al., 1997). Detecting the potential conflicts from the traffic environment and seeking efficient approaches to avoid the conflicts belongs to the higher intelligence. However the realisation of the intelligence still relies on the cooperation with other systems in the same network.

\subsubsection{Communication}

A D-Agent exchanges information with the other agents in the entire environment, e.g. parallel D-Agents, centralized traffic control agents on upper layer, train and infrastructure agents (if existing) on lower layer, etc. It corresponds to the communication function, which in Figure 6-1 is the language module. In this thesis, the information of train movement, signalling system and infrastructure states are considered to be available for the D-Agent by data collection and processing.

Assume the agent knows all languages (protocols) used for the following interfaces:

- Between D-Agents: this interface is between two neighbour D-Agents (of neighbour area), by which messages about upstream and downstream trains and their departure times from one area (to another area) as well as delays (if any) are communicated; besides, the information of temporary abnormal situations is expected to be informed to the neighbours.

- D-Agent and human users: this is the GUI designed for the agent to work in mode S (supporting). Dynamic traffic analysis and possible advice will be presented to the human users (local dispatcher, if necessary as well centre dispatcher), in turn the interface imports the decisions and other commands input by the users. This also indicates the possibility to update the knowledge and skills of D-Agent by external factors.

- D-Agent and local traffic control system: this interface can be valid only if the mode of a D-Agent is autonomous and the latter gives authority to the D-Agent to deliver suggestions (note that although in this way, the agent is not able to control the traffic directly, but to influence the decision-making of the traffic control system). Similar to those from dispatchers, the actual control strategies adopted by the control system are informed to the D-Agent.

Some interfaces are optional for different D-Agents, depending on the actual traffic control system. By communication and cooperation with others, an individual D-Agent obtains a more comprehensive perspective to solve its local routing problem and furthermore contributes its effort to cope with variable scope rescheduling problem. 


\subsection{Learning}

Extended skills can be further self-evaluated by the D-Agent after being applied for numerous times.

Preference weight is a type of statistical parameter defined for each alternative solution adopted to fulfil a particular task. For example, with regard to the routing methods provided in 5.1, let $p_{r}$ be the preference weight of corresponding route $r$ applied in a task (for example, to find a long route in a route conflict), and let $c_{s}$ be the rate of changing orders of train pairs (i.e. different from the scheduled orders) at joint point $s$.

$$
\begin{aligned}
& p_{r}=\frac{\text { frequency of route } r \text { being assigned }}{\text { total number of times the task appears }} \\
& c_{s}=\frac{\text { frequency of train orders changed at joint point } s}{\text { total number of times to decide train orders at joint point } s}
\end{aligned}
$$

The total number of times that a certain task appears during a period of time is known and considered to be constant. It is affected by the width of time window used to observe. And the time window is also the memory span of the D-Agent for this particular task. Only some new records are uploaded to the knowledge base when this span is going to be closed. Each preference weight can be reset when its memory span restarts.

Preference weights are updated based on the dynamic traffic state that is the integration of train route distribution, train order scheme, actual train kinetic characteristic and control mode, and infrastructure states at that given time, etc. A local dispatcher or a D-Agent always has to find out dynamic solutions. Each dynamic solution can be carried out by a series of steps and each step is considered to be static in a certain period of time.

Subsequently, the dynamic procedure can be executed by periodically updating the input data, reasoning, selecting skill to obtain feasible solution, making decision or output timely advice, and updating the preference weights. This procedure is shown in Figure 6-5. Periodically, some results can be part of the input data at next cycle. Particularly, the preference weights may affect the future decisions for the same type of tasks. The skills having contributed to greater preference weights gain even greater possibilities to be applied in similar scenarios. The human decision of the dispatcher and the intervention of new skills can adjust the updating results of preference weights. 


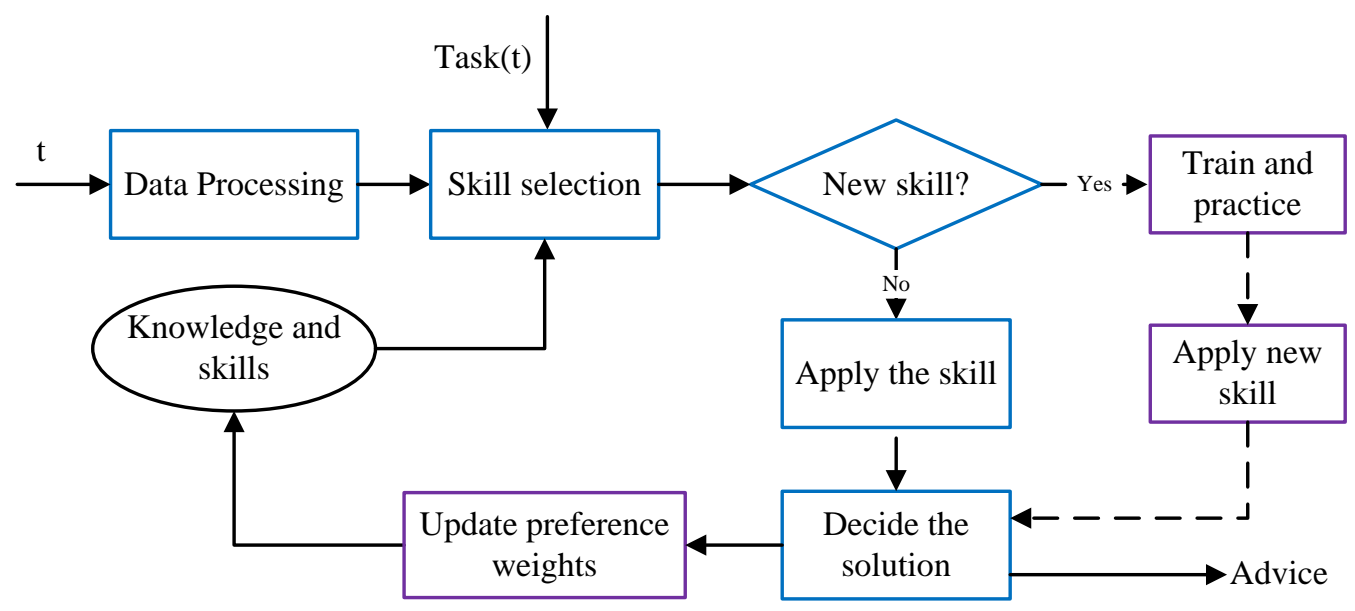

Figure 6-5 Dynamic update and learning skill of D-Agent

New preference weight arises when a new solution is adopted by the D-Agent, as well new experience can be added to the knowledge base. For example, the preference weights of conflict resolution by local routing approaches or by variable scope rescheduling approaches can be recorded. By the first trials and repetitive selfevaluation, the D-Agent learns new knowledge gradually from the extended skills.

\subsection{CDR decision support}

We take the following terminal station A as an instance to apply the proposed DAgent and analyse its behaviour.

\subsubsection{Case description}

Considering that a set of trains indexed $\{1,2, \ldots, 15\}$ are of the same train type, and all these trains are periodically planned, whose running direction is shown in Figure 6-6. The terminal station $\mathrm{A}$ has three feasible long routes $r_{1}, r_{2}, r_{3}$. According to the experience of local dispatchers, route $r_{1}$ alternating with route $r_{2}$ is a common preference for the shorter travel time compared with that of route $r_{3}$. Minimum stop time at platform for all trains is 30 time unit and the minimum time for a train to change the direction takes an extra 10 time unit. Besides, the train set and route table are accumulated as innate knowledge of the D-Agent for station A, as well as the information of segment elements in each route and corresponding running times. 


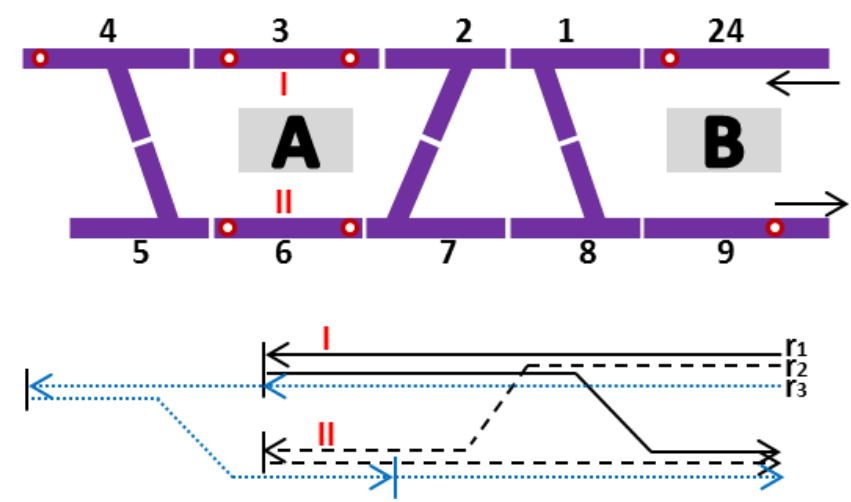

Figure 6-6 An illustration of terminal station A: in this station only 2 entries (entrance and exit connecting with station B), 8 segments (indicated by Arabic numerals) and maximum 2 tracks with platform (indicated by Roman numerals). Segments 1, 2, 4, 5, 7, 8 are switches, and in certain circumstances are joint points. There are 3 optional long routes for a train to approach, change the direction and depart for a new cycle.

Minimum stop time at platform for all trains is 30 time unit and the minimum time for a train to change the direction takes an extra 10 time unit. Besides, train set and route table are accumulated as basic knowledge of the D-Agent. The later contains information of segment elements of each route and corresponding running times.

The (current) situation to consider: the first train departs as normal from station B (to station A) whose departure time is referred as time 0 . All succeeding trains were scheduled to depart from station B at intervals of 120 time unit. However, for some reason the second train has to depart from station $\mathrm{B}$ with a certain delay $z_{B}^{2}$, causing that the intervals of involved trains $\{1,2,3,4\}$ are expected to shorten by 90 time unit at station A. Schedule for other trains remains unchanged. All operations are in a same memory span of the D-Agent, i.e. the preference weights will not be reset in this example. Table 6-1 and Table 6-2 show the updated tasks and related skills of the DAgent.

Table 6-1 Updated tasks of D-Agent (at station A)

\begin{tabular}{ll}
\hline Task ID & Description \\
\hline T.1 & $\begin{array}{l}\text { Prepare for train } 1 \text { to arrive at } 55 \text { (time unit) and departure at } 240 \text { (as } \\
\text { normal). }\end{array}$ \\
T.2 & Prepare for train 2 to arrive at 145 (time unit) and departure at 330. \\
T.3 & Prevent conflict between train 1 and 2. \\
T.4 & Prepare for train 3 to arrive at 235 (time unit) and departure at 420. \\
T.5 & Prevent conflict between train 1,2 and 3. \\
T.6 & Prepare for train 4 to arrive at 325 (time unit) and departure at 510. \\
T.7 & Prevent conflict between train 2,3 and 4. \\
T.8 & Detect potential conflicts. \\
\hline
\end{tabular}

Tasks 1, 2, 4 and 6 are of the same type yet they are all one-time tasks, and 3, 5, 7 are another type of tasks. Differing from the others, task 8 is durative and reduplicate. However, a local dispatcher may not have task 8 in his/her list. 
Table 6-2 Frequently used skills of the D-Agent

\begin{tabular}{ll}
\hline Skill ID & Description \\
\hline S.1 & Get scheduled route of a train \\
S.2 & Check the availability of a route \\
S.3 & Find possible route set for a same task \\
S.4 & Decide a route for a train \\
S.5 & Reserve a route for a train \\
S.6 & Get arrival time of a train \\
S.7 & Get departure time of a train \\
S.8 & Update involved train set \\
S.9 & Check conflict between related train pair \\
S.10 & Decide train orders \\
S.11 & Exchange information and commands with other internal modules of D- \\
& Agent \\
S.12 & Accept a new created (extended) skill (from external) \\
S.13 & Update skill list \\
S.14 & Update preference weights \\
S.15 & Reset preference weights \\
S.16 & Memory management \\
S.n+1 & Estimate arrival time of a train via MILP \\
S.n+2 & Decide train orders via MILP \\
S. $\boldsymbol{n}+3$ & Decide a route for a train via MILP \\
\hline
\end{tabular}

Skill 11 in Table 6-2 contains all internal information exchanges of the D-Agent. It gets the received messages from the communication module, seeks support from the knowledge base and reasoning mechanism, and sends commands to the communication module to generate specific messages.

Skills 4, 9 and 10 are initially based on the following rules in the knowledge module. It is assumed that these rules are followed by the dispatchers.

- Assign the shortest route to train $i$ if there are more feasible routes.

- Assign route $r_{1}$ to train $i$ if it is going to enter the station and track I is available at that moment.

- Assign route $r_{2}$ to train $i$ if it is going to enter the station and track II is available at that moment.

- If the actual interval between two trains $i_{1}$ and $i_{2}$ is less than 85 time unit, the train pair may be conflicting.

- Train orders remain as they are in the schedule.

For a D-Agent, new skills are only selectable when it is in training. As the extended skills are added, possible rules that might arise during the training are observed.

The D-Agent of station A is simulated as the initial delay of train 2 from station $\mathrm{B}\left(z_{B}^{2}\right)$ values $10,20,40,60,80,100,150$ and 200 time unit, applying the skills before and 
after the extension, respectively. For the purpose of comparison, the traffic simulation with human dispatcher's participation is also used for reference.

\subsubsection{Results and analysis}

The simulation results are analysed in three aspects.

First, behaviour of the D-Agent is observed in each single simulation. Figure 6-7 shows the D-Agent's behaviour streams, respectively, of the simulated D-Agent with and without extended skills. In the chart the initial delay of train 2 at station B values 60 time unit that the D-Agent of station A obtains from its neighbour D-Agent (of station B).

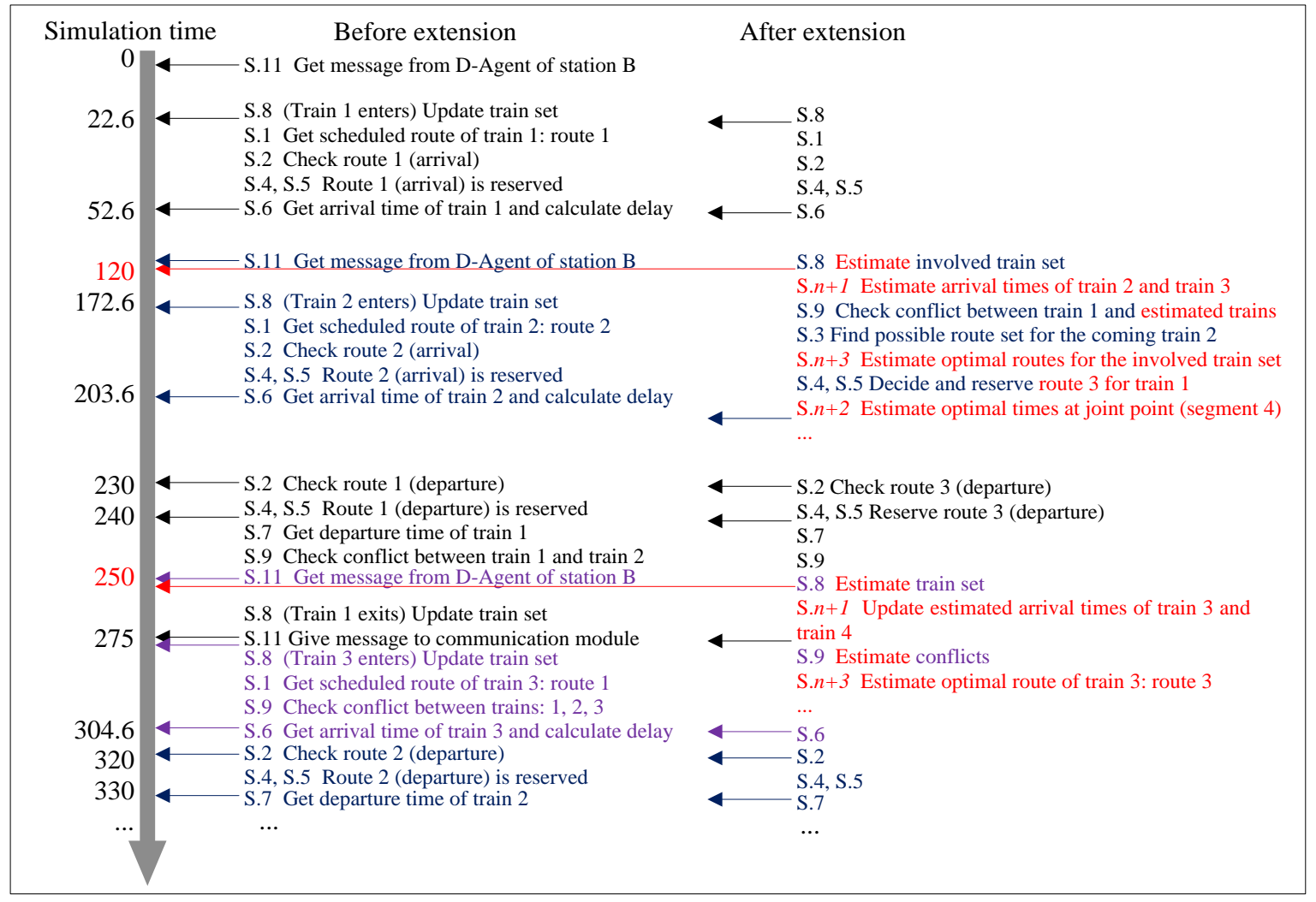

Figure 6-7 Behaviour streams of the D-Agent (when the initial delay of train 2 is 60 time unit)

From the behaviour streams, we can find: 1) the D-Agent can basically imitate a dispatcher to execute the definite tasks of local dispatching by utilizing its knowledge and skills; 2) moreover, the D-Agent with extended skills still attempts to estimate the upcoming situation (the trains approaching and their arrival times) and make an adjustment plan in advance by using the embedded formulations.

Then the dispatching results evaluated by total delay of trains (arrival and exit) are listed in Table 6-3. As reference, the total delays under the dispatching of a human operator are calculated via the objective functions (5-1) and (5-2) after simulation. The 
D-Agent without extended skills, which to some degree is a simulator of the human dispatcher, follows all the rules that a dispatcher has to follow. However, in the cases that the initial disturbances are slight (when $z_{B}^{2} \leq 60$ in the example) the D-Agent without extended skills does not seem as flexible as the dispatcher, e.g. it results much more total arrival delay than the dispatcher does when $z_{B}^{2}=10$. We ascribe it to the very rigid restrictions predefined for the D-Agent. Fortunately, that changes when the initial disturbance gets larger, and the D-Agent shows its advantage in more complicate calculation. In contrast, the D-Agent applying the extended skills raises the general controlling effect of the total arrival delay, even when the initial disturbances are not obvious. Nevertheless, the D-Agent applying the extended skills still seems to lack advantages when the total exit delay (objective function (5-2)) is considered. And the superiority of its distinct proposals from the dispatcher remains to be discussed.

It should be noted that the results of using extended skills are directly dependent on the methods we try to introduce to the agent, e.g. MILP routing in this example. In other words, the advantages of MILP approach may be delivered to the D-Agent, as well as the limitations. However, the D-Agent decides to use the skill or not in accordance with specific conditions. For example, in Table 6-3 the D-Agent may avoid using the extended skills when $z_{B}^{2}>100$. Furthermore, as the MILP formulations can be implanted, other approaches are also possible to be used to enrich the skill base so that the D-Agent can select the best fit skill to overcome certain shortcoming of other skills.

Table 6-3 Updated preference weights in simulation (partial results)

\begin{tabular}{|c|c|c|c|c|c|c|}
\hline$z_{B}^{2}$ & \multicolumn{2}{|c|}{ Human operator } & \multicolumn{2}{|c|}{ D-Agent without extended skills } & \multicolumn{2}{|c|}{ D-Agent applying extended skills } \\
\hline 10 & 21 & 0 & 148 & 0 & 7.6 & 0 \\
\hline 20 & 52 & 0 & 158 & 0 & 23.6 & 0 \\
\hline 40 & 114 & 0 & 178 & 0 & 78 & 0 \\
\hline 60 & 174 & 8 & 198 & 0 & 138 & 42 \\
\hline 80 & 234 & 88 & 218 & 0 & 198 & 102 \\
\hline 100 & 293 & 166 & 258 & 0 & 256.8 & 162 \\
\hline 150 & 461 & 96 & 408 & 73.6 & 408 & 312 \\
\hline 200 & 639 & 294 & 558 & 273.6 & 558 & 462 \\
\hline
\end{tabular}

The third aspect to analyse is the learning results of the D-Agent, including its new proposals. Since there are no trains has changed their orders in this example (i.e. $c_{S}$ remains 0), Table 6-4 shows only the route solution preference weights changing with the initial delays in simulation, where the dispatcher's preference is also referred to. From the preference weights we can observe that at the goal of 90 time unit's intervals the D-Agent starts to suggest route $3\left(p_{3}>0\right)$ for the subsequent trains to minimise the total arrival delay and exit delay at station A when the initial delay is around 10 to 60 time unit, which has never appeared in the initial rules (followed by the D-Agent before the skill extension or by the dispatcher where $p_{3}=0$ ). When the initial delay of train 2 values 100 time unit, alternating route 1 and route 2 as planned turns to be the preferable solution of the D-Agent (as it is expected). 
Table 6-4 Updated preference weights in simulation (partial results)

\begin{tabular}{|c|c|c|c|c|c|c|c|c|c|c|}
\hline \multirow{2}{*}{$z_{B}^{2}$} & \multirow{2}{*}{$\begin{array}{l}\text { Simulatio } \\
\text { n time }\end{array}$} & \multicolumn{3}{|c|}{ Dispatcher's preference } & \multicolumn{3}{|c|}{$\begin{array}{l}\text { D-Agent without extended } \\
\text { skills }\end{array}$} & \multicolumn{3}{|c|}{$\begin{array}{l}\text { D-Agent with extended } \\
\text { skills }\end{array}$} \\
\hline & & $p_{1}$ & $p_{2}$ & $p_{3}$ & $p_{1}$ & $p_{2}$ & $p_{3}$ & $p_{1}$ & $p_{2}$ & $p_{3}$ \\
\hline 10 & 0 & \multirow{4}{*}{0.5} & \multirow{4}{*}{0.5} & \multirow{4}{*}{0} & 0.5 & 0.5 & 0 & 0.5 & 0.5 & 0 \\
\hline 10 & 200 & & & & 0.5 & 0.5 & 0 & 0.5 & 0.45 & 0.05 \\
\hline 10 & 300 & & & & 0.5 & 0.5 & 0 & 0.45 & 0.45 & 0.1 \\
\hline 10 & 400 & & & & 0.5 & 0.5 & 0 & 0.45 & 0.4 & 0.15 \\
\hline 60 & 0 & \multirow{4}{*}{0.5} & \multirow{4}{*}{0.5} & \multirow{4}{*}{0} & 0.5 & 0.5 & 0 & 0.5 & 0.5 & 0 \\
\hline 60 & 200 & & & & 0.5 & 0.5 & 0 & 0.5 & 0.45 & 0.05 \\
\hline 60 & 300 & & & & 0.5 & 0.5 & 0 & 0.45 & 0.45 & 0.1 \\
\hline 60 & 400 & & & & 0.5 & 0.5 & 0 & 0.45 & 0.4 & 0.15 \\
\hline $\begin{array}{l}10 \\
0\end{array}$ & 0 & \multirow{5}{*}{0.5} & \multirow{5}{*}{0.5} & \multirow{5}{*}{0} & 0.5 & 0.5 & 0 & 0.5 & 0.5 & 0 \\
\hline 10 & 200 & & & & 0.5 & 0.5 & 0 & 0.5 & 0.5 & 0 \\
\hline 10 & 300 & & & & 05 & 05 & 0 & 05 & 05 & 0 \\
\hline 0 & 300 & & & & 0.5 & 0.5 & 0 & 0.3 & 0.0 & \\
\hline $\begin{array}{l}10 \\
0\end{array}$ & 400 & & & & 0.5 & 0.5 & 0 & 0.5 & 0.5 & 0 \\
\hline
\end{tabular}

Apart from the proposed solutions, we can further observe the underlying information of routing as the initial delay varies. Figure 6-8 shows the train arrival delays and exit delays depending on the initial disturbance of train 2 . The solid lines are the solutions with the original strategy to keep the original route distribution with routes 1 and 2 (strategy 1), and the dashed lines are solutions with route 3 that has not been planned (here we may call it strategy 2).
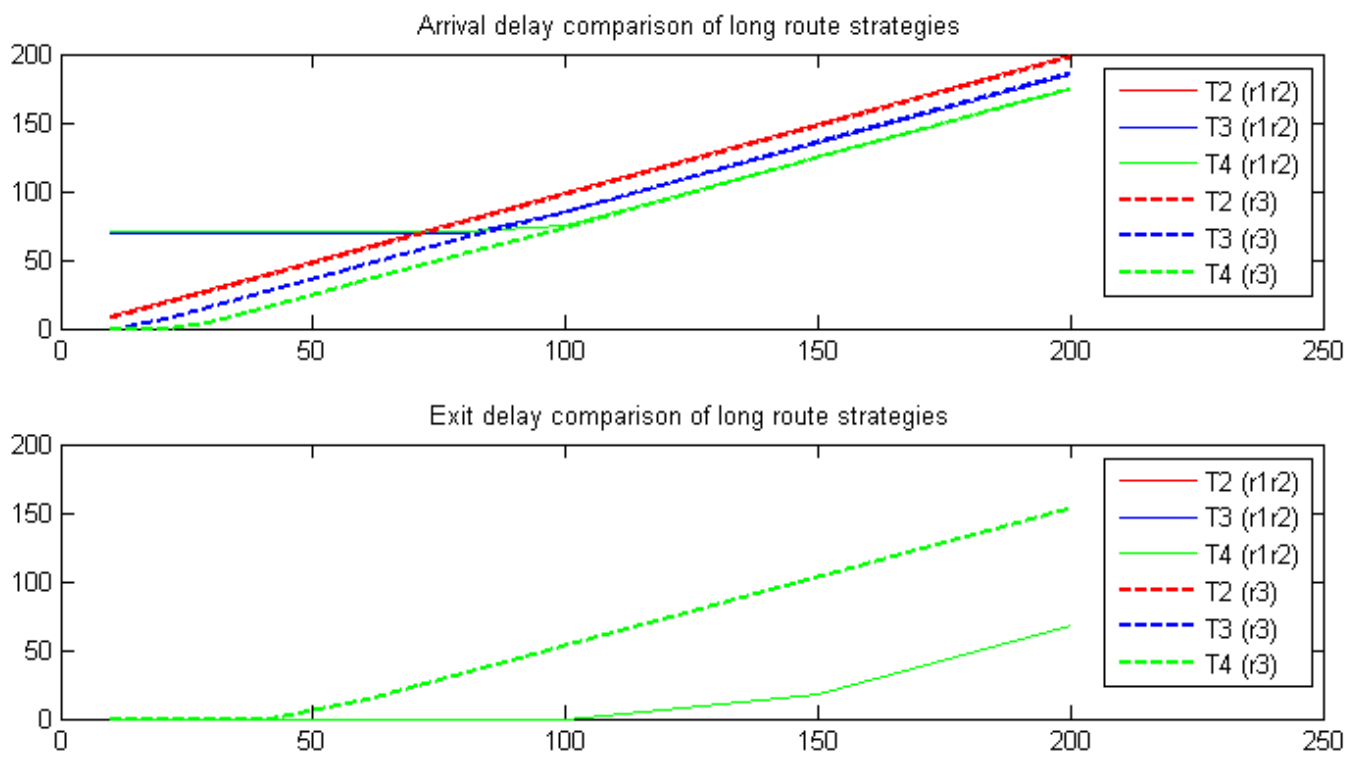

Figure 6-8 Routing solutions under different values of initial delay

It is obvious that when the initial delay is less than 40 time unit, exit delays in both strategies can be minimised to zero, thus the solution of route 3 worked out by extended skills is optimal, with less arrival delays. Instead, alternate routes 1 and 2 can be a better solution when the initial delay is greater than 80 time unit, where arrival delays are not 
significantly different by the two strategies. However, strategy with route 3 may probably enlarge the exit delays. Thus, after verification the following experience can be added to the D-Agent of station A:

- Route 3 can be assigned to minimise the total delays if the initial delay is less than 40 time unit.

- Alternate routes 1 and 2 (as planned) remain a better solution if the initial delay is greater than 80 time unit.

\subsubsection{Decision support}

If the two objective functions lead to different solutions (when the initial disturbance is between 40 and 80 ), the D-Agent has to propose a final decision.

Take Figure 6-8 as an example where the initial delay is 60 time unit, the D-Agent has estimated that there might be a conflict for train 3 and train 1 at segments 1 and 2, as well as the one for train 4 and train 2 at segment 7 . To avoid these conflicts in the subsequent traffic, theoretically the D-Agent has to provide all possible solutions. Graphically, Figure 6-9 and Figure 6-10 show the estimated conflicts and the comparison of feasible solutions, where the skill $n+3$ with objective (5-1) suggests strategy of route 3 that minimises the total arrival delay (marked by red dotted lines), however skill $n+3$ with objective (5-2) works out the inverse results since the strategy with route 3 leads larger total exit delay (marked by green solid lines).

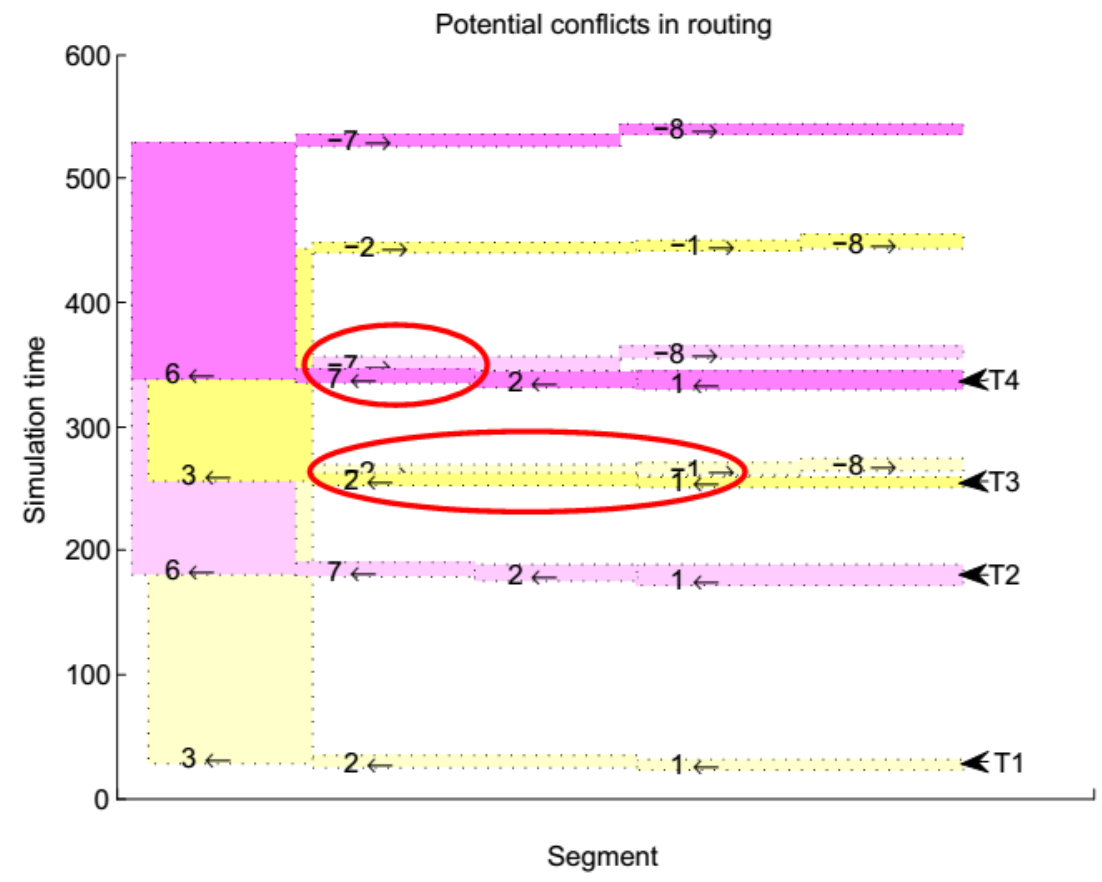

Figure 6-9 Estimated conflicts in routing when train 2 is 60 time unit delayed 


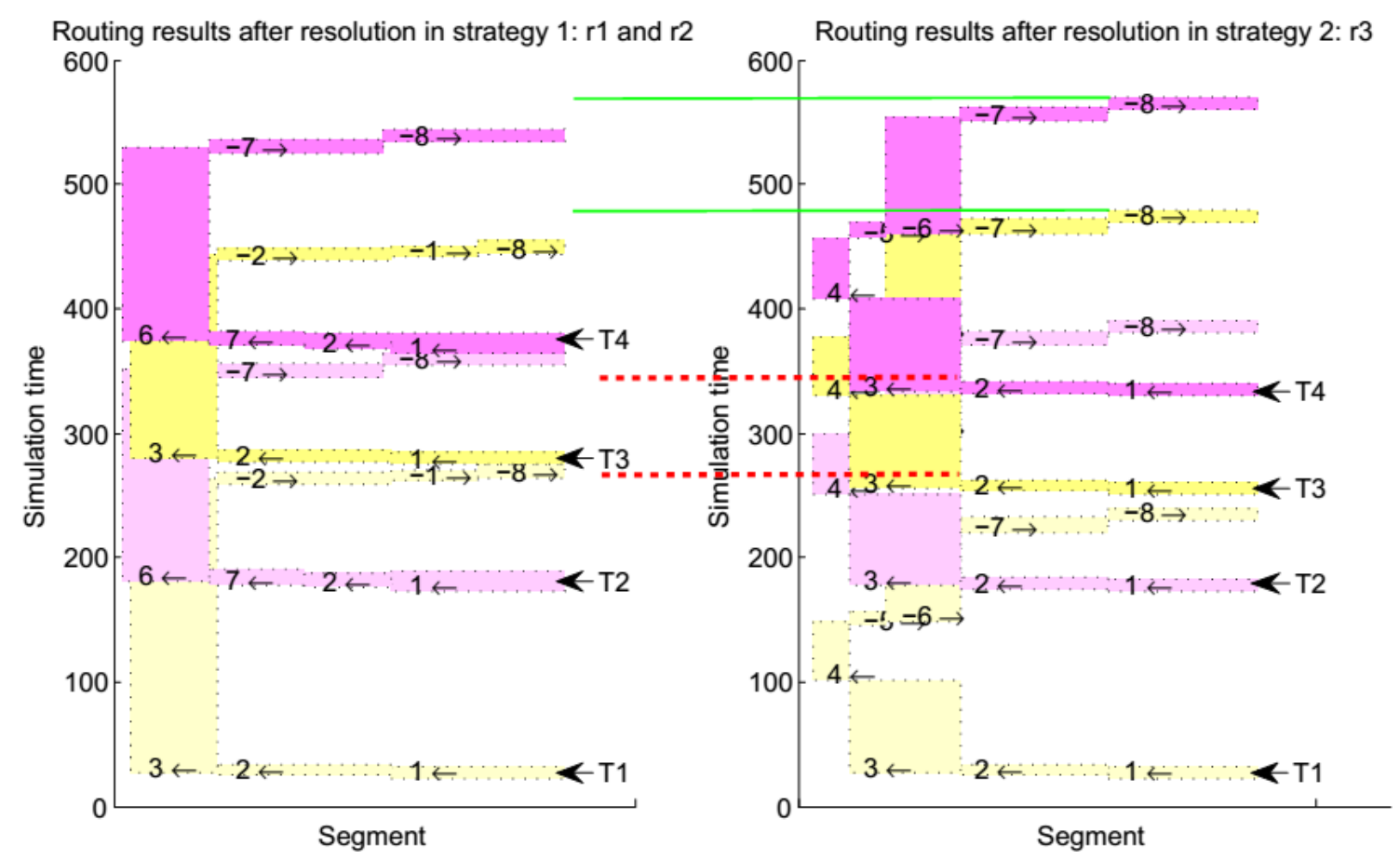

Figure 6-10 Routing results in different strategies

In this case, the D-Agent obeys the following rules to make its decision:

- If the D-Agent works in mode $\mathrm{S}$ (supporting) and the dispatcher does the operations, it accepts the order from dispatcher;

- If the D-Agent works in mode A (autonomous), it uses an objective function by linear superposition of (5-1) and (5-2) to substitute for the objective in skills $n+1$ to $n+3$.

In practice, it can be further considered the factor of total number of delayed trains or the total number of trains that have to be adjusted if the operation rules are allowed. Table 6-5 shows the estimated delays after local adjustment in different strategies. Since there is no order from dispatcher in above example, route 3 has been suggested by the D-Agent when the initial delay values 60 time unit. Nevertheless, the dispatcher may have authority to select a mandatory solution rather than accept the one proposed by the D-Agent, e.g. according to Table 6-5 a dispatcher may insist on adopting alternate routes 1 and 2 to achieve zero delayed when trains exit from station $\mathrm{A}$.

Table 6-5 Estimated optimisation results in different strategies

\begin{tabular}{l|ccc|ccc}
\hline \multirow{2}{*}{ Delay } & \multicolumn{5}{|l}{ Strategy 1: routes 1, 2 } & \multicolumn{4}{l}{ Strategy 2: routes 3 } \\
& Train 2 & Train 3 & Train 4 & Train 2 & Train 3 & Train 4 \\
Arrival & 58.6 & 69.6 & 70.6 & 57.6 & 46 & 34.4 \\
Exit & 0 & 0 & 0 & 14 & 14 & 14 \\
\hline
\end{tabular}

Table 6-6 shows part of the operational advice given by the D-Agent in chronological order that is to be translated to concrete messages to communicate with other entities in the environment of the D-Agent. 
Table 6-6 The operations proposed by D-Agent (in strategy 2: route 3)

\begin{tabular}{|c|c|c|}
\hline $\begin{array}{l}\text { Operation } \\
\text { index }\end{array}$ & \multicolumn{2}{|c|}{$\begin{array}{l}\text { Proposed operations in } 180 \text { time unit after train } 2 \text { enters station A with } 60 \\
\text { time unit delay }\end{array}$} \\
\hline 1 & 172.6 & Train 2 enters \\
\hline 2 & 203-236 & Train 2 stops at track I for 30 time unit \\
\hline 3 & 251 & Train 3 enters \\
\hline 4 & $273-283$ & $\begin{array}{l}\text { Train } 2 \text { arrives at shunting track, } 10 \text { time unit for } \\
\text { changing the direction }\end{array}$ \\
\hline 5 & $295-325$ & Train 3 stops at track I for 30 time unit \\
\hline 6 & 330 & Train 4 enters \\
\hline 7 & $330-360$ & Train 2 stops at track II and prepares to depart \\
\hline 8 & $352-362$ & Train 3 changes the direction \\
\hline
\end{tabular}

\subsection{Conclusions}

In this chapter, an agent-based CDR support system is designed, so-called D-Agent. Six basic modules: local database, knowledge base, skill base, data processing, reasoning mechanism and communication interfaces compose the D-Agent, in which the approaches of conflict detection and resolution are implanted as the kernel skills.

The modular design of the D-Agent allows the data, knowledge, reasoning logic, linguistic abilities and other skills to be integrated flexibly in an individual. Each DAgent can be distinct from others by heredity and learning. A D-Agent is not changeless even after it has been created or has come into service. It is open to new knowledge and new methods. From the simulation results we can find new possible solutions, which might have not been considered by human dispatchers or might still be discussed. However, from an evolutionary perspective, it allows various dispatching strategies and methods to be planted into the agent. By proper training and testing, the D-Agent can learn and obtain experience gradually according to different scenarios. Furthermore, with appropriate self-evaluation, it can reasonably assess the strategies and methods and support the decision-making in station operation.

The skill extension with MILP formulations is novel. However, the result reveals that it is also feasible to apply rigid mathematical formulations in a relatively flexible model of agent. The integration allows the support system not only to be rule-based, but also to solve problems with other introduced methods that have good performance in similar problems. The methods are not limited to mathematical formulations.

It is prepared for the adaption to different railway systems.

Even though there are a lot of potential advantages to apply agent-based method in railway traffic dispatching, it is not easy work to create agents as complicate and smart as we expect. There are many difficulties in getting full authority to access real-time data, to extract all knowledge of the stations and the dispatchers, to generate all kinds of 
skills, and to accurately simulate all the logic, etc. However it is promising and predicting a great deal of work to do.

- First, multi-agent modelling on the base of D-Agent is the very closely related work, since the MAS approaches may contribute to a collective intelligence that is not evident in individual D-Agent. For example, the rescheduling problem in variable scope or at global level (at network level) that has not been discussed in this chapter, may be settled by using MAS approaches, where the D-Agent can be an autonomous agent to the centralized traffic controller as well as a mediator agent to the local trains and infrastructure.

- Meanwhile, relative algorithms and methods in routing problem can be introduced as new skills of the D-Agent to improve its efficiency and adaptability.

- The training work of the D-Agent before it takes part in addressing the real problem is also necessary in order to enrich its experience and reduce the calculation time in practice.

- Last, more systematic behavioural study of different dispatchers and corresponding operational scenarios may actually helpful in the development and implement of such kind of support systems. 
Chapter 7

CDR in different ERTMS/ETCS application levels

Impacts of different ERTMS/ETCS application levels on corresponding CDR realisation are discussed. 


\section{Chapter 7 CDR in different ERTMS/ETCS application levels}

The ERTMS/ETCS system in different application levels mainly defers from the operating relationships between trackside and train.

In this chapter, we will discuss whether the application level of ERTMS system actually affects the functions of CDR and whether the awareness of system level is a necessary condition of CDR. Figure 7-1 shows the main information that is involved in conflict detection and resolution in an ERTMS/ETCS system. Only the applications in ERTMS/ETCS Level 1, 2, 3 are considered. Not all information in Figure 7-1 is obliged in each ERTMS/ETCS application level.

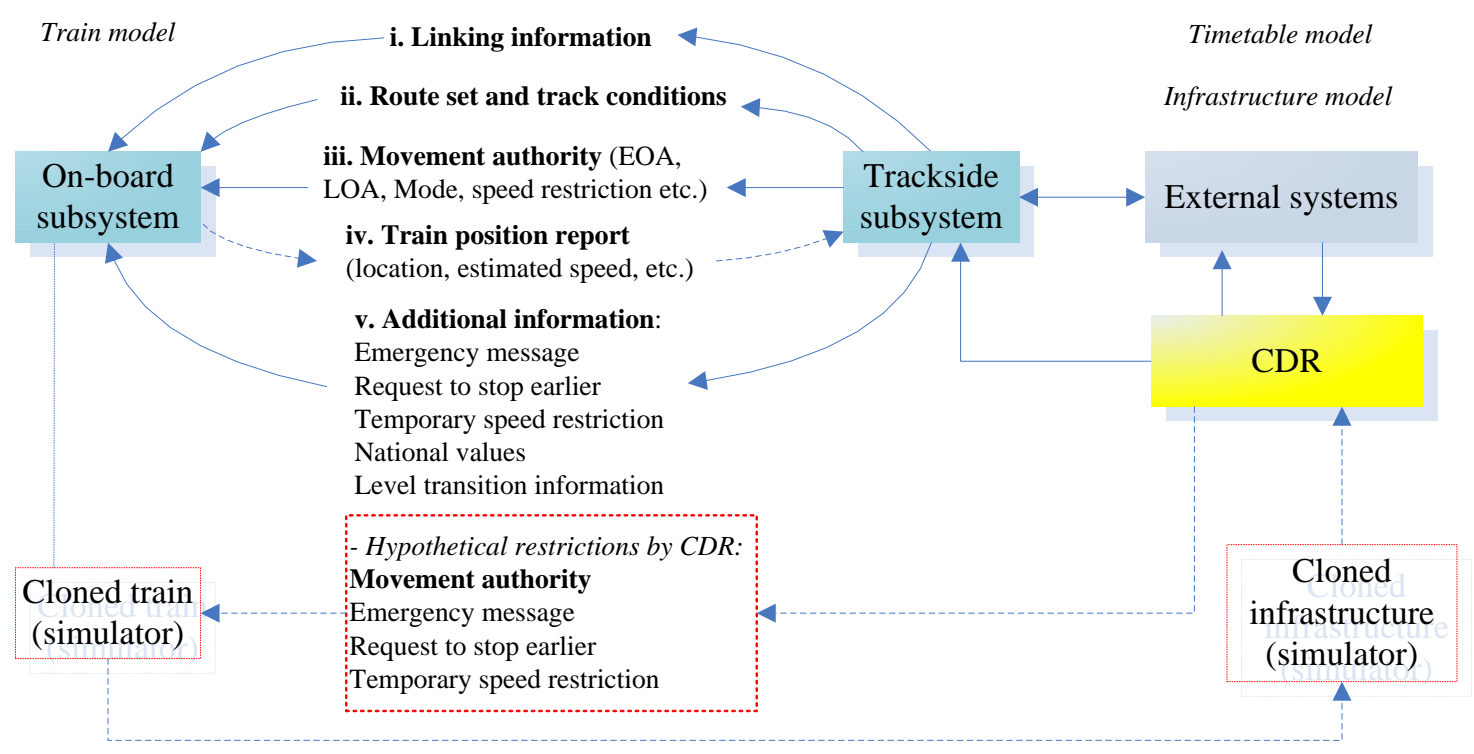

Figure 7-1 Exchanged information between CDR and ERTMS/ETCS system

To acquire the concrete contents of each category of information, some key techniques that may have significant impact on the application of each ERTMS level as well be related with the conflict detection and resolution are firstly studied. They are train detection and train positioning technique (on board positioning system), minimum safety headway and train movement authority.

\subsection{Train detection}

The train detection data is the dynamic input of the conflict detection and resolution procedure. 


\subsubsection{Technique and accuracy}

Train detection can be realised by track occupancy detection in the conventional railway network, where the trains are controlled under fixed block signalling system and the detection precision is at block level. Trains are tracked corresponding to the state changes of the blocks.

Train positioning can be considered as the category of train detection with higher accuracy in train location detection. As a fact, precise train positioning is one of the key techniques in the modern railway systems, especially in those systems with high speed trains or with trains in high frequency. The development and implement of moving block signalling system also relies on the precondition of the high reliability and accuracy of train positioning. The precision level train positioning depends on the concrete position system (the equipment and methodology). Multi-sensors are usually used to provide relative measurements and other equipment is combined to provide absolute location references, such as the combination of odometer and Eurobalises. Various integrated approaches are also developed to achieve more satisfying positioning results.

So far, the train detection and train integrity supervision are performed by the underlying signalling system (interlocking, track circuits, etc.) in ERTMS Level 1 and Level 2, and only in ERTMS Level 3 they are performed by the radio block centre (RBC) in co-operation with the train, although the train positioning system is included on the ETCS Level 2 equipped train and it also send position reports to the RBC.

In conflict detection and resolution, it is assumed that all the train detection data, independent on the system that is in charge of the detection, is reliable at its predefined accuracy level. In other words, only accuracy level of train data will be distinguished by the CDR, not the more detailed confidence intervals of the position information (such as the distance accuracy and speed accuracy required in 5.3 of SUBSET-041 (UNISIG, 2012)).

\subsubsection{Position information}

The position information (for CDR) has to be given at least in one of the following ways:

- Track occupancy data: at least including the tracked train ID, occupied block or blocks, train running direction (the direction of the block control) and the time stamp of train entering or exiting from a block.

- Train positioning data: at least including the individual train ID, the train head position (the front end of the train) in relation to the track kilometre, the direction of train movement, the estimate speed and the time stamp of data collection. 
According to the train detection data (and its responsible system), conflict detection and resolution approaches may be applied. For example, the state transition approach is the premier option in conflict detection if the train data is based on track occupancy supervision; while the train trajectory approach is preferable for its direct adaption to the precise train positioning data; also, both approaches can be combined at the same time.

Blocks can be introduced as segments in the infrastructure model presented in Chapter 3 and Chapter 4 . Train positioning data corresponds to the variables $g^{i}(x, y, t, v)$, where the track usage information $y$ is still achieved from the trackside system (it may be provided by the up-to-date positioning system with more precise geography data, but it is not within the scope of this work).

\subsection{Minimum headway distance}

Dynamic minimum headway calculation is conductive to supervise whether the train moves in its safety headway restrictions, and it also provides an important basis for taking appropriate measures in real-time traffic management.

As mentioned in previous chapters, the minimum headway distance from a current train $i_{2}$ to its foregoing train $i_{1}$ is expressed as a function $h\left(t, v_{1}, v_{2}\right)$. This function is discussed in different cases, by taking account of the target point ahead of the current train. Here a target point, referring to the definition of danger point in ERTMS/ETCS system requirements specification (ERA et al., 2014), can be (not exhaustive list):

- the entry point of an occupied block section (if the line is operated according to fixed block principles);

- the position of the safe rear end of a train (if the line is operated according to moving block principles);

- the fouling point of a switch, positioned for a route, conflicting with the current direction of movement of the train (both for fixed and moving block mode of operation).

In this work, the situation that the train has to stop or reduce speed before a joint point for the purpose of yielding to another train corresponds to the last category. As mentioned in 5.1, in this case, the additional distance to ensure the train moves without a risk for a hazardous situation at the danger point is valid for a type of defined time (such as minimum following interval $\Delta f^{i_{1} i_{2}}$ or $\Delta f^{i_{2} i_{1}}$ ). This category of target point is not discussed in this section.

\subsubsection{Headway under fixed block principles}

When the traffic is controlled through fixed block signalling system and the train is supervised by track occupancy detection, then the minimum headway function of the succeeding train $i_{2}$ at time $t$ can be expressed by 


$$
h\left(t, v_{1}, v_{2}\right)= \begin{cases}2 \cdot L b, & V Y_{m}<v_{2} \leq V_{m} \\ L b, & v_{2} \leq V Y_{m}\end{cases}
$$

where the maximum line speed $V_{m}$, the limited speed at the single yellow aspect $V Y_{m}$ and the block length and $L b$ are given. Figure 7-2 shows an example of these parameters under a four aspect coloured signalling system, where the block length and the maximum line speed are predefined to satisfy:

$$
L b \geq \frac{V_{m}{ }^{2}}{4 \cdot a_{b}}
$$

$a_{b}$ is the most conservative braking rate. And the limited speed at the single yellow aspect can be determined by $\sqrt{V_{m}{ }^{2}-2 \cdot a_{b} \cdot L b}$ or $\sqrt{2 \cdot a_{b} \cdot L b}$.

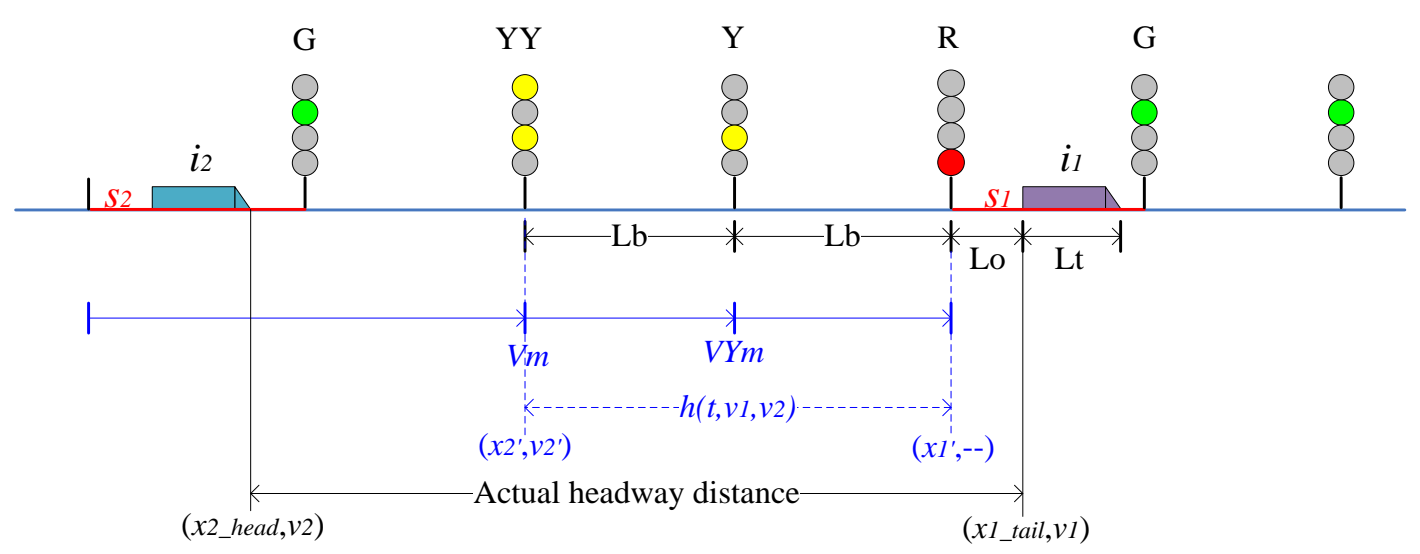

Figure 7-2 An example of headway distance under four aspect signalling system

In this case, the information required to calculate $h\left(t, v_{1}, v_{2}\right)$ is $v_{2}$, the dynamic speed of the succeeding train $i_{2}$ at current time $t$. The target point is often given as the information of the occupied block (such as the ID, the start and end locations, etc.), thus the safety headway of the succeeding train can also be described as the total number of blocks that are required to keep apart according to its current speed. Once the location information of the blocks is available, the precise positions are not obliged, neither the speed of the foregoing train. It is not limited to four aspect coloured signalling system.

\subsubsection{Headway under moving block principles}

Early in 1970s, Pearson (1973) gave out a group of functions to express the headway distance under moving block signalling control. His (concept) definitions of moving block and corresponding headway functions are partly cited in this subsection.

A moving block implies that the minimum distance separating the front end (the head) of a succeeding train $i_{2}$ from the rear end (the tail) of the foregoing train $i_{1}$ is not fixed while the trains are moving. According to the definition, the minimum headway can be 
numerically represented as the length of a moving block. Figure 7-3 shows an example of moving block.

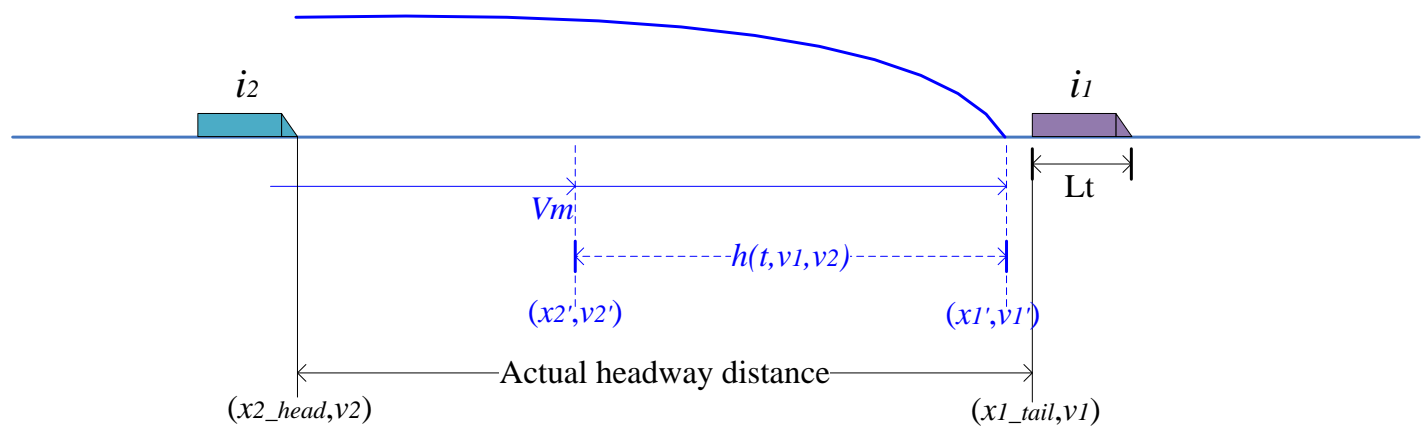

Figure 7-3 An example of headway distance under moving block operating system

\section{Moving space block}

In moving space block operation, the length of block is constant and it satisfies the minimum headway distance separating the head of a succeeding train $i_{2}$ running at the maximum line speed from the tail of the foregoing train $i_{1}$. According to the definition, the block moves with the same distance as the displacement of train $i_{2}$ varies.

$$
h\left(t, v_{1}, v_{2}\right)=\frac{V_{m}^{2}}{2 \cdot a_{b}}
$$

where $V_{m}$ is the maximum line speed and $a_{b}$ is the minimum braking rate used in deceleration stage. In this function, the minimum headway is independent on the instantaneous speed of any train (under the precondition: $v_{2} \leq V_{m}$ ). The only information required to ensure the safety headway is the precise positioning information of both trains.

Compared with the minimum headway function under fixed block signalling, the moving space block operation seems to have little superiority in reducing the absolute headway when the actual speed of train is much less than the maximum line speed most of the time. The difference may lie on the replacement of trackside signals by accurate positioning systems and the accordant communication technology.

\section{Moving time block}

In moving time block operation, the interval (headway time) between two trains passing any point along the line is constant. To represent in the distance, we calculate the moving time block length in the following expression, which is different from the original version in (Pearson, 1973).

$$
h\left(t, v_{1}, v_{2}\right)=v_{2} \cdot I_{\min }
$$

where $I_{\min }$ is the minimum line interval and it is obtainable according to the given line and train characteristics (for instance, Figure 7-4 shows how the minimum interval is 
determined by taking the maximum line speed and other key points of the line into account). It is vital that in moving time block operation, the precise speed $v_{2}$ is required.
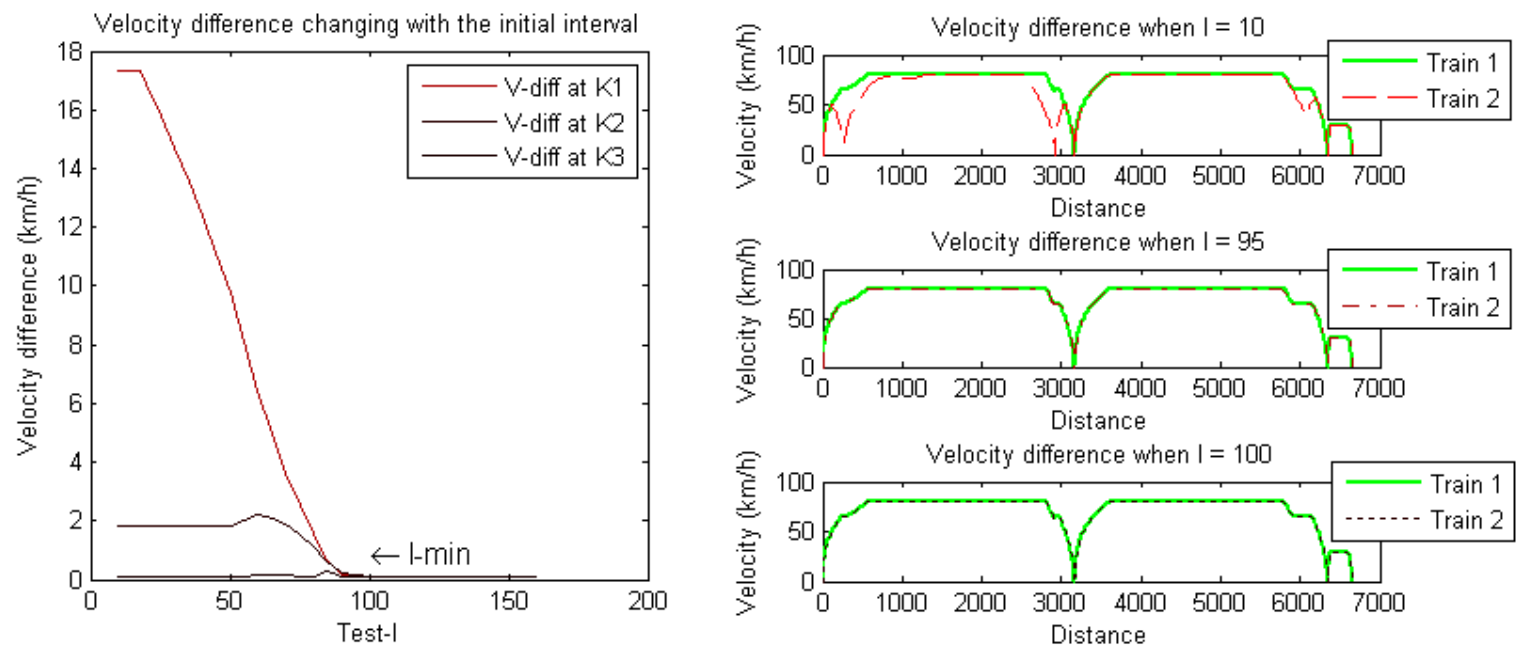

Figure 7-4 An example of minimum line interval satisfying the minimum headway time between successive trains that may run at the maximum line speed, at each key point of the line (K1, K2, K3 in the figure represent different key points).

Whether the moving time block operation can reduce the absolute headway and provide a greater line capacity compared with the moving space block operation is to be discussed, according to the specific line and equipped trains. Continuous communication between the train and trackside systems is required.

\section{Pure moving block}

Under pure moving block operation, the block length is the dynamically determined by the speed of the succeeding train $i_{2}$. The corresponding minimum headway function is given by

$$
h\left(t, v_{1}, v_{2}\right)=\frac{v_{2}^{2}}{2 \cdot a_{b}}
$$

So long as running in the same direction, the speed of the foregoing train $i_{1}$ does not affect the absolute value of the minimum headway distance. However, to keep a safety headway distance, the relative positions of the trains are required.

Since the train speed is limited as $v_{2} \leq V_{m}$, the headway distance controlled by the pure moving block system can always achieve less than or equal to that controlled by the moving space system. The condition to implement the pure moving block system is that the train speed is precisely detected and efficiently communicated.

\subsubsection{Factors of minimum headway control}

To compare the minimum headway distance calculated by (7-1) and (7-2a/b/c), we may make an example, supposing that the maximum line speed $V_{m}$ is $80 \mathrm{~m} / \mathrm{s}(288 \mathrm{~km} / \mathrm{h})$ 
and the speed profiles and the minimum interval for the specific section are given. Figure 7-5 shows these speed profiles and the corresponding values of the minimum headway under fixed block, moving space block, moving time block and pure moving block systems.
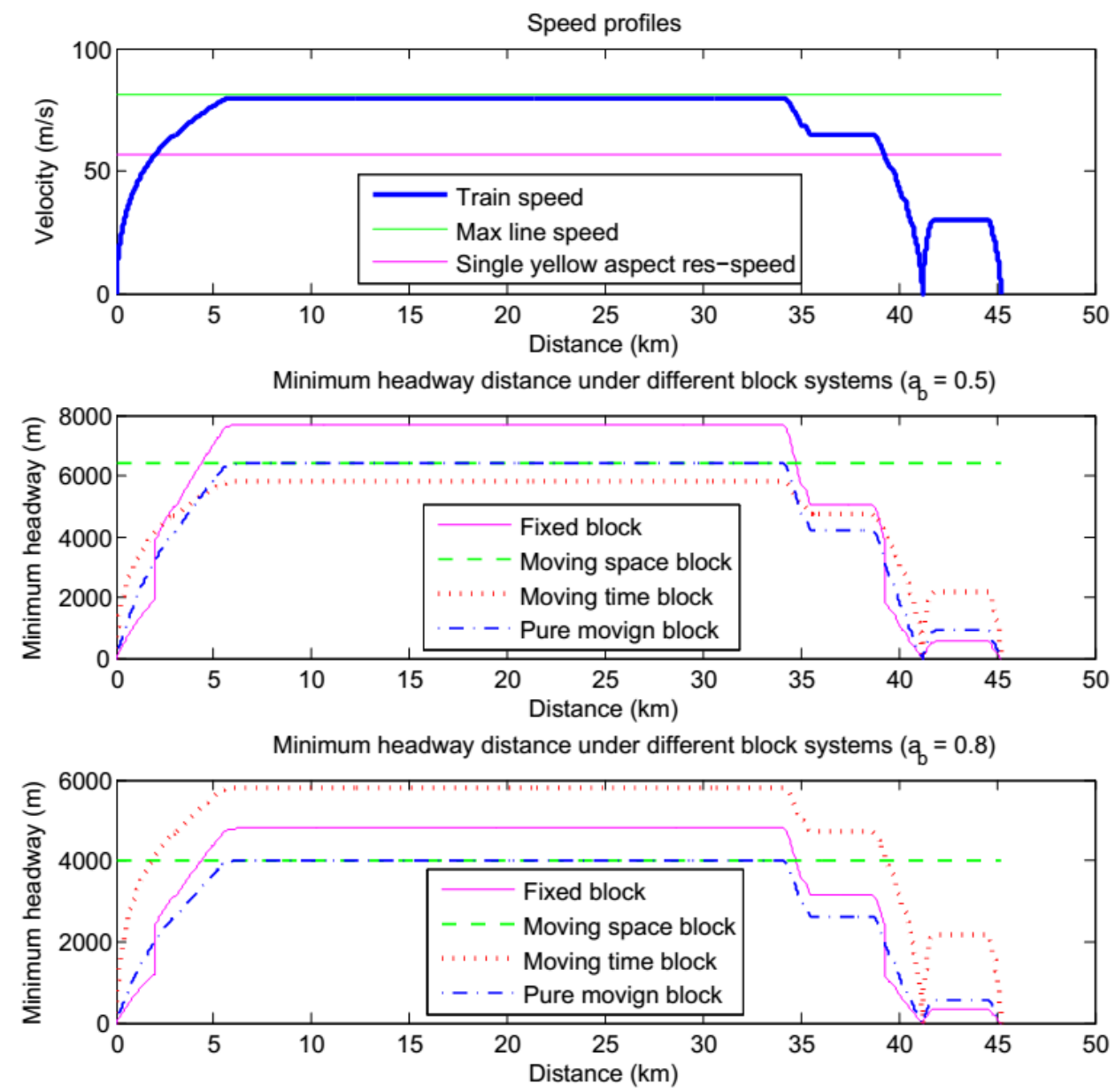

Figure 7-5 A comparison of minimum headway distance under different signalling systems and different braking characteristics (represented by the minimum braking rate $a_{b}$ )

Besides the factor of signalling system, different minimum braking rates are used to compare the performance of different signalling systems in reducing the minimum headway distance. The results imply that hardly any of the signalling system is always able to keep better than others. Concrete train speed restrictions, the characteristics of train and the minimum line interval, etc. may influence the system performances. Nevertheless, it shows that the minimum headway distance is obtainable and comparable if the line configurations, the train characteristics and the signalling system are given.

Other specific factors of the minimum headway distance still include (but not limited to) the gradients and the curves of line, the granularity of train detection, etc. 


\subsection{Train movement authority}

According to the ERTMS/ETCS system requirements specification, a movement authority (MA) is the permission for a train to move to a specific location with supervision of speed. MA, the limitations related to MA, the track description covering as a minimum the whole distance defined by the MA and the linking information realise the completeness data for safe train movement.

\subsubsection{Content of MA}

MA mainly contains the following aspects:

- The target distance: indicated by the end of authority (EOA). The distance from the current location of train to the EOA is the authorised (safety) headway of the train to move.

- The target speed at the EOA: indicated by the limit of authority (LOA). When the target speed at the EOA is not zero, LOA is given as the permitted speed of train.

- Danger point information: if any, including the distance from the EOA to the danger point and the release speed related to danger point.

- Overlap information: if any, including the distance from the EOA to the end of overlap, release speed related to overlap, etc.

A release speed is a speed limit under which the train is allowed to run in the vicinity of the EOA, when the target speed is zero. More details about the structure of the MA refer to (ERA et al., 2014).

Since the content of the MA is controllable, by the entity that has the accessibility of traffic control, MA can be one of the entrances for conflict resolution. First, if any potential conflicts are detected, they can be reported as the danger points; Second, the safety headway distance based on the minimum headway functions presented in 7.2 can be calculated to propose the EOA; Third, if the EOA cannot ensure the safety before any danger point, new speed profile is considered (in order to reduce speed or stop) and speed restrictions and satisfied EOA are updated; Last, if any overlap exists, take the additional distance or additional operation time into account.

\subsubsection{Radio block centre}

Radio block centre (RBC) is the wayside system designed to control railway traffic in ERTMS Level 2/3. It acquires signalling related information (e.g. block occupancy, route set information, etc.) from associated interlocking equipment and provides movement authorities to trains within its controlled area.

The RBC can also be directly interfaced with traditional relay-based interlockings. The RBC can even be interfaced directly with the field objects, and so it can perform 
some of the functionalities traditionally assigned to interlockings. In other words, in ERTMS Level 2 and Level 3, CDR at RBC may realise its functions by directly supporting the RBC.

Different from the MA of traditional trackside system, the MA from RBC is sent to each individual train. Emergency messages, the request to stop earlier, temporary speed restrictions (TSRs) and the inhibition of revocable TSRs can also be sent from the RBC in this way.

At the same time, the RBC may involve in the remote supervision and has interface with the centralised traffic control (CTC). CTC is the system that is not considered to depend on the application level of ERTMS/ETCS. In this way, the CDR support may also lie on the traffic management in CTCs.

\subsection{CDR in different application levels}

Table 7-1 is used to conclude the major factors that might affect the way to realise conflict detection and resolution functions in different ERTMS/ETCS application Levels.

Generally, the subsystems (or components) related with the data sources are directly concerned in conflict detection, such as the underlying signalling system, on-board position system and speed monitor, etc. Although train detection and train integrity supervision are outside the scope of ERTMS/ETCS, the data provided by these subsystems is one of the most important factors that may have direct influence on the corresponding approaches applied to detect the potential conflicts. In contrast, the subsystems (or components) related with the control commands and decisions, such as movement authorities and other additional information to the train, the signalling control to the infrastructure, etc. are referred to when conflict resolution is needed.

If the conflict detection and resolution support system does not work independently on the CTCs or RBCs (or other traffic management systems), the specific communication technology applied in different ERTMS levels is not directly associated. However, they can be the optional interfaces taken into consideration when the movement authorities are generated. Moreover, a level adapter is proposed for the sake of system interoperability between different application levels. 
Table 7-1 Factors in different ERTMS/ETCS application levels

\begin{tabular}{|c|c|c|c|c|}
\hline $\begin{array}{l}\text { ERTMS/ETCS } \\
\text { Application level } \\
\end{array}$ & Level 1 & Level 2 & Level 3 & $\begin{array}{l}\text { Direct influence } \\
\text { on CDR? }\end{array}$ \\
\hline $\begin{array}{l}\text { Train detection } \\
\text { and integrity } \\
\text { supervision }\end{array}$ & $\begin{array}{l}\text { Underlying } \\
\text { signalling } \\
\text { system }\end{array}$ & $\begin{array}{l}\text { Underlying } \\
\text { signalling } \\
\text { system }\end{array}$ & $\begin{array}{l}\text { On-board, and } \\
\text { trackside RBC } \\
\text { in co-operation } \\
\text { with the train }\end{array}$ & Yes, CD \\
\hline $\begin{array}{l}\text { Speed } \\
\text { supervision }\end{array}$ & $\begin{array}{l}\text { On-board } \\
\text { continuous }\end{array}$ & $\begin{array}{l}\text { On-board } \\
\text { continuous }\end{array}$ & $\begin{array}{l}\text { On-board } \\
\text { continuous }\end{array}$ & Yes, CD \\
\hline $\begin{array}{l}\text { Dynamic speed } \\
\text { profile } \\
\text { calculation }\end{array}$ & On-board & On-board & On-board & Yes, CR \\
\hline Train positioning & -- & $\begin{array}{l}\text { Odometer, } \\
\text { Eurobalises and } \\
\text { other media }\end{array}$ & $\begin{array}{l}\text { Odometer, } \\
\text { Eurobalises, and } \\
\text { other media }\end{array}$ & Yes, CD \\
\hline $\begin{array}{l}\text { Position } \\
\text { information } \\
\text { transmitter }\end{array}$ & -- & Euroradio & Euroradio & Yes, CD \\
\hline MA generator & Trackside & Trackside & Trackside & Yes, CR \\
\hline MA transmitter & Eurobalises & Euroradio & Euroradio & Yes, CR \\
\hline Signals & $\begin{array}{l}\text { Required, except } \\
\text { if semi- } \\
\text { continuous infill } \\
\text { is provided. }\end{array}$ & Optional & No & Yes, CR \\
\hline Eurobalises & Yes & $\begin{array}{l}\text { Yes, for location } \\
\text { referencing }\end{array}$ & $\begin{array}{l}\text { Yes, for location } \\
\text { referencing }\end{array}$ & \\
\hline $\begin{array}{l}\text { Euroloop or } \\
\text { radio infill }\end{array}$ & $\begin{array}{l}\text { Optional, semi- } \\
\text { continuous infill }\end{array}$ & No & No & Yes, interfaces \\
\hline Euroradio & No & $\begin{array}{l}\text { Yes, bi- } \\
\text { directional }\end{array}$ & $\begin{array}{l}\text { Yes, bi- } \\
\text { directional }\end{array}$ & \\
\hline $\mathbf{R B C}$ & No & $\begin{array}{l}\text { Yes, to each } \\
\text { train }\end{array}$ & $\begin{array}{l}\text { Yes, to each } \\
\text { train }\end{array}$ & Yes, CDR \\
\hline Level transitions & To Level 2, 3 & $\begin{array}{l}\text { To 2: RBC } \\
\text { hand-over } \\
\text { To Level } 1,3\end{array}$ & $\begin{array}{l}\text { To 3: RBC } \\
\text { hand-over } \\
\text { To Level 1, } 2\end{array}$ & $\begin{array}{l}\text { Yes, level } \\
\text { adapter }\end{array}$ \\
\hline
\end{tabular}

\subsubsection{In application Level 1}

In ERTMS/ETCS Level 1, the train data collection is basically performed by the trackside Interlocking and track circuits. The transmission devices can be Eurobalises or other semi-continuous infill (Euroloop or radio infill), but the continuous train speed supervised on-board is not accessible by the trackside. As Figure 7-6 shows that only the track occupancy data can be first obtained and represented by the infrastructure model. Consequently, the state transition approach is firstly applied to the conflict detection. The train trajectory conflict detection approach is an optional supplementary only when the train model is achievable according to the position information of the route, block, or switches, etc. Another difficulty of conflict detection in Level 1 is the trains may not be distinguished by their ID from the infrastructure state data. 


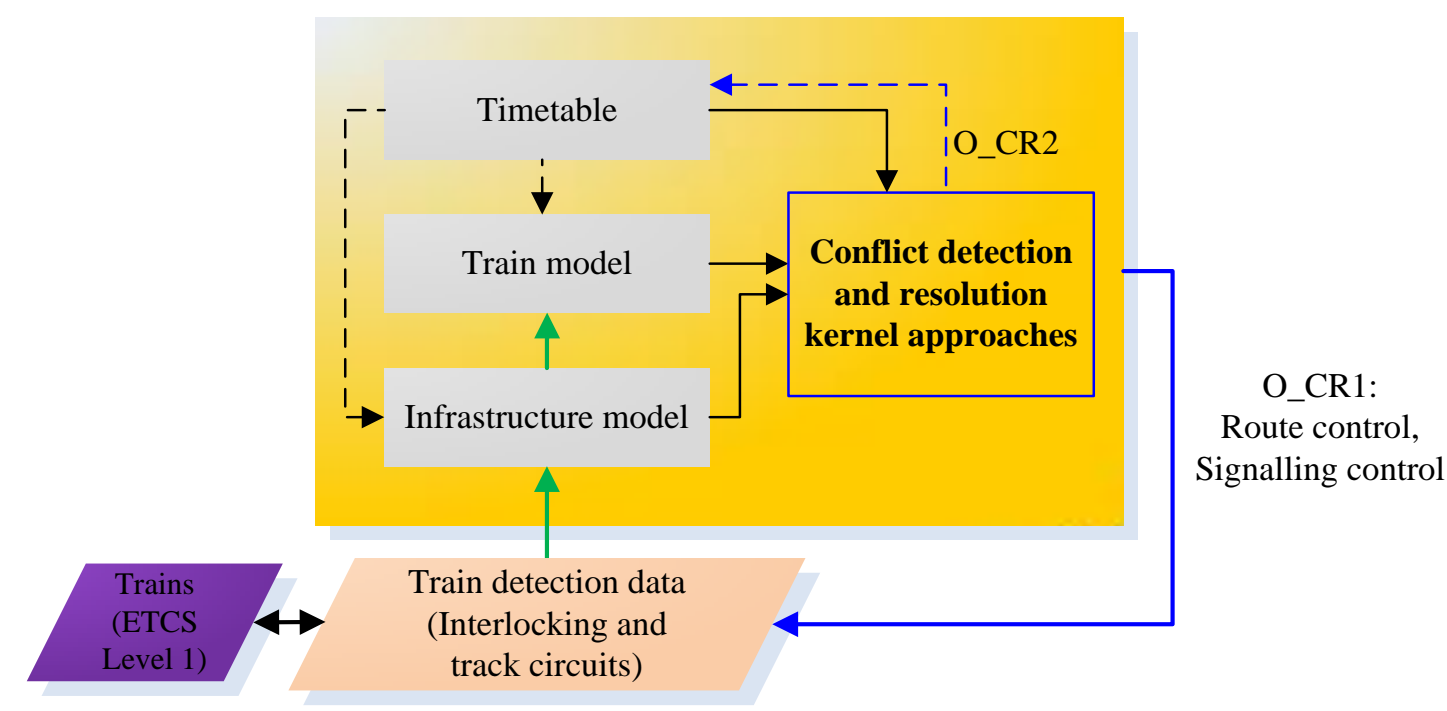

Figure 7-6 CDR in ERTMS/ETCS Level 1

If any potential conflict is detected, it is reported in the form of a danger point, where especially, the danger point is an (estimated) occupied block or a conflicting route.

Thus the local routing approach (whose output is denoted by O_CR1 in Figure 7-6) and the rescheduling approach (if necessary, whose output is denoted by O_CR2) are employed to find the solution. Minimum headway calculation and the speed restriction corresponding to the current state of signalling are prepared to generate the movement authority. Additional information (e.g. temporary speed restrictions or level transition order) is possible to be combined when needed. In CDR support system, the integrated solution is tested by predictive simulation (as shown in Figure 7-1). The approved solution, which in Level 1 is the adjusted signalling control and available route description, is sent to the underlying system and trains. Although the MA generator and transmitter do not know the train to which they send the information. The train (or the driver) can adjust the train movement according to the signals (either lineside or onboard) under the speed permitted by the MA.

\subsubsection{In application Level 2/3}

ERTMS/ETCS Level 2 and Level 3 are radio based train control systems. Besides the trackside infrastructure state data, the train positioning data (with estimated speed) is also continuously available for trackside traffic management system, particularly the RBC. Then parallel channels of data (shown in Figure 7-7) are formed as the input of the CDR support system. Both train trajectory approach and state transition approach can be applied to the conflict prediction, independently or in an integrated manner. Position information with higher accuracy may increase the certainty in predicting the transition time of state as well. 


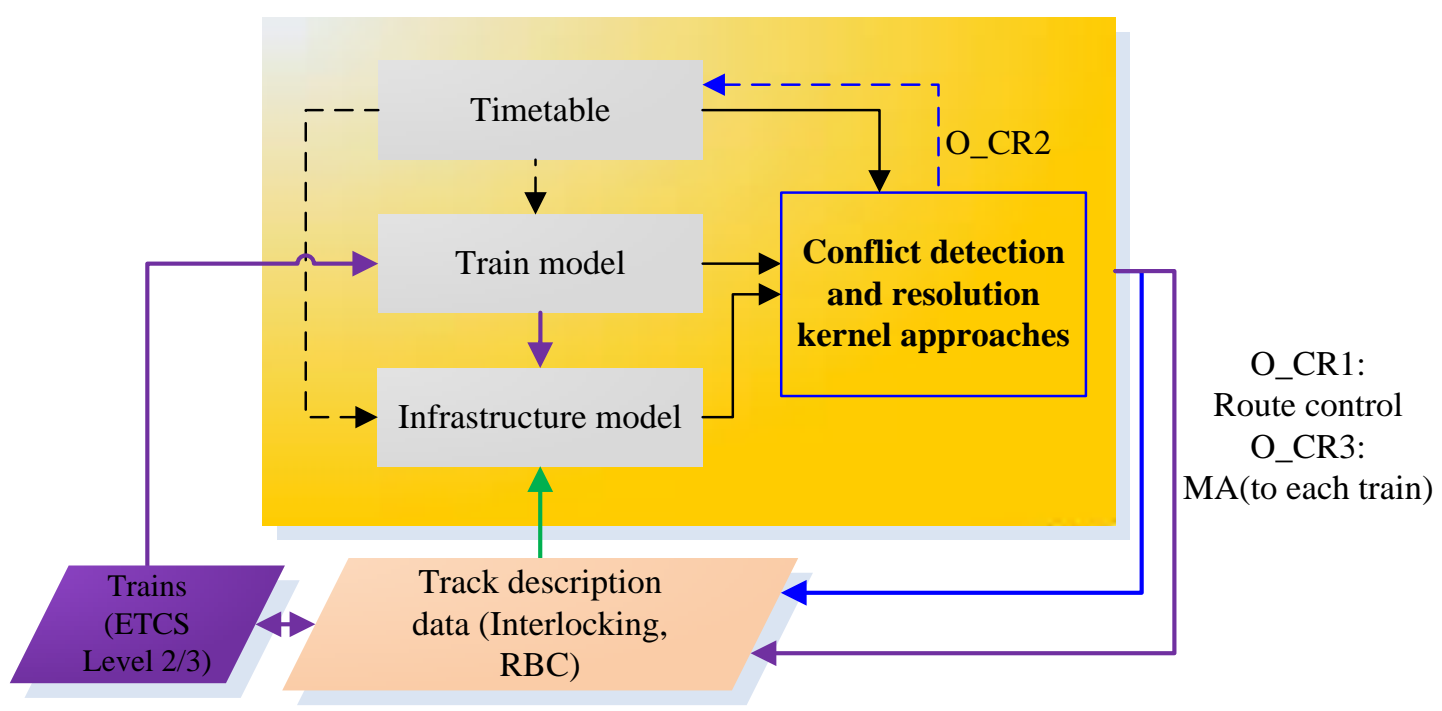

Figure 7-7 CDR in ERTMS/ETCS Level 2/3

In ERTMS/ETCS Level 2 and Level 3, potential conflicts are also reported as danger points. A switch danger point (joint point) inside the station area is resolved as that in Level 1, by routing and signalling control (O_CR1). However, according to the signalling system (fixed block or moving block) a headway danger point can be an (estimated) occupied block or the rear end of the foregoing train.

Correspondingly, the minimum headway calculation and the speed restriction also depend on the blocking technique, as presented in 7.2. If under fixed block signalling, the block control codes are generated with corresponding speed limitations; the minimum headway function (7-1) is substituted by (7-3), where $L o$ is the overlap length.

$$
h\left(t, v_{1}, v_{2}\right)= \begin{cases}2 \cdot L b+L o, & V Y_{m}<v_{2} \leq V_{m} \\ L b+L o, & v_{2} \leq V Y_{m}\end{cases}
$$

Since in Level 2 and Level 3, each controlled train is individually known by the trackside RBC, the movement authority and track description with more unique conflict resolution proposal (if necessary) is possible to be sent to each train, whatever the signalling system is. Each train may adjust its dynamic speed profile to the received movement authority taking into account its individual braking characteristics and track description data. The communication technology used in Level 2 and Level 3 allows the higher accuracy of train control.

In Level 2 and Level 3, conflict resolution measures can also take advantage of the possibility of sending additional information to each train, such as emergency messages, request to stop earlier (before some danger point), revocations of other previous messages, etc. 


\subsection{ERTMS/ETCS level adapter in CDR support system}

Basically, it seems that the application of higher level may offer quite considerable advantages in terms of capacity increase and cost reduction. For example, the author in (Koning, 2002) investigated the difference in performance between ERTMS Level 2 with fixed block signalling system and ERTMS Level 3 with moving block, and he believed that Level 2 still maintains a safe braking distance factor that may enable higher operational speeds and reduced headways; however, in station areas, headways (intervals) in ERTMS Level 2 are smaller than in ERTMS Level 3.

Although the performance of different ERTMS/ETCS application levels may be distinct from various aspects, what indeed have impact on the CDR functions are the available data (content and accuracy), the signalling system (fixed block and moving block), the controlling size of train (group or individual), as well the concerning area (in station area or open track).

To handle these factors that may be distributed in different ERTMS/ETCS application levels, a level adapter function can be added to the agent-based CDR support system presented in Chapter 6

First, the fixed requirements and line configurations of all ERTMS/ETCS levels have to be pre-stored in the database; the level transition permission and corresponding rules are loaded as knowledge.

Then in the data processing module of D-Agent, a level inquiry combined with the predefined configurations (signalling system, train equipment, communication technologies, etc.) may fast distribute corresponding models to represent the problem, which may affect the selection of the CD and CR approaches in other modules.

The level adapter function still has to take into account the environment variation when ERTMS/ETCS level transition happens. So the direct interfaces in each application level have to be predefined as well. 


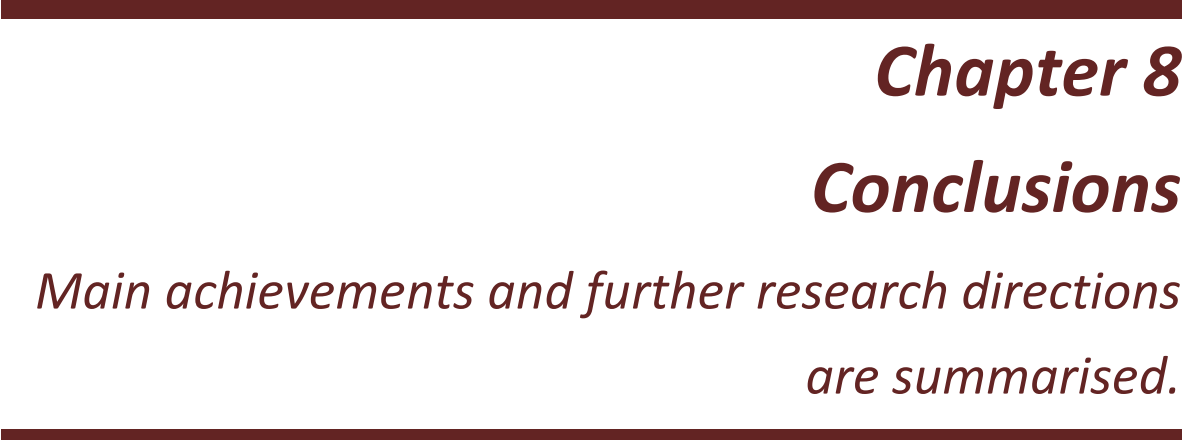




\section{Chapter 8 Conclusions}

Conflicts may exist everywhere. The work presented in this thesis aims to provide a systematic methodology of railway conflict detection and resolution at the level of traffic management and to develop an applicable conflict detection and resolution support system for railway traffic control and management system (e.g. the European Traffic Management Layer of ERTMS system).

In this chapter, the main achievements are first summarised and then some research directions for future work are suggested.

\subsection{Resume of the main achievements}

\subsubsection{Conflict sign analysis}

Since the blocking time theory in traffic prediction has obvious advantages in the accurate definition actually existing in practice and the visible abnormity in a timespace graph, it has been widely used in conflict detection and resolution. It has become the principal method to detect railway traffic conflicts in a lot of conflict related literature. However, we may still have questions, such as, "When shall we start to predict potential conflicts", "Is there any omens of conflicts", etc. Thus, in this thesis, the conflict features and possible conflict signs are firstly discussed.

In a boarder sense, we define a conflict as a situation in which the time that a train claims to use a specific part of the track overlaps with that claimed by another operation. Here the subject of an operation can be a train or other users. Thus, both the potential conflicts between trains and the ones happening to the train and the infrastructure reserved by other users are contained. On this basis, the potential conflicts are mainly classified into headway conflicts and route conflicts. The relationships between the conflicts and their signs over time are concluded in Figure 8-1, where the relationship between the two categories of conflicts is also indicated. The illustration implies that certain signs may have been incubating before conflicts arise. These signs include delays that we usually consider, as well as other deviations that might not be taken into account.

To analyse probable signs, the data-based partial models of train and infrastructure are established to describe the railway traffic, and the timetable is also multidimensionally extended. Train movement model takes into account both static speed restrictions and dynamic train characteristics. Variable train speeds are particularly considered. Infrastructure model basically contains the structural definitions (line data) and corresponding constraints. Meanwhile, it dynamically indicates the traffic state and the infrastructure availability by track description over time. It also indirectly reflects 
the general position information of train movements. Besides the conventional twodimensional time-space diagram, the extension from a regular timetable additionally involves the recommended track and speed profile for a train to follow the time-space diagram. Suspect signs based on each partial model are proposed for further calling proper conflict detection and resolution approaches.

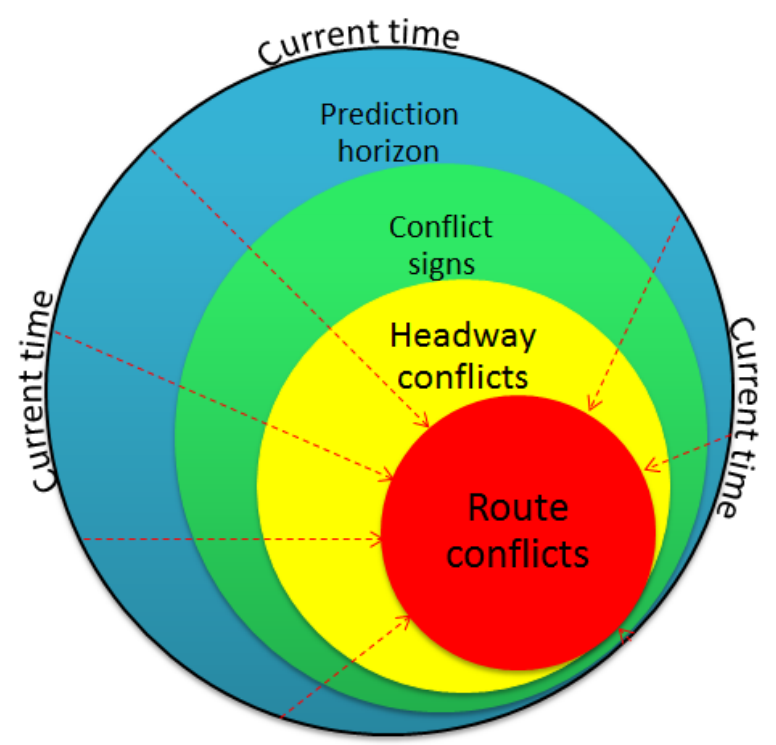

Figure 8-1 Time relationship among conflict signs, headway conflicts and route conflicts

\subsubsection{Conflict detection and resolution approaches}

If conflict signs in time, distance, train speed, and infrastructure state are detected from the collected data, conflict detection function is first activated.

This thesis makes a special effort to establish the methodology of conflict detection in Chapter 4 , where the train trajectory tracing approach and the infrastructure state transition approach are presented. Being connected, these approaches are inclined to correspond to all the partial models and therefore at least some approach can be applicable for a particular system. The train trajectory approach is accomplished in handling the detailed data of train movement. When train positions, train speeds and the operation times are available or when conflict signs in these dimensions are proposed, the correlations indicated in time-space, time-speed, and speed-space dimensions can be cooperatively checked. Headway conflicts are mainly predicted in this approach. The infrastructure state transition approach is preferable when the track occupancy data and infrastructure configurations (including temporary limitations) are directly obtainable or when precise train positioning information is lacking. Via this approach, potential conflicts caused by insufficient headway intervals and incompatible routes are commonly detected. Numeral criteria are used to estimate a conflict and its corresponding type. 
Subsequently, conflict resolution methodology is composed, oriented to various types of conflicts. Mixed integer linear programming formulations are produced to transcribe concrete conflict problems and to find feasible solutions in the described situations. In minimum interval and route conflict problems, particularly in the station area, local routing approach taking different dispatching strategies into account is suggested, where the operation times, train route selection and train orders at joint points are the adjustable variables. To prevent potential conflict propagation in a wider area, variable scope rescheduling approach is proposed, where the rescheduling scope is dependent on the concrete conflict size. The two layers are integrated to generate adaptive solutions in different conflicting situations. It is emphasised that, not only the routing and rescheduling approaches proposed in this thesis are suggested, but also any other reasonable dispatching strategies in local routing and well-performing algorithms in rescheduling are flexible to introduce to the conflict resolution methodology.

\subsubsection{CDR support system}

Separated approaches of conflict detection and resolution are consolidated to provide systemic assistance to traffic controllers or automated traffic control systems. There are two keys of a compatible CDR support system for railway traffic management:

- the adaptability to any format of the train detection data (either from the train equipment or from the trackside system) in all kinds of railway system;

- and the adjustment of the CD and CR approaches to obtain suitable solutions for the specific railway system (such as any particular application level of ERTMS).

To achieve these key functions, the D-Agent, an agent-based CDR support system is modularly designed. Local database, knowledge base, skill base, data processing, reasoning mechanism and communication interfaces of the D-Agent, respectively, realise the implantation of the line and train data, related restrictions and control rules, proposed data-based models and CDR approaches, and the practical communication protocols. Particularly, the communication interfaces allow the D-Agent to keep updating the collective data from the environment and timely deliver feedback information to upper decision makers. The data-based models and matched CD approaches connect the traffic environment and the traffic control. Conflict identification and classification prepare for the application of CR approaches and other excellent methods and algorithms that are proposed for railway traffic optimisation. Then, the reasoning mechanism of the D-Agent aims to simulate the basic intelligent characteristics in the traffic control and management, such as the behaviour logic of a dispatcher. A D-Agent is prepared for the adaption to different railway systems. 


\subsubsection{CDR in ERTMS system}

To apply the CDR support system in an ERTMS system, characteristics of different ERTMS/ETCS application levels are firstly investigated. Some key techniques, including train detection and train positioning technique (on board positioning system), minimum safety headway and train movement authority, are taken into account.

Different ERTMS/ETCS applications have distinct performance. Correspondingly, potential conflicts, involved components and control measures in ERTMS/ETCS Level 1 and Level 2/3 are compared. Primary factors associated to the CDR functions in a particular ERTMS system lie in the available data, the signalling system and block principles, the train communication level (ETCS level) and the conflicting area.

These factors can be variable with the train travelling over regions. Besides, equipment upgrading or temporary configurations for downgrading use are also concerned in ERTMS applications. To inform these factors and possible variations to the CDR functions, a level adapter is proposed to add to the agent-based CDR support system. The adapter deals with level inquiries and the corresponding configurations at the data processing stage, and encapsulates valid control messages to adapt to the application level via communication interfaces.

\subsection{Outlook for future research}

The proposed methodologies in this thesis are simulated and analysed, not actually implemented or tested in a real operational railway system. In an application point of view, any approaches without applying to the real system are not qualified whatever advantages they have theoretically. For this reason, there are a number of issues remaining in further research.

\section{CDR support system development}

As proposed in Figure 1-2, the conflict detection and resolution support system has to be implemented parallel to a traffic management system or as a part of it.

First, the communication functions, above of all, the communication interfaces between the real system and the CDR support system should be developed and tested. It may depend on the public protocols or specific protocols generated by negotiation. In ERTMS, most interfaces are standardised. The level adapter function proposed in 7.5 should also integrate these communication interfaces.

Based on the access of practical systems, the kernel functions of conflict detection and resolution can be further extended. The conflict detection approaches proposed in this thesis have to be more formalised and systematic. Meanwhile, the blocking time theory can be imported. Performance comparison can be carried out under the same 
conditions, and complementary advantages can be taken for conflict detection. Kinds of conflict resolution approaches, which have been widely studied as rescheduling approaches and algorithms, should also be added and managed.

The intelligence of conflict detection and resolution support system is further considered. The agent-based CDR support system has provided some basic intelligence characteristics, such as judgement logic and learning. The intelligence level in fact increases with the improvement of the management of CDR skills. At the same time, other intelligence methods can also be integrated.

Furthermore, informative GUI has to be developed to support human traffic controllers or dispatchers.

\section{CDR evaluation in system simulation and testing}

The evaluation of the effectiveness and efficiency of all the model and approaches can be carried out by

- Accessing the outcome results of the objective functions, if the results are obviously satisfied (directly);

- Designing testing experiments and make contrastive analysis, such as the comparison with previous scenarios, the comparison with other algorithms and methods, etc.;

- Testing in well proved simulation and test benches.

In research of this thesis, the first two have been mainly considered. Thus, testing the CDR functions in a virtual system environment is one of the most important research directions in future work. Although the CDR support system should be implemented in a real railway system, not all tests can be carried out in the real railway system, mainly for safety and cost reasons.

To achieve this, not only the CDR support system has to be functionally designed, other subsystems at a railway system level have to been realistically simulated, where the traffic management system is a component of the entire virtual environment. Existing simulation and testing benches can be utilised in this situation, such as the ERTMS system simulation and test benches (Mera et al., 2008; Mera et al., 2002) and the CBTC (Mera et al., 2010), which have been well developed.

Test cases will be generated based on the specific railway system that is used as the virtual environment of testing and the proposed CDR support system. Meanwhile, system testing methods will be further studied.

\section{Statistical analysis of conflict data}

No matter in simulation and test environment or in a real railway system, the historical data that we obtain from the testing process are valuable for further use. 
Statistical methods can be applied to the conflict data analysis. Critical factors may be further discussed in the terms of statistics.

In this age of big data, investigating into large amounts of data in daily railway traffic management and establishing systematic big data analysis methodologies for railway systems can have both academic significance and application values.

\subsection{Publications}

Articles for international Journals:

- T. Zhu, J.M. Mera, B. Suarez, and J. Maroto. "An agent-based support system for railway station dispatching, ” Expert Systems With Applications 61 (2016) 39-52.

5-Year Journal Impact Factor: 2.571 Q1

- T. Zhu and J.M. Mera. "Railway traffic conflict detection via a state transition prediction approach, " IEEE Transactions on Intelligent Transportation Systems, DOI: 10.1109/TITS.2016.2603441.

5-Year Journal Impact Factor: 3.039 Q1

Articles for international conferences:

- T. Zhu, J.M. Mera, E. Castellote, J. López. Feasibility evaluation and critical factor analysis for subway scheduling, Computers in Railways XV, 2016.

- T. Zhu, J.M. Mera. A 3D simulation approach for railway conflict detection in Traffic Management System layer, Computers in Railways XIV, 2014. 



\section{Bibliography}

Adenso-Díaz, B., Oliva González, M., Gonzaález-Torre, P., 1999. On-line timetable rescheduling in regional train services. Transportation Research Part B: Methodological 33 387-398.

Anthony, R.N., 1965. Planning and Control Systems: a framework for analysis Harvard, Boston.

Araya, S., Fukumori, K., 1984. ESTRAC-II: An Expert System for Train Traffic Control in Disturbed Situations, Proceedings of ECAI. 1984, pp. 23-32.

Assad, A., 1980. Models for rail transportation. Transportation Research Part A 14, 205-220.

Balbo, F., Pinson, S., 2005. Dynamic modeling of a disturbance in a multi-agent system for traffic regulation. Decision Support Systems 41, 131-146.

Balbo, F., Pinson, S., 2010. Using intelligent agents for Transportation Regulation Support System design. Transportation Research Part C: Emerging Technologies 18, 140-156.

Bonabeau, E., 2002. Agent-based modeling: Methods and techniques for simulating human systems. PNAS 99, 7280-7287.

Borndörfer, R., Schlechte, T., Swarat, E., 2011. Railway Track Allocation - Simulation, Aggregation, and Optimization. Konrad-Zuse-Zentrum für Informationstechnik Berlin, Berlin-Dahlem, Germany.

Bussieck, M.R., Winter, T., Zimmerman, U.T., 1997. Discrete optimization in public rail trainsport. Math Program 79, 415-444.

Cacchiani, V., Huisman, D., Kidd, M., Kroon, L., Toth, P., Veelenturf, L., Wagenaar, J., 2014. An overview of recovery models and algorithms for real-time railway rescheduling. Transportation Research Part B: Methodological 63, 15-37.

Caimi, G., Burkolter, D., Herrmann, T., Chudak, F., Laumanns, M., 2009. Design of a Railway Scheduling Model for Dense Services. Networks and Spatial Economics 9, 25-46.

Caimi, G., Fuchsberger, M., Laumanns, M., Schüpbach, K., 2011. A multi-level framework for generating train schedules in highly utilised networks. Public Transport 3, 3-24.

Caimi, G.C., 2009. Algorithmic decision support for train scheduling in a large and highly utilised railway network, Dipl.Math.. ETH, Zurich. 
Carey, M., Crawford, I., 2007. Scheduling trains on a network of busy complex stations. Transportation Research Part B: Methodological 41, 159-178.

Carlier, J., Pinson, E., 1989. An Algorithm for Solving the Job-Shop Problem Management Science 35, 164-176.

Carlier, J., Pinson, E., 1994. Adjustment of heads and tails for the job-shop problem. European Journal of Operational Research - EJOR 78, 146-161.

Cheng, Y.-H., Yang, L.-A., 2009. A Fuzzy Petri Nets approach for railway traffic control in case of abnormality: Evidence from Taiwan railway system. Expert Systems with Applications 36, 8040-8048.

Chiang, T., Chiang, H., Ko, S., Hsieh, C., 1998. Knowledge-based system for railway scheduling, Data \& Knowledge Engineering 27, 289-312

Cordeau, J.F., Toth, P., Vigo, D., 1998. A survey of optimization models for train routing and scheduling. Transportation Science 32, 380-404.

Corman, F., 2010. Real-time Railway Traffic Management: dispatching in complex, large and busy railway networks, Department of Transport and Planning. Delft University of Technology, Netherlands.

Corman, F., D'Ariano, A., 2008. Existing and future approaches on railway traffic control from an agent-based perspective Proceedings of 10th TRAIL Congress. DUP Science, Rotterdam, The Netherlands, pp. 1-15.

Corman, F., D’Ariano, A., Hansen, I.A., Pacciarelli, D., 2011. Optimal multi-class rescheduling of railway traffic. Journal of Rail Transport Planning \& Management 1, $14-24$.

Corman, F., D'Ariano, A., Pacciarelli, D., Pranzo, M., 2009. Evaluation of green wave policy in real-time railway traffic management. Transportation Research Part C: Emerging Technologies 17, 607-616.

Corman, F., D’Ariano, A., Pacciarelli, D., Pranzo, M., 2010. A tabu search algorithm for rerouting trains during rail operations. Transportation Research Part B: Methodological 44, 175-192.

Corman, F., D’Ariano, A., Pacciarelli, D., Pranzo, M., 2012a. Bi-objective conflict detection and resolution in railway traffic management. Transportation Research Part C: Emerging Technologies 20, 79-94.

Corman, F., D’Ariano, A., Pacciarelli, D., Pranzo, M., 2012b. Optimal inter-area coordination of train rescheduling decisions. Transportation Research Part E: Logistics and Transportation Review 48, 71-88. 
Cui, Y., 2010. Simulation-Based Hybrid Model for a Partially-Automatic Dispatching of Railway Operation.

D'Addio, G.F., Mazzucchelli, M., Savio, S., 1998. Automatic conflict detection and resolution in metrorail systems: evaluation approach for MARCO EU project, Urban Transport and the Environment for the 21st Century. WIT Press, pp. 461-469.

D'Ariano, A., Albrecht, T., 2006. Running time re-optimization during real-time timetable perturbations, in: Allen, J., RUMSEY, A.F., Brebbia, C.A., Sciutto, G., Sone, S. (Eds.), Computers in Railways X, pp. 531-540.

D'Ariano, A., 2008. Improving real-time train dispatching: models, algorithms and applications, Department of Transport \& Planning. Delft University of Technology, Netherlands.

D’Ariano, A., Corman, F., Pacciarelli, D., Pranzo, M., 2008a. Reordering and local rerouting strategies to manage train traffic in real time. Transportation Science 42, 405-419.

D'Ariano, A., Pacciarelli, D., Pranzo, M., 2007a. A branch and bound algorithm for scheduling trains in a railway network. European Journal of Operational Research $183,643-657$.

D’Ariano, A., Pacciarelli, D., Pranzo, M., 2008b. Assessment of flexible timetables in real-time traffic management of a railway bottleneck. Transportation Research Part C: Emerging Technologies 16, 232-245.

D’Ariano, A., Pranzo, M., Hansen, I.A., 2007b. Conflict resolution and train speed coordination for solving real-time timetable perturbations. IEEE Transactions on Intelligent Transportation Systems - TITS 8, 208-222.

Daamen, W., Goverde, R.M.P., Hansen, I.A., 2008. Non-Discriminatory Automatic Registration of Knock-On Train Delays. Networks and Spatial Economics 9, 47-61.

Daamen, W., Goverde, R.M.P., Hansen, I.A., 2009. Non-Discriminatory Automatic Registration of Knock-On Train Delays. Networks and Spatial Economics 9, 47-61.

Davidsson, P., Henesey, L., Ramstedt, L., Törnquist, J., Wernstedt, F., 2005. An analysis of agent-based approaches to transport logistics. Transportation Research Part C: Emerging Technologies 13, 255-271.

Dessouky, M.M., Lu, Q., Zhao, J., Leachman, R.C., 2006. An Exact Solution Procedure for Determining the Optimal Dispatching.

Dorfman, M.J., Medanic, J., 2004. Scheduling trains on a railway network using a discrete event model of railway traffic. Transportation Research Part B: Methodological 38, 81-98. 
Elofson, G., Beranekb, P.M., Thomasc, P., 1997. An intelligent agent community approach to knowledge sharing. Decision Support Systems 20, 83-97.

ERA, UNISIG, EEIG, 2014. ERTMS/ETCS: SUBSET-026-3 System Requirements Specification, Chapter 3: Principles.

Fan, B., Roberts, C., Weston, P., 2011. A hybrid algorithm for optimal junction traffic control, in: Ricci, S., Hansen, I.A., Longo, G., Pacciarelli, D., Rodriguez, J., Wendler, E. (Eds.), 4th International Seminar on Railway Operations Modelling and Analysis (RailRome 2011), Rome.

Fay, A., 2000. A fuzzy knowledge-based system for railway traffic control. Engineering Applications of Artificial Intelligence 13, 719-729.

Freeman, E.H., 1990. A ${ }^{2}$ : An agent oriented programming architecture for multi-agent constraint satisfaction problems, 2nd International IEEE Conference on Tools for Artificial Intelligence. IEEE, Herndon, VA pp. 830-840.

Giannettoni, M., Savio, S., 2002. Traffic management in moving block railway systems: the results of the EU project COMBINE, in: Allen, J., Hill, R.J., Brebbia, C.A., Sciutto, G., Sone, S. (Eds.), Computers in Railways VIII. WIT Press, Lemnos, Greece, pp. 953-962.

Giannettoni, M., Savio, S., 2004. The European project COMBINE 2 to improve knowledge on future rail Traffic Management Systems, in: Brebbia, C.A., Allan, J., Hill, R.J., Sciutto, G., Sone, S. (Eds.), Computers in Railways IX. WIT Press, pp. 695-704.

Giuliari, M., Pellegrin, F., Savio, S., 2000. Moving block and traffic management in railway applications: the EU project COMBINE, in: Brebbia, C.A., Allen, J., Hill, R.J., Sciutto, G., Sone, S. (Eds.), Computers in Railways VII. WIT Press, Bologna, Italy, pp. 11-20.

Goverde, R.M.P., 2005. Punctuality of railway operations and timetable stability analysis Transport \& Planning Department. Delft University of Technology, The Netherlands.

Goverde, R.M.P., 2007. Railway timetable stability analysis using max-plus system theory. Transportation Research Part B: Methodological 41, 179-201.

Goverde, R.M.P., 2010. A delay propagation algorithm for large-scale railway traffic networks. Transportation Research Part C: Emerging Technologies 18, 269-287.

Goverde, R.M.P., Daamen, W., Hansen, I.A., 2008. Automatic identification of route conflicts occurrences and their consequences Computers in Railways XI. WIT Press, pp. 473-482. 
Goverde, R.M.P., Hansen, I.A., 2000. TNV-Prepare: Analysis of Dutch railway operations based on train detection data, in: Brebbia, C.A., Allan, J., Hill, R.J., Sciutto, G., Sone, S. (Eds.), Computers in Railways VII. WIT Press, Bologna, Italy, pp. 779-788.

Goverde, R.M.P., Meng, L., 2011. Advanced monitoring and management information of railway operations. Journal of Rail Transport Planning \& Management 1, 69-79.

Hansen, I.A., 2009. Railway network timetabling and dynamic traffic management, International Conference on Recent Advances in Railway Engineering, Tehran, Iran, pp. 135-145.

Hansen, I.A., Pachl, J., 2008. Railway Timetable and Traffic: Analysis, Modelling and Simulation. Eurailpress, Hamburg, Germany.

Higgins, A., Kozan, E., L., F., 1996. Optimal Scheduling of Trains on a Single Line Track. Transportation Research - Part B 30B, 147-161.

Ho, T.K., Yeung, T.H., 2000. Railway junction conflict resolution by genetic algorithm. Electronics Letters 36, 771-772.

Hooghiemstra, J.S., 1996. Design of regular interval timetables for strategic and tactical railway planning, in: Allan, J., Brebbia, C.A., Hill, R.J., Sciutto, G., Sone, S. (Eds.), Computers in Railways V, pp. 393-402.

Hooghiemstra, J.S., Kroon, L.G., Odijk, M.A., Salomon, M., Zwaneveld, P.J., 1999. Decision Support Systems Support the Search for Win-Win Solutions in Railway Network Design. Interfaces 29, 15-32.

Huisman, T., Boucherie, R.J., 2001. Running times on railway sections with heterogeneous train traffic. Transportation Research Part B: Methodological 35, 271-292.

Kim, K.-m., Hong, S.-h., Oh, S.-m., 2011. Development of a hierarchical conflict detection and resolution system, The 9th World Congress Railway Research, Challenge F: Even more trains even more on time, Lille, France.

Koning, J.A., 2002. Comparing the performance of ERTMS level 2 fixed block and ERTMS level 3 moving block signalling systems using simulation techniques, in: Allan, J., Hill, R.J., Brebbia, C.A., Sciutto, G., Sone, S. (Eds.), Computers in Railways VIII. witpress, Lemnos, Greece, pp. 43-52.

Lindner, T., 2000. Train Schedule Optimization in Public Rail Transport, Department of Mathematical Optimization. Braunschweig University of Technology, Braunschweig,Germany. 
Lu, F., Zhang, Z., 2009. Method for subway operation adjustment based on multi-agent, IEEE International Conference on Automation and Logistics Shenyang, China, pp. 1189-1193.

Lu, Q., Dessouky, M.M., Leachman, R.C., 2004. Modeling Train Movements through Complex Rail Networks. ACM Transactions on Modeling and Computer Simulation 14, 48-75.

Lusby, R., Larsen, J., Ehrgott, M., Ryan, D., 2011a. Railway track allocation: models and methods. OR Spectrum 33, 843-883.

Lusby, R., Larsen, J., Ehrgott, M., Ryan, D., 2013. A set packing inspired method for real-time junction train routing. Computers \& Operations Research 40, 713-724.

Lusby, R., Larsen, J., Ryan, D., Ehrgott, M., 2011b. Routing trains through railway junctions: a new set-packing approach. Transportation Science 45, 228-245

Macal, C.M., North, M.J., 2006. Tutorial on agent-based modeling and simulation Part 2: How to model with agents, in: Perrone, L.F., Wieland, F.P., Liu, J., Lawson, B.G., Nicol, D.M., Fujimoto, R.M. (Eds.), Proceedings of the 2006 Winter Simulation Conference. IEEE, Monterey, CA pp. 73-83.

Mascis, A., Pacciarelli, D., 2000. Machine scheduling via alternative graphs, Report DIA-46-2000, Roma, Italy.

Mascis, A., Pacciarelli, D., 2002. Job shop scheduling with blocking and no-wait constraints. European Journal of Operational Research 143, 498-517.

Mascis, A., Pacciarelli, D., Pranzo, M., 2002. Models and Algorithms for traffic management of rail networks, RT-DIA-74-2002.

Mascis, A., Pacciarelli, D., Stouwe, S.v.d., 2001. DSS for Real Time Traffic Optimisation, Proceedings of the 5th World Congress on Railway Research, Cologne, Germany, pp. 25-29.

Mazzarello, M., Copello, R., 2000. Conflict resolution in railway traffic control by a distributed intelligence approach, in: Brebbia, C.A., Allen, J., Hill, R.J., Sciutto, G., Sone, S. (Eds.), Computers in Railways VII. WIT Press, Bologna, Italy, pp. 789-798.

Mazzarello, M., Ottaviani, E., 2007. A traffic management system for real-time traffic optimisation in railways. Transportation Research Part B: Methodological 41, 246274.

Medeossi, G., Longo, G., de Fabris, S., 2011. A method for using stochastic blocking times to improve timetable planning. Journal of Rail Transport Planning \& Management 1, 1-13. 
Meester, L.E., Muns, S., 2007. Stochastic delay propagation in railway networks and phase-type distributions. Transportation Research Part B: Methodological 41, 218230 .

Mera, J.M., Gómez-Rey, I., Campos, A., 2008. ERTMS/ETCS test simulation bench, in: Brebbia, C.A. (Ed.), Urban Transport XIII: Urban Transport and the Environment in the 21st Century, pp. 259-271.

Mera, J.M., Gómez-Rey, I., Rodrigo, E., 2010. CBTC test simulation bench, in: Ning, B., Brebbia, C.A., Tomii, N. (Eds.), Computers in Railways XII, Beijing , China, pp. 485-495.

Mera, J.M., Gutiérrez, L.M., G6mez-Rey, A., Tapia, S., Arribas, A., Vera, C., Cabanellas, J.M., Romero, G., Maroto, J., Felez, J., 2002. Simulation of the ERTMS / ETCS Railways Control and Protection System; Levels 0, 1 and 2., in: Allan, J., Hill, R.J., Brebbia, C.A., Sciutto, G., Sone, S. (Eds.), Computers in Railways VIII. witpress, Lemnos, Greece, pp. 1119-1128.

Min, Y.-H., Park, M.-J., Hong, S.-P., Hong, S.-H., 2011. An appraisal of a columngeneration-based algorithm for centralized train-conflict resolution on a metropolitan railway network. Transportation Research Part B: Methodological 45, 409-429.

Murali, P., Dessouky, M., Ordóñez, F., Palmer, K., 2010. A delay estimation technique for single and double-track railroads. Transportation Research Part E: Logistics and Transportation Review 46, 483-495.

Narayanaswami, S., Rangaraj, N., 2015. A MAS architecture for dynamic, realtime rescheduling and learning applied to railway transportation. Expert Systems with Applications 42, 2638-2656.

Ouelhadj, D., Petrovic, S., 2009. A survey of dynamic scheduling in manufacturing systems. Journal of Scheduling 12, 417-431.

Pacciarelli, D., 2002. Alternative graph formulation for solving complex factoryscheduling problems International Journal of Production Research 40, 3641-3653.

Pacciarelli, D., Pranzo, M., 2001. A tabu search algorithm for the railway scheduling problem, MIC'2001 - 4th Metaheuristics International Conference, Porto, Portugal, pp. 159-163.

Park, S., Sugumaran, V., 2005. Designing multi-agent systems: a framework and application. Expert Systems with Applications 28, 259-271.

Pearson, L.V., 1973. Moving block railway signalling, Electronic and Electrical Engineering Loughborough University. 
Pentimelli, C., Marconi, L.F.G., 2001. Railway Transport Final Area Report, 4th Framework for RTD\&D, Transport Sector-TAP, pp. 21-23.

Proença, H., Oliveira, E., 2004. MARCS Multi-agent Railway Control System, 9th Ibero-American Conference on AI, Puebla, Mexico, pp. 12-21.

Samà, M., D'Ariano, A., Corman, F., Pacciarelli, D., 2016a. A variable neighbourhood search for fast train scheduling and routing during disturbed railway traffic situations. Computers \& Operations Research.

Samà, M., Pellegrini, P., D’Ariano, A., Rodriguez, J., Pacciarelli, D., 2016b. Ant colony optimization for the real-time train routing selection problem. Transportation Research Part B: Methodological 85, 89-108.

Schaafsma, A., Bartholomeus, M., 2007. Dynamic traffic management in the Schiphol bottleneck, in: Hansen, I.A., Radtke, A., Pachl, J., Wendler, E. (Eds.), CD-ROM Proceedings of the Second International Seminar on Railway Operations Modelling and Analysis, Hannover, Germany.

Schaefer, H., Pferdmenges, S., 1994. An expert system for real-time train dispatching, Computers in railways IV, Madrid, Spain, pp. 27-34.

Szpigel, B., 1973. Optimal train scheduling on a single track railway. Operational Research 72, 343-352.

Törnquist, J., 2005. Computer-based decision support for railway traffic scheduling and dispatching: A review of models and algorithms, 5th Workshop on Algorithmic Methods and Models for Optimization of Railways, ATMOS 2005, Palma de Mallorca, Spain.

Törnquist, J., Davidsson, P., 2002. A multi-agent system approach to train delay handling, ECAI-02 Workshop on Agent Technologies in Logistics, Lyon, France, pp. 50-53.

Törnquist, J., Persson, J.A., 2005. Train traffic deviation handling using Tabu Search and Simulated Annealing, IEEE Proceedings of the 38th Hawaii International Conference on System Sciences - 2005. IEEE.

Törnquist, J., Persson, J.A., 2007. N-tracked railway traffic re-scheduling during disturbances. Transportation Research Part B: Methodological 41, 342-362.

UNISIG, 2012. ERTMS/ETCS: SUBSET-041 Performance Requirements for Interoperability.

Wang, P., Ma, L., Goverde, R.P., Wang, Q., 2016. Rescheduling Trains Using Petri Nets and Heuristic Search. IEEE Transactions on Intelligent Transportation Systems - TITS 17, 726-736. 
Wen, C., 2010. Prediction of Train Operation Conflict, Proceedings of the 29th Chinese Control Conference, Beijing,China, pp. 5441-5446.

Yeung, T.H., Ho, T.K., 2000. Railway junction conflict resolution by local search method, in: Brebbia, C.A., Allen, J., Hill, R.J., Sciutto, G., Sone, S. (Eds.), Computers in Railways VII. WIT Press, Bologna, Italy, pp. 769-778.

Yuan, J., Hansen, I.A., 2007. Optimizing capacity utilization of stations by estimating knock-on train delays. Transportation Research Part B: Methodological 41, 202-217.

Zwaneveld, P.J., Kroon, L.G., Romeijn, H.E., Salomon, M., Dauzère-Pérès, S., van Hoesel, S.P.M., Ambergen, H.W., 1996. Routing trains through railway stations: model formulation and algorithms. Transportation Science 30, 181-194.

Zwaneveld, P.J., Kroon, L.G., van Hoesel, S.P.M., 2001. Routing trains through a railway station based on a node packing model. European Journal of Operational Research 128, 14-33. 



\section{About the author}

Taomei Zhu was born on March 15, 1986 in Wuyi, Zhejiang province, China. She received the B.S. in automation engineering from Southwest Jiaotong University, Chengdu, China, in 2008 and the M.S. degree in traffic information engineering and control from Beijing Jiaotong University, Beijing, China, in 2011.

In September 2011, she was sponsored by Chinese Scholarship Council (CSC) for further study at ETS Industrial Engineering, Polytechnic University of Madrid, Spain. Just then, she joined the Railway Technology Research Centre (CITEF). In 2013, she started her Ph.D. project under the supervision of Prof. Dr. José Manuel Mera Sánchez de Pedro. During her Ph.D. studies, she mainly worked on optimisation algorithms for railway traffic management.

Her research interests include railway traffic modelling and simulation, data analysis, railway operations research, intelligent traffic control and decision support systems. 